\title{
On locally analytic vectors of the completed cohomology of modular curves
}

Lue Pan

Department of Mathematics, Princeton University, Fine Hall, Washington Road, Princeton, NJ 08544-1000, USA;

E-mail: 1pan@princeton.edu.

Received: 7 September 2020; Revised: 29 November 2021; Accepted: 24 December 2021

2020 Mathematics Subject Classification: 11F77

\begin{abstract}
We study the locally analytic vectors in the completed cohomology of modular curves and determine the eigenvectors of a rational Borel subalgebra of $\mathfrak{g l}_{2}\left(\mathbb{Q}_{p}\right)$. As applications, we prove a classicality result for overconvergent eigenforms of weight 1 and give a new proof of the Fontaine-Mazur conjecture in the irregular case under some mild hypotheses. For an overconvergent eigenform of weight $k$, we show its corresponding Galois representation has Hodge-Tate-Sen weights $0, k-1$ and prove a converse result.
\end{abstract}

\section{Contents}

1 Introduction

2 Locally analytic vectors $\quad 9$

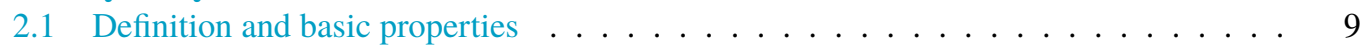

2.2 Derived functor . . . . . . . . . . . . . . . . . 11

$3 \quad$ Locally analytic vectors and relative Sen theory $\quad 13$

3.1 Statement of the main result . . . . . . . . . . . . . . . . . 13

3.2 Relative Sen theory . . . . . . . . . . . . . . . . . . 15

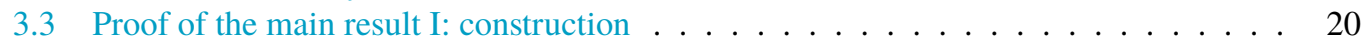

3.4 Proof of the main result II: uniqueness . . . . . . . . . . . . . . . . . 23

3.5 Locally analytic covering . . . . . . . . . . . . . . . . . 25

3.6 Application: acyclicity of taking locally analytic vectors of $B \ldots \ldots$. . . . . . . 27

4 Locally analytic functions on perfectoid modular curves $\quad 30$

4.1 Modular curves and the Hodge-Tate period map . . . . . . . . . . . . . . . . . . 30

4.2 Faltings's extension and computation of $\theta \ldots \ldots \ldots$

4.3 Local structure of $\mathcal{O}_{K}^{\text {la }} \ldots \ldots \ldots \ldots \ldots \ldots \ldots \ldots$

4.4 Cohomology of $\mathcal{O}_{K}^{\text {la }}$ and completed cohomology . . . . . . . . . . . . 43

$5 \mu$-isotypic part of completed cohomology 44

5.1 A $p$-adic Hodge-theoretic interpretation of $\theta_{\mathfrak{h}} \ldots \ldots \ldots \ldots$

$5.2 \Uparrow$-cohomology $(\mathrm{I}) \ldots \ldots \ldots \ldots \ldots \ldots$

$5.3 \pi$-cohomology $(\mathrm{II}) \ldots \ldots \ldots \ldots \ldots \ldots \ldots$

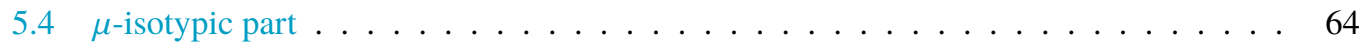

$\begin{array}{lll}6 & \text { Applications } & 67\end{array}$

6.1 Hecke algebra ................................ 67

(C) The Author(s), 2022. Published by Cambridge University Press. This is an Open Access article, distributed under the terms of the Creative Commons Attribution licence (https://creativecommons.org/licenses/by/4.0/), which permits unrestricted re-use, distribution, and reproduction in any medium, provided the original work is properly cited. 
6.2 A classicality result for overconvergent weight 1 forms . . . . . . . . . . . . . . . 69

6.3 Local-global compatibility . . . . . . . . . . . . . . . . . 71

6.4 Colmez's Kirillov model . . . . . . . . . . . . . . . . . . . . 75

$\begin{array}{ll}\text { Acknowledgements } & 79\end{array}$

$\begin{array}{lr}\text { Financial Support } & 79\end{array}$

$\begin{array}{lr}\text { References } & 79\end{array}$

\section{Introduction}

Let $p$ be a rational prime. In his pioneering work [Eme06b], Emerton introduced completed cohomology, which $p$-adically interpolates automorphic representations, and explained how techniques of locally analytic $p$-adic representation theory may be applied to study it. In this article, we will focus on the simplest (non-abelian) case: completed cohomology of the modular curves. More precisely, let $K$ be an open subgroup $\mathrm{GL}_{2}\left(\mathbb{A}_{f}\right)$, where $\mathbb{A}_{f}$ denotes the ring of finite adèles of $\mathbb{Q}$. We have the modular curve of level $K$

$$
Y_{K}(\mathbb{C})=\mathrm{GL}_{2}(\mathbb{Q}) \backslash\left(\mathbb{H}^{ \pm 1} \times \mathrm{GL}_{2}\left(\mathbb{A}_{f}\right) / K\right)
$$

where $\mathbb{H}^{ \pm 1}=\mathbb{C}-\mathbb{R}$ is the union of the usual upper and lower half-planes. Denote by $\mathbb{A}_{f}^{p}$ the prime-to- $p$ part of $\mathbb{A}_{f}$. For an open subgroup $K^{p}$ of $\mathrm{GL}_{2}\left(\mathbb{A}_{f}^{p}\right)$, the completed cohomology of tame level $K^{p}$ is defined as

$$
\tilde{H}^{i}\left(K^{p}, \mathbb{Z}_{p}\right):=\lim _{n} \underset{K_{p} \subset \mathrm{GL}_{2}\left(\mathbb{Q}_{p}\right)}{\lim } H^{i}\left(Y_{K^{p}} K_{p}(\mathbb{C}), \mathbb{Z} / p^{n}\right)
$$

It is $p$-adically complete and equipped with a natural continuous action of $\mathrm{GL}_{2}\left(\mathbb{Q}_{p}\right)$. Our main tool to study this will be ( $p$-adic) Hodge theory. Hence, we would like to extend the coefficients $\mathbb{Q}_{p}$ to a complete, algebraically closed field. So let $C=\mathbb{C}_{p}$, the completion of an algebraic closure $\overline{\mathbb{Q}_{p}}$ of $\mathbb{Q}_{p}$, and $\mathcal{O}_{C}$ be its ring of integers. Consider

$$
\tilde{H}^{i}\left(K^{p}, \mathcal{O}_{C}\right):={\underset{\lim }{n}}_{K_{p} \subset \mathrm{GL}_{2}\left(\mathbb{Q}_{p}\right)} H^{i}\left(Y_{K^{p} K_{p}}(\mathbb{C}), \mathcal{O}_{C} / p^{n}\right) .
$$

Then $\tilde{H}^{i}\left(K^{p}, C\right):=\tilde{H}^{i}\left(K^{p}, \mathcal{O}_{C}\right) \otimes_{\mathcal{O}_{C}} C$ is a $\mathbb{Q}_{p}$-Banach space representation of $\mathrm{GL}_{2}\left(\mathbb{Q}_{p}\right)$. As in [ST03], we can consider its locally analytic vectors $\tilde{H}^{i}\left(K^{p}, C\right)^{\text {la }}$. Let

$\circ \mathfrak{g}=C \otimes_{\mathbb{Q}_{p}} \mathfrak{g l}_{2}\left(\mathbb{Q}_{p}\right)$ : the 'complexified' Lie algebra of $\mathrm{GL}_{2}\left(\mathbb{Q}_{p}\right)$;

○ $B \subset \mathrm{GL}_{2}\left(\mathbb{Q}_{p}\right)$ : the upper-triangular Borel subgroup;

$\circ \mathfrak{b} \subset \mathfrak{g}$ : the 'complexified' Lie algebra of $B$.

The Lie algebra $\mathfrak{g}$ (in particular $\mathfrak{b})$ acts naturally on $\tilde{H}^{i}\left(K^{p}, C\right)^{\text {la }}$ by the infinitesimal action of $\mathrm{GL}_{2}\left(\mathbb{Q}_{p}\right)$. Given a character $\mu: \mathfrak{b} \rightarrow C$, one main goal of this article is to study the $\mu$-isotypic part $\tilde{H}^{i}\left(K^{p}, C\right)_{\mu}^{\text {la }}$. We can pass to the direct limit over all tame levels $K^{p}$ :

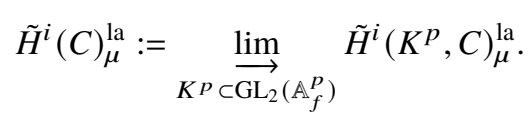

Clearly, there is a natural action of $\mathrm{GL}_{2}\left(\mathbb{A}_{f}^{p}\right) \times B$ on it.

To state our result, we need more notation. The open modular curve $Y_{K}(\mathbb{C})$ has a natural compactification $X_{K}(\mathbb{C})$ by adding cusps. This complete curve $X_{K}(\mathbb{C})$ has a natural model $X_{K}$ over $\mathbb{Q}$, and we denote by $X_{K, C}$ its base change to $C$. Let $k$ be an integer. We have the usual automorphic line bundle 
$\omega^{k}$ on $X_{K, C}$ whose global sections correspond to level- $K$ modular forms of weight $k$ (with coefficients in $C$ ). Denote by

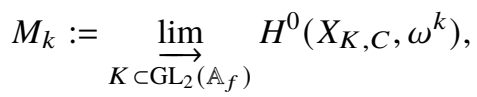

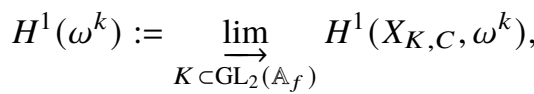

where both limits are taken over all open compact subgroups of $\mathrm{GL}_{2}\left(\mathbb{A}_{f}\right)$. Both spaces have natural actions of $\mathrm{GL}_{2}\left(\mathbb{A}_{f}\right)$ and relate to automorphic representations of $\mathrm{GL}_{2}(\mathbb{A})$ (after choosing an isomorphism $C \cong \mathbb{C}$ ). Here $\mathbb{A}$ denotes the ring of adèles of $\mathbb{Q}$.

We also need players which are special in this $p$-adic story: overconvergent modular forms. This will be slightly different from the usual one in the literature as we want an action of the Borel $B$. We denote by $\mathcal{X}_{K}$ the rigid analytic space associated to $X_{K, C}$. Let $\Gamma\left(p^{n}\right)=1+p^{n} M_{2}\left(\mathbb{Z}_{p}\right) \subset \mathrm{GL}_{2}\left(\mathbb{Z}_{p}\right)$ be the principal congruence subgroup of level $n \geq 2$ and let $K^{p}$ be a tame level. As explained by Katz-Mazur [KM85, Theorem 13.7.6], $X_{K^{p}} \Gamma\left(p^{n}\right), C$ has a natural integral model over $\mathcal{O}_{C}$, and the irreducible components of its special fibre can be indexed by surjective homomorphisms $\left(\mathbb{Z} / p^{n}\right)^{2} \rightarrow \mathbb{Z} / p^{n}$. We denote by $\mathcal{X}_{K^{p} \Gamma\left(p^{n}\right), c} \subset \mathcal{X}_{K^{p} \Gamma\left(p^{n}\right)}$ the tubular neighbourhood of the non-supersingular points of irreducible components of indices sending $(1,0) \in\left(\mathbb{Z} / p^{n}\right)^{2}$ to 0 . Let $M_{k}^{\dagger}\left(K^{p} \Gamma\left(p^{n}\right)\right)$ be the space of sections of $\omega^{k}$ defined on a strict neighbourhood of $\mathcal{X}_{K} \Gamma_{\Gamma}\left(p^{n}\right), c$. Overconvergent modular forms of weight $k$ are defined as

$$
M_{k}^{\dagger}:=\underset{K^{p} \subset \mathrm{GL}_{2}\left(\mathbb{A}_{f}^{p}\right)}{\stackrel{\lim }{\longrightarrow}} M_{k}^{\dagger}\left(K^{p} \Gamma\left(p^{n}\right)\right) .
$$

One can check that there is a natural action of $\mathrm{GL}_{2}\left(\mathbb{A}_{f}^{p}\right) \times B$ on it.

Since modular curves can be defined over $\mathbb{Q}_{p}$, the absolute Galois group $G_{\mathbb{Q}_{p}}=\operatorname{Gal}\left(\overline{\mathbb{Q}_{p}} / \mathbb{Q}_{p}\right)$ acts on $C$ and everything we have defined and commutes with the action of $\mathrm{GL}_{2}\left(\mathbb{A}_{f}^{p}\right) \times B$. Our main results describe $\tilde{H}^{i}(C)_{\mu}^{\text {la }}$ as a representation of $\mathrm{GL}_{2}\left(\mathbb{A}_{f}^{p}\right) \times B \times G_{\mathbb{Q}_{p}}$.

We need the following characters of $\mathrm{GL}_{2}\left(\mathbb{A}_{f}^{p}\right) \times B$ for stating our result. Denote by $\varepsilon: \mathbb{A}_{f}^{\times} / \mathbb{Q}_{>0}^{\times} \rightarrow \mathbb{Z}_{p}^{\times}$ the $p$-adic cyclotomic character (via class field theory, which sends a uniformiser $l \neq p$ to a geometric Frobenius). We define $t: \mathrm{GL}_{2}\left(\mathbb{A}_{f}\right) \rightarrow \mathbb{Z}_{p}^{\times}$as $\varepsilon \circ$ det and view it as a character of $\mathrm{GL}_{2}\left(\mathbb{A}_{f}^{p}\right) \times B$ by restriction. For $i=1,2$, we define $e_{i}: B \rightarrow \mathbb{Q}_{p}^{\times}$by sending $\left(\begin{array}{cc}a_{1} & b \\ 0 & a_{2}\end{array}\right) \in B$ to $a_{i}$ and view them as characters of $\mathrm{GL}_{2}\left(\mathbb{A}_{f}^{p}\right) \times B$ by projecting onto $B$. We write $\cdot$ rather than $\otimes$ when twisting by such a character. One main result of this article is as follows. (This is obtained from Theorem 5.4.2 by taking direct limit over all $K^{p}$. There is a small difference as we do not put a Galois action on $e_{1}, e_{2}, t$ here.)

Theorem 1.0.1. Let $k \in \mathbb{Z}$ and let $\mu=\mu_{k}: \mathfrak{b} \rightarrow C$ be the character sending $\left(\begin{array}{ll}a & b \\ 0 & d\end{array}\right)$ to $k d$. Assume $k \neq 1$. There is a natural decomposition

$$
\tilde{H}^{1}(C)_{\mu_{k}}^{\mathrm{la}}=N_{k, 1} \cdot e_{1}^{-k} t^{k} \oplus N_{k, w}(k-1) \cdot e_{1}^{-1} e_{2}^{k-1} t,
$$

where $(k-1)$ denotes a Tate twist by $k-1$. Moreover, we have the following description of $N_{k, 1}$ and $N_{k, w}$ :

(1) $N_{k, w} \cong M_{2-k}^{\dagger}$ for $k \neq 2$. When $k=2$, we have

$$
0 \rightarrow M_{0}^{\dagger} / M_{0} \rightarrow N_{k, w} \rightarrow M_{0} \rightarrow 0
$$


(2) If $k \leq-1$, then there is an exact sequence

$$
0 \rightarrow H^{1}\left(\omega^{k}\right) \rightarrow N_{k, 1} \rightarrow M_{k}^{\dagger} \rightarrow 0
$$

(3) If $k=0$, then there is an exact sequence

$$
0 \rightarrow H^{1}\left(\omega^{0}\right) \rightarrow N_{k, 1} \rightarrow M_{0}^{\dagger} / M_{0} \rightarrow 0
$$

(4) If $k \geq 2$, then $N_{k, 1} \cong M_{k}^{\dagger} / M_{k}$.

All of the maps and isomorphisms here are $\mathrm{GL}_{2}\left(\mathbb{A}_{f}^{p}\right) \times B \times G_{\mathbb{Q}_{p}}$-equivariant.

One may view this as a Hodge-Tate decomposition into weight 0 and $1-k$ components. Hence, this is a $p$-adic analogue of the Shimura isomorphism. Our convention is that the cyclotomic character is of Hodge-Tate weight -1 . We have a similar (Hodge-Tate-Sen) decomposition if $k \notin \mathbb{Z}$ (Theorem 5.4.2). Unfortunately, we can only determine half of the decomposition, which roughly is given by overconvergent modular forms of weight $2-k$. We will give a definition of this space for nonintegral weights in Definition 5.2.3.

When $k=1$, we do not get a decomposition because of the existence of non-Hodge-Tate representations with equal Hodge-Tate-Sen weights. Here is our result (which follows from Theorem 5.4.6 by taking the direct limit over all $K^{p}$ ).

Theorem 1.0.2. Suppose $\mu=\mu_{1}: \mathfrak{b} \rightarrow C$ sends $\left(\begin{array}{ll}a & b \\ 0 & d\end{array}\right)$ to $d$. There is a natural exact sequence

$$
0 \rightarrow N_{1} \cdot e_{1}^{-1} t \rightarrow \tilde{H}^{1}(C)_{\mu_{1}}^{\mathrm{la}} \rightarrow M_{1}^{\dagger} \cdot e_{1}^{-1} t \rightarrow 0
$$

where $N_{1}$ sits inside an exact sequence:

$$
0 \rightarrow M_{1}^{\dagger} / M_{1} \rightarrow N_{1} \rightarrow H^{1}(\omega) \rightarrow 0 .
$$

Moreover, let $\tilde{H}^{1}(C)_{\mu_{1}}^{\mathrm{la},(1,0)} \subset \tilde{H}^{1}(C)_{\mu_{1}}^{\mathrm{la}}$ be the pullback of $M_{1} \cdot e_{1}^{-1} t \subset M_{1}^{\dagger} \cdot e_{1}^{-1} t$, so that there is an exact sequence

$$
0 \rightarrow N_{1} \cdot e_{1}^{-1} t \rightarrow \tilde{H}^{1}(C)_{\mu_{1}}^{\mathrm{la},(1,0)} \rightarrow M_{1} \cdot e_{1}^{-1} t \rightarrow 0
$$

Then $\left(\tilde{H}^{1}(C)_{\mu_{1}}^{\mathrm{la}}\right)^{G_{Q_{p}}} \subset \tilde{H}^{1}(C)_{\mu_{1}}^{\mathrm{la},(1,0)}$. Again, all of the maps here are $\mathrm{GL}_{2}\left(\mathbb{A}_{f}^{p}\right) \times B \times G_{\mathbb{Q}_{p}}$-equivariant.

In fact, we will generalise the usual Sen operator to this situation in Definition 5.1.5 and then show it is nilpotent on $\tilde{H}^{1}(C)_{\mu}^{\mathrm{la}}$ and factors through the quotient $M_{1}^{\dagger} \cdot e_{1}^{-1} t$ and maps it to $M_{1}^{\dagger} / M_{1} \cdot e_{1}^{-1} t \subset N_{1} \cdot e_{1}^{-1} t$.

Both theorems give a concrete relation between completed cohomology and overconvergent modular forms. As a first application, we are able to prove a classicality result for weight 1 overconvergent eigenforms. Fix a tame level $K^{p}=K^{S} K_{S \backslash\{p\}}$ for some finite set of primes $S$ containing $p$ and suppose $K^{S}$ is a maximal open compact subgroup of $\mathrm{GL}_{2}\left(\mathbb{A}_{f}^{S}\right)$, where $\mathbb{A}_{f}^{S}$ is the prime-to- $S$ part of $\mathbb{A}_{f}$. Denote by $\mathbb{T}_{K^{p}}:=\mathbb{Z}_{p}\left[\mathrm{GL}_{2}\left(\mathbb{A}_{f}^{S}\right) / / K^{S}\right]$ the abstract Hecke algebra of $K^{S}$-bi-invariant compactly supported functions on $\mathrm{GL}_{2}\left(\mathbb{A}_{f}^{S}\right)$, where the Haar measure gives $K^{S}$ measure 1 . Then $\mathbb{T}_{K^{p}}$ acts on $H^{1}\left(Y_{K^{p}} K_{p}(\mathbb{C}), \mathbb{Z}_{p}\right)$ and we denote its image in $\operatorname{End}_{\mathbb{Z}_{p}}\left(H^{1}\left(Y_{K^{p} K_{p}}(\mathbb{C}), \mathbb{Z}_{p}\right)\right)$ by $\mathbb{T}\left(K^{p} K_{p}\right)$ and let $\mathbb{T}:=\lim _{K_{p}} \mathbb{T}\left(K^{p} K_{p}\right)$. It can be shown that $\mathbb{T}$ is independent of $S$. Let $\lambda: \mathbb{T} \rightarrow \overline{\mathbb{Q}_{p}}$ be a $\mathbb{Z}_{p}$-algebra homomorphism. We can associate to $\lambda$ a 2-dimensional semi-simple Galois representation

$$
\rho_{\lambda}: \operatorname{Gal}(\overline{\mathbb{Q}} / \mathbb{Q}) \rightarrow \mathrm{GL}_{2}\left(\overline{\mathbb{Q}}_{p}\right)
$$


satisfying $\operatorname{tr}\left(\rho_{\lambda}\left(\operatorname{Frob}_{l}\right)\right)=\lambda\left(T_{l}\right) l^{-1}$ for $l \notin S$. Here $T_{l}=\left[K^{S}\left(\begin{array}{ll}l & 0 \\ 0 & 1\end{array}\right) K^{S}\right]$ and Frob $_{l}$ is a lift of geometric Frobenius at $l$. See Section 6.1.1 for more details.

Let $M_{1}^{\dagger}\left(K^{p}\right)=\lim _{\longrightarrow} M_{1}^{\dagger}\left(K^{p} \Gamma\left(p^{n}\right)\right)$ and $N_{0}=\left(\begin{array}{cc}1 & \mathbb{Z}_{p} \\ 0 & 1\end{array}\right) \subset \mathrm{GL}_{2}\left(\mathbb{Q}_{p}\right)$. We have the usual $U_{p}$-operator acting on $N_{0}$-invariants by

$$
U_{p}:=\sum_{i=0}^{p-1}\left(\begin{array}{ll}
p & i \\
0 & 1
\end{array}\right) .
$$

Since $\mathbb{T}$ acts on $\tilde{H}^{1}\left(K^{p}, \mathbb{Z}_{p}\right)$, it follows from Theorem 1.0.2 that $\mathbb{T}$ acts on $M_{1}^{\dagger}\left(K^{p}\right)$. We denote by $\mathfrak{p}_{\lambda}$ the kernel of $\lambda$ and by $M_{1}^{\dagger}\left(K^{p}\right)\left[\mathfrak{p}_{\lambda}\right]$ the $\lambda$-isotypic part of $M_{1}^{\dagger}\left(K^{p}\right)$.

Theorem 1.0.3 (Theorem 6.2.2). Suppose

○ $M_{1}^{\dagger}\left(K^{p}\right)\left[\mathfrak{p}_{\lambda}\right]^{N_{0}} \neq 0$ has a nonzero $U_{p}$-eigenvector;

- $\left.\rho_{\lambda}\right|_{G_{Q_{p}}}$ is Hodge-Tate of weights 0,0 .

Then $\lambda$ comes from a classical weight 1 eigenform; that is, $M_{1}\left(K^{p}\right)\left[\mathfrak{p}_{\lambda}\right] \neq 0$.

In the ordinary case, this reproves a result of Buzzard-Taylor [BT99]. Note that we do not assume the $U_{p}$-eigenvalue is nonzero. This will allow us to attack the Fontaine-Mazur conjecture in the nonordinary, irregular case.

Proof. We give a sketch of the proof here. We may assume $\rho_{\lambda}$ is irreducible. Let $\mu_{1}$ be as in Theorem 1.0.2 and denote by $\lambda \cdot t: \mathbb{T} \rightarrow \overline{\mathbb{Q}}_{p}$ the map which sends $T_{l}$ to $\lambda\left(T_{l}\right) l^{-1}$. The key point is that by the Eichler-Shimura relation, $\tilde{H}^{1}\left(K^{p}, C\right)_{\mu_{1}}^{\text {la }}\left[\mathfrak{p}_{\lambda \cdot t}\right]=\rho_{\lambda} \otimes_{\overline{\mathbb{Q}}_{p}} W$ for some $W$. Since $\rho_{\lambda}$ is Hodge-Tate of weights 0,0 , we have $\tilde{H}^{1}\left(K^{p}, C\right)_{\mu_{1}}^{\mathrm{la}}\left[\mathfrak{p}_{\lambda \cdot t}\right]=\tilde{H}^{1}(C)_{\mu_{1}}^{\mathrm{la},(1,0)}\left[\mathfrak{p}_{\lambda \cdot t}\right]$. Now assume $\lambda$ is not classical. Then it follows from the second part of Theorem 1.0.2 that

$$
\left(M_{1}^{\dagger}\left(K^{p}\right)\left[\mathfrak{p}_{\lambda}\right] \cdot e_{1}^{-1} t\right)^{N_{0}}=\tilde{H}^{1}(C)_{\mu_{1}}^{\mathrm{la},(1,0)}\left[\mathfrak{p}_{\lambda \cdot t}\right]^{N_{0}}=\tilde{H}^{1}\left(K^{p}, C\right)_{\mu_{1}}^{\mathrm{la}}\left[\mathfrak{p}_{\lambda \cdot t}\right]^{N_{0}}=\rho_{\lambda} \otimes W^{N_{0}} .
$$

By choosing suitable $K^{p}$ and taking suitable eigenvectors of Hecke operators $U_{l}$ and diamond operators at ramified places, the nonzero $U_{p}$-eigenspace of the first term will be 1 -dimensional by the $q$-expansion principle. However, the corresponding subspace of the last term will always have dimension at least $\operatorname{dim} \rho_{\lambda}=2$ if it is nonzero! Contradiction. This shows that $\lambda$ has to be classical.

Thanks to Emerton's work on local-global compatibility, we have a good understanding of $\tilde{H}^{1}\left(K^{p}, C\right)\left[\mathfrak{p}_{\lambda}\right]$ in terms of the $p$-adic local Langlands correspondence. Colmez's Kirillov model then shows that a $U_{p}$-eigenvector always exists (see Corollary 6.4.6).

Theorem 1.0.4 (Theorem 6.4.7). Suppose

- $\rho_{\lambda}$ is irreducible;

- $\left.\rho_{\lambda}\right|_{G_{Q_{p}}}$ is Hodge-Tate of weight 0,0 .

Then $\lambda$ is classical; that is, $M_{1}\left(K^{p}\right)\left[\mathfrak{p}_{\lambda}\right] \neq 0$ for some tame level $K^{p}$.

We say a Galois representation $\rho$ is pro-modular if $\rho=\rho_{\lambda}$ for some $K^{p}$ and $\lambda$. Combining Theorem 1.0.4 with work on promodularity of a Galois representation ([Eme11, Theorem 1.2.3], which is based on previous work of Böckle, Diamond-Flach-Guo, Khare-Wintenberger and Kisin), we give a new proof of the Fontaine-Mazur conjecture in the irregular case under some mild hypotheses.

Theorem 1.0.5 (Theorem 6.4.8 and Corollary 6.4.9). Let $p>2$ be a prime number and $\rho: \operatorname{Gal}(\overline{\mathbb{Q}} / \mathbb{Q}) \rightarrow$ $\mathrm{GL}_{2}\left(\overline{\mathbb{Q}}_{p}\right)$ a continuous irreducible 2-dimensional representation. Assume 
(1) $\rho$ is unramified outside of finitely many primes;

(2) $\operatorname{det} \rho(c)=-1$ for a complex conjugation $c \in \mathrm{Gal}(\overline{\mathbb{Q}} / \mathbb{Q})$;

(3) $\left.\rho\right|_{G_{Q_{p}}}$ is Hodge-Tate of weights 0,0 ;

(4) the residual representation $\left.\bar{\rho}\right|_{\mathrm{Gal}\left(\overline{\mathbb{Q}} / \mathbb{Q}\left(\mu_{p}\right)\right)}$ is irreducible;

(5) $\left(\left.\bar{\rho}\right|_{G_{Q_{p}}}\right)^{s s}$ is either irreducible or of the form $\eta_{1} \oplus \eta_{2}$ for some characters $\eta_{1}, \eta_{2}$ satisfying $\eta_{1} / \eta_{2} \neq 1, \omega^{ \pm 1}$.

Then $\rho$ is modular in the sense that it is isomorphic to the representation attached to a classical weight 1 cuspidal eigenform by [DS74]. In particular, $\rho$ has finite image.

Remark 1.0.6. This result was already known by the work of Pilloni-Stroh [PS16]. Before their work, much work on this problem was done in the ordinary case (Buzzard-Taylor [BT99], Calegari-Geraghty [CG18] and others) and has been generalised to the case of Hilbert modular forms. I hope this article gives a new perspective on this problem.

If the results of this article can also be generalised to Hilbert case, one should be able to remove the last two conditions by invoking our previous work on promodularity [Pan19].

For overconvergent eigenforms of weight not necessarily 1 , we have the following result.

Theorem 1.0.7 (Theorem 6.4.11). Suppose $\rho_{\lambda}$ is irreducible and $M_{k}^{\dagger}\left(K^{p}\right)\left[\mathfrak{p}_{\lambda}\right] \neq 0$; then $\left.\rho_{\lambda}\right|_{G_{Q_{p}}}$ has Hodge-Tate-Sen weights $0, k-1$. Conversely, suppose

- $\left.\rho_{\lambda}\right|_{G_{Q_{p}}}$ is irreducible and has Hodge-Tate-Sen weights $0, k-1$;

then $M_{k}^{\dagger}\left(K^{p}\right)\left[\mathfrak{p}_{\lambda}\right] \neq 0$.

Remark 1.0.8. As pointed out by a referee, Gouvêa in [Gou94, Conjecture 4] conjectured that the Hodge-Tate weights of the Galois representation associated to an overconvergent eigenform of weight $k$ are $0, k-1$ and guessed that this should be an equivalence. Our result confirms his conjecture and his guess under the given hypotheses.

We note that Sean Howe also proved Gouvêa's conjecture in [How21] independently. Recently, we found a more direct way to prove Gouvêa's conjecture in [Pan20] using Scholze's fake-Hasse invariants. But the method in [Pan20] does not seem to be enough to give a converse result. Indeed, one key ingredient in our proof of Theorem 1.0.7 here is Colmez's Kirillov model [Col10, Chap. VI], which is a deep result in $p$-adic local Langlands correspondence for $\mathrm{GL}_{2}\left(\mathbb{Q}_{p}\right)$.

Now we explain our strategy to compute $\tilde{H}^{1}(C)_{\mu}^{\mathrm{la}}$. As mentioned before, our main tool is $p$-adic Hodge theory. Fix a tame level $K^{p}$. By the work of Scholze [Sch15], there is an adic space $\mathcal{X}_{K^{p}} \sim \lim _{K_{p}} \mathcal{X}_{K^{p}} K_{p}$ (the modular curve at infinite level) with Hodge-Tate period map $\pi_{\mathrm{HT}}: \mathcal{X}_{K^{p}} \rightarrow \mathscr{F} \ell=\mathbb{P}^{1}$, where $\mathscr{F} \ell$ denotes the adic space associated to the flag variety of $\mathrm{GL}_{2} / C$. Let $\mathcal{O}_{K^{p}}$ be the pushforward of the structure sheaf of $\mathcal{X}_{K^{p}}$ along $\pi_{\mathrm{HT}}$. Then Scholze shows that there is a natural isomorphism

$$
\tilde{H}^{i}\left(K^{p}, C\right) \cong H^{i}\left(\mathscr{F} \ell, \mathcal{O}_{K^{p}}\right),
$$

where the right-hand side is computed on the analytic site of $\mathscr{F} \ell$. In order to study the $\left(\mathrm{GL}_{2}\left(\mathbb{Q}_{p}\right)\right.$-)locally analytic vectors, we consider the subsheaf of locally analytic sections $\mathcal{O}_{K^{p}}^{\text {la }} \subset \mathcal{O}_{K^{p}}$ (Section 4.2.6).

Theorem 1.0.9 (Theorem 4.4.6). There is a natural isomorphism

$$
\tilde{H}^{i}\left(K^{p}, C\right)^{\mathrm{la}} \cong H^{i}\left(\mathscr{F} \ell, \mathcal{O}_{K^{p}}^{\mathrm{la}}\right) .
$$

Next we need a careful local study of the sheaf $\mathcal{O}_{K^{p}}^{\text {la }}$. This part is largely inspired by the work of Berger-Colmez [BC16]. There are two key ingredients:

(1) $\mathcal{O}_{K^{p}}^{\text {la }}$ satisfies a differential equation on $\mathscr{F} \ell$;

(2) an explicit description of sections of $\mathcal{O}_{K^{p}}^{\text {la }}$ (Theorem 4.3.9). 
We will only explain the differential equation in detail here. It turns out to be nothing but the horizontal nilpotent subalgebra. To be more precise, we follow some constructions on the flag variety from [BB83]. Recall $\mathfrak{g}=\mathfrak{g l}_{2}(C)$. For a $C$-point $x$ of the flag variety $\mathrm{Fl}$ of $\mathrm{GL}_{2} / C$, let $\mathfrak{b}_{x}, \mathfrak{n}_{x} \subset \mathfrak{g}$ be its corresponding Borel subalgebra and nilpotent subalgebra. Let

$$
\begin{aligned}
\mathfrak{g}^{0} & :=\mathcal{O}_{\mathrm{Fl}} \otimes_{C} \mathfrak{g}, \\
\mathfrak{b}^{0} & :=\left\{f \in \mathfrak{g}^{0} \mid f_{x} \in \mathfrak{b}_{x}, \text { for all } x \in \operatorname{Fl}(C)\right\}, \\
\mathfrak{n}^{0} & :=\left\{f \in \mathfrak{g}^{0} \mid f_{x} \in \mathfrak{n}_{x}, \text { for all } x \in \operatorname{Fl}(C)\right\}
\end{aligned}
$$

By abuse of notation, we also view these as vector bundles on $\mathscr{F} \ell$. Then $\mathfrak{g}^{0}$ acts on $\mathcal{O}_{K^{p}}^{\text {la }}$ in an obvious way.

Theorem 1.0.10 (Theorem 4.2.7). $\mathfrak{n}^{0}$ acts trivially on $\mathcal{O}_{K^{p}}^{\text {la }}$.

In fact, we will show the existence of such a differential equation in a very general setting.

Theorem 1.0.11 (Theorem 3.1.2). Suppose

○ $X=\operatorname{Spa}\left(A, A^{+}\right):$a 1-dimensional smooth affinoid adic space (over $\operatorname{Spa}\left(C, \mathcal{O}_{C}\right)$ );

- $G$ : a finite-dimensional compact p-adic Lie group;

- $\operatorname{Spa}\left(B, B^{+}\right)$: a Galois pro-étale perfectoid covering of $X$ with Galois group $G$.

Then under some smallness assumption on $X$ (cf. Section 3.1.1), there is an element of $B \otimes_{\mathbb{Q}_{p}} \operatorname{Lie}(G)$ that annihilates the $G$-locally analytic vectors in $B$. It is nonzero if $\operatorname{Spa}\left(B, B^{+}\right)$is a locally analytic covering in the sense of Definition 3.5.4.

Remark 1.0.12. This can be viewed as a $p$-adic analogue of the Cauchy-Riemann equation in classical complex analysis. See also Remarks 3.1.4, 3.1.7 for the relation with classical and relative Sen theory (also called the $p$-adic Simpson correspondence in the literature). We can also allow some ramification; cf. Theorem 3.1.2.

Example 1.0.13. One simple example is

○ $X=\operatorname{Spa}\left(C\left\langle T^{ \pm 1}\right\rangle, \mathcal{O}_{C}\left\langle T^{ \pm 1}\right\rangle\right)$, the 1-dimensional torus;

○ $\widetilde{X}=\operatorname{Spa}\left(C\left\langle T^{ \pm \frac{1}{p^{\infty}}}\right\rangle, \mathcal{O}_{C}\left\langle T^{ \pm \frac{1}{p^{\infty}}}\right\rangle\right)$, the perfectoid inverse limit of $\operatorname{Spa}\left(C\left\langle T^{ \pm \frac{1}{p^{m}}}\right\rangle, \mathcal{O}_{C}\left\langle T^{ \pm} \frac{1}{p^{m}}\right\rangle\right)$;

$\circ G \cong \mathbb{Z}_{p}$.

In this case, the $G$-locally analytic vectors in $C\left\langle T^{ \pm \frac{1}{p^{\infty}}}\right\rangle$ are the smooth vectors $\bigcup_{m \in \mathbb{N}} C\left\langle T^{ \pm \frac{1}{p^{m}}}\right\rangle$. Our differential operator is simply a generator of $\operatorname{Lie}(G)$.

Back to the flag variety. Since $\mathfrak{n}^{0}$ acts trivially on $\mathcal{O}_{K^{p}}^{\text {la }}$, there is an induced action of $\mathfrak{b}^{0} / \mathfrak{n}^{0} \cong \mathfrak{h} \otimes \mathcal{O}_{\mathscr{F} \ell}$, where $\mathfrak{h}$ is a Cartan subalgebra of $\mathfrak{g}$. We choose it as the Levi quotient of $\mathfrak{b}$; that is, the diagonal matrices. Hence, we have an (horizontal) action $\theta_{\mathfrak{h}}$ of $\mathfrak{h}$ on $\mathcal{O}_{K^{p}}^{\text {la }}$. By Harish-Chandra's theory, this action is closely related to the action of the centre $Z(U(\mathfrak{g}))$ (Corollary 4.2.8). In the study of cohomology of locally symmetric spaces, the infinitesimal group action is related to Hodge theory. Our next result gives a $p$-adic Hodgetheoretic interpretation of $\theta_{\mathfrak{h}}$.

Theorem 1.0.14 (Theorem 5.1.11). $\left.\theta_{\mathfrak{h}}\left(\begin{array}{ll}0 & 0 \\ 0 & 1\end{array}\right)\right)$ is the unique Sen operator on the cohomology $H^{i}\left(\mathscr{F} \ell, \mathcal{O}_{K^{p}}^{\mathrm{la}}\right) \cong \tilde{H}^{i}\left(K^{p}, C\right)^{\mathrm{la}}$ for any $i$.

Remark 1.0.15. Since the usual Sen operator is defined for a finite-dimensional $C$-semilinear representation of $G_{\mathbb{Q}_{p}}$, we will generalise this notion to this situation in Definition 5.1.5. For example, for any finite-dimensional $C$-semilinear subrepresentation $V$ of $G_{\mathbb{Q}_{p}}$ in $\tilde{H}^{i}\left(K^{p}, C\right)^{\text {la }}$, this result implies that $\left.\theta_{\mathfrak{h}}\left(\begin{array}{ll}0 & 0 \\ 0 & 1\end{array}\right)\right)$ agrees with usual Sen operator when restricted to $V$. We also sketch a more direct construction of the Sen operator on $\tilde{H}^{i}\left(K^{p}, C\right)^{\text {la }}$ in Remark 5.1.16. 
Remark 1.0.16. In Beilinson-Bernstein's theory of localisation (see Section C of [Beř84]), $\mathcal{O}_{K^{p}}^{\text {la }}$ is a $\tilde{\mathscr{D}}$-module. Note that everything here is also $\mathrm{GL}_{2}\left(\mathbb{Q}_{p}\right)$-equivariant. Hence, we obtain a $\left(\tilde{\mathscr{D}}, \mathrm{GL}_{2}\left(\mathbb{Q}_{p}\right)\right)$ module on $\mathscr{F} \ell$, which is very similar to the picture in the complex analytic setting (for example, the work of Kashiwara-Schmid [KS94]).

As a corollary, this implies that on the locally analytic vectors in the completed cohomology, the infinitesimal character of $\mathrm{GL}_{2}\left(\mathbb{Q}_{p}\right)$ (i.e., action of $Z(U(\mathfrak{g}))$ ) and the infinitesimal character of $G_{\mathbb{Q}_{p}}$ (Sen operator as an element in the centre of ' $C \otimes_{\mathbb{Q}_{p}} \operatorname{Lie}\left(G_{\mathbb{Q}_{p}}\right)$ ') are closely related. This directly implies the (Hodge-Tate) decomposition in Theorem 1.0.1.

Remark 1.0.17. The same strategy of this article should give Theorem 1.0.11 for higher dimensional rigid analytic varieties, Theorem 1.0.10 for general Hodge-Tate period maps and Theorem 1.0.14 for Shimura varieties of Hodge type. I plan to report about this in a future paper. We also note that the relation between the action of the centre of the universal enveloping algebra and Sen operator on the locally analytic vectors in the completed cohomology (not necessarily of the modular curves) was already vastly studied by Dospinescu-Paškūnas-Schraen in [DPS20].

After writing up this article, I was informed by Sean Howe that very recently he also obtained some part of Theorem 5.4.2 and the easier direction of Theorem 1.0.7 in [How21].

For people who are interested in the relation between our work and the higher Coleman theory recently developed by Boxer-Pilloni in [BP21], it is worth pointing out that one can show that the $N_{k, 1}(k \geq 2)$ in Theorem 1.0.1 and $N_{1}$ in Theorem 1.0.2 are nothing but the direct limit of the first cohomology of $\omega^{k}$ on $\mathcal{X}_{K^{p} \Gamma\left(p^{n}\right)}$ with support in the closure (in the adic topology) of $\mathcal{X}_{K^{p} \Gamma\left(p^{n}\right), c}$. See also [Cam21, Theorem 1.0.3].

This article is organised as follows. We first collect some basic facts about locally analytic vectors in Section 2. One important notion is a derived functor $R^{i} \mathfrak{Q A}$ of taking locally analytic vectors introduced in Definition 2.2.1.

In Section 3, we explain how to derive the differential equation claimed in Theorem 1.0.11 from relative Sen theory. Along the way, we also prove a vanishing result for $R^{i} \mathfrak{Q A}$ which later will be used to prove Theorem 1.0.9. Nothing is new in the sense that all of the techniques were probably well-known in Sen's theory.

In Section 4, we apply our theory developed in the previous section to modular curves at infinite level. The computation of the differential equation in this case is a simple reinterpretation of an old result of Faltings. We also give an explicit description of the sections of $\mathcal{O}_{K^{p}}^{\text {la }}$ and use it to prove Theorem 1.0.9.

In Section 5, we first give a proof of Theorem 1.0.14. Using our explicit description of $\mathcal{O}_{K^{p}}^{\mathrm{la}}$, it is easy to verify that $\theta_{\mathfrak{h}}\left(\begin{array}{ll}0 & 0 \\ 0 & 1\end{array}\right)$ ) behaves as a Sen operator on $\mathcal{O}_{K^{p}}^{\text {la }}$ and its cohomology. Next we compute $\tilde{H}^{1}\left(K^{p}, C\right)_{\mu}^{\text {la }}$. Fix a character $\chi \in \mathfrak{h}^{*}$ and denote by $\mathcal{O}_{K^{p}}^{\text {la, } \chi}$ the $\theta_{\mathfrak{h}}=\chi$-isotypic part. Let $\mathfrak{n}$ be the nilpotent subalgebra of $\mathfrak{b}$. Basically we are reduced to computing the $\mathfrak{n}$-cohomology of the sheaf $\mathcal{O}_{K^{p}}^{\mathrm{la}, \chi}$ and the cohomology of the $\mathfrak{n}$-invariants and $\mathfrak{n}$-coinvariants of $\mathcal{O}_{K^{p}}^{\text {la, } \chi}$ on $\mathscr{F} \ell$ (at least for nonsingular weight).

In Section 6, we give the global applications mentioned earlier. To do this, we need Emerton's work on local-global compatibility and Colmez's work on Kirillov models. It should be mentioned that we slightly generalise Emerton's local-global compatibility result so that it is now valid when localised at a Eisenstein maximal ideal.

\section{Notation}

Fix an algebraic closure $\overline{\mathbb{Q}}$ of $\mathbb{Q}$. Denote by $G_{\mathbb{Q}}$ the absolute Galois group $\operatorname{Gal}(\overline{\mathbb{Q}} / \mathbb{Q})$. For each rational prime $l$, fix an algebraic closure $\overline{\mathbb{Q}}_{l}$ of $\mathbb{Q}_{l}$ with ring of integers $\overline{\mathbb{Z}}_{l}$, an embedding $\overline{\mathbb{Q}} \rightarrow \overline{\mathbb{Q}}_{l}$ which determines a decomposition group $G_{\mathbb{Q}_{l}} \subset G_{\mathbb{Q}}$ at $l$ and a lift of geometric Frobenius Frob $\in_{l} \in G_{\mathbb{Q}_{l}}$. Our convention for local class field theory sends a uniformiser to a lift of geometric Frobenius. We denote by 
$\varepsilon$ the $p$-adic cyclotomic character $G_{\mathbb{Q}} \rightarrow \mathbb{Z}_{p}^{\times}$and, by abuse of notation, also the corresponding character $\mathbb{A}_{f}^{\times} / \mathbb{Q}_{>0}^{\times} \rightarrow \mathbb{Z}_{p}^{\times}$via global class field theory. Similarly, we denote by $\varepsilon_{p}$ the $p$-adic cyclotomic character $G_{\mathbb{Q}_{p}} \rightarrow \mathbb{Z}_{p}^{\times}$and also the character $\mathbb{Q}_{p}^{\times} \rightarrow \mathbb{Z}_{p}^{\times}$sending $x$ to $x|x|$. Suppose $S$ is a finite set of rational primes. We denote by $G_{\mathbb{Q}, S}$ the the Galois group of the maximal extension of $\mathbb{Q}$ unramified outside $S$ and $\infty$.

All completed tensor products mean ' $p$-adically completed tensor product'. For example, if $V$ and $W$ are two $\mathbb{Q}_{p}$-Banach spaces, then $V \widehat{\otimes}_{\mathbb{Q}_{p}} W=\left(\lim _{n}\left(V^{o} \otimes_{\mathbb{Z}_{p}} W^{o} / p^{n}\right)\right) \otimes_{\mathbb{Z}_{p}} \mathbb{Q}_{p}$, where $V^{o}$ and $W^{o}$ denote the open unit balls of $V$ and $W$.

If $\rho: \Gamma \rightarrow \mathrm{GL}_{n}\left(\overline{\mathbb{Q}}_{p}\right)$ denotes a continuous representation of a profinite group $\Gamma$, we denote by $\bar{\rho}^{s s}: \Gamma \rightarrow \mathrm{GL}_{2}\left(\overline{\mathbb{F}}_{p}\right)$ the semi-simplification of its residual representation, which is well-defined up to conjugation. If $\bar{\rho}^{s s}$ is irreducible, we simply write $\bar{\rho}$. Here $\overline{\mathbb{F}}_{p}$ denotes the residue field of $\overline{\mathbb{Z}}_{p}$.

For an adic space $X$, an open set $V \subset X$ and a sheaf $\mathcal{F}$ on $X$, we sometimes write $\mathcal{F}(V)$ instead of $H^{0}(\mathcal{V}, \mathcal{F})$. For example, $\mathcal{O}_{X}(V)$ denotes analytic functions on $V$.

For a tuple $\mathbf{k}=\left(k_{1}, \cdots, k_{d}\right) \in \mathbb{N}^{d}$, we denote the sum $\sum_{i=1}^{d} k_{i}$ by $|\mathbf{k}|$.

Suppose $G$ is a group and $f$ is a function on $G$ (valued in some group). Let $g \in G$. The left translation action of $g$ on $f$ is denoted by ${ }^{l} g \cdot f$, which sends $x \in G$ to $f\left(g^{-1} x\right)$. Similarly, the right translation action of $g$ on $f$ is denoted by ${ }^{r} g \cdot f$ and sends $x \in G$ to $f(x g)$. Note that both actions are left actions.

\section{Locally analytic vectors}

In this section, we collect some basic results in the theory of locally analytic representations studied by Schneider and Teitelbaum [ST02, ST03]. We will first recall the (naive) definition of locally analytic functions on a compact $p$-adic Lie group, following Subsection 2.1 of [BC16]. Then we will introduce a derived functor of taking locally analytic vectors.

\subsection{Definition and basic properties}

2.1.1. Let $G$ be a $p$-adic Lie group of dimension $d$. By Theorem 27.1 of [Sch11], there exists a compact open subgroup $G_{0}$ of $G$ equipped with an integer-valued, saturated $p$-valuation. As a consequence, there exist $g_{1}, \cdots, g_{d} \in G_{0}$ (an ordered basis) such that the map $c: \mathbb{Z}_{p}^{d} \rightarrow G_{0}$ defined by $\left(x_{1}, \cdots, x_{d}\right) \mapsto$ $g_{1}^{x_{1}} \cdots g_{d}^{x_{d}}$ is a homeomorphism. Let $G_{n}=G_{0}^{p^{n}}=\left\{g^{p^{n}}, g \in G_{0}\right\}$ for $n \geq 1$. This is in fact a subgroup, and $c^{-1}\left(G_{n}\right)=\left(p^{n} \mathbb{Z}_{p}\right)^{d}$. So $\left\{G_{n}\right\}_{n}$ forms a fundamental system of open neighbourhoods of the identity element 1 in $G$. We will fix such a $c$ in the following discussion, but all of the definitions we make below will not depend on the choice of $c$. See Section 23 and Section 26 of [Sch11] for more details.

If $B$ is a $\mathbb{Q}_{p}$-Banach space with norm $\|\cdot\|$, we denote by $\mathscr{C}\left(G_{n}, B\right)$ the space of $B$-valued continuous functions on $G_{n}$ and by $\mathscr{C}^{\text {an }}\left(G_{n}, B\right)$ the subspace of $B$-valued analytic functions. More precisely, $f: G_{n} \rightarrow B$ is analytic if its pullback under $c$ can be written as

$$
\mathbf{x}=\left(x_{1}, \cdots, x_{d}\right) \in\left(p^{n} \mathbb{Z}_{p}\right)^{d} \mapsto \sum_{\mathbf{k}=\left(k_{1}, \cdots, k_{d}\right) \in \mathbb{N}^{d}} b_{\mathbf{k}} x_{1}^{k_{1}} \cdots x_{d}^{k_{d}}
$$

for some $b_{\mathbf{k}} \in B$ such that $p^{n|\mathbf{k}|} b_{\mathbf{k}} \rightarrow 0$ as $|\mathbf{k}| \rightarrow \infty$. We define $\|f\|_{G_{n}}=\sup _{\mathbf{k} \in \mathbb{N} d}\left\|p^{n|\mathbf{k}|} b_{\mathbf{k}}\right\|$ and $\mathscr{C}^{\text {an }}\left(G_{n}, B\right)$ is a $\mathbb{Q}_{p}$-Banach space under this norm. There is a natural norm-preserving isomorphism $\mathscr{C}^{\text {an }}\left(G_{n}, \mathbb{Q}_{p}\right) \widehat{\otimes}_{\mathbb{Q}_{p}} B \cong \mathscr{C}^{\text {an }}\left(G_{n}, B\right)$ induced by the $\mathbb{Q}_{p}$-linear structure of $B$. When $B=\mathbb{Q}_{p}$, the usual point-wise multiplication of two functions defines a natural $\mathbb{Q}_{p}$-algebra structure on $\mathscr{C}^{\text {an }}\left(G_{n}, \mathbb{Q}_{p}\right)$.

To see this definition is independent of the choice of $g_{1}, \cdots, g_{d}$, we introduce another set of coordinates of $G_{n}$ given by the the exponential map. These coordinates are called coordinates of the first kind in Section 34 of [Sch11]. More precisely, let $\operatorname{Lie}(G)$ be the Lie algebra of $G$ and consider the logarithm map

$$
\log : G_{0} \rightarrow \operatorname{Lie}(G)
$$


which is a homeomorphism onto its image. In fact, its image $\log \left(G_{0}\right)$ is a free $\mathbb{Z}_{p}$-module with a basis $\log \left(g_{1}\right), \cdots, \log \left(g_{d}\right)$. Hence,

$$
e: \mathbb{Z}_{p}^{d} \rightarrow G_{0},\left(y_{1}, \cdots, y_{d}\right) \mapsto \exp \left(y_{1} \log \left(g_{1}\right)+\cdots+y_{d} \log \left(g_{d}\right)\right)
$$

defines a homeomorphism. Here exp is the inverse of log (exponential map). Under this parametrisation, $G_{n}$ is identified with the image of $\left(p^{n} \mathbb{Z}_{p}\right)^{d}$. As before, a function on $G_{n}$ is called analytic if its pullback under $e$ can be written as a power series in $y_{i}$ with certain growth conditions on its coefficients. This definition of analyticity agrees with the previous definition as the transition functions between the coordinates $\left(x_{1}, \cdots, x_{d}\right)$ and $\left(y_{1}, \cdots, y_{d}\right)$ are given by convergent power series with coefficients in $\mathbb{Z}_{p}$; cf. Proposition 34.1 of [Sch11].

Different choices of ordered bases $g_{1}, \cdots, g_{d}$ give rise to different bases of $\log \left(G_{0}\right)$ over $\mathbb{Z}_{p}$ (as a $\mathbb{Z}_{p}$-module). It is easy to see now that $\mathscr{C}^{\text {an }}\left(G_{n}, B\right)$ and $\|\cdot\|_{G_{n}}$ are independent of choice of an ordered basis. As a corollary, $\mathscr{C}^{\text {an }}\left(G_{n}, B\right)$ and $\|\cdot\|_{G_{n}}$ are invariant under both the left and right translation actions of $G_{0}$. We sketch a proof for the left translation by $G_{0}$ here. The case for right translation and general $n$ will be exactly the same. Let $x$ be a nontrivial element of $G_{0}$. It suffices to prove the claim under the assumption that $x$ is not a $p$ th power. Choose an ordered basis $g_{1}, \cdots, g_{d}$ with $g_{1}=x$. This is possible by our assumption on $G_{0}$; see Section 26 of [Sch11]. To compute the left translation action of $x$, we are essentially reduced to the case $G_{0}=\mathbb{Z}_{p}$, which can be verified directly. In fact, this argument shows a bit more.

Lemma 2.1.2. For any integer $n \geq 0$, let $\mathscr{C}^{\mathrm{an}}\left(G_{n}, \mathbb{Q}_{p}\right)^{o} \subset \mathscr{C}^{\mathrm{an}}\left(G_{n}, \mathbb{Q}_{p}\right)$ be the unit ball with respect to the norm $\|\cdot\|_{G_{n}}$. Then both left and right translation actions of $G_{n+1}$ are trivial on $\mathscr{C}^{\mathrm{an}}\left(G_{n}, \mathbb{Q}_{p}\right)^{o} / p$.

Proof. One can reduce to the trivial case $G_{n}=\mathbb{Z}_{p}$ by the same argument.

The following density result will be used later.

Proposition 2.1.3. For $n$ large enough, there is a dense subspace $\lim _{k \in \mathbb{N}} V_{k} \subset \mathscr{C}^{\mathrm{an}}\left(G_{n}, \mathbb{Q}_{p}\right)$, where each $V_{k}$ is a finite-dimensional subspace of $\mathscr{C}^{\mathrm{an}}\left(G_{n}, \mathbb{Q}_{p}\right)$ stable under both the left and right translation actions of $G_{n}$ and such that for any $k, l \in \mathbb{N}, f_{k} \in V_{k}, f_{l} \in V_{l}$, we have $f_{k} f_{l} \in V_{k+l}$.

Proof. This is essentially proved in the proof of Theorem 6.1 of [BC16]. We recall their argument here. The rough idea is to reduce to the case $G=\operatorname{GL}_{N}\left(\mathbb{Z}_{p}\right)$, in which case the algebraic functions on $\mathrm{GL}_{N}$ are dense in the space of analytic functions.

For $n$ large enough, we may find an embedding $G_{n}$ into $1+p^{2} M_{N}\left(\mathbb{Z}_{p}\right) \subset \mathrm{GL}_{N}\left(\mathbb{Z}_{p}\right)$ for some $N$, where $M_{N}\left(\mathbb{Z}_{p}\right)$ denotes the space of $N \times N$ matrices over $\mathbb{Z}_{p}$. We sketch a proof of this well-known result here because it seems to lack a suitable reference. By Ado's theorem (Section 7, 3, Theorem 3 of [Bou60]), there is a faithful representation of $\operatorname{Lie}(G)$ over $\mathbb{Q}_{p}^{\oplus N}$ for some $N$. Choose $n$ large enough so that $\log \left(G_{n}\right)$ maps to $p^{2} M_{N}\left(\mathbb{Z}_{p}\right)$. Then this Lie algebra morphism can be integrated to a group homomorphism $G_{n} \rightarrow 1+p^{2} M_{N}\left(\mathbb{Z}_{p}\right)$, which is necessarily a closed embedding. This can be deduced from results in Section 31, notably Theorem 31.5 of [Sch11].

Fix such an embedding. $G_{n}$ is now viewed as a subset of $M_{N}\left(\mathbb{Q}_{p}\right)$. For $k \in N$, let $V_{k}$ be the space of $\mathbb{Q}_{p}$-valued functions on $G_{n}$ that are restrictions of polynomials of degree $\leq k$ on $M_{N}\left(\mathbb{Q}_{p}\right)$. Here we identify $M_{N}\left(\mathbb{Q}_{p}\right)$ with an affine space of dimension $N^{2}$ (over $\mathbb{Q}_{p}$ ). We claim that the union of all $V_{k}$ is dense in $\mathscr{C}^{\text {an }}\left(G_{n}, \mathbb{Q}_{p}\right)$. To see this, note that the space of polynomial functions on $M_{N}\left(\mathbb{Q}_{p}\right)$ is dense in $\mathscr{C}^{\text {an }}\left(1+p^{2} M_{N}\left(\mathbb{Z}_{p}\right), \mathbb{Q}_{p}\right)$ when restricted to $1+p^{2} M_{N}\left(\mathbb{Z}_{p}\right)$. Hence, its pullback under the exponential map is dense in $\mathscr{C}^{\text {an }}\left(p^{2} M_{N}\left(\mathbb{Z}_{p}\right), \mathbb{Q}_{p}\right)$. As the image of $\mathscr{C}^{\text {an }}\left(p^{2} M_{N}\left(\mathbb{Z}_{p}\right), \mathbb{Q}_{p}\right) \rightarrow \mathscr{C}^{\text {an }}\left(\log \left(G_{n}\right), \mathbb{Q}_{p}\right)$ is dense, this implies our claim.

It is clear from the construction that all $V_{k}$ are stable under left and right translation actions and closed under multiplications. 
2.1.4. Now if $B$ is a $\mathbb{Q}_{p}$-Banach space equipped with a continuous action of $G_{n}$, then we denote by $B^{G_{n} \text {-an }} \subset B$ the subset of $G_{n}$-analytic vectors. One can define this as follows: there is a left action on $\mathscr{C}^{\text {an }}\left(G_{n}, B\right)$ by $(g \cdot f)(h)=g\left(f\left(g^{-1} h\right)\right), g, h \in G_{n}, f \in \mathscr{C}^{\text {an }}\left(G_{n}, B\right)$. We define $B^{G_{n} \text {-an }}$ as the image of $\mathscr{C}^{\text {an }}\left(G_{n}, B\right)^{G_{n}} \rightarrow B$ under the evaluation map at the identity element in $G_{n}$. Equivalently, $v \in B^{G_{n}-\text { an }}$ if $f_{v}: G_{n} \rightarrow B, f_{v}(g)=g \cdot v$ lies in $\mathscr{C}^{\text {an }}\left(G_{n}, B\right)$. It is clear that $\|\cdot\|_{G_{n}}$ induces a norm on $B^{G_{n}-\mathrm{an}}$, which we denote by the same notation. Now $G_{n}$ acts on $B^{G_{n}-\text { an }}=\mathscr{C}^{\text {an }}\left(G_{n}, B\right)^{G_{n}}$ by right translation. One checks easily that this action is unitary and equivariant with respect to the inclusion $B^{G_{n}-\text { an }} \subset B$.

We say $B$ is an analytic representation of $G_{n}$ if $B=B^{G_{n}-\text { an }}$.

One has the following lemma (cf. Lemme 2.4. of [BC16]).

Lemma 2.1.5. Let $B$ be a Banach representation of $G_{n}$. If $b \in B^{G_{n}-\mathrm{an}}$, then

(1) $b \in B^{G_{n+1}-\text { an }}$,

(2) $\|b\|_{G_{n+1}} \leq\|b\|_{G_{n}}$.

(3) $\|b\|_{G_{m}}$ agrees with the norm of $b$ in $B$ for $m$ sufficiently large.

Definition 2.1.6. Suppose $B$ is a Banach representation of $G_{0}$. The space of locally analytic vectors in $B$ is $B^{\text {la }}=\bigcup_{n \geq 0} B^{G_{n}-\text { an }}$ with the direct limit topology.

Remark 2.1.7. If $B$ is a representation of $G$, then $B^{\text {la }}$ can be defined by restricting to the action of $G_{0}$. It is easy to see that $B^{\text {la }}$ is independent of the choice of $G_{0}$ and $B^{\text {la }}$ is $G$-stable.

The Lie algebra $\operatorname{Lie}(G)$ acts on the locally analytic vectors. One can check this in the universal case $\mathscr{C}^{\text {an }}\left(G_{n}, \mathbb{Q}_{p}\right)$. Indeed, one writes $B^{G_{n} \text { an }}=\left(\mathscr{C}^{\text {an }}\left(G_{n}, \mathbb{Q}_{p}\right) \widehat{\otimes}_{\mathbb{Q}_{p}} B\right)^{G_{n}}$. The Lie algebra action of $\operatorname{Lie}(G)$ on $B^{G_{n}-\text { an }}$ comes from the action on $\mathscr{C}^{\text {an }}\left(G_{n}, \mathbb{Q}_{p}\right)$ induced from the right translation action. The following lemma is Lemme 2.6 of [BC16].

Lemma 2.1.8. Suppose $B$ is a Banach representation of $G_{0}$. If $D \in \operatorname{Lie}(G)$ and $n \geq 1$, then there exists a constant $C_{D, n}$ such that $\|D(x)\|_{G_{n}} \leq C_{D, n}\|x\|_{G_{n}}$ for any $x \in B^{G_{n}-\mathrm{an}}$.

The following example will appear naturally later.

Example 2.1.9. Suppose $G=G_{0}=\mathbb{Z}_{p}$ and $B$ is a $\mathbb{Q}_{p}$-Banach space representation of $\mathbb{Z}_{p}$. Let $B^{o}$ be the open unit ball of $B$. Suppose there exists an integer $m>0$ such that

$$
(\gamma-1)^{m} \cdot\left(B^{o}\right) \subset p B^{o}
$$

for any $\gamma \in \mathbb{Z}_{p}$. Then $B$ is an analytic representation of $p^{h} \mathbb{Z}_{p}$ for $h$ sufficiently large. To see this, it suffices to prove that $B$ is an analytic representation of $\mathbb{Z}_{p}$ if $(\gamma-1) \cdot\left(B^{o}\right) \subset p^{2} B^{o}$ for any $\gamma \in \mathbb{Z}_{p}$. Let $\gamma=1 \in \mathbb{Z}_{p}$. For any $f \in B$, consider $g: \mathbb{Z}_{p} \rightarrow B$ defined by $g(x)=\sum_{n=0}^{+\infty}\left(\begin{array}{l}x \\ n\end{array}\right)(\gamma-1)^{n} \cdot f$. One checks easily that $g$ is analytic and $g(x)=x \cdot f$. Hence, $f \in B^{\mathbb{Z}_{p}-\text { an }}$ and $B=B^{\mathbb{Z}_{p}-\text { an }}$.

\subsection{Derived functor}

Let $G, G_{0}, G_{n}$ be as in the first paragraph of Section 2.1.1 and let $B$ be a $\mathbb{Q}_{p}$-Banach representation of $G$. Recall

$$
B^{\mathrm{la}}=\underset{n}{\lim } B^{G_{n}-\mathrm{an}}=\underset{n}{\lim }\left(B \widehat{\otimes}_{\mathbb{Q}_{p}} \mathscr{C}^{\mathrm{an}}\left(G_{n}, \mathbb{Q}_{p}\right)\right)^{G_{n}}=\underset{n}{\lim } H_{\mathrm{cont}}^{0}\left(G_{n}, B \widehat{\otimes}_{\mathbb{Q}_{p}} \mathscr{C}^{\mathrm{an}}\left(G_{n}, \mathbb{Q}_{p}\right)\right)
$$

Write $\mathfrak{Q A}(B)=B^{\text {la }}$. We can also consider the following 'right derived functor' of $\mathfrak{R} \mathfrak{A}$.

Definition 2.2.1. For a $\mathbb{Q}_{p}$-Banach representation $B$ of $G$, let

$$
R^{i} \mathfrak{Q} \mathfrak{A}(B):=\underset{n}{\lim } H_{\text {cont }}^{i}\left(G_{n}, B \widehat{\otimes}_{\mathbb{Q}_{p}} \mathscr{C}^{\text {an }}\left(G_{n}, \mathbb{Q}_{p}\right)\right) .
$$


We say $B$ is

○ $\mathfrak{Q P}$-acyclic if $R^{i} \mathfrak{Q A}(B)=0, i \geq 1$;

○ strongly $\mathfrak{R} \mathfrak{A}$-acyclic if the direct systems $\left\{H_{\text {cont }}^{i}\left(G_{n}, B \widehat{\otimes}_{\mathbb{Q}_{p}} \mathscr{C}^{\text {an }}\left(G_{n}, \mathbb{Q}_{p}\right)\right)\right\}_{n}, i \geq 1$ are essentially zero; that is, for any $n$, the image of $H_{\text {cont }}^{i}\left(G_{n}, B \widehat{\otimes}_{\mathbb{Q}_{p}} \mathscr{C}^{\text {an }}\left(G_{n}, \mathbb{Q}_{p}\right)\right) \rightarrow H_{\text {cont }}^{i}\left(G_{m}, B \widehat{\otimes}_{\mathbb{Q}_{p}} \mathscr{C}^{\text {an }}\right.$ $\left.\left(G_{m}, \mathbb{Q}_{p}\right)\right)$ is zero for sufficiently large $m \geq n$.

We put a quotation mark on right derived functor as we do not plan to discuss the abelian category we are working with. Clearly, $R^{i} \mathfrak{Q A}$ measures the failure of exactness when taking the locally analytic vectors and does not depend on choices of $G_{0}$. This $a d$ hoc definition will be enough for our purpose in view of the following simple lemma.

\section{Lemma 2.2.2.}

(1) Suppose $0 \rightarrow M^{0} \rightarrow M^{1} \rightarrow M^{2} \rightarrow 0$ is a short exact sequence of $\mathbb{Q}_{p}$-Banach representations of $G$ with G-equivariant continuous homomorphisms (which are necessarily strict by the open mapping theorem). Then there is a long exact sequence:

$$
0 \rightarrow\left(M^{0}\right)^{\mathrm{la}} \rightarrow\left(M^{1}\right)^{\mathrm{la}} \rightarrow\left(M^{2}\right)^{\mathrm{la}} \rightarrow R^{1} \mathfrak{L} \mathfrak{A}\left(M^{0}\right) \rightarrow R^{1} \mathfrak{L A}\left(M^{1}\right) \rightarrow R^{1} \mathfrak{L} \mathfrak{A}\left(M^{2}\right) \rightarrow \ldots
$$

(2) Let $M^{\bullet}$ be a bounded chain complex of $\mathbb{Q}_{p}$-Banach representations of $G$ with $G$-equivariant strict homomorphisms. If $M^{q}$ and $H^{q}\left(M^{\bullet}\right)$ are $\mathfrak{Q} \mathfrak{A}$-acyclic for any $q$, then $\left(H^{q}\left(M^{\bullet}\right)\right)^{\mathrm{la}}=H^{q}\left(\left(M^{\bullet}\right)^{\mathrm{la}}\right)$.

(3) Let $0 \rightarrow B \rightarrow M^{0} \rightarrow M^{1} \rightarrow \cdots$ be an exact chain complex of $\mathbb{Q}_{p}$-Banach representations of $G$ with $G$-equivariant strict homomorphisms. Assume $M^{q}$ is $\mathfrak{Q A}$-acyclic for any $q$. Then $R^{i} \mathfrak{Q A}(B)=H^{i}\left(\left(M^{\bullet}\right)^{\mathrm{la}}\right)$. Moreover, if all $M^{q}$ are strongly $\mathfrak{Q A}$-acyclic and the direct systems $\left\{H^{i}\left(\left(M^{\bullet}\right)^{G_{n}-\text { an }}\right)\right\}_{n}, i \geq 1$ are essentially zero, then $B$ is strongly $\mathfrak{Q} \mathfrak{A}$-acyclic.

Proof. For the first part, since all of the homomorphisms are strict, $H^{i}\left(M^{\bullet}, o\right)$ can be killed by a bounded power of $p$, where $M^{\bullet}, o$ denotes the open unit ball of $M^{\bullet}$. Therefore, $M^{\bullet} \widehat{\otimes}_{\mathbb{Q}_{p}} \mathscr{C}^{\text {an }}\left(G_{n}, \mathbb{Q}_{p}\right)$ remains exact for any $n$ and we obtain the desired long exact sequence by taking $G_{n}$-cohomology and passing to the direct limit over $n$.

The second and third parts follow from the first part by a simple induction argument. For example, one can prove that the kernel and image of $M^{q} \rightarrow M^{q+1}$ are $\mathfrak{Q} \mathfrak{A}$-acyclic by induction on $q$. This certainly implies that $\left(H^{q}\left(M^{\bullet}\right)\right)^{\mathrm{la}}=H^{q}\left(\left(M^{\bullet}\right)^{\mathrm{la}}\right)$. We leave the third part as an exercise.

A result of Schneider-Teitelbaum says that admissible representations are $\mathfrak{Q A}$-acyclic (Theorem 7.1 of [ST03]). In fact, their argument also essentially proves strong $\mathfrak{Q} \mathfrak{A}$-acyclicity.

Theorem 2.2.3. Any admissible $\mathbb{Q}_{p}$-Banach representation $B$ of $G$ is strongly $\mathfrak{Q} \mathfrak{A}$-acyclic.

Proof. Since $B$ is admissible, there is a $G_{0}$-equivariant embedding $B \hookrightarrow \mathscr{C}\left(G_{0}, \mathbb{Q}_{p}\right)^{\oplus d}$ for some $d$. Then the quotient $C$ is an admissible $\mathbb{Q}_{p}$-Banach representation of $G_{0}$. Note that $\mathscr{C}\left(G_{0}, \mathbb{Q}_{p}\right) \cong \mathscr{C}\left(G_{n}, \mathbb{Q}_{p}\right)^{\oplus d_{n}}$ as a representation of $G_{n}$ for some $d_{n}$. It follows from Shapiro's lemma for continuous cohomology that $H_{\text {cont }}^{1}\left(G_{n}, \mathscr{C}\left(G_{0}, \mathbb{Q}_{p}\right) \widehat{\otimes}_{\mathbb{Q}_{p}} \mathscr{C}^{\text {an }}\left(G_{n}, \mathbb{Q}_{p}\right)\right)=0$. Hence,

$$
\left(\mathscr{C}\left(G_{0}, \mathbb{Q}_{p}\right)^{\oplus d}\right)^{G_{n} \text { an }} \rightarrow C^{G_{n}-\mathrm{an}} \rightarrow H_{\text {cont }}^{1}\left(G_{n}, B \widehat{\otimes}_{\mathbb{Q}_{p}} \mathscr{C}^{\text {an }}\left(G_{n}, \mathbb{Q}_{p}\right)\right) \rightarrow 0 .
$$

By Corollaire IV.14. of [CD14], we have an exact sequence

$$
0 \rightarrow B^{(m)} \rightarrow\left(\mathscr{C}\left(G_{0}, \mathbb{Q}_{p}\right)^{\oplus d}\right)^{(m)} \rightarrow C^{(m)} \rightarrow 0
$$

See [CD14, §IV] for the notation here. One can check $C^{G_{n}-\mathrm{an}} \subset C^{(n+2)} \subset C^{G_{n+1}-\mathrm{an}}$. This means that the image of $\left(\mathscr{C}\left(G_{0}, \mathbb{Q}_{p}\right)^{\oplus d}\right)^{G_{n+1} \text { an }}$ in $C^{G_{n+1}-\text { an }}$ contains $C^{G_{n}-\text { an }}$. Hence, $H_{\text {cont }}^{1}\left(G_{n}, B \widehat{\otimes}_{\mathbb{Q}_{p}} \mathscr{C}^{\text {an }}\left(G_{n}, \mathbb{Q}_{p}\right)\right) \rightarrow H_{\text {cont }}^{1}\left(G_{n+1}, B \widehat{\otimes}_{\mathbb{Q}_{p}} \mathscr{C}^{\text {an }}\left(G_{n+1}, \mathbb{Q}_{p}\right)\right)$ has zero image. A simple induction on cohomology degree $i \geq 1$ proves the theorem.

We need the following variant for later applications. 
Corollary 2.2.4. Let $B$ be an admissible $\mathbb{Q}_{p}$-Banach representation of $G$ and $M$ be a $\mathbb{Q}_{p}$-Banach space with trivial G-action. Then $B \widehat{\otimes}_{\mathbb{Q}_{p}} M$ is strongly $\mathfrak{Q} \mathfrak{A}$-acyclic.

Proof. Let $B^{o}$ be the unit open ball of $B$. Take $n$ large enough so that $B^{o}$ is $G_{n}$-stable. We claim that $H_{\text {cont }}^{\bullet}\left(G_{n}, B^{o}\right)$ is a finitely generated $\mathbb{Z}_{p}$-module and hence has bounded $p$-torsion. Indeed, since $B$ is admissible, there exists a $\mathbb{Z}_{p}\left[G_{n}\right]$-equivariant injection $B^{o} \hookrightarrow \mathscr{C}\left(G_{n}, \mathbb{Z}_{p}\right)^{\oplus d}$ for some $d$, whose quotient is $p$-torsion free. An induction argument on $i$ implies the finiteness here. It is well-known that $H_{\text {cont }}^{\bullet}\left(G_{n}, B\right)$ can be computed by a cochain complex $\left(\mathscr{C}\left(G_{n}, B\right)^{\oplus i}, d^{i}\right)$. This is a strict complex because $\left(\mathscr{C}\left(G_{n}, B^{o}\right)^{\oplus i}, d^{i}\right)$ computes $H_{\text {cont }}^{\bullet}\left(G_{n}, B^{o}\right)$. Since $\mathscr{C}\left(G_{n}, B \widehat{\otimes}_{\mathbb{Q}_{p}} M\right) \cong \mathscr{C}\left(G_{n}, B\right) \widehat{\otimes}_{\mathbb{Q}_{p}} M$, we know that $\left(\mathscr{C}\left(G_{n}, B\right)^{\oplus i} \widehat{\otimes}_{\mathbb{Q}_{p}} M, d^{i} \otimes \mathbf{1}\right)$ computes $H_{\text {cont }}^{\bullet}\left(G_{n}, B \widehat{\otimes}_{\mathbb{Q}_{p}} M\right)$. Therefore,

$$
H_{\text {cont }}^{\bullet}\left(G_{n}, B \widehat{\otimes}_{\mathbb{Q}_{p}} M\right) \cong H_{\text {cont }}^{\bullet}\left(G_{n}, B\right) \widehat{\otimes}_{\mathbb{Q}_{p}} M .
$$

Our claim now follows from the previous theorem.

\section{Locally analytic vectors and relative Sen theory}

The main goal of this section is to generalise results of Berger and Colmez [BC16] and Sen [Sen81] in the 1-dimensional geometric setting. The main result roughly says that the locally analytic vectors satisfy a differential equation given by a (relative) Sen operator. We claim no originality for most results here. One strong tool in these works is the theory of decompletions. We will review the Tate-Sen formalism of Berger-Colmez [BC08], which is flexible enough to handle our situation. As an application, we will show that taking locally analytic vectors is exact under certain conditions.

The results of this section are closely related to the work of Faltings [Fal05], Abbes-Gros-Tsuji [AGT16] and Liu-Zhu [LZ17] on p-adic Simpson correspondences. See Remark 3.1.7.

\subsection{Statement of the main result}

3.1.1. Fix a complete algebraically closed non-Archimedean field $C$ of characteristic zero. Recall that a non-Archimedean field is a topological field whose topology is induced by a non-Archimedean norm $|\cdot|: C \rightarrow \mathbb{R}_{\geq 0}$. It is naturally an extension of $\mathbb{Q}_{p}$ for some $p$ and we assume its norm agrees with the usual $p$-adic norm $|\cdot|_{p}=p^{-\operatorname{val}_{p}(\cdot)}$ on $\mathbb{Q}_{p}$. Let $\mathcal{O}_{C}$ be the ring of integers of $C$. Our setup is as follows:

○ $X=\operatorname{Spa}\left(A, A^{+}\right)$: a 1-dimensional smooth affinoid adic space over $\operatorname{Spa}\left(C, \mathcal{O}_{C}\right)$;

○ $G$ : a finite-dimensional compact $p$-adic Lie group;

- $\widetilde{X}=\operatorname{Spa}\left(B, B^{+}\right)$: an affinoid perfectoid algebra over $\operatorname{Spa}\left(C, \mathcal{O}_{C}\right)$, which is a 'log $G$-Galois pro-étale perfectoid covering' of $X$. More precisely, this means that there is a finite set $S$ of classical points in $X$ and $\widetilde{X} \sim \lim _{i \in I} X_{i}$ in the sense of Definition 7.14 of [Sch12] for some index set $I$, where each $X_{i}=\operatorname{Spa}\left(B_{i}, B_{i}^{+}\right)$is a finite Galois covering of $X$ unramified outside of $S$, and $B^{+}$is the $p$-adic completion of $\lim _{i} B_{i}^{+}$. Moreover, the inverse limit of the Galois group of $X_{i}$ over $X$ is identified with $G$. When $S$ is nonempty, we further assume for each point $s$ in $S$, the ramification-index $e_{i}$ of $X_{i} \rightarrow X$ at $s$ is a $p$-power for any $i$ and $\left\{e_{i}\right\}_{i \in I}$ is unbounded;

$\circ$ assume $X$ is small in the sense that $S$ contains at most one element and there is an étale map $X \rightarrow \mathbb{T}^{1}=\operatorname{Spa}\left(C\left\langle T^{ \pm 1}\right\rangle, \mathcal{O}_{C}\left\langle T^{ \pm 1}\right\rangle\right)$ (respectively $X \rightarrow \mathbb{B}^{1}=\operatorname{Spa}\left(C\langle T\rangle, \mathcal{O}_{C}\langle T\rangle\right)$ mapping $S$ to 0 ) when $S$ is empty (respectively otherwise) which factors as a composite of rational embeddings and finite étale maps.

Note that $G$ acts continuously on the Banach algebra $B$ and we can consider the locally analytic vectors $B^{\text {la }} \subset B$. Let $\operatorname{Lie}(G)$ be the Lie algebra of $G$. It acts on $B^{\text {la }}$, and this action can be extended $B$-linearly to a map $B \otimes_{\mathbb{Q}_{p}} \operatorname{Lie}(G) \times B^{\text {la }} \rightarrow B$. 
Theorem 3.1.2. Fix an étale map $X \rightarrow \mathbb{T}^{1}$ (respectively $X \rightarrow \mathbb{B}^{1}$ ) if $S$ is empty (respectively nonempty) as above. For each 'log G-Galois pro-étale perfectoid covering' $\widetilde{X}$ of $X$, we can assign an element $\theta=\theta_{\widetilde{X}} \in B \otimes_{\mathbb{Q}_{p}} \operatorname{Lie}(G)$, satisfying

(1) $\theta$ annihilates $B^{\text {la }}$. In other words, locally analytic vectors satisfy certain (first-order) differential equation.

(2) $\theta$ is functorial in $(G, \tilde{X})$ : if $H$ is a closed normal subgroup of $G$ so that $\widetilde{X}^{\prime}=\operatorname{Spa}\left(B^{H},\left(B^{+}\right)^{H}\right)$ is a 'log $G / H$-Galois pro-étale perfectoid covering' of $X$, then

$$
\theta_{\widetilde{X}} \equiv \theta_{\widetilde{X}^{\prime}} \bmod B \otimes_{\mathbb{Q}_{p}} \operatorname{Lie}(H),
$$

where $\theta_{\widetilde{X}^{\prime}} \in B^{H} \otimes_{\mathbb{Q}_{p}} \operatorname{Lie}(G / H)$ is viewed as an element in $B \otimes_{\mathbb{Q}_{p}} \operatorname{Lie}(G / H)$.

(3) $\theta \neq 0$ if $\widetilde{X}$ is a locally analytic covering in the sense of 3.5.4.

Moreover, if we start with another étale map $X \rightarrow \mathbb{T}^{1}$ (or étale map $X \rightarrow \mathbb{B}^{1}$ ), then the element $\theta^{\prime} \in B \otimes_{\mathbb{Q}_{p}} \operatorname{Lie}(G)$ obtained using this étale map will differ $\theta$ by a unit of $A$. In other words, $A^{\times} \theta \subset$ $B \otimes \mathbb{Q}_{p} \operatorname{Lie}(G)$ does not depend on the choice of the étale map.

Remark 3.1.3. From the point of view of differential operators, one may regard $\theta$ as some $p$-adic analogue of Cauchy-Riemann operator in the classical complex analysis.

Remark 3.1.4. In the classical $p$-adic Hodge theory of $p$-adic Galois representations, this $\theta$ is nothing but the Sen operator; cf. Theorem 12 of [Sen81], Théorème 1.9 of [BC16]. So it is reasonable to call it relative Sen operator.

Remark 3.1.5. It is natural to ask whether there is a canonical representative of $\theta$ so that one can glue them in some global situation. First, it turns out that the natural place for $\theta$ to live is $B \otimes_{A} \Omega_{A / C}^{1}(S) \otimes_{\mathbb{Q}_{p}}$ $\operatorname{Lie}(G)(-1)$, where $\Omega_{A / C}^{1}(S)$ denotes the continuous 1-forms of $A$ over $C$ with simple poles at $S$ and (1) denotes the usual Tate-twist. So $\theta$ is viewed as a (log) Higgs field.

Secondly, if $S$ is empty - that is, $\widetilde{X}$ is a $G$-Galois pro-étale perfectoid covering of $X$ - then we can make this element canonical using the functorial isomorphism $\Omega_{A / C}^{1}(-1) \cong H_{\text {cont }}^{1}(G, B)$ (Proposition 3.23 of [Sch13b]). Here $H_{\text {cont }}^{1}(G, B)$ denotes the continuous group cohomology group and is identified with $H^{1}\left(X_{\text {proét }}, \hat{\mathcal{O}}_{X}\right)$ by an argument similar to the proof of Lemma 5.6 of [Sch13a]. By the functorial property, it is enough to pin down this element $\theta$ for a particular $\widetilde{X}$, provided that $\theta_{\tilde{X}} \neq 0$. Indeed, if $\widetilde{X}^{\prime}$ is a $H$-Galois pro-étale perfectoid covering of $X$, we can consider $\widetilde{X}^{\prime \prime}:=\widetilde{X} \times_{X} \widetilde{X}^{\prime}$, the fibre product in the category of pro-étale coverings of $X$. Then $\widetilde{X}^{\prime \prime}$ is a $(G \times H)$-Galois pro-étale perfectoid covering of $X$. Once $\theta_{\widetilde{X}}$ is determined, so is $\theta_{\widetilde{X}^{\prime \prime}}$ and hence $\theta_{\widetilde{X}^{\prime}}$ by the functorial property.

Suppose $G \cong \mathbb{Z}_{p}$. Then there is a canonical isomorphism

$$
\Omega_{A / C}^{1}(-1) \otimes_{\mathbb{Q}_{p}} \operatorname{Lie}(G) \stackrel{\sim}{\longrightarrow} H_{\text {cont }}^{1}(G, B) \otimes_{\mathbb{Z}_{p}} G \stackrel{\sim}{\longrightarrow} B_{G},
$$

where the first map is induced by the exponential map of $G$ and $B_{G}$ denotes the $G$-coinvariants of $B$. Under this isomorphism, the canonical $\theta$ is given by the image of $1 \in B$ in $B_{G}$. It can be checked that this is independent of the choice of such a $\mathbb{Z}_{p}$-covering.

The proof of Proposition 3.23 of [Sch13b] crucially uses the fact that when $X$ is defined over some finite extension of $\mathbb{Q}_{p}$, then there is the Faltings's extension (Corollary 6.14 of [Sch13a]) which produces this canonical isomorphism. Faltings's extension has also been generalised to the log case; cf. Corollary 2.4.5 of [DLLZ18]. Hence, it is conceivable that there is a canonical $\theta$ in general.

\section{Example 3.1.6.}

○ $X=\operatorname{Spa}\left(C\left\langle T^{ \pm 1}\right\rangle, \mathcal{O}_{C}\left\langle T^{ \pm 1}\right\rangle\right)$, the 1-dimensional torus;

。 $\widetilde{X}=\operatorname{Spa}\left(C\left\langle T^{ \pm \frac{1}{p^{\infty}}}\right\rangle, \mathcal{O}_{C}\left\langle T^{ \pm \frac{1}{p^{\infty}}}\right\rangle\right)$, the inverse limit of $\operatorname{Spa}\left(C\left\langle T^{ \pm \frac{1}{p^{m}}}\right\rangle, \mathcal{O}_{C}\left\langle T^{ \pm \frac{1}{p^{m}}}\right\rangle\right)$; 
$\circ G=\mathbb{Z}_{p}$ which acts by $k \cdot T^{\frac{1}{p^{m}}}=\zeta^{\frac{k}{p^{m}}} T^{\frac{1}{p^{m}}}, k \in \mathbb{Z}_{p}$ where $\left\{\zeta_{p^{m}}\right\}_{m}$ is a choice of compatible system of $p^{m}$ th roots of unity.

The locally analytic vectors in $C\left\langle T^{ \pm \frac{1}{p^{\infty}}}\right\rangle$ are just $\bigcup_{m} C\left\langle T^{ \pm \frac{1}{p^{m}}}\right\rangle$, the smooth vectors. This can be deduced easily from the existence of Tate's normalised trace in this situation; that is, there is a continuous left inverse of the inclusion $C\left\langle T^{ \pm \frac{1}{p^{m}}}\right\rangle \hookrightarrow C\left\langle T^{ \pm \frac{1}{p^{\infty}}}\right\rangle$. See Lemma 3.2.5 or Théorème 3.2 of [BC16]. The differential operator $\theta$ in this case is $\frac{d T}{T} \otimes \mathbf{1}$, where $\mathbf{1}$ is the image of $1 \in \mathbb{Z}_{p} \stackrel{\log }{\longrightarrow} \operatorname{Lie}\left(\mathbb{Z}_{p}\right)$ under the logarithm. The same result holds for $X=\operatorname{Spa}\left(C\langle T\rangle, \mathcal{O}_{C}\langle T\rangle\right)$, the 1-dimensional unit ball and $\widetilde{X}=\operatorname{Spa}\left(C\left\langle T^{\frac{1}{p^{\infty}}}\right\rangle, \mathcal{O}_{C}\left\langle T^{\frac{1}{p^{\infty}}}\right\rangle\right)$.

Remark 3.1.7. As mentioned in Remark 3.1.5, $\theta$ should be considered as a log Higgs field. This is closely related to previous work of Faltings [Fal05], Abbes-Gros-Tsuji [AGT16] and Liu-Zhu [LZ17] on $p$-adic Simpson correspondences. More precisely, for a finite-dimensional continuous representation $V$ of $G$, the $p$-adic Simpson correspondence associates a log Higgs field (after extending the coefficients to $B$ ):

$$
\phi_{V}: B \otimes_{\mathbb{Q}_{p}} V \rightarrow \Omega_{A / C}^{1}(S) \otimes_{A} B \otimes_{\mathbb{Q}_{p}} V(-1),
$$

which is monoidal in $V$. Hence, from the Tannakian point of view, this gives rise to a log Higgs field which is nothing but our $\theta$. Basically, we will construct $\theta$ by taking $V$ as the space of analytic functions on $G$ (after taking a certain limit).

To see that $\theta$ annihilates $B^{\text {la }}$, we remark that, as a $B$-module, $\operatorname{ker}\left(\phi_{V}\right)$ are generated by the $G$-smooth vectors in $B \otimes_{\mathbb{Q}_{p}} V$. Hence, when $V$ is the space of analytic functions on $G$, it is tautological that $B^{\text {la }}$ is in the kernel of $\theta$. I hope this provides some intuition for the constructions below.

\subsection{Relative Sen theory}

In this subsection, we will first define a bigger perfectoid algebra $B_{\infty}$ containing $B$ and construct (functorially) an operator in the endomorphism group $\operatorname{End}_{B_{\infty}}\left(B_{\infty} \otimes_{\mathbb{Q}_{p}} V\right)$ for any continuous finitedimensional $\mathbb{Q}_{p}$-representation $V$ of $G$.

3.2.1. Fix an étale map $f_{1}: X \rightarrow Y$ which factors as a composite of rational embeddings and finite étale maps, where $Y$ is either $\mathbb{T}^{1}=\operatorname{Spa}\left(C\left\langle T^{ \pm 1}\right\rangle, \mathcal{O}_{C}\left\langle T^{ \pm 1}\right\rangle\right)$ or $\mathbb{B}^{1}=\operatorname{Spa}\left(C\langle T\rangle, \mathcal{O}_{C}\langle T\rangle\right)$. In the latter case, we assume the image of $S$ in $Y$ is 0 . We will fix such a choice of $f_{1}: X \rightarrow Y$ from now on.

For any $n \in \mathbb{N}$, when $Y=\mathbb{T}^{1}\left(\right.$ respectively $\left.\mathbb{B}^{1}\right)$, let

$$
\begin{aligned}
& Y_{n}=\operatorname{Spa}\left(R_{n}, R_{n}^{+}\right):=\operatorname{Spa}\left(C\left\langle T^{ \pm \frac{1}{p^{n}}}\right\rangle, \mathcal{O}_{C}\left\langle T^{ \pm \frac{1}{p^{n}}}\right\rangle\right)\left(\text { respectively } \operatorname{Spa}\left(C\left\langle T^{\frac{1}{p^{n}}}\right\rangle, \mathcal{O}_{C}\left\langle T^{\frac{1}{p^{n}}}\right\rangle\right)\right), \\
& Y_{\infty}=\operatorname{Spa}\left(R, R^{+}\right):=\operatorname{Spa}\left(C\left\langle T^{ \pm \frac{1}{p^{\infty}}}\right\rangle, \mathcal{O}_{C}\left\langle T^{ \pm \frac{1}{p^{\infty}}}\right\rangle\right)\left(\text { respectively } \operatorname{Spa}\left(C\left\langle T^{\frac{1}{p^{\infty}}}\right\rangle, \mathcal{O}_{C}\left\langle T^{\frac{1}{p^{\infty}}}\right\rangle\right)\right) .
\end{aligned}
$$

This is the example considered in Example 3.1.6. Note that $Y_{\infty} \sim \lim _{i \in \mathbb{N}} Y_{i}$ is a Galois covering of $Y$ and $R^{+}$is the $p$-adic completion of $\lim _{n} R_{n}^{+}$. We denote the Galois group by $\Gamma$, which can be identified with $\mathbb{Z}_{p}$ noncanonically. For $n \geq m$, there is the usual trace map

$$
\operatorname{tr}_{Y, n, m}: R_{n}^{+} \rightarrow R_{m}^{+}
$$

Concretely, it sends $T^{\frac{l}{p^{k}}},(l, p)=1$ to $p^{n-m} T^{\frac{l}{p^{k}}}$ if $k \leq m$ and 0 otherwise. Clearly, $\frac{1}{p^{n-m}} \operatorname{tr}_{Y, n, m}$ are compatible when $n$ varies and extends to a $R_{m}^{+}$-linear map

$$
{\overrightarrow{\operatorname{tr}_{Y, m}}}^{+}: R^{+} \rightarrow R_{m}^{+}
$$


which is a left inverse of the inclusion $R_{m}^{+} \rightarrow R^{+}$. It commutes with the action of $\Gamma$ as taking traces commutes with the Galois action. Moreover, for any $x \in R^{+}$,

$$
\lim _{m \rightarrow+\infty} \overline{\operatorname{tr}}_{Y, m}^{+}(x)=x \text {. }
$$

After inverting $p$, we get a $R_{m}$-linear map $\overline{\operatorname{tr}}_{Y, m}: R \rightarrow R_{m}$ (Tate's normalised trace). Let $\gamma$ be any topological generator of $p^{m} \Gamma$. It is easy to see that $\gamma-1$ is invertible on $\operatorname{ker}\left(\overline{\operatorname{tr}}_{Y, m}\right)$. Moreover, the norm $\left\|(\gamma-1)^{-1}\right\|$ on $\operatorname{ker}\left(\overline{\operatorname{tr}}_{Y, m}\right)$ equals $\left|\left(\zeta_{p^{m+1}}-1\right)^{-1}\right|_{p}=p^{\frac{1}{p^{m}(p-1)}}$, which converges to 1 as $m \rightarrow \infty$.

3.2.2. The material here should be a consequence of some general results of Diao-Lan-Liu-Zhu on $\log$ affinoid perfectoid spaces. See 5.3 of [DLLZ19]. For our later applications, we give a more explicit presentation.

Now we base change everything along the map $X \rightarrow Y$ :

$$
\begin{gathered}
X_{n}=\operatorname{Spa}\left(A_{n}, A_{n}^{+}\right):=X \times_{Y} Y_{n}, \\
X_{\infty}=\operatorname{Spa}\left(A_{\infty}, A_{\infty}^{+}\right):=X \times_{Y} Y_{\infty},
\end{gathered}
$$

where all of the fibre products are taken in the category of adic spaces over $C$. We remark that $X \rightarrow Y$ is locally of finite type in the sense of [Hub96, Definition 1.2.1] and the fibre product exists by [Hub96, Proposition 1.2.2]. All of the fibre products we consider below will exist for the same reason.

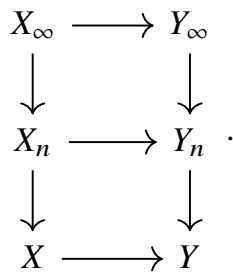

Recall that $C$ is equipped with an non-Archimedean norm $|\cdot|$. For any $|p|^{c} \in\left|C^{\times}\right| \subset \mathbb{R}_{\geq 0}^{\times}$, we fix a choice of element in $C$, formally written as $p^{c}$, with norm $|p|^{c}$.

Lemma 3.2.3. Let $\left(A_{n}^{+} \otimes_{R_{n}^{+}} R^{+}\right)^{\mathrm{tf}, \wedge}$ be the p-adic completion of the p-torsion free quotient of $A_{n}^{+} \otimes_{R_{n}^{+}} R^{+}$.

(1) The natural map $\left(A_{n}^{+} \otimes_{R_{n}^{+}} R^{+}\right)^{\mathrm{tf}, \wedge} \rightarrow A_{\infty}^{+}$is injective and its cokernel is killed by $p^{C_{n}}$ for some constant $C_{n}$ with $C_{n} \rightarrow 0$ as $n \rightarrow 0$.

(2) $A_{\infty}^{+}$is the p-adic completion of $\lim _{\longrightarrow} A_{n}^{+}$.

(3) $\left(A_{\infty}, A_{\infty}^{+}\right)$is a perfectoid affinoid $\left(C, \mathcal{O}_{C}\right)$-algebra.

Proof. As $X \rightarrow Y$ can be written as a composite of rational embeddings and finite étale maps, we may apply Lemma 4.5 of [Sch13a] here. Strictly speaking, Lemma 4.5 assumes $Y_{n}$ is étale over $Y$, but the same argument works here using that $R$ is a perfectoid $C$-algebra and $R^{+}$is the $p$-adic completion of $\underset{\lim _{n}}{\longrightarrow} R_{n}^{+}$

Hence, we may extend ( $A_{m}$-linearly) Tate's normalised trace $\overline{\operatorname{tr}}_{Y, m}$ to $X_{\infty}$ :

$$
\overline{\operatorname{tr}}_{X, m}: A_{\infty} \rightarrow A_{m}
$$

which is a continuous $A_{m}$-linear left inverse of the inclusion $A_{m} \rightarrow A_{\infty}$. Moreover, the image of $A_{\infty}^{+}$is contained in $p^{-C_{m}} A_{m}^{+}$for some constant $C_{m}$ with $C_{m} \rightarrow 0$ as $m \rightarrow 0$. For any $x \in A_{\infty}$, we still have

$$
\lim _{m \rightarrow+\infty} \overline{\operatorname{tr}}_{X, m}(x)=x \text {. }
$$

Note that $\Gamma$ acts on $X_{\infty}$ and commutes with $\overline{\operatorname{tr}}_{X, m}$. For any topological generator $\gamma$ of $p^{m} \Gamma$, the action of $\gamma-1$ on $\operatorname{ker}\left(\overline{\operatorname{tr}}_{X, m}\right)$ is invertible. The norm of its inverse $\left\|(\gamma-1)^{-1}\right\|$ is bounded by some $p^{c_{m}}$ for some constant $c_{m}>0$, and $c_{m} \rightarrow 0$ as $m \rightarrow \infty$. 
Remark 3.2.4. These properties of Tate's normalised trace appear as (TS2), (TS3) in the Tate-Sen conditions formulated by Berger-Colmez; cf. [BC08] Définition 3.1.3.

$A_{\infty}$ is a Banach space representation of $\Gamma$. We can consider its $\Gamma$-locally analytic vectors; cf. 2.1.4. The following lemma is a direct consequence of the existence of Tate's normalised traces. See also Théorème 3.2 of [BC16].

Lemma 3.2.5. $\left(A_{\infty}\right)^{p^{n} \Gamma-\mathrm{an}}=A_{n}$ for any integer $n \geq 0$. In particular, the subspace of $\Gamma$-locally analytic vectors in $A_{\infty}$ is $\bigcup_{n \geq 0} A_{n}$.

Proof. For $m \geq n$, as $\overline{\operatorname{tr}}_{X, m}$ is continuous and $\Gamma$-equivariant, it can be restricted to the $p^{n} \Gamma$-analytic vectors

$$
\left(A_{\infty}\right)^{p^{n} \Gamma-\mathrm{an}} \stackrel{\overline{\operatorname{tr}}_{X, m}}{\longrightarrow}\left(A_{m}\right)^{p^{n} \Gamma-\mathrm{an}}=\left(A_{m}\right)^{p^{n} \Gamma}=A_{n} .
$$

Note that the action of $p^{n} \Gamma$ on $A_{m}$ is trivial on $p^{m} \Gamma$; hence, the analyticity implies the first equality. Now the lemma follows from $\lim _{m \rightarrow+\infty} \overline{\operatorname{tr}}_{X, m}(x)=x$.

3.2.6. Next we base change the tower $\left\{X_{n}\right\}_{n}$ to $\widetilde{X}$. Suppose $G_{0}$ is an open subgroup of $G$. Let

$$
X_{G_{0}}:=\operatorname{Spa}\left(B^{G_{0}},\left(B^{+}\right)^{G_{0}}\right)
$$

be the corresponding covering of $X$. We can take the fibre product in the category of adic spaces over $C$

$$
X_{G_{0}, n}^{\prime}:=X_{G_{0}} \times_{X} X_{n}=X_{G_{0}} \times_{Y} Y_{n},
$$

and take its normalisation

$$
X_{G_{0}, n}=\operatorname{Spa}\left(B_{G_{0}, n}, B_{G_{0}, n}^{+}\right) .
$$

Note that by Abhyankar's lemma for rigid analytic spaces (cf. Lemma 4.2.2, 4.2.3 of [DLLZ19], which is based on earlier work of Lütkebohmert), $X_{G_{0}, n} \rightarrow Y_{n}$ is unramified when $n$ is sufficiently large; hence, $X_{G_{0}, n} \rightarrow X_{n}$ is finite étale and

$$
X_{G_{0}, n}=X_{G_{0}, m} \times_{X_{m}} X_{n}=X_{G_{0}, m} \times_{Y_{m}} Y_{n}
$$

for sufficiently large $m<n$. This implies that when $n$ is sufficiently large,

$$
X_{G_{0}, \infty}=\operatorname{Spa}\left(B_{G_{0}, \infty}, B_{G_{0}, \infty}^{+}\right):=X_{G_{0}, n} \times_{X_{n}} X_{\infty}=X_{G_{0}, n} \times_{Y_{n}} Y_{\infty}
$$

is independent of $n$. Since $X_{G_{0}, n} \rightarrow Y_{n}$ can be written as a composite of rational embeddings and finite étale maps, Lemma 3.2.3 still holds in this setting. For example, $B_{G_{0}, \infty}^{+}$is the $p$-adic completion of $\lim _{\longrightarrow} B_{G_{0}, n}^{+}$. For sufficiently large $n$, there exist Tate's normalised traces $\overline{\operatorname{tr}}_{X_{G_{0}}, n}: B_{G_{0}, \infty} \rightarrow B_{G_{0}, n}$ with same properties as in the case $\left\{X_{n}\right\}_{n}$. In particular, $\left(B_{G_{0}, \infty}\right)^{p^{n} \Gamma-\text { an }}=B_{G_{0}, n}$ for $n$ large enough.

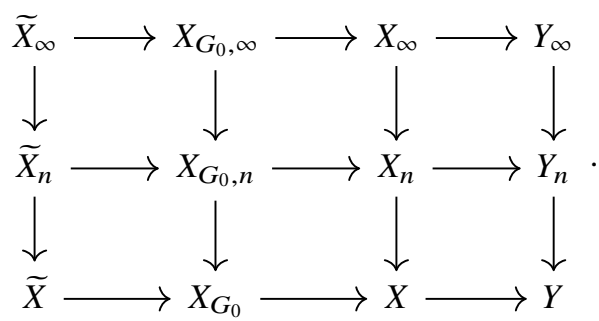

Suppose $G_{0}^{\prime}$ is another open subgroup of $G$ containing $G_{0}$. We have compatible maps $X_{G_{0}, n} \rightarrow X_{G_{0}^{\prime}, n}$ which are finite étale when $n$ is sufficiently large. Hence, $X_{G_{0}, \infty} \rightarrow X_{G_{0}^{\prime}, \infty}$ is a finite étale covering of affinoid perfectoid spaces. Let $B_{\infty}^{+}$be the $p$-adic completion of $\lim _{G_{0}} B_{G_{0}, \infty}^{+}$over all open subgroups 
$G_{0}$ of $G$ and $B_{\infty}=B_{\infty}^{+}\left[\frac{1}{p}\right]$ equipped with the norm induced from $B_{G_{0}, \infty}^{+}$. Then $\left(B_{\infty}, B_{\infty}^{+}\right)$is again an affinoid perfectoid $\left(C, \mathcal{O}_{C}\right)$-algebra as it is the completion of a direct limit of perfectoid affinoid $\left(C, \mathcal{O}_{C}\right)$-algebras. We denote $\operatorname{Spa}\left(B_{\infty}, B_{\infty}^{+}\right)$by $\widetilde{X}_{\infty}$. Note that $\widetilde{X}_{\infty}$ also agrees with the fibre product of $\tilde{X}=\lim _{\longleftarrow} X_{G_{0}}$ and $X_{\infty}=\lim X_{n}$ in the pro-Kummer étale site of $X$ equipped with the natural $\log$ structure defined by $S$; cf. [DLLZ $\overleftarrow{18}$ ].

Clearly, $G \times \Gamma$ acts on $B_{\infty}^{+}$and $B_{\infty}$. Then it follows from Faltings's almost purity theorem (cf. [Sch12, Theorem 7.9 (iii)]) that $\left(B_{\infty}^{+} /(p)\right)^{G_{0}}$ almost equals $B_{G_{0}, \infty}^{+} /(p)$. Hence, $\left(B_{\infty}\right)^{G_{0}}=B_{G_{0}, \infty}$ and $\left(B_{\infty}^{+}\right)^{G_{0}}$ almost equals $B_{G_{0}, \infty}^{+}$.

Proposition 3.2.7. $B_{\infty}$ satisfies the Tate-Sen conditions formulated by Berger-Colmez in [BC08, Définition 3.1.3] with

○ $S=\mathbb{Q}_{p}$;

- $\tilde{\Lambda}=B_{\infty}$ equipped with the valuation induced by its norm;

$\circ G_{0}=G \times \Gamma, H_{0}=G \times\{1\}$ as a subgroup of $G \times \Gamma$;

○ $\Lambda_{H, n}=B_{H, n}, R_{H, n}=\overline{\operatorname{tr}}_{X_{H}, n}$ for any open subgroup $H$ of $H_{0}=G$.

Proof. To see (TS1), for any open subgroups $H_{1} \subset H_{2}$ of $G$, we know that $\left(B_{\infty}\right)^{H_{2}}=B_{H_{2}, \infty} \rightarrow$ $\left(B_{\infty}\right)^{H_{1}}=B_{H_{1}, \infty}$ is a finite étale map between perfectoid algebras; hence, by Faltings's almost purity theorem, $B_{H_{2}, \infty}^{+} \rightarrow B_{H_{1}, \infty}^{+}$is almost finite étale. All of the claims in (TS2), (TS3) are essentially verified in 3.2.2 and the above discussion.

Remark 3.2.8. It follows from the definition directly that $B_{\infty}$ satisfies the Tate-Sen conditions with respect to the action of $G_{0} \times \Gamma$ for any open subgroup $G_{0}$ of $G$.

Lemma 3.2.9. $\left(B_{\infty}\right)^{\Gamma}=B$.

Proof. Recall that $\widetilde{X}=\operatorname{Spa}\left(B, B^{+}\right)$is perfectoid and $B^{+}$is the $p$-adic completion of the direct limit $\lim _{\longrightarrow G_{0}} B_{G_{0}, 0}^{+}$over all open subgroups $G_{0}$ of $G$. Let $n$ be a positive integer. Note that by our assumption on the ramification-index in Section 3.1.1 and Abhyankar's lemma (cf. Lemma 4.2.2, 4.2.3 of [DLLZ19]), $X_{G_{0}, n} \rightarrow X_{G_{0}, 0}$ is finite étale for sufficiently small $G_{0}$. Hence, for a sufficiently small subgroup $G_{0}$, the fibre product

$$
\widetilde{X}_{n}=\operatorname{Spa}\left(B_{n}, B_{n}^{+}\right):=\widetilde{X} \times_{X_{G_{0}, 0}} X_{G_{0}, n}
$$

is independent of the choice of $G_{0}$ and $\widetilde{X}_{n} \rightarrow \widetilde{X}$ is a finite étale map between affinoid perfectoid spaces. By the same argument as in the proof of Lemma 3.2.3, $B_{n}^{+}$is the $p$-adic completion of $\lim _{G_{0}} B_{G_{0}, n}^{+}$. Hence, it follows from our discussion in Section 3.2.6 that $B_{\infty}^{+}$is the $p$-adic completion of $\lim _{n} B_{n}^{+}$. By Faltings's almost purity theorem, we see that $\left(B_{\infty}^{+}\right)^{\Gamma}$ is almost $B^{+}$and $\left(B_{\infty}\right)^{\Gamma}=B$.

3.2.10. Now suppose $V$ is a finite-dimensional continuous representation of $G$ over $\mathbb{Q}_{p}$. We are going to construct a morphism: $\operatorname{Lie}(\Gamma) \rightarrow \operatorname{End}_{B_{\infty}}\left(B_{\infty} \otimes_{\mathbb{Q}_{p}} V\right)$; that is, a Lie algebra representation of $\operatorname{Lie}(\Gamma)$ on $B_{\infty} \otimes_{\mathbb{Q}_{p}} V$.

As $G$ is compact, there exists a $G$-stable $\mathbb{Z}_{p}$-lattice $T \subset V$. Let $\left(B_{\infty}\right)^{\circ} \subset B_{\infty}$ be the subset of powerbounded elements (equivalently elements with spectral norm at most 1). Note that $\left(B_{\infty}\right)^{\circ} \otimes_{\mathbb{Z}_{p}} T$ carries a diagonal action of $G$ and an action of $\Gamma$ on the first factor. This defines an action of $G \times \Gamma$ on $\left(B_{\infty}\right)^{\circ} \otimes_{\mathbb{Z}_{p}} T$.

Proposition 3.2.11. Fix a constant $c<\frac{1}{2}$ inside $\left|C^{\times}\right| \subset \mathbb{R}_{\geq 0}^{\times}$. Suppose $G_{0}$ is an open subgroup of $G$ acting trivially on $T / p T$. Then there exists a constant $n\left(G_{0}\right)$, which is independent of $T$ and only depends on $c$, such that for any integer $n \geq n\left(G_{0}\right)$, the tensor product $\left(B_{\infty}\right)^{\circ} \otimes_{\mathbb{Z}_{p}}$ T has a unique free $B_{G_{0}, n}^{+}$-submodule $D_{G_{0}, n}^{+}(T)$ of rank $\operatorname{dim}_{\mathbb{Q}_{p}} V$ with the following properties:

(1) $D_{G_{0}, n}^{+}(T)$ is fixed by $G_{0}$ and $G \times \Gamma$-stable;

(2) the natural morphism $\left(B_{\infty}\right)^{\circ} \otimes_{B_{G_{0}, n}^{+}} D_{G_{0}, n}^{+}(T) \rightarrow\left(B_{\infty}\right)^{\circ} \otimes_{\mathbb{Z}_{p}} T$ is an isomorphism;

(3) there exists a basis $\mathfrak{B}$ of $D_{G_{0}, n}^{+}(T)$ over $B_{G_{0}, n}^{+}$such that $(\gamma-1)(\mathfrak{B}) \subset p^{c} D_{G_{0}, n}^{+}(T)$ for any $\gamma \in \Gamma$; 
(4) $(\gamma-1)^{m}\left(D_{G_{0}, n}^{+}(T)\right) \subset p D_{G_{0}, n}^{+}(T)$ for any $\gamma \in \Gamma$ and $m \geq m(c, n)$, a constant only depending on $c, n$.

Proof. This follows from Proposition 3.3.1 of [BC08] by choosing $c_{3}=c$ and $c_{1}, c_{2}$ sufficiently small such that $c_{1}+2 c_{2}+2 c_{3}<1$. Note that in our setup, we may choose the constants $c_{1}, c_{2}, c_{3}$ in the TateSen conditions to be arbitrarily small. The first three parts are the same as [BC08, 3.3.1]. The last part is a consequence of the third one.

Now we construct an action of $\operatorname{Lie}(\Gamma)$ on $B_{\infty} \otimes_{\mathbb{Q}_{p}} V$. Choose a constant $c$, a sufficiently small open subgroup $G_{0}$ and $n \geq n\left(G_{0}\right)$ as in the proposition. By Amice's result (see Example 2.1.9), the last part of the proposition implies that the action of $p^{m} \Gamma$ on $D_{G_{0}, n}^{+}(T) \otimes_{\mathbb{Z}_{p}} \mathbb{Q}_{p}$ is analytic for sufficiently large $m$. Thus, $\operatorname{Lie}(\Gamma)$ acts on $D_{G_{0}, n}^{+}(T) \otimes_{\mathbb{Z}_{p}} \mathbb{Q}_{p}$. Since $B_{G_{0}, n}$ is fixed by $p^{n} \Gamma$, this action of $\operatorname{Lie}(\Gamma)$ is $B_{G_{0}, n}$-linear. By the second part of the proposition, we may extend it $B_{\infty}$-linearly to an action of $\operatorname{Lie}(\Gamma)$ on $\left(B_{\infty}\right)^{\circ} \otimes_{\mathbb{Z}_{p}} T \otimes \mathbb{Q}_{p}=B_{\infty} \otimes_{\mathbb{Q}_{p}} V$. This action commutes with the action of $G \times \Gamma$ as the two actions commute when restricted to $D_{G_{0}, n}^{+}(T) \otimes \mathbb{Q}_{p}$.

Note that the second part of Proposition 3.2.11 implies that

$$
\left(B_{\infty} \otimes_{\mathbb{Q}_{p}} V\right)^{G_{0}}=\left(B_{\infty}\right)^{G_{0}} \otimes_{B_{G_{0}, n}^{+}} D_{G_{0}, n}^{+}(T)=B_{G_{0}, \infty} \otimes_{B_{G_{0}, n}^{+}} D_{G_{0}, n}^{+}(T) .
$$

Lemma 3.2.12. For any $m \geq m(c, n)$, the subspace of $p^{m} \Gamma$-analytic vectors in $\left(B_{\infty} \otimes V\right)^{G_{0}}$ is $B_{G_{0}, m} \otimes_{B_{G_{0}, n}^{+}} D_{G_{0}, n}^{+}(T)$. In particular, the second part of Proposition 3.2 .11 implies that there is a natural isomorphism

$$
\left(B_{\infty} \otimes_{\mathbb{Q}_{p}} V\right)^{G_{0}, p^{m} \Gamma-\mathrm{an}} \otimes_{B_{G_{0}, m}} B_{\infty} \cong B_{\infty} \otimes_{\mathbb{Q}_{p}} V .
$$

Proof. For any $k \geq m$, the normalised trace $\overline{\operatorname{tr}}_{X_{G_{0}}, k}: B_{G_{0}, \infty} \rightarrow B_{G_{0}, k}$ induces a map

$$
\left(B_{\infty} \otimes_{\mathbb{Q}_{p}} V\right)^{G_{0}, p^{m} \Gamma-\mathrm{an}}=\left(B_{G_{0}, \infty} \otimes_{B_{G_{0}, n}^{+}} D_{G_{0}, n}^{+}(T)\right)^{p^{m} \Gamma-\text { an }} \rightarrow\left(B_{G_{0}, k} \otimes_{B_{G_{0}, n}^{+}} D_{G_{0}, n}^{+}(T)\right)^{p^{m} \Gamma-\text { an }} .
$$

As the action of $p^{m} \Gamma$ on $B_{G_{0}, k}$ is trivial on $p^{k} \Gamma$, we have a natural decomposition

$$
B_{G_{0}, k}=\bigoplus_{\chi: p^{m} \Gamma / p^{k} \Gamma \rightarrow C^{\times}} B_{G_{0}, k}[\chi]
$$

into the direct sum of $\chi$-isotypic components, where $\chi$ runs through all characters of $p^{m} \Gamma / p^{k} \Gamma$. Note that $\chi$ is an analytic function on $p^{m} \Gamma$ only when $\chi$ is trivial. Hence,

$$
\left(B_{G_{0}, k} \otimes_{B_{G_{0}, n}^{+}} D_{G_{0}, n}^{+}(T)\right)^{p^{m} \Gamma-\mathrm{an}}=\left(B_{G_{0}, k}\right)^{p^{m} \Gamma} \otimes_{B_{G_{0}, n}^{+}} D_{G_{0}, n}^{+}(T)=B_{G_{0}, m} \otimes_{B_{G_{0}, n}^{+}} D_{G_{0}, n}^{+}(T) .
$$

The rest of the proof is the same as the one of Lemma 3.2.5.

If $G_{0}$ is moreover a normal subgroup of $G$, we may choose $n$ large enough so that $B_{G, n} \rightarrow B_{G_{0}, n}$ is finite étale. Then by Galois descent, there is a $\Gamma$-equivariant isomorphism

$$
\left(D_{G_{0}, n}^{+}(T) \otimes_{\mathbb{Z}_{p}} \mathbb{Q}_{p}\right)^{G} \otimes_{B_{G, n}} B_{G_{0}, n}=D_{G_{0}, n}^{+}(T) \otimes_{\mathbb{Z}_{p}} \mathbb{Q}_{p}
$$

Let $D_{G, n}(V)=\left(D_{G_{0}, n}^{+}(T) \otimes_{\mathbb{Z}_{p}} \mathbb{Q}_{p}\right)^{G}$. By the second part of Proposition 3.2.11, there is a natural isomorphism

$$
B_{\infty} \otimes_{B_{G, n}} D_{G, n}(V) \cong B_{\infty} \otimes_{\mathbb{Q}_{p}} V
$$

and one can repeat all of the above discussion with $G_{0}$ replaced by $G$. Hence, we may reformulate the above construction into the following form, which clearly is independent of the choice of $T, c, G_{0}, n$. 
Proposition 3.2.13. For each finite-dimensional continuous representation $V$ of $G$ over $\mathbb{Q}_{p}$, there exists a (necessarily unique) $B_{\infty}$-linear action of $\mathrm{Lie}(\Gamma)$ on $B_{\infty} \otimes_{\mathbb{Q}_{p}} V$

$$
\phi_{V}: \operatorname{Lie}(\Gamma) \rightarrow \operatorname{End}_{B_{\infty}}\left(B_{\infty} \otimes_{\mathbb{Q}_{p}} V\right)
$$

extending the natural action of $\operatorname{Lie}(\Gamma)$ on the $\Gamma$-locally analytic vectors in $\left(B_{\infty} \otimes_{\mathbb{Q}_{p}} V\right)^{G}$. Moreover, it satisfies the following properties:

(1) $\phi_{V}$ commutes with the action of $G \times \Gamma$;

(2) $\phi_{V}$ is functorial in $V$; that is, suppose $\psi: V \rightarrow W$ is a G-equivariant map between $G$-representations, then $\mathbf{1} \otimes \psi: B_{\infty} \otimes V \rightarrow B_{\infty} \otimes W$ intertwines $\phi_{V}$ and $\phi_{W}$.

(3) $\phi_{V}$ commutes with tensor products; that is, suppose $V_{1}, V_{2}$ are two finite-dimensional representations of $G$, then $\phi_{V_{1}} \otimes \mathbf{1}+\mathbf{1} \otimes \phi_{V_{2}}=\phi_{V_{1} \otimes_{p} V_{2}}$ on $\left(B_{\infty} \otimes_{\mathbb{Q}_{p}} V_{1}\right) \otimes_{B_{\infty}}\left(B_{\infty} \otimes_{\mathbb{Q}_{p}} V_{2}\right)=B_{\infty} \otimes_{\mathbb{Q}_{p}}\left(V_{1} \otimes_{\mathbb{Q}_{p}} V_{2}\right)$.

Proof. It is easy to check all these properties on the $\Gamma$-locally analytic vectors in $\left(B_{\infty} \otimes_{\mathbb{Q}_{p}} V\right)^{G}$. We omit the details here.

If we fix a generator of $\operatorname{Lie}(\Gamma)$, then this proposition becomes the form claimed in the beginning of this subsection.

Suppose $G_{0}$ is an open subgroup of $G$. As $B_{\infty}$ satisfies the Tate-Sen conditions with respect to $G_{0} \times \Gamma$ (see Remark 3.2.8), Proposition 3.2.13 still holds with all $G$ replaced by $G_{0}$. Thus, Proposition 3.2.13 can be generalised as follows.

Proposition 3.2.14. For each finite-dimensional continuous representation $V$ of $G$ over $\mathbb{Q}_{p}$, there exists a (necessarily unique) $B_{\infty}$-linear action of $\mathrm{Lie}(\Gamma)$ on $B_{\infty} \otimes_{\mathbb{Q}_{p}} V$, extending its natural action on the $G$-smooth, $\Gamma$-locally analytic vectors in $B_{\infty} \otimes_{\mathbb{Q}_{p}} V$. Here an element in $B_{\infty} \otimes_{\mathbb{Q}_{p}} V$ is called $G$-smooth if it is fixed by some open subgroup of $G$. Moreover, this action satisfies all three properties as in the previous proposition.

Hence, this action of $\operatorname{Lie}(\Gamma)$ on $B_{\infty} \otimes_{\mathbb{Q}_{p}} V$ only depends on the restriction of the representation to any open subgroup of $G$. As it commutes with $\Gamma$, it induces a $B$-linear action of $\operatorname{Lie}(\Gamma)$ on $\left(B_{\infty} \otimes_{\mathbb{Q}_{p}} V\right)^{\Gamma}=$ $B \otimes_{\mathbb{Q}_{p}} V$ by Lemma 3.2.9.

\subsection{Proof of the main result I: construction}

3.3.1. We will first show that the action in Proposition 3.2.13 factors through $B \otimes \operatorname{Lie}(G)$. As a byproduct, this will imply Theorem 3.1.2. The strategy is to apply Proposition 3.2.13 to $V=\mathscr{C}^{\text {an }}\left(G, \mathbb{Q}_{p}\right)$, the space of analytic functions on $G$. However, as this is an infinite-dimensional vector space over $\mathbb{Q}_{p}$, it requires a limiting argument plus some extra work.

Let $G_{0} \subset G$ be a compact open subgroup equipped with an integer-valued, saturated $p$-valuation as in Section 2.1.1. By Proposition 2.1.3, we may replace $G_{0}$ by a smaller subgroup and assume that there is a dense sub-algebra $\lim _{k \in \mathbb{N}} V_{k} \subset \mathscr{C}^{\text {an }}\left(G_{0}, \mathbb{Q}_{p}\right)$, where each $V_{k}$ is a finite-dimensional subspace of $\mathscr{C}^{\text {an }}\left(G_{0}, \mathbb{Q}_{p}\right)$ stable under both the left and right translation actions of $G_{0}$. We will always view $V_{k}$ as a representation of $G_{0}$ using the left translation action.

Let $\mathscr{C}^{\text {an }}\left(G_{0}, \mathbb{Q}_{p}\right)^{o}$ be the unit ball of $\mathscr{C}^{\text {an }}\left(G_{0}, \mathbb{Q}_{p}\right)$ with respect to the norm $\|\cdot\|_{G_{0}}$ (see Section 2.1.1 for the notation here). Then

$$
V_{k}^{o}:=V_{k} \cap \mathscr{C}^{\mathrm{an}}\left(G_{0}, \mathbb{Q}_{p}\right)^{o}
$$

is a $G_{0}$-stable lattice. Moreover, it follows from Lemma 2.1.2 that $V_{k}^{o} / p$ is fixed by an open subgroup $G_{1}$ of $G_{0}$ for all $k$. Now we apply Proposition 3.2.11 to $V_{k}$, with $G=G_{0}$ : fix a constant $c$ as in the proposition and $n \geq n\left(G_{1}\right)$. There is $D_{G_{1}, n}^{+}\left(V_{k}^{o}\right) \subset\left(B_{\infty}\right)^{o} \otimes V_{k}^{o}$ such that

$$
\left(B_{\infty}\right)^{\circ} \otimes_{B_{G_{1}, n}^{+}} D_{G_{1}, n}^{+}\left(V_{k}^{o}\right)=\left(B_{\infty}\right)^{\circ} \otimes_{\mathbb{Z}_{p}} V_{k}^{o} .
$$


By the uniqueness of $D_{G_{1}, n}^{+}\left(V_{k}^{o}\right)$, these $\left\{D_{G_{1}, n}^{+}\left(V_{k}^{o}\right)\right\}_{k}$ form a direct system. Hence, we may take the direct limit of this equality over $k$ :

$$
\left(B_{\infty}\right)^{\circ} \otimes_{B_{G_{1}, n}^{+}} \underset{k}{\lim } D_{G_{1}, n}^{+}\left(V_{k}^{o}\right)=\left(B_{\infty}\right)^{\circ} \otimes_{\mathbb{Z}_{p}} \underset{k}{\lim } V_{k}^{o} .
$$

By taking the $p$-adic completion and inverting $p$, we obtain

$$
B_{\infty} \widehat{\otimes}_{B_{G_{1}, n}} D_{G_{1}, n}=B_{\infty} \widehat{\otimes}_{\mathbb{Q}_{p}} \mathscr{C}^{\mathrm{an}}\left(G_{0}, \mathbb{Q}_{p}\right) .
$$

Here $D_{G_{1}, n}^{+}$is the $p$-adic completion of $\lim _{\longrightarrow} D_{G_{1}, n}^{+}\left(V_{k}^{o}\right)$ and $D_{G_{1}, n}=D_{G_{1}, n}^{+} \otimes_{\mathbb{Z}_{p}} \mathbb{Q}_{p}$ equipped with the $p$-adic topology. The right-hand side becomes $\mathscr{C}^{\text {an }}\left(G_{0}, \mathbb{Q}_{p}\right)$ as $\lim _{k} V_{k}$ is dense inside of it.

Now we can construct an action of $\operatorname{Lie}(\Gamma)$ as before: there is an integer $m \geq 0$ such that $(\gamma-$ $1)^{m} D_{G_{1}, n}^{+}\left(V_{k}^{o}\right) \subset p D_{G_{1}, n}^{+}\left(V_{k}^{o}\right)$ for all $k \geq 0$ and $\gamma \in \Gamma$. Hence,

$$
(\gamma-1)^{m} D_{G_{1}, n}^{+} \subset p D_{G_{1}, n}^{+}
$$

for any $\gamma \in \Gamma$. By Example 2.1.9, the action of $\Gamma$ on $D_{G_{1}, n}$ is locally analytic. Its Lie algebra action can be extended $B_{\infty}$-linearly to an action on $B_{\infty} \widehat{\otimes}_{B_{G_{1}, n}} D_{G_{1}, n}=B_{\infty} \widehat{\otimes}_{\mathbb{Q}_{p}} \mathscr{C}^{\text {an }}\left(G_{0}, \mathbb{Q}_{p}\right)$ :

$$
\phi_{G_{0}}: \operatorname{Lie}(\Gamma) \rightarrow \operatorname{End}_{B_{\infty}}\left(B_{\infty} \widehat{\otimes}_{\mathbb{Q}_{p}} \mathscr{C}^{\mathrm{an}}\left(G_{0}, \mathbb{Q}_{p}\right)\right)
$$

Again this action uniquely extends the natural action of $\operatorname{Lie}(\Gamma)$ on the $\Gamma$-locally analytic vectors in $\left(B_{\infty} \widehat{\otimes}_{\mathbb{Q}_{p}} \mathscr{C}^{\text {an }}\left(G_{0}, \mathbb{Q}_{p}\right)\right)^{G_{0}}$, where $G_{0}$ acts diagonally on $B_{\infty}$ and $\mathscr{C}^{\text {an }}\left(G_{0}, \mathbb{Q}_{p}\right)$. In fact, let $D_{G_{0}, n}^{+}=$ $\left(D_{G_{1}, n}^{+}\right)^{G_{0}}$ and $D_{G_{0}, n}=\left(D_{G_{1}, n}\right)^{G_{0}}=D_{G_{0}, n}^{+} \otimes_{\mathbb{Z}_{p}} \mathbb{Q}_{p}$. Then by arguments similar to Lemma 3.2.12 and the paragraph below it, we have the following lemma.

Lemma 3.3.2. For $n$ sufficiently large, $D_{G_{0}, n}$ is the subspace of $p^{n} \Gamma$-analytic vectors in $\left(B_{\infty} \widehat{\otimes}_{\mathbb{Q}_{p}} \mathscr{C}^{\mathrm{an}}\left(G_{0}, \mathbb{Q}_{p}\right)\right)^{G_{0}}=\left(B_{\infty}\right)^{G_{0}-\mathrm{an}}$, and $B_{\infty} \widehat{\otimes}_{B_{G_{0}, n}} D_{G_{0}, n}=B_{\infty} \widehat{\otimes}_{\mathbb{Q}_{p}} \mathscr{C}^{\text {an }}\left(G_{0}, \mathbb{Q}_{p}\right)$.

The action of $\operatorname{Lie}(\Gamma)$ via $\phi_{G_{0}}$ commutes with $\Gamma$ as both actions commute on $D_{G_{1}, n}$. Moreover, it follows from the functorial property of $\phi_{V_{k}}$ in Proposition 3.2.13 that the action of $\operatorname{Lie}(\Gamma)$ on $D_{G_{1}, n}^{+}\left(V_{k}^{o}\right) \otimes \mathbb{Q}_{p}$ commutes with the right translation action of $G_{0}$. By passing to the limit, we see that $\phi_{G_{0}}$ also commutes with the right translation action of $G_{0}$. Recall that the multiplication structure on $\mathscr{C}^{\text {an }}\left(G_{0}, \mathbb{Q}_{p}\right)$ induces maps $V_{k} \otimes V_{l} \rightarrow V_{k+l}$; cf. Proposition 2.1.3. Hence, the last part of Proposition 3.2.13 implies that for any $\theta \in \phi_{G_{0}}(\operatorname{Lie}(\Gamma)), f_{1}, f_{2} \in \mathscr{C}^{\text {an }}\left(G_{0}, \mathbb{Q}_{p}\right)$,

$$
\theta\left(f_{1}\right) f_{2}+f_{1} \theta\left(f_{2}\right)=\theta\left(f_{1} f_{2}\right)
$$

that is, $\theta$ is a derivation. Hence, we have proved the following.

Proposition 3.3.3. $\phi_{G_{0}}(x)$ acts as a $G_{0}$-right-invariant derivation on $B_{\infty} \widehat{\otimes}_{\mathbb{Q}_{p}} \mathscr{C}^{\text {an }}\left(G_{0}, \mathbb{Q}_{p}\right)$ for any $x \in \operatorname{Lie}(\Gamma)$. Moreover, it commutes with $\Gamma$.

Corollary 3.3.4. $\phi_{G_{0}}$ factors through $B \otimes_{\mathbb{Q}_{p}} \operatorname{Lie}\left(G_{0}\right) \subset \operatorname{End}_{B_{\infty}}\left(B_{\infty} \widehat{\otimes}_{\mathbb{Q}_{p}} \mathscr{C}^{\mathrm{an}}\left(G_{0}, \mathbb{Q}_{p}\right)\right)$. Here $\operatorname{Lie}\left(G_{0}\right)$ acts on $\mathscr{C}^{\text {an }}\left(G_{0}, \mathbb{Q}_{p}\right)$ by the infinitesimal action of the left translation of $G_{0}$, and we extend it $B_{\infty}$-linearly to an action of $B \otimes_{\mathbb{Q}_{p}} \operatorname{Lie}\left(G_{0}\right)$ on $B_{\infty} \widehat{\otimes}_{\mathbb{Q}_{p}} \mathscr{C}^{\text {an }}\left(G_{0}, \mathbb{Q}_{p}\right)$.

Proof. Clearly, $\phi_{G_{0}}$ factors through $B_{\infty} \otimes_{\mathbb{Q}_{p}} \operatorname{Lie}\left(G_{0}\right)$. As it commutes with $\Gamma$, it also factors through $\left(B_{\infty} \otimes_{\mathbb{Q}_{p}} \operatorname{Lie}\left(G_{0}\right)\right)^{\Gamma}=B \otimes_{\mathbb{Q}_{p}} \operatorname{Lie}\left(G_{0}\right)$ by Lemma 3.2.9.

By abuse of notation, we also denote by $\phi_{G_{0}}: \operatorname{Lie}(\Gamma) \rightarrow B \otimes_{\mathbb{Q}_{p}} \operatorname{Lie}\left(G_{0}\right)$. Suppose we replace $G_{0}$ by a smaller subgroup $G_{0}^{\prime}$ and consider the restriction of $\mathscr{C}^{\text {an }}\left(G_{0}, \mathbb{Q}_{p}\right) \rightarrow \mathscr{C}^{\text {an }}\left(G_{0}^{\prime}, \mathbb{Q}_{p}\right)$. It is easy to 
see that $\phi_{G_{0}}=\phi_{G_{0}^{\prime}}$ once we identify $\operatorname{Lie}\left(G_{0}\right)=\operatorname{Lie}(G)=\operatorname{Lie}\left(G_{0}^{\prime}\right)$. Hence, $\phi_{G_{0}}$ is independent of the choice of $G_{0}$ and we denote it by

$$
\phi_{\widetilde{X}}: \operatorname{Lie}(\Gamma) \rightarrow B \otimes_{\mathbb{Q}_{p}} \operatorname{Lie}(G) .
$$

Corollary 3.3.5. The image of $\phi_{\widetilde{X}}$ acts trivially on the G-locally analytic vectors in $B$.

Proof. Recall that (see Section 2.1.1)

$$
B^{G_{0}-\mathrm{an}}=\left(B \widehat{\otimes}_{\mathbb{Q}_{p}} \mathscr{C}^{\mathrm{an}}\left(G_{0}, \mathbb{Q}_{p}\right)\right)^{G_{0}}=\left(B_{\infty} \widehat{\otimes}_{\mathbb{Q}_{p}} \mathscr{C}^{\mathrm{an}}\left(G_{0}, \mathbb{Q}_{p}\right)\right)^{G_{0} \times \Gamma},
$$

where the second equality follows from Lemma 3.2.9. Hence, by our construction of $\phi_{G_{0}}$, the action of $\operatorname{Lie}(\Gamma)$ is trivial on $B^{G_{0}-\text { an }}$. An easy computation shows that this action is nothing but $\phi_{\widetilde{X}}$. Note that this argument works for all sufficiently small $G_{0}$, which clearly implies the claim in the corollary.

Now if we fix a generator of $\operatorname{Lie}(\Gamma)$ and denote by $\theta_{\widetilde{X}}$ its image in $B \otimes_{\mathbb{Q}_{p}} \operatorname{Lie}(G)$ under $\phi_{\widetilde{X}}$, then we obtain the form claimed in the first part of Theorem 3.1.2.

Our next result implies that $\phi_{\widetilde{X}}$ is universal. Let $V$ be a finite-dimensional representation of $G$ over $\mathbb{Q}_{p}$. The action of $G$ on $V$ is locally analytic; hence, there is a natural $B_{\infty}$-linear action of $B_{\infty} \otimes_{\mathbb{Q}_{p}} \operatorname{Lie}(G)$ on $B_{\infty} \otimes_{\mathbb{Q}_{p}} V$. Therefore, $\phi_{\widetilde{X}}$ gives an action of $\operatorname{Lie}(\Gamma)$ on $B_{\infty} \otimes_{\mathbb{Q}_{p}} V$. On the other hand, we defined another action of $\operatorname{Lie}(\Gamma)$ called $\phi_{V}$ in Proposition 3.2.13.

Corollary 3.3.6. $\phi_{V}$ agrees with $\phi_{\widetilde{X}}$ for any $V$.

Proof. Let $G_{0}$ be a sufficiently small open subgroup of $G$ as in the beginning of Section 3.3.1. Moreover, we may assume $V$ is a $G_{0}$-analytic representation. Let $V^{*}$ be the dual vector space of $V$. Then taking the matrix coefficients of $V$ induces a map

$$
\begin{gathered}
m_{V}: V \otimes_{\mathbb{Q}_{p}} V^{*} \rightarrow \mathscr{C}^{\mathrm{an}}\left(G_{0}, \mathbb{Q}_{p}\right), \\
m_{V}(v \otimes l)(g)=l\left(g^{-1} \cdot v\right), v \in V, l \in V^{*}, g \in G_{0} .
\end{gathered}
$$

This map is $G_{0}$-equivariant where $G_{0}$ acts only on the first factor of $V \otimes V^{*}$ and acts via the left translation on $\mathscr{C}^{\text {an }}\left(G_{0}, \mathbb{Q}_{p}\right)$. The induced map

$$
\mathbf{1}_{B_{\infty}} \otimes m_{V}: B_{\infty} \otimes_{\mathbb{Q}_{p}} V \otimes_{\mathbb{Q}_{p}} V^{*} \rightarrow B_{\infty} \widehat{\otimes}_{\mathbb{Q}_{p}} \mathscr{C}^{\text {an }}\left(G_{0}, \mathbb{Q}_{p}\right)
$$

intertwines $\phi_{V \otimes V^{*}}=\phi_{V} \otimes \mathbf{1}_{V^{*}}$ and $\phi_{G_{0}}=\phi_{\widetilde{X}}$. One can check this on the $G_{0}$-fixed, $\Gamma$-locally analytic vectors. Trivially, this map also intertwines $\phi_{\widetilde{X}} \otimes \mathbf{1}_{V^{*}}$ and $\phi_{\tilde{X}}$. Now for any nonzero $v \in V$, there exists $l \in V^{*}$ such that $m_{V}(v \otimes l) \neq 0$. From this, it is easy to see that $\phi_{V}$ has to factor through $\phi_{\widetilde{X}}$.

Remark 3.3.7. This corollary gives a 'practical way' to compute $\phi_{\widetilde{X}}$ : choose a faithful representation $V$ of an open subgroup of $G$. Then $\phi_{V}$ completely determines $\phi_{\widetilde{X}}$.

Remark 3.3.8. $B \otimes_{\mathbb{Q}_{p}} \operatorname{Lie}(G)$ acts naturally on the $G$-locally analytic vectors $\left(B \otimes_{\mathbb{Q}_{p}} V\right)^{\text {la }}$ of $B \otimes_{\mathbb{Q}_{p}} V$. Hence, $\phi_{\widetilde{X}}: \operatorname{Lie}(\Gamma) \rightarrow B \otimes_{\mathbb{Q}_{p}} \operatorname{Lie}(G)$ induces an action of $\operatorname{Lie}(\Gamma)$ on $\left(B \otimes_{\mathbb{Q}_{p}} V\right)^{\text {la }}$. Combining Corollaries 3.3.5 and 3.3.6, it is easy to see that this action is nothing but $\phi_{V}$.

Corollary 3.3.9. $\phi_{\widetilde{X}}$ is functorial in the pair $(B, G)$; that is, suppose that $H$ is a closed normal subgroup of $G$ such that $\widetilde{X}^{\prime}=\operatorname{Spa}\left(B^{H},\left(B^{+}\right)^{H}\right)$ is a 'log $G / H$-Galois pro-étale perfectoid covering' of $X$ as in 3.1.1, then $\phi_{\widetilde{X}^{\prime}}: \operatorname{Lie}(\Gamma) \rightarrow B^{H} \otimes_{\mathbb{Q}_{p}} \operatorname{Lie}(G / H) \subset B \otimes_{\mathbb{Q}_{p}} \operatorname{Lie}(G / H)$ can be identified with the composite

$$
\operatorname{Lie}(\Gamma) \stackrel{\phi_{\tilde{X}}}{\longrightarrow} B \otimes_{\mathbb{Q}_{p}} \operatorname{Lie}(G) \stackrel{\bmod B \otimes_{\mathbb{Q}_{p}} \operatorname{Lie}(H)}{\longrightarrow} B \otimes_{\mathbb{Q}_{p}} \operatorname{Lie}(G / H)
$$

Proof. It is enough to check that the formulation of $\phi_{V}$ in Proposition 3.2.13 is functorial in a similar sense. But this basically follows from the construction. We omit the details here. 
We mention the following results concerning the uniqueness of our differential equation. Note that $\widetilde{X}_{\infty}=\operatorname{Spa}\left(B_{\infty}, B_{\infty}^{+}\right)$is a ' $\log G \times \Gamma$-Galois pro-étale perfectoid covering' of $X$. Let $B_{\infty}^{\text {la }}$ be the $G \times \Gamma$ locally analytic vectors in $B_{\infty}$.

Proposition 3.3.10. If $D \in B_{\infty} \otimes_{\mathbb{Q}_{p}} \operatorname{Lie}(G)$ and $D(v)=0$ for any $v \in B_{\infty}^{\text {la }}$, then $D=0$.

Proof. We will freely use notation introduced in Section 3.3.1. Let $G_{0}$ be a compact open subgroup of $G$ considered there. By Lemma 3.3.2, we have an isomorphism for sufficiently large $n$

$$
B_{\infty} \widehat{\otimes}_{B_{G_{0}, n}}\left(B_{\infty}\right)^{G_{0}-\mathrm{an}, p^{n} \Gamma-\mathrm{an}}=B_{\infty} \widehat{\otimes}_{\mathbb{Q}_{p}} \mathscr{C}^{\text {an }}\left(G_{0}, \mathbb{Q}_{p}\right) .
$$

It follows from Section 2.1.4 that this is equivariant with respect to the following actions of $G_{0}$ : the natural action on $\left(B_{\infty}\right)^{G_{0}-\text { an, } p^{n} \Gamma-\text { an }}$, the right translation action on $\mathscr{C}^{\text {an }}\left(G_{0}, \mathbb{Q}_{p}\right)$ and trivial actions on both $B_{\infty}$. Using this action of $G_{0}$, we get $B_{\infty}$-linear actions of $B_{\infty} \otimes_{\mathbb{Q}_{p}} \operatorname{Lie}(G)$ on both sides. In particular, $D$ annihilates both sides. However, if $D \neq 0$, we can always find a $B_{\infty}$-valued analytic function on $G_{0}$ which is not annihilated by $D$. Contradiction. This proves our claim.

Corollary 3.3.11. Suppose $D \in B_{\infty} \otimes_{\mathbb{Q}_{p}} \operatorname{Lie}(G \times \Gamma)$ annihilates $B_{\infty}^{\mathrm{la}}$. Then $D$ can be written as

$$
D=b d
$$

where $b \in B_{\infty}$ and $d \in \phi_{\widetilde{X}_{\infty}}(\operatorname{Lie}(\Gamma))$.

Proof. We note that by the functorial property Corollary 3.3.9, $\phi_{\widetilde{X}_{\infty}} \equiv \phi_{X_{\infty}} \bmod B_{\infty} \otimes_{\mathbb{Q}_{p}} \operatorname{Lie}(G)$; hence, the composite of $\phi_{\widetilde{X}_{\infty}}$ and the projection map

$$
\operatorname{Lie}(\Gamma) \stackrel{\phi_{\tilde{X}_{\infty}}}{\longrightarrow} B_{\infty} \otimes_{\mathbb{Q}_{p}} \operatorname{Lie}(G \times \Gamma) \rightarrow B_{\infty} \otimes_{\mathbb{Q}_{p}} \operatorname{Lie}(\Gamma)
$$

is simply the identity map. In particular, we can find $b \in B_{\infty}$ and $d \in \phi_{\widetilde{X} \infty}(\operatorname{Lie}(\Gamma))$ such that $D-b d \in$ $B_{\infty} \otimes_{\mathbb{Q}_{p}} \operatorname{Lie}(G)$. The rest follows from Corollary 3.3.5 and Proposition 3.3.10.

\subsection{Proof of the main result II: uniqueness}

3.4.1. In the previous section, we proved the existence of $\theta$ in Theorem 3.1.2. Note that the construction depends on a choice of an étale map $f_{1}: X \rightarrow Y=\mathbb{T}^{1}$ or $\mathbb{B}^{1}$ in Section 3.2.1. In this section, we will show that in fact $\theta$ is well-defined up to $A^{\times}$. People who are interested in global applications can skip reading this part as this part will not play any role later.

3.4.2. Consider another étale map $f_{2}: X \rightarrow Y$ which factors as a composite of rational embeddings and finite étale maps. Let

$$
\widetilde{X}^{\prime}:=X \times_{f_{2}, Y} Y_{\infty}
$$

be the pullback of the profinite covering $Y_{\infty}$ along $f_{2}$. See Section 3.2.1 for the definition of $Y_{\infty}$. In the notation below, we put a superscript ' for everything constructed using $f_{2}$ instead of $f_{1}$. For example, $\widetilde{X}^{\prime}=\operatorname{Spa}\left(B^{\prime}, B^{\prime+}\right)$ is an affinoid ' $\log G^{\prime}$-Galois pro-étale perfectoid covering' of $X$ with $G^{\prime}=\Gamma$. We would like to compute $\phi_{\widetilde{X}^{\prime}}: \operatorname{Lie}(\Gamma) \rightarrow B^{\prime} \otimes_{\mathbb{Q}_{p}} \operatorname{Lie}\left(G^{\prime}\right)=B^{\prime} \otimes_{\mathbb{Q}_{p}} \operatorname{Lie}(\Gamma)$ first. In general, we will use the functorial property to reduce to this computation.

We will freely use the notation introduced in the previous subsections. In particular, $\widetilde{X}_{\infty}^{\prime}=$ $\operatorname{Spa}\left(B_{\infty}^{\prime}, B_{\infty}^{\prime+}\right)$ is a $G^{\prime} \times \Gamma$-covering of $X$. It is also a $G^{\prime}$-covering of $X_{\infty}=\operatorname{Spa}\left(A_{\infty}, A_{\infty}^{+}\right)$and a $\Gamma$ covering of $\widetilde{X}^{\prime}=\operatorname{Spa}\left(B^{\prime}, B^{\prime+}\right)$. Note that by Abhyankar's lemma, both coverings are profinite étale of perfectoid algebras. By the Hochschild-Serre spectral sequence, we have

$$
H_{\text {cont }}^{1}\left(G^{\prime} \times \Gamma, B_{\infty}^{\prime}\right) \cong H_{\text {cont }}^{1}\left(\Gamma,\left(B_{\infty}^{\prime}\right)^{G^{\prime} \times\{\mathbf{1}\}}\right)=H_{\text {cont }}^{1}\left(\Gamma, A_{\infty}\right) \cong H_{\text {cont }}^{1}(\Gamma, A) .
$$


The first isomorphism follows from $H_{\text {cont }}^{1}\left(G^{\prime} \times\{\mathbf{1}\}, B_{\infty}^{\prime}\right)=0$, which is a consequence of the almost purity theorem. The last isomorphism follows from the existence and properties of the Tate normalised trace $\overline{\operatorname{tr}}_{X, 0}: A_{\infty} \rightarrow A$. We denote by $\tau_{1}$ the composite isomorphism $H_{\text {cont }}^{1}\left(G^{\prime} \times \Gamma, B_{\infty}^{\prime}\right) \stackrel{\sim}{\rightarrow}$ $H_{\text {cont }}^{1}(\Gamma, A)$.

Symmetrically, we can get another isomorphism $\tau_{2}: H_{\text {cont }}^{1}\left(G^{\prime} \times \Gamma, B_{\infty}^{\prime}\right) \stackrel{\sim}{\rightarrow} H_{\text {cont }}^{1}(\Gamma, A)$ by

$$
H_{\text {cont }}^{1}\left(G^{\prime} \times \Gamma, B_{\infty}^{\prime}\right) \cong H_{\text {cont }}^{1}\left(G^{\prime},\left(B_{\infty}^{\prime}\right)^{\{1\} \times \Gamma}\right)=H_{\text {cont }}^{1}\left(G^{\prime}, B^{\prime}\right)=H_{\text {cont }}^{1}\left(\Gamma, B^{\prime}\right) \cong H_{\text {cont }}^{1}(\Gamma, A) .
$$

As $H_{\text {cont }}^{1}(\Gamma, A)$ is a free $A$-module of rank 1 , the composite $\tau_{1} \circ \tau_{2}^{-1} \in \operatorname{End}_{A}\left(H_{\text {cont }}^{1}(\Gamma, A)\right)=A$ is given by an element $a \in A^{\times}$.

Lemma 3.4.3. $\phi_{\widetilde{X}^{\prime}}: \operatorname{Lie}(\Gamma) \rightarrow B^{\prime} \otimes_{\mathbb{Q}_{p}} \operatorname{Lie}(\Gamma)$ is multiplication by $a$.

Proof. Fix a topological generator $\gamma$ of $\Gamma$. Unravelling all of the isomorphisms above, we can find an element $b \in B_{\infty}^{\prime}$ such that

$$
\begin{aligned}
& (\gamma, \mathbf{1}) \cdot b-b=-1 \\
& (\mathbf{1}, \gamma) \cdot b-b=a
\end{aligned}
$$

Here $(\gamma, \mathbf{1}),(\mathbf{1}, \gamma)$ are elements in $G^{\prime} \times \Gamma=\Gamma \times \Gamma$. In particular, both actions of $\Gamma$ on $b$ are analytic.

As pointed out in Remark 3.3.7, in order to compute $\phi_{\widetilde{X}^{\prime}}$, we choose a faithful representation of $G^{\prime}=\Gamma$ on $V=\mathbb{Q}_{p}^{\oplus 2}$ :

$$
\Gamma \rightarrow \mathrm{GL}_{2}\left(\mathbb{Q}_{p}\right), \gamma \mapsto\left(\begin{array}{ll}
1 & 1 \\
0 & 1
\end{array}\right)
$$

Then $B_{\infty}^{\prime} \otimes_{\mathbb{Q}_{p}} V=\left(B_{\infty}^{\prime}\right)^{\oplus 2}$ has a $G^{\prime}$-fixed basis over $B_{\infty}^{\prime}$ :

$$
e_{1}=(1,0), e_{2}=(b, 1) \text {. }
$$

Moreover, it is easy to see that the action of $\Gamma$ on this basis is (locally) analytic. Therefore, $\phi_{V}$ is obtained by $B_{\infty}^{\prime}$-linearly extending the action of $\operatorname{Lie}(\Gamma)$ on $e_{1}, e_{2}$. Let $t \in \operatorname{Lie}(\Gamma)$ be the logarithm of $\gamma$. Then $t \cdot e_{1}=0$ and it follows from Equation (3.4.2) that $t \cdot e_{2}=a e_{1}$. Hence,

$$
\begin{aligned}
\phi_{V}: \operatorname{Lie}(\Gamma) & \rightarrow B_{\infty}^{\prime} \otimes_{\mathbb{Q}_{p}} \mathfrak{g l}_{2}\left(\mathbb{Q}_{p}\right) \\
t & \mapsto\left(\begin{array}{ll}
0 & a \\
0 & 0
\end{array}\right) .
\end{aligned}
$$

Comparing this with the definition of $V$, we see that $\phi_{\widetilde{X}^{\prime}}$ is just multiplication by $a$.

3.4.4. Now we are ready to prove $\phi_{\widetilde{X}}$ is well-defined up to $A^{\times}$. We will keep using the notation introduced in Section 3.4.2. Suppose $\widetilde{X}$ is ' $\log G$-Galois pro-étale perfectoid covering' of $X$. Now using $f_{2}: X \rightarrow Y$ instead of $f_{1}: X \rightarrow Y$ in the setup Section 3.2.1 and redoing everything before, then rather than $\phi_{\tilde{X}}$, we get

$$
\phi_{\tilde{X}}^{\prime}: \operatorname{Lie}(\Gamma) \rightarrow B \otimes_{\mathbb{Q}_{p}} \operatorname{Lie}(G) .
$$

Proposition 3.4.5. $a \phi_{\widetilde{X}}^{\prime}(x)=\phi_{\widetilde{X}}(x)$, for any $x \in \operatorname{Lie}(\Gamma)$. In particular, $\phi_{\widetilde{X}}^{\prime}$ and $\phi_{\widetilde{X}}$ differ by a unit of $A$.

Proof. First note that the special case $\widetilde{X}=X_{\infty}=\operatorname{Spa}\left(A_{\infty}, A_{\infty}^{+}\right)$is essentially proved by the same computation as above (after switching the role of $f_{1}$ and $f_{2}$ ). In general, let $V$ be a finite-dimensional continuous representation of $G$ over $\mathbb{Q}_{p}$. It is enough to show that $a^{-1} \phi_{V}$ on $B_{\infty} \otimes_{\mathbb{Q}_{p}} V$ agrees with the 
action of $\operatorname{Lie}(\Gamma)$ induced from $\phi_{\widetilde{X}}^{\prime}$. Consider $\widetilde{X}_{\infty}:=\operatorname{Spa}\left(B_{\infty}, B_{\infty}^{+}\right)$. This is a 'log $G \times \Gamma$-Galois pro-étale perfectoid covering' of $X$. Then by the functorial property Corollary 3.3.9,

$$
\phi_{\widetilde{X}}^{\prime} \equiv \phi_{\widetilde{X}_{\infty}}^{\prime} \bmod B_{\infty} \otimes_{\mathbb{Q}_{p}} \operatorname{Lie}(\Gamma)
$$

Hence, the actions of $\operatorname{Lie}(\Gamma)$ on $B_{\infty} \otimes_{\mathbb{Q}_{p}} V$ induced from $\phi_{\widetilde{X}}^{\prime}$ and $\phi_{\widetilde{X}_{\infty}}^{\prime}$ are the same because $\Gamma$ acts trivially on $V$. So it suffices to compare $a^{-1} \phi_{V}$ and $\phi_{\widetilde{X}_{\infty}}^{\prime}$.

Since both actions are $B_{\infty}$-linear, it follows from Proposition 3.2.11 that we only need to compare two actions on the $\Gamma$-locally analytic vectors in $\left(B_{\infty} \otimes_{\mathbb{Q}_{p}} V\right)^{G}$. Now on this $G$-fixed subspace, $\phi_{V}$ acts by the natural Lie algebra action of $\operatorname{Lie}(\Gamma)$, while by Remark 3.3.8 and the functorial property Corollary 3.3.9,

$$
\phi_{\widetilde{X}_{\infty}}^{\prime} \equiv \phi_{X_{\infty}}^{\prime} \bmod B_{\infty} \otimes_{\mathbb{Q}_{p}} \operatorname{Lie}(G)
$$

the action on $\left(B_{\infty} \otimes_{\mathbb{Q}_{p}} V\right)^{G, \Gamma-\text { la }}$ induced by $\phi_{\widetilde{X}_{\infty}}^{\prime}$ is just $\phi_{X_{\infty}}^{\prime}$. Hence, the desired equality is a direct consequence of the special case $\widetilde{X}=X_{\infty}$.

Remark 3.4.6. One can also use Corollary 3.3 .11 to reduce the general case to the special case $\widetilde{X}=X_{\infty}$.

\subsection{Locally analytic covering}

3.5.1. The goal of this subsection is to give a sufficient condition for $\theta$ to be nonzero. We will continue using the notation introduced before. In particular, there is a fixed étale map $f_{1}: X \rightarrow Y$ as in Section 3.2.1. First, let's recall Faltings's extension in the rigid analytic variety setting; cf. Corollary 6.14 of [Sch13a], Corollary 2.4.5 of [DLLZ18]. However, as both references assume $X$ is defined over a discretely valued complete non-Archimedean extension of $\mathbb{Q}_{p}$, we give a rather ad hoc definition here which will be sufficient for our purpose.

Fix a generator $\gamma$ of $\Gamma$ from now on and consider the following unipotent representation of $\Gamma$ on $V=\mathbb{Q}_{p}^{\oplus 2}$ :

$$
\Gamma \rightarrow \mathrm{GL}_{2}\left(\mathbb{Q}_{p}\right): \gamma \mapsto\left(\begin{array}{ll}
1 & 1 \\
0 & 1
\end{array}\right) .
$$

Clearly, $V$ is an extension of the trivial representation by itself:

$$
0 \rightarrow \mathbb{Q}_{p} \rightarrow V \rightarrow \mathbb{Q}_{p} \rightarrow 0
$$

Tensor this exact sequence with $B_{\infty}$ over $\mathbb{Q}_{p}$ and take $\Gamma$-invariants with respect to the diagonal actions:

$$
0 \rightarrow B \rightarrow\left(B_{\infty} \otimes_{\mathbb{Q}_{p}} V\right)^{\Gamma} \rightarrow B \rightarrow 0 .
$$

Note that this $G$-equivariant sequence is exact by the almost purity theorem. It follows from the discussion in 3.4.2 that its extension class is independent of the choice of $f_{1}$ up to multiplication by a unit of $A$.

The norm on $B_{\infty}$ induces norms on $B_{\infty} \otimes V=\left(B_{\infty}\right)^{\oplus 2}$ and its subspaces, and (FE) is continuous with respect to this topology. One important property of $(\mathrm{FE})$ is that if we take the continuous $G$-cohomology, the following connecting homomorphism of the long exact sequence is an isomorphism:

$$
A=B^{G} \stackrel{\sim}{\rightarrow} H_{\text {cont }}^{1}(G, B) .
$$

This can be seen by identifying

$$
H_{\text {cont }}^{i}(G, B) \cong H_{\text {cont }}^{i}\left(G \times \Gamma, B_{\infty}\right) \cong H_{\text {cont }}^{i}\left(\Gamma, A_{\infty}\right) \cong H_{\text {cont }}^{i}(\Gamma, A),
$$


where the first two isomorphisms follow from the almost purity theorem and the last isomorphism is a consequence of the Tate's normalised trace. Then it is easy to compute the connecting homomorphism.

In fact, this holds for any open subgroup $G_{0}$ of $G$; that is, if we take the continuous $G_{0}$-cohomology of (FE), the connecting homomorphism $B^{G_{0}} \rightarrow H_{\text {cont }}^{1}\left(G_{0}, B\right)$ is an isomorphism. To see this, note that $\operatorname{Spa}\left(B, B^{+}\right)$is a ' $\log G_{0}$-Galois pro-étale perfectoid covering' of $\operatorname{Spa}\left(B^{G_{0}},\left(B^{+}\right){ }^{G_{0}}\right)$. Hence, $H_{\text {cont }}^{1}\left(G_{0}, B\right)$ is also a free rank $1 B^{G_{0}}$-module. So in view of what we have proved, the claim follows from the fact that the natural map $B^{G_{0}} \otimes_{A} H_{\text {cont }}^{1}(G, B) \rightarrow H_{\text {cont }}^{1}\left(G_{0}, B\right)$ is an isomorphism.

Remark 3.5.2. If $X$ is defined over a finite extension of $\mathbb{Q}_{p}$, then (FE) recovers (log) Faltings's extension on $\tilde{X}$ defined in [Sch13a, DLLZ18] by identifying the quotient $B$ with $B \otimes_{A} \Omega_{A / C}^{1}(S)$ sending $1 \in B$ to $f_{1}^{*}\left(\frac{d T}{T}\right)$, where $\Omega_{A / C}^{1}(S)$ denotes the continuous 1 -forms of $A$ over $C$ with a simple pole at $S$. See Proposition 2.3.15 and proof of Corollary 2.4.2 of [DLLZ18]. Here $\tilde{X}$ is viewed as an open in the pro-Kummer étale site of $X$ equipped with the natural log structure defined by $S$ (cf. Example 2.1.2 of [DLLZ18]).

\section{Proposition 3.5.3. The following conditions are equivalent:}

(1) (FE) remains exact after taking $G_{0}$-analytic vectors for some open subgroup $G_{0}$ of $G$ equipped with an integer-valued, saturated p-valuation as in Section 2.1.1.

(2) (FE) remains exact after taking G-locally analytic vectors of each term.

(3) There exists a G-locally analytic vector $z \in B_{\infty}$ such that $\gamma(z)=z-1$.

Proof. The first two parts are clearly equivalent with existence of a $G$-locally analytic vector $x \in$ $\left(B_{\infty} \otimes_{\mathbb{Q}_{p}} V\right)^{\Gamma}$ mapping to $1 \in B$ in (FE). Write $B_{\infty} \otimes_{\mathbb{Q}_{p}} V=\left(B_{\infty}\right)^{\oplus 2}$. Then $x=(z, 1)$ for some $z \in B_{\infty}$ which is $G$-locally analytic and satisfies $\gamma(z)=z-1$. This proves the equivalence between these three conditions.

Definition 3.5.4. We say $\widetilde{X}$ is a locally analytic covering of $X$ if one of the conditions in Proposition 3.5.3 holds.

Remark 3.5.5. An analysis similar to Section 3.4.2 shows that this definition is independent of the choice of $f_{1}: X \rightarrow Y$. There is another intrinsic definition: $\widetilde{X}$ is a locally analytic covering of $X$ if and only if the natural map $H_{\text {cont }}^{1}\left(G, B^{\text {la }}\right) \rightarrow H_{\text {cont }}^{1}(G, B)$ is an isomorphism. We sketch a proof here. It follows from Tate's normalised trace that $H_{\text {cont }}^{1}\left(\Gamma,\left(A_{\infty}\right)^{\text {la }}\right) \cong H^{1}\left(\Gamma, A_{\infty}\right)$. Note that $A_{\infty}=\left(B_{\infty}\right)^{G}$. We claim that there are natural isomorphisms

$$
H_{\text {cont }}^{1}\left(G \times \Gamma,\left(B_{\infty}\right)^{\mathrm{la}}\right) \cong H_{\text {cont }}^{1}\left(\Gamma,\left(A_{\infty}\right)^{\mathrm{la}}\right) \cong H_{\text {cont }}^{1}\left(\Gamma, A_{\infty}\right) \cong H_{\text {cont }}^{1}\left(G \times \Gamma, B_{\infty}\right) .
$$

The first and third isomorphisms follow from the Hochschild-Serre spectral sequence and $H_{\text {cont }}^{1}\left(G, B_{\infty}\right)=H_{\text {cont }}^{1}\left(G,\left(B_{\infty}\right)^{\text {la }}\right)=0$. The vanishing of $H_{\text {cont }}^{1}\left(G, B_{\infty}\right)$ is clear by the almost purity theorem. For the vanishing of $H_{\text {cont }}^{1}\left(G,\left(B_{\infty}\right)^{\text {la }}\right)$, using Lemma 3.3.2, it suffices to show that $\lim _{n} H^{1}\left(G_{n}, \mathscr{C}^{\text {an }}\left(G_{n}, \mathbb{Q}_{p}\right)\right)=0$, which is true. Now we apply the Hochschild-Serre spectral sequence to the subgroup $\{\mathbf{1}\} \times \Gamma \subset G \times \Gamma$ in the computation of $H_{\text {cont }}^{1}\left(G \times \Gamma,\left(B_{\infty}\right)^{\text {la }}\right)$ and $H_{\text {cont }}^{1}\left(G \times \Gamma, B_{\infty}\right)$. Since $H_{\text {cont }}^{1}\left(\Gamma, B_{\infty}\right)=0$, it is easy to see that $H_{\text {cont }}^{1}\left(G, B^{\text {la }}\right) \cong H_{\text {cont }}^{1}(G, B)$ is equivalent with

$$
H^{1}\left(\Gamma,\left(B_{\infty}\right)^{\mathrm{la}}\right)^{G}=0
$$

that is, the $\Gamma$-coinvariants of $\left(B_{\infty}\right)^{\text {la }}$ have no $G$-invariants. Note that $1 \in\left(B_{\infty}\right)^{\text {la }}$ is fixed by $G$. Hence, the vanishing of $H^{1}\left(\Gamma,\left(B_{\infty}\right)^{\text {la }}\right)^{G}$ certainly implies the existence of element $z$ in the third part of Proposition 3.5.3. On the other hand, if there exists such an element $z$, the argument in Section 3.6.7 will imply that $H^{1}\left(\Gamma,\left(B_{\infty}\right)^{\mathrm{la}}\right)=0$. This finishes the proof.

Proposition 3.5.6. $\phi_{\widetilde{X}}: \operatorname{Lie}(\Gamma) \rightarrow B \otimes_{\mathbb{Q}_{p}} \operatorname{Lie}(G)$ is nonzero if $\widetilde{X}$ is a locally analytic covering of $X$. 
Proof. We need results established in Section 3.3.1. Let $G_{0}$ an open subgroup as in Section 3.3.1. Suppose $\phi_{\widetilde{X}}=0$. It follows from our construction there and Lemma 3.3.2 that $\operatorname{Lie}(\Gamma)$ is acting trivially on the $\Gamma$-locally analytic vectors in $\left(B_{\infty}\right)^{G_{0}-\text { an }}$. Hence, there does not exist an element $z \in\left(B_{\infty}\right)^{G_{0}-\text { an }}$ such that $\gamma(z)=z-1$. As $G_{0}$ can be taken arbitrarily small, part (3) of Proposition 3.5.3 implies that $\widetilde{X}$ is not a locally analytic covering of $X$.

\subsection{Application: acyclicity of taking locally analytic vectors of $B$}

We keep the notation and setup from Section 3.1.1. Our main result of this subsection gives an equivalent condition for $B$ being $\mathfrak{Q} \mathfrak{A}$-acyclic. See Subsection 2.2 for more details about this notion.

Theorem 3.6.1. Suppose $\widetilde{X}=\operatorname{Spa}\left(B, B^{+}\right)$is a 'log G-Galois pro-étale perfectoid covering' of $X$ and $X$ is small. Then $R^{i} \mathfrak{Q A}(B)=0$ for all $i \geq 1$ if and only if $\widetilde{X}$ is a locally analytic covering of $X$ (see Definition 3.5.4). When this happens, $B$ is strongly $\mathfrak{Q} \mathfrak{A}$-acyclic.

3.6.2. One direction is clear. Suppose $R^{i} \mathfrak{L A}(B)=0, i \geq 1$. Take completed tensor products of (FE) with $\mathscr{C}^{\text {an }}\left(G_{n}, \mathbb{Q}_{p}\right)$ over $\mathbb{Q}_{p}$ and take the continuous $G_{n}$-cohomology

$$
0 \rightarrow B^{G_{n}-\mathrm{an}} \rightarrow\left(B_{\infty} \otimes_{\mathbb{Q}_{p}} V\right)^{\Gamma, G_{n}-\mathrm{an}} \rightarrow B^{G_{n}-\mathrm{an}} \rightarrow H_{\text {cont }}^{1}\left(G_{n}, \mathscr{C}^{\text {an }}\left(G_{n}, \mathbb{Q}_{p}\right) \widehat{\otimes}_{\mathbb{Q}_{p}} B\right) .
$$

Then by passing to the direct limit with $n \rightarrow \infty$, we see that the last term vanishes. Hence, (FE) remains exact when taking $G$-locally analytic vectors.

3.6.3. The proof of the other direction goes as follows: first $H_{\text {cont }}^{i}\left(G_{n}, B \widehat{\otimes}_{\mathbb{Q}_{p}} \mathscr{C}^{\text {an }}\left(G_{n}, \mathbb{Q}_{p}\right)\right)$ will be identified with a certain group cohomology of $\Gamma$ and then we construct explicit elements to kill these cohomology groups. In particular, it implies strongly $\mathfrak{Q A}$-acyclicity. We remark that this is a standard technique in the theory of $(\varphi, \Gamma)$-modules for studying Galois cohomology of $p$-adic Galois representations.

Keep the notation introduced in Section 3.2.1. Let $G_{0}$ be an open subgroup considered in Section 3.3.1. We can consider $H_{\text {cont }}^{i}\left(G_{0} \times \Gamma, B_{\infty} \widehat{\otimes}_{\mathbb{Q}_{p}} \mathscr{C}^{\text {an }}\left(G_{0}, \mathbb{Q}_{p}\right)\right)$. On the one hand, by the almost purity theorem, $H_{\text {cont }}^{i}\left(\Gamma, B_{\infty}^{+} \otimes_{\mathbb{Z}_{p}} \mathscr{C}^{\text {an }}\left(G_{0}, \mathbb{Q}_{p}\right)^{\circ} / p\right)$ almost vanishes for $i \geq 1$; hence,

$$
H_{\text {cont }}^{i}\left(\Gamma, B_{\infty} \widehat{\otimes}_{\mathbb{Q}_{p}} \mathscr{C}^{\mathrm{an}}\left(G_{0}, \mathbb{Q}_{p}\right)\right)=0 .
$$

Applying the Hochschild-Serre spectral sequence to $\{\mathbf{1}\} \times \Gamma \subset G_{0} \times \Gamma$ and using Lemma 3.2.9, we get

$$
H_{\text {cont }}^{i}\left(G_{0}, B \widehat{\otimes}_{\mathbb{Q}_{p}} \mathscr{C}^{\text {an }}\left(G_{0}, \mathbb{Q}_{p}\right)\right) \stackrel{\sim}{\rightarrow} H_{\text {cont }}^{i}\left(G_{0} \times \Gamma, B_{\infty} \widehat{\otimes}_{\mathbb{Q}_{p}} \mathscr{C}^{\text {an }}\left(G_{0}, \mathbb{Q}_{p}\right)\right) .
$$

On the other hand, if we apply the Hochschild-Serre spectral sequence to $G_{0} \times\{\mathbf{1}\} \subset G_{0} \times \Gamma$ and note that $\left(B_{\infty} \widehat{\otimes}_{\mathbb{Q}_{p}} \mathscr{C}^{\text {an }}\left(G_{0}, \mathbb{Q}_{p}\right)\right)^{G_{0}}=\left(B_{\infty}\right)^{G_{0}-\text { an }}$, we get

$$
H_{\text {cont }}^{i}\left(\Gamma,\left(B_{\infty}\right)^{G_{0}-\mathrm{an}}\right) \stackrel{\sim}{\rightarrow} H_{\text {cont }}^{i}\left(G_{0} \times \Gamma, B_{\infty} \widehat{\otimes}_{\mathbb{Q}_{p}} \mathscr{C}^{\mathrm{an}}\left(G_{0}, \mathbb{Q}_{p}\right)\right) .
$$

All of these isomorphism are functorial in $G_{0}$. Therefore,

Lemma 3.6.4. There is a natural isomorphism

$$
H_{\text {cont }}^{i}\left(G_{0}, B \widehat{\otimes}_{\mathbb{Q}_{p}} \mathscr{C}^{\text {an }}\left(G_{0}, \mathbb{Q}_{p}\right)\right) \cong H_{\text {cont }}^{i}\left(\Gamma,\left(B_{\infty}\right)^{G_{0}-\text { an }}\right)
$$

functorial in $G_{0}$.

3.6.5. From this, it is clear that $H_{\text {cont }}^{i}\left(G_{0}, B \widehat{\otimes}_{\mathbb{Q}_{p}} \mathscr{C}^{\text {an }}\left(G_{0}, \mathbb{Q}_{p}\right)\right)=0, i \geq 2$ as $\Gamma$ is 1 -dimensional. So we assume $i=1$ from now on. Our next step is to replace $\left(B_{\infty}\right)^{G_{0}-\text { an }}$ on the right-hand side by a smaller 
space. Let $D_{G_{0}, n}$ be the subspace of $p^{n} \Gamma$-analytic vectors in $\left(B_{\infty}\right)^{G_{0}-\text { an }}$; that is, $\left(B_{\infty}\right)^{G_{0} \times p^{n} \Gamma-\text { an }}$. It follows from Lemma 3.3.2 that for sufficiently large $n$,

$$
\left(B_{\infty}\right)^{G_{0}-\text { an }}=B_{G_{0}, \infty} \widehat{\otimes}_{B_{G_{0}, n}} D_{G_{0}, n} .
$$

Moreover, let $D_{G_{0}, n}^{+}=B_{\infty}^{+} \cap D_{G_{0}, n}$; then by our construction in Section 3.3.1,

$$
(\gamma-1)^{m}\left(D_{G_{0}, n}^{+}\right) \subset p D_{G_{0}, n}^{+}
$$

for some $m$ depending on $n$. Fix such $n$ and $m$ for the moment. Recall that from results in Section 3.2.6, for $k$ large enough, there is Tate's normalised trace $\overline{\operatorname{tr}}_{X_{G_{0}, k}}: B_{G_{0}, \infty} \rightarrow B_{G_{0}, k}$ and $\gamma-1$ is invertible on $\operatorname{ker}\left(\overline{\operatorname{tr}}_{X_{G_{0}, k}}\right)$ with the norm of its inverse $\left\|(\gamma-1)^{-1}\right\|<p^{\frac{1}{2 m}}$. We claim that $H_{\text {cont }}^{1}$ $\left(\Gamma, \operatorname{ker}\left(\overline{\operatorname{tr}}_{X_{G_{0}, k}}\right) \widehat{\otimes}_{B_{G_{0}, n}} D_{G_{0}, n}\right)=0$ for such $k$. Assuming this, as $D_{G_{0}, k}=B_{G_{n}, k} \otimes_{B_{G_{0}, n}} D_{G_{0}, n}$, we get

$$
H_{\text {cont }}^{1}\left(\Gamma,\left(B_{\infty}\right)^{G_{0}-\text { an }}\right)=H_{\text {cont }}^{1}\left(\Gamma, D_{G_{0}, k}\right)=H_{\text {cont }}^{1}\left(\Gamma,\left(B_{\infty}\right)^{\left(G_{0} \times p^{k} \Gamma\right)-\text { an }}\right)
$$

for $k$ sufficiently large. Therefore,

$$
R^{1} \mathfrak{Q A}(B)=\underset{n, k}{\lim } H_{\text {cont }}^{1}\left(\Gamma,\left(B_{\infty}\right)^{\left(G_{n} \times p^{k} \Gamma\right)-\mathrm{an}}\right) .
$$

The vanishing of $H_{\text {cont }}^{1}\left(\Gamma, \operatorname{ker}\left(\overline{\operatorname{tr}}_{X_{G_{0}, k}}\right) \widehat{\otimes}_{B_{G_{0}, n}} D_{G_{0}, n}\right)$ is a consequence of the following easy lemma. Lemma 3.6.6. For any $a \in \operatorname{ker}\left(\overline{\operatorname{tr}}_{X_{G_{0}, k}}\right)^{+}:=\operatorname{ker}\left(\overline{\operatorname{tr}}_{X_{G_{0}, k}}\right) \cap B_{\infty}^{+}$and $b \in D_{G_{0}, n}^{+}$, we have pa $\otimes b \in$ $(\gamma-1)\left(\operatorname{ker}\left(\overline{\operatorname{tr}}_{X_{G_{0}, k}}\right)^{+} \widehat{\otimes}_{B_{G_{0}, k}^{+}} D_{G_{0}, n}^{+}\right)$.

Proof. Let $c=(\gamma-1)^{-1}(p a) \in \operatorname{ker}\left(\overline{\operatorname{tr}}_{X_{G_{0}, k}}\right)^{+}$. Consider the series

$$
\sum_{l=0}^{+\infty}\left(\gamma^{-1}-1\right)^{-l}(c) \otimes(\gamma-1)^{l}(b)=\sum_{l=0}^{+\infty} \gamma^{l}(1-\gamma)^{-l}(c) \otimes(\gamma-1)^{l}(b)
$$

which by our assumption converges to an element $x \in \operatorname{ker}\left(\overline{\operatorname{tr}}_{X_{G_{0}, k}}\right)^{+} \widehat{\otimes}_{B_{G_{0}, k}^{+}} D_{G_{0}, n}^{+}$. A direct computation gives $(\gamma-1)(x)=p a \otimes b$. Indeed, let

$$
y_{l}=\left(\gamma^{-1}-1\right)^{-l}(c), z_{l}=(\gamma-1)^{l}(b) .
$$

Then $(\gamma-1)\left(y_{l}\right)=-\gamma\left(y_{l-1}\right),(\gamma-1)\left(z_{l}\right)=z_{l+1}$. Hence,

$$
(\gamma-1)\left(y_{l} \otimes z_{l}\right)=(\gamma-1)\left(y_{l}\right) \otimes z_{l}+\gamma\left(y_{l}\right) \otimes(\gamma-1)\left(z_{l}\right)=-\gamma\left(y_{l-1}\right) \otimes z_{l}+\gamma\left(y_{l}\right) \otimes z_{l+1} .
$$

When taking the summation of $l$ from 0 to $+\infty$, only $-\gamma\left(y_{-1}\right) \otimes z_{0}$ is not cancelled out. But this is just $(\gamma-1)(c) \otimes b=p a \otimes b$.

3.6.7. Now it suffices to show that for any element $x \in\left(B_{\infty}\right)^{\left(G_{0} \times p^{k} \Gamma\right)-\text { an }}$, we can find $n>0$ and $l \geq k$ such that

$$
x \in(\gamma-1)\left(\left(B_{\infty}\right)^{\left(G_{n} \times p^{l} \Gamma\right)-\mathrm{an}}\right) .
$$

Fix such $k, x$. Let $\phi_{\gamma} \in \operatorname{Lie}(\Gamma)$ be the logarithm of $\gamma$. Then by Lemma 2.1.8, there exists a constant $C>0$ such that

$$
\left\|\phi_{\gamma}(x)\right\|_{G_{0} \times p^{k} \Gamma} \leq C\|x\|_{G_{0} \times p^{k} \Gamma} .
$$

Recall that $\|\cdot\|_{G_{0} \times p^{k} \Gamma}$ is a norm on the $G_{0} \times p^{k} \Gamma$-analytic vectors introduced in Section 2.1.1.

Recall that we assume $\widetilde{X}$ is a locally analytic covering of $X$. Hence, by part (3) of Proposition 3.5.3, we may find an element $z \in\left(B_{\infty}\right)^{\text {la }}$ such that $\gamma(z)=z-1$. By our construction of $B_{\infty}$ in 
Section 3.2.6, $\bigcup_{n, l} B_{G_{n}, l}$ is dense in $B_{\infty}$. Hence, there exists an element $z_{0} \in B_{G_{n}, l}$ for some $n, l$ such that $z \in\left(B_{\infty}\right)^{\left(G_{n} \times p^{l} \Gamma\right)-\text { an }}$ and the norm of $z-z_{0}$ (as an element in $B_{\infty}$ ) satisfies

$$
\left\|z-z_{0}\right\| \leq \frac{1}{2 C p^{1 /(p-1)}}
$$

Enlarging $n, l$ if necessary, we may assume $\left\|z-z_{0}\right\|_{G_{n} \times p^{l} \Gamma}=\left\|z-z_{0}\right\|$ by Lemma 2.1.8. Now consider the series

$$
-\sum_{m=0}^{+\infty} \frac{\phi_{\gamma}^{(m)}(x)}{(m+1) !}\left(z-z_{0}\right)^{m+1} .
$$

It is easy to see that $\left\|\frac{\phi_{\gamma}^{(m)}(x)}{(m+1) !}\left(z-z_{0}\right)^{m+1}\right\|_{G_{n} \times p^{l} \Gamma} \leq \frac{\|x\|_{G_{0} \times p^{k} \Gamma}}{2^{m+1} C}$. So this series converges (with respect to $\left.\|\cdot\|_{G_{n} \times p^{l} \Gamma}\right)$ to an element $y \in\left(B_{\infty}\right)^{\left(G_{n} \times p^{l} \Gamma\right)-\text { an }}$. Note that $\phi_{\gamma}\left(z-z_{0}\right)=\phi_{\gamma}(z)=-1$. A direct computation shows

$$
\phi_{\gamma}(y)=x
$$

After replacing $l$ by a larger integer, we may assume $(\gamma-1)^{m}\left(D_{G_{n}, l}^{+}\right) \subset p D_{G_{n}, l}^{+}$for some $m>0$ by our construction in Section 3.3.1. Recall that $D_{G_{n}, l}^{+}=\left(B_{\infty}\right)^{\left(G_{n} \times p^{l} \Gamma\right)-\text { an }} \cap B_{\infty}^{+}$. From this and $\phi_{\gamma}(y)=x$, we conclude $x \in(\gamma-1)\left(\left(B_{\infty}\right)^{\left(G_{n} \times p^{l} \Gamma\right)-\text { an }}\right)$ by the following simple lemma.

Lemma 3.6.8. Let $M$ be a unitary $\mathbb{Q}_{p}$-Banach representation of $\Gamma$ and $M^{o}$ be its unit ball. Suppose $(\gamma-1)^{m} M^{o} \subset p M^{o}$ for some $m \geq 0$. Then $\phi_{\gamma}(M) \subset(\gamma-1)(M)$.

Proof. The argument here was pointed out by a referee and is much simpler than my previous argument. One only needs to observe that $\phi_{\gamma}$ is the logarithm of $\gamma$; hence,

$$
\phi_{\gamma}=(\gamma-1) \sum_{m=0}(-1)^{m} \frac{(\gamma-1)^{m}}{m+1}
$$

and the second series converges to an operator on $M$ by our assumption.

Remark 3.6.9. The argument of this subsection is basically the same as the proof of Théorème 3.4 of [BC16]. Note that in their setting, the assumption 'locally analytic' always holds by Lemme 3.6 of [BC16].

For later applications, it will be useful to remove the smallness assumption on $X$ in Theorem 3.6.1. Unfortunately, we have to make some assumption in order to do this. Let $\widetilde{X}=\operatorname{Spa}\left(B, B^{+}\right)$be a ' $\log G$ Galois pro-étale perfectoid covering' of $X=\operatorname{Spa}\left(A, A^{+}\right)$as in Section 3.1.1 except that we do not require $X$ to be small anymore. Denote by $\pi: \widetilde{X} \rightarrow X$ and $\widetilde{\mathcal{O}}=\pi_{*} \mathcal{O}_{\widetilde{X}}$. Then we can consider subsheaf $\widetilde{\mathcal{O}}^{\text {la }} \subset \widetilde{\mathcal{O}}$ of $G$-locally analytic sections and subsheaves $\widetilde{\mathcal{O}}^{n} \subset \widetilde{\mathcal{O}}$ of $G_{n}$-analytic sections. Clearly, $\lim _{n} \widetilde{\mathcal{O}}^{n}=\mathcal{O}^{\text {la }}$.

Corollary 3.6.10. Suppose $\widetilde{X}=\operatorname{Spa}\left(B, B^{+}\right)$is a 'log $G$-Galois pro-étale perfectoid covering' of $X=$ $\mathrm{Spa}\left(A, A^{+}\right)$and $X$ can be covered by small rational open subsets $X_{i}, i=1, \cdots, k$, whose preimage $\widetilde{X}_{i}$ in $\tilde{X}$ is a locally analytic covering of $X_{i}$. Then

(1) $R^{i} \mathfrak{Q A}(B)=H^{i}\left(X, \widetilde{\mathcal{O}}^{\mathrm{la}}\right)=\check{H}^{i}\left(X, \widetilde{\mathcal{O}}^{\text {la }}\right)$ for any $i$, where $\check{H}^{i}\left(X, \widetilde{\mathcal{O}}^{\text {la }}\right)$ denotes the $\check{C}$ ech cohomology. In particular, $B$ is $\mathfrak{Q} \mathfrak{A}$-acyclic if and only if

$$
\check{H}^{i}\left(X, \widetilde{\mathcal{O}}^{\mathrm{la}}\right)=0, i \geq 1 .
$$


(2) Write $\mathfrak{U}_{0}=\left\{X_{1}, \cdots, X_{k}\right\}$. For any sheaf $F$ on $X$, denote by $\check{H}^{i}\left(\mathfrak{U}_{0}, F\right)$ the Čech cohomology of $F$ with respect to the cover $\mathfrak{U}_{0}$. Suppose the direct system $\left\{\breve{H}^{i}\left(\mathfrak{U}_{0}, \widetilde{\mathcal{O}}^{n}\right)\right\}_{n}$ is essentially zero for any $i>0$. Then $B$ is strongly $\mathfrak{Q A}$-acyclic.

Proof. The argument here is a standard application of Čech cohomology. Let $\mathfrak{B}$ be the set of small rational open subsets of $X$ contained in some $X_{i}$. It is easy to see that $\mathfrak{B}$ is closed under finite intersections and forms a basis of open subsets of $X$. Moreover, for any $U \in \mathfrak{B}, \pi^{-1}(U)$ is a locally analytic covering of $U$. We claim that for any $i \geq 1, U \in \mathfrak{B}$, the Čech cohomology

$$
\check{H}^{i}\left(U, \widetilde{\mathcal{O}}^{\mathrm{la}}\right)=0 .
$$

To see this, since $U$ is quasi-compact, any open cover of $U$ can be refined to a finite cover $\mathfrak{U} \subset \mathfrak{B}$. Let $C^{\bullet}(\mathfrak{H}, \widetilde{\mathcal{O}}), C^{\bullet}\left(\mathfrak{H}, \widetilde{\mathcal{O}}^{\text {la }}\right)$ be the Čech complex for $\widetilde{\mathcal{O}}$ and $\widetilde{\mathcal{O}}^{\text {la }}$ with respect to this cover. Then $B \rightarrow C^{\bullet}(\mathfrak{H}, \widetilde{\mathcal{O}})$ is strictly exact by the almost vanishing of higher cohomology (Theorem 1.8.(iv) of [Sch12]). Note that each term in this complex is $\mathfrak{Q} \mathfrak{A}$-acyclic by Theorem 3.6.1. Passing to locally analytic vectors, we see that $B^{\text {la }} \rightarrow C^{\bullet}\left(\mathfrak{U}, \widetilde{\mathcal{O}}^{\text {la }}\right)$ is also exact by the second part of Lemma 2.2.2. Hence, $\breve{H}^{i}\left(U, \widetilde{\mathcal{O}}^{\text {la }}\right)=0, i \geq 1$.

Now by Corollaire 4, p. 176 of [Gro57], the vanishing of higher Čech cohomology for any $U \in \mathfrak{B}$ implies that $H^{i}\left(U, \widetilde{\mathcal{O}}^{\mathrm{la}}\right)=0, i \geq 1$ and $H^{i}\left(X, \widetilde{\mathcal{O}}^{\text {la }}\right)=\check{H}^{i}\left(X, \widetilde{\mathcal{O}}^{\text {la }}\right)$. Hence, we can compute $H^{i}\left(X, \widetilde{\mathcal{O}}^{\text {la }}\right)$ using a Čech complex with respect to a finite cover in $\mathfrak{B}$. We can use $\mathfrak{U}_{0}$ here. All of the claims in the corollary follow on applying the third part of Lemma 2.2.2 to $B \rightarrow C^{\bullet}\left(\mathfrak{U}_{0}, \widetilde{\mathcal{O}}\right)$.

\section{Locally analytic functions on perfectoid modular curves}

Now we apply the previous general theory to the case of modular curves of infinite level at $p$. It turns out that in this case, the differential operator in Theorem 3.1.2 is very classical (see Theorem 4.2.7) and (twisted) $\mathscr{D}$-modules on the flag variety appear naturally in this setup. Following Berger-Colmez [BC16], we also give explicit descriptions of the $\mathrm{GL}_{2}\left(\mathbb{Q}_{p}\right)$-locally analytic functions on the infinite level modular curve. This will be important for our calculations in the next section.

In the first subsection, we will collect some basic facts about modular curves of infinite level at $p$ and the Hodge-Tate period map. This is the simplest case in the theory of perfectoid Shimura varieties developed by Scholze in [Sch15].

\subsection{Modular curves and the Hodge-Tate period map}

4.1.1. We define modular curves adèlically. Fix a neat open compact subgroup $K \subset \mathrm{GL}_{2}\left(\mathbb{A}_{f}\right)$ and let $Y_{K} / \mathbb{Q}$ be the moduli space of elliptic curves with level- $K$ structure. Let $\mathbb{H}^{ \pm 1}$ be the union of upper and lower half-planes. The complex points of $Y_{K}$ are given by the usual double quotient

$$
Y_{K}(\mathbb{C})=\mathrm{GL}_{2}(\mathbb{Q}) \backslash\left(\mathbb{H}^{ \pm 1} \times \mathrm{GL}_{2}\left(\mathbb{A}_{f}\right) / K\right) .
$$

$Y_{K}$ admits a natural compactification $X_{K} / \mathbb{Q}$ by adding finitely many cusps. The universal elliptic curve over $Y_{K}$ extends to a semi-abelian variety over $X_{K}$ and we denote by $\omega$ the sheaf of its invariant differentials. On the complex points, $\omega$ is the canonical extension of $\left.\omega\right|_{Y_{K}(\mathbb{C})}$ as defined in Main Theorem 3.1 of [Mum77].

Fix a complete algebraically closed non-Archimedean field extension $C$ of $\mathbb{Q}_{p}$ as in Section 3.1.1. Denote by $\mathcal{X}_{K}$ (respectively $\mathcal{Y}_{K}$ ) the adic space associated to $X_{K} \times_{\mathbb{Q}} C$ (respectively $Y_{K} \times_{\mathbb{Q}} C$ ).

Theorem 4.1.2. For any tame level $K^{p} \subset \mathrm{GL}_{2}\left(\mathbb{A}_{f}^{p}\right)$ contained in the level-N subgroup $\left\{g \in \mathrm{GL}_{2}\left(\hat{\mathbb{Z}}^{p}\right)=\right.$ $\left.\prod_{l \neq p} \mathrm{GL}_{2}\left(\mathbb{Z}_{l}\right) \mid g \equiv 1 \bmod N\right\}$ for some $N \geq 3$ prime to $p$, there exists a unique perfectoid space $\mathcal{X}_{K}{ }^{p}$ over $C$ such that

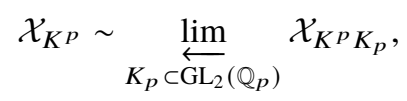


where $K_{p}$ runs through all open compact subgroups of $\mathrm{GL}_{2}\left(\mathbb{Q}_{p}\right)$. Therefore, there is a natural right action of $\mathrm{GL}_{2}\left(\mathbb{Q}_{p}\right)$ on $\mathcal{X}_{K^{p}}$. Moreover, for any open compact subgroup $K_{p}$ of $\mathrm{GL}_{2}\left(\mathbb{Q}_{p}\right)$, there is a basis consisting of open affinoid subsets $U$ of $\mathcal{X}_{K^{p} K_{p}}$ with affinoid perfectoid preimage $U_{\infty}$ in $\mathcal{X}_{K^{p}}$, and the map

$$
\underset{K_{p}^{\prime} \subset K_{p}}{\stackrel{\lim }{\longrightarrow}} H^{0}\left(U_{K_{p}^{\prime}}, \mathcal{O}_{\mathcal{X}_{K^{p} K_{p}^{\prime}}}\right) \rightarrow H^{0}\left(U_{\infty}, \mathcal{O}_{\mathcal{X}_{K} p}\right)
$$

has dense image. Here $U_{K_{p}^{\prime}}$ denotes the preimage of $U$ in $\mathcal{X}_{K^{p}} K_{p}^{\prime}$.

Proof. The existence of $\mathcal{X}_{K^{p}}$ basically follows from Theorem III.1.2 of [Sch15] by taking connected components into account. For the existence of a basis of open subsets of $\mathcal{X}_{K^{p}} K_{p}$, by part (iii) of Theorem III.1.2 of [Sch15], for sufficiently small $K_{p}$, we can find an open cover of $\mathcal{X}_{K^{p} K_{p}}$ with the desired density property. As taking rational subsets preserves this density property by Lemma 4.5 of [Sch13a], we may find such a basis $\mathfrak{B}_{K_{p}}$ of open affinoid subsets for sufficiently small $K_{p}$. In general, we can descend this property to any $K_{p}$. To see this, it is enough to find an affinoid cover of $\mathcal{X}_{K^{p}} K_{p}$ with the desired properties. We may assume $K_{p}$ is a subgroup of $\mathrm{GL}_{2}\left(\mathbb{Z}_{p}\right)$. Take an $\mathrm{GL}_{2}\left(\mathbb{Z}_{p}\right)$-invariant affinoid cover of $\mathscr{F} \ell$. Then Scholze's result implies that this cover comes from an affinoid cover of $\mathcal{X}_{K^{p} K_{p}^{\prime}}$ for some $K_{p}^{\prime}$ sufficiently small. We may assume $K_{p}^{\prime}$ is a normal subgroup of $K_{p}$. Hence, $\mathcal{X}_{K^{p}} K_{p}$ can be viewed as the quotient of $\mathcal{X}_{K^{p} K_{p}^{\prime}}$ by $K_{p} / K_{p}^{\prime}$ and we can descend this cover to $\mathcal{X}_{K^{p} K_{p}}$ by [Han16, Theorem 1.3].

4.1.3. One powerful tool to study the geometry of $\mathcal{X}_{K^{p}}$ is the Hodge-Tate period map introduced in Theorem III.1.2 of [Sch15]. We will give an equivalent definition in our setup.

Fix a $K^{p}$ as in the theorem and an open subgroup $K_{p}$ of $\mathrm{GL}_{2}\left(\mathbb{Z}_{p}\right)$. Let $\mathcal{X}=\mathcal{X}_{K}, \mathcal{Y}=\mathcal{Y}_{K}$ with $K=K^{p} K_{p}$ and let $f: \mathcal{E} \rightarrow \mathcal{Y}$ be the universal elliptic curve. Then $R^{1} f_{*} \mathbb{Z}_{p}$ defines a rank 2 étale $\mathbb{Z}_{p}$-local system $\underline{V}$. Our normalisation is that

○ $\underline{V}$ corresponds to the standard representation of $K_{p} \subset \mathrm{GL}_{2}\left(\mathbb{Z}_{p}\right)$. See, for example, Subsection 2.4 of [Eme06a].

This $\mathbb{Z}_{p}$-local system $\underline{V}$ induces a $\hat{\mathbb{Z}}_{p}$-local system $\underline{\hat{V}}$ on $\mathcal{Y}_{\text {proét }}$, the pro-étale site of $\mathcal{Y}$; see $8.1,8.2$ of [Sch13a] for the notation here. On the other hand, we denote by $D_{\mathrm{dR}}$ the relative de Rham cohomology of $\mathcal{E}$ over $\mathcal{Y}$. This is a rank 2 vector bundle on $\mathcal{Y}$ equipped with a (decreasing) Hodge filtration Fil ${ }^{\bullet}$ and Gauss-Manin connection $\nabla_{\underline{V}}$. Its graded components are given by

$$
\operatorname{gr}^{n}\left(D_{\mathrm{dR}}\right) \cong\left\{\begin{array}{l}
\omega^{-1} \mid \mathcal{Y}, n=0 \\
\omega \mid \mathcal{Y}, n=1 \\
0, n \neq 0,1
\end{array} .\right.
$$

Here implicitly we choose a polarisation of $\mathcal{E}$ - that is, an isomorphism $\wedge^{2} D_{\mathrm{dR}} \cong \mathcal{O}_{\mathcal{Y}}$ - and we will fix this choice from now on. Recall that there is the structural de Rham sheaf $\mathcal{O} \mathbb{B}_{\mathrm{dR}}$ on $\mathcal{Y}_{\text {proét }}$ also equipped with a decreasing filtration and a $\mathbb{B}_{\mathrm{dR}}$-linear connection; cf. $\S 6$ of [Sch13a] and [Sch16]. By the relative de Rham comparison theorem (Theorem 8.8 of [Sch13a]), there is a natural isomorphism

$$
\underline{\hat{V}} \otimes_{\hat{\mathbb{Z}}_{p}} \mathcal{O} \mathbb{B}_{\mathrm{dR}} \cong D_{\mathrm{dR}} \otimes_{\mathcal{O}_{\mathcal{Y}}} \mathcal{O} \mathbb{B}_{\mathrm{dR}}
$$

compatible with the filtrations and connections. Here, by abuse of notation, $D_{\mathrm{dR}}$ is viewed as a sheaf on

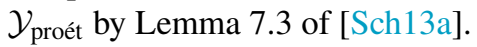

Next, we extend these results to $\mathcal{X}$ using the theory of log adic spaces. See [DLLZ19, DLLZ18] for more details here. We will view $\mathcal{X}$ as a $\log$ adic space by equipping it with the natural $\log$ structure defined by the cusps $\mathcal{C}:=\mathcal{X}-\mathcal{Y}$; cf. Example 2.1.2 of [DLLZ18]. Then $\underline{\hat{V}}$ defines a rank $2 \hat{\mathbb{Z}}_{p}$-local system $\underline{\hat{V}}_{\log }$ on $\mathcal{X}_{\text {prokét }}$, the pro-Kummer étale site of $\mathcal{X}$. There is also the structural de Rham sheaf $\mathcal{O} \mathbb{B}_{\mathrm{dR}, \log }$ on $\mathcal{X}_{\text {prokét }}$ equipped with a decreasing filtration and a logarithmic connection:

$$
\nabla: \mathcal{O} \mathbb{B}_{\mathrm{dR}, \log } \rightarrow \mathcal{O} \mathbb{B}_{\mathrm{dR}, \log } \otimes_{\mathcal{O}_{\mathcal{X}}} \Omega_{\mathcal{X}}^{1}(\mathcal{C})
$$


Here $\Omega_{\mathcal{X}}^{1}(\mathcal{C})$ as usual denotes the sheaf of differentials on $\mathcal{X}$ with simple poles at $\mathcal{C}$. As the monodromy of $\underline{V}$ along each cusp is unipotent, we have the following relative log de Rham comparison isomorphism (Theorem 3.2.12 and its proof of [DLLZ18]):

$$
\hat{\underline{V}}_{\mathrm{log}} \otimes_{\hat{\mathbb{Z}}_{p}} \mathcal{O} \mathbb{B}_{\mathrm{dR}, \log } \cong D_{\mathrm{dR}, \log } \otimes_{\mathcal{O}_{\mathcal{X}}} \mathcal{O} \mathbb{B}_{\mathrm{dR}, \log }
$$

compatible with the filtrations and logarithmic connections, where $D_{\mathrm{dR}, \log }$ is a filtered vector bundle equipped with a logarithmic connection $\nabla_{\underline{V}}$. In fact, by Theorem 1.7 of [DLLZ18], $\left(D_{\mathrm{dR}, \log }, \nabla_{\underline{V}}\right)$ is the canonical extension of $\left(D_{\mathrm{dR}}, \nabla_{\underline{V}}\right)$. Hence, in particular,

$$
\operatorname{gr}^{n}\left(D_{\mathrm{dR}, \log }\right) \cong\left\{\begin{array}{l}
\omega^{-1}, n=0 \\
\omega, n=1 \\
0, n \neq 0,1
\end{array}\right.
$$

Now we have a decreasing filtration (the relative Hodge-Tate filtration) on $\hat{V}_{\log } \otimes_{\hat{\mathbb{Z}}_{p}} \hat{\mathcal{O}}_{\mathcal{X}}$ as in Subsection 2.2. of [CS17]. More precisely, let $\mathcal{O B}_{\mathrm{dR} \text {,log }}^{+}$be the positive structural de Rham sheaf on $\mathcal{X}_{\text {prokét }}$ (the geometric de Rham period sheaf in Definition 2.2.10 of [DLLZ18]) which also admits a logarithmic connection. Consider

$$
\mathbb{M}_{0}=\left(D_{\mathrm{dR}, \log } \otimes_{\mathcal{O}_{\mathcal{X}}} \mathcal{O} \mathbb{B}_{\mathrm{dR}, \log }^{+}\right)^{\nabla=0} .
$$

Recall that there is the positive de Rham sheaf $\mathbb{B}_{\mathrm{dR}}^{+}$on $\mathcal{X}_{\text {prokét }}$ (Definition 2.2.3.(2) of [DLLZ18]) and the usual surjective map: $\theta: \mathbb{B}_{\mathrm{dR}}^{+} \rightarrow \hat{\mathcal{O}}_{\mathcal{X}}$. Then Proposition 7.9 of [Sch13a] implies

$$
\underline{\hat{V}}_{\log } \otimes_{\hat{\mathbb{Z}}_{p}} \mathbb{B}_{\mathrm{dR}, \log }^{+} \supseteq \mathbb{M}_{0} \supseteq \underline{\hat{V}}_{\log } \otimes_{\hat{\mathbb{Z}}_{p}} \operatorname{ker}(\theta) .
$$

Hence, we obtain a filtration on $\underline{\hat{V}}_{\log } \otimes_{\hat{\mathbb{Z}}_{p}} \mathbb{B}_{\mathrm{dR}}^{+} /(\operatorname{ker} \theta)=\underline{\hat{V}}_{\log } \otimes_{\hat{\mathbb{Z}}_{p}} \hat{\mathcal{O}}_{\mathcal{X}}$, which again by Proposition 7.9 of [Sch13a] can be identified with

$$
0 \rightarrow \operatorname{gr}^{0}\left(D_{\mathrm{dR}, \log }\right) \otimes_{\mathcal{O}_{\mathcal{X}}} \hat{\mathcal{O}}_{\mathcal{X}} \rightarrow \underline{\hat{V}}_{\log } \otimes_{\hat{\mathbb{Z}}_{p}} \hat{\mathcal{O}}_{\mathcal{X}} \rightarrow \operatorname{gr}^{1}\left(D_{\mathrm{dR}, \log }\right) \otimes_{\mathcal{O}_{\mathcal{X}}} \hat{\mathcal{O}}_{\mathcal{X}}(-1) \rightarrow 0
$$

In other words, we get the following exact sequence of locally free $\hat{\mathcal{O}}_{\mathcal{X}}$-modules on $\mathcal{X}_{\text {prokét }}$ (relative Hodge-Tate filtration of $\underline{\hat{V}}_{\log } \otimes_{\hat{\mathbb{Z}}_{p}} \hat{\mathcal{O}}_{\mathcal{X}}$ ):

$$
0 \rightarrow \omega^{-1} \otimes_{\mathcal{O}_{\mathcal{X}}} \hat{\mathcal{O}}_{\mathcal{X}}(1) \rightarrow \underline{\hat{V}}_{\log }(1) \otimes_{\hat{\mathbb{Z}}_{p}} \hat{\mathcal{O}}_{\mathcal{X}} \rightarrow \omega \otimes_{\mathcal{O}_{\mathcal{X}}} \hat{\mathcal{O}}_{\mathcal{X}} \rightarrow 0
$$

Remark 4.1.4. Strictly speaking, Proposition 7.9 of [Sch13a] is only proved when there is no $\log$ structure. However, all of the arguments work here with the input replaced by the corresponding results in Section 2 of [DLLZ18]. For example, the local structure of $\mathcal{O B}_{\mathrm{dR}, \log }^{+}$in Proposition 6.10 is now replaced by Proposition 2.3.15 of [DLLZ18].

There is one minor subtlety here: compared with the definition of $\mathcal{O} \mathbb{B}_{\mathrm{dR}}$ in [Sch13a], there is an extra completion of $\mathcal{O B}_{\mathrm{dR}, \log }^{+}\left[t^{-1}\right]$ in Definition 2.2.10 of [DLLZ18], where $t \in \mathbb{B}_{\mathrm{dR}}^{+}$is a generator of $\operatorname{ker}(\theta)$. I claim that such a completion is unnecessary in our case; that is, the isomorphism (4.1.1) can be restricted to

$$
\hat{\underline{V}}_{\log } \otimes_{\hat{\mathbb{Z}}_{p}} \mathcal{O} \mathbb{B}_{\mathrm{dR}, \log }^{+}\left[t^{-1}\right] \cong D_{\mathrm{dR}, \log } \otimes_{\mathcal{O}_{\mathcal{X}}} \mathcal{O} \mathbb{B}_{\mathrm{dR}, \log }^{+}\left[t^{-1}\right] .
$$

In fact, using the local structure of $\mathcal{O B}_{\mathrm{dR}, \log }^{+}$, we may argue as in Theorem 7.2 of [Sch13a] that $\mathbb{M}_{0}$ is a $\mathbb{B}_{\mathrm{dR}}^{+}$-lattice of $\left(\underline{\hat{V}}_{\mathrm{log}} \otimes_{\hat{\mathbb{Z}}_{p}} \mathcal{O} \mathbb{B}_{\mathrm{dR}, \log }\right)^{\nabla=0}=\underline{\hat{V}}_{\mathrm{log}} \otimes_{\hat{\mathbb{Z}}_{p}} \mathbb{B}_{\mathrm{dR}}$ by the Poincaré lemma (Corollary 2.4.2 of [DLLZ18]). This is enough to deduce our claim.

4.1.5. Now we take $K_{p}=\mathrm{GL}_{2}\left(\mathbb{Z}_{p}\right)$. Hence, $\mathcal{X}=\mathcal{X}_{K^{p} \mathrm{GL}_{2}\left(\mathbb{Z}_{p}\right)}$. As $\lim _{K_{p} \subset \mathrm{GL}_{2}\left(\mathbb{Q}_{p}\right)} \mathcal{X}_{K^{p} K_{p}}$ (equipped with $\log$ structures defined by cusps) can be viewed as an open covering of $\mathcal{X}$ in $\mathcal{X}_{\text {prokét }}$, we can evaluate 
the exact sequence (4.1.3) on $\lim _{K_{p}} \mathcal{X}_{K^{p} K_{p}}$. Note that $\mathcal{X}_{K^{p}}$ trivialises the universal Tate module; hence, by Theorem 4.1.2, we obtain the following exact sequence of vector bundles on $\mathcal{X}_{K^{p}}$.

\section{Theorem 4.1.6.}

$$
0 \rightarrow \omega_{K^{p}}^{-1}(1) \rightarrow V(1) \otimes_{\mathbb{Q}_{p}} \mathcal{O}_{\mathcal{X}_{K} p} \rightarrow \omega_{K} p \rightarrow 0,
$$

where $\omega_{K^{p}}$ is the pullback of $\omega$ as a coherent sheaf from $\mathcal{X}$ to $\mathcal{X}_{K^{p}}$ and $V=\mathbb{Q}_{p}^{\oplus 2}$ is the standard representation of $\mathrm{GL}_{2}\left(\mathbb{Q}_{p}\right)$ and viewed as a constant sheaf on $\mathcal{X}_{K^{p}}$. This exact sequence is $\mathrm{GL}_{2}\left(\mathbb{Z}_{p}\right)$ equivariant.

Clearly, the position of $\omega_{K^{p}}^{-1}$ in $V \otimes_{\mathbb{Q}_{p}} \mathcal{O}_{\mathcal{X}_{K} p}$ induces a map (Hodge-Tate period map)

$$
\pi_{\mathrm{HT}}: \mathcal{X}_{K^{p}} \rightarrow \mathscr{F} \ell,
$$

where $\mathscr{F} \ell$ is the adic space over $C$ associated to the usual flag variety for $\mathrm{GL}_{2}$. One can check that our definition of $\pi_{\mathrm{HT}}$ agrees with the one defined in III.3 of [Sch15], ${ }^{1}$ using Lemma III.3.4 and Corollary III.3.17 in the reference.

There is a right action of $\mathrm{GL}_{2}\left(\mathbb{Q}_{p}\right)$ on $\mathscr{F} \ell$ by acting on the total space (rather than the position of the flag). We will always use this right action.

Theorem III.3.18 of [Sch15] provides an affinoid cover $\left\{U_{1}, U_{2}\right\}$ of $\mathscr{F} \ell$. In our case, $\mathscr{F} \ell=\mathbb{P}^{1}$ and $U_{1}=\left\{\left[x_{1}: x_{2}\right],\left\|x_{1}\right\| \geq\left\|x_{2}\right\|\right\}, U_{2}=\left\{\left[x_{1}: x_{2}\right],\left\|x_{2}\right\| \geq\left\|x_{1}\right\|\right\}$.

Theorem 4.1.7. $\pi_{\mathrm{HT}}$ is $\mathrm{GL}_{2}\left(\mathbb{Q}_{p}\right)$ )-equivariant and commutes with Hecke operators away from $p$ (when changing $K^{p}$ ), for the trivial action of these Hecke operators on $\mathscr{F} \ell$. Moreover, let $\mathfrak{B}$ be the set of finite intersections of rational subsets of $U_{1}, U_{2}$. Then $\mathfrak{B}$ is a basis of open affinoid subsets of $\mathscr{F} \ell$ stable under finite intersections and each $U \in \mathfrak{B}$ has the following properties:

○ its preimage $V_{\infty}=\pi_{\mathrm{HT}}^{-1}(U)$ is affinoid perfectoid;

$\circ V_{\infty}$ is the preimage of an affinoid subset $V_{K_{p}} \subset \mathcal{X}_{K^{p} K_{p}}$ for sufficiently small open subgroup $K_{p}$ of $\mathrm{GL}_{2}\left(\mathbb{Q}_{p}\right)$;

○ the map $\lim _{\longrightarrow} H^{0}\left(V_{K_{p}}, \mathcal{O}_{\mathcal{X}_{K}{ }_{K_{p}}}\right) \rightarrow H^{0}\left(V_{\infty}, \mathcal{O}_{\mathcal{X}_{K} p}\right)$ has dense image;

○ $U$ does not contain all $\mathbb{Q}_{p}$-rational points of $\mathscr{F} \ell$.

Proof. See Theorem III.1.2 of [Sch15] and Theorem III.3.18 of [Sch15].

\subsection{Faltings's extension and computation of $\theta$}

Let $K_{p} \subset \mathrm{GL}_{2}\left(\mathbb{Q}_{p}\right)$ be an open subgroup and $X$ an affinoid subset of $\mathcal{X}=\mathcal{X}_{K^{p} K_{p}}$ containing at most one cusp. Suppose its preimage $\tilde{X}$ in $\mathcal{X}_{K^{p}}$ is a ' $\log K_{p}$-Galois pro-étale perfectoid covering' of $X$ and $X$ is small in the sense of Section 3.1.1. Note that by Theorem 4.1.2 and Lemma 5.2 (and its proof) of [Sch15], such $X$ form a basis of open subsets of $\mathcal{X}$.

The goal of this subsection is to compute the differential operator $\theta$ in Theorem 3.1.2 for this Galois covering. It turns out that this follows from a classical result of Faltings which (up to a twist) identifies the relative Hodge-Tate filtration sequence in Theorem 4.1.6 with (log) Faltings's extension; cf. Theorem 5 of [Fal87]. We will give a proof of this result (Theorem 4.2.2) in our setup below. A more conceptual computation of $\theta$ in terms of the $p$-adic Simpson correspondence is given in Remark 4.2.5.

4.2.1. We use notation introduced in the previous subsection. First we recall the log Faltings's extension as defined in Corollary 6.14 of [Sch13a] and Corollary 2.4.5 of [DLLZ18]. Taking the first graded piece

\footnotetext{
${ }^{1}$ There is a slight difference: in our setup, we trivialise the first relative étale cohomology the universal elliptic curve, while [Sch15] trivialises the universal Tate module. As a result, there is a Tate twist in the middle term of the exact sequence in Theorem 4.1.6.
} 
of the Poincaré lemma sequence (Corollary 2.4.2 of [DLLZ18]):

$$
0 \rightarrow \mathbb{B}_{\mathrm{dR}}^{+} \rightarrow \mathcal{O} \mathbb{B}_{\mathrm{dR}, \log }^{+} \stackrel{\nabla}{\rightarrow} \mathcal{O} \mathbb{B}_{\mathrm{dR}, \log }^{+} \otimes_{\mathcal{O}_{\mathcal{X}}} \Omega_{\mathcal{X}}^{1}(\mathcal{C}) \rightarrow 0,
$$

we obtain an exact sequence of $\hat{\mathcal{O}}_{\mathcal{X}}$-modules on $\mathcal{X}_{\text {prokét }}$ (the log Faltings's extension):

$$
0 \rightarrow \hat{\mathcal{O}}_{\mathcal{X}}(1) \rightarrow \operatorname{gr}^{1} \mathcal{O B}_{\mathrm{dR}, \log }^{+} \stackrel{\nabla}{\rightarrow} \hat{\mathcal{O}}_{\mathcal{X}} \otimes_{\mathcal{O}_{\mathcal{X}}} \Omega_{\mathcal{X}}^{1}(\mathcal{C}) \rightarrow 0
$$

Its tensor product with $\omega^{-1}$ becomes

$$
0 \rightarrow \omega^{-1} \otimes_{\mathcal{O}_{\mathcal{X}}} \hat{\mathcal{O}}_{\mathcal{X}}(1) \rightarrow \omega^{-1} \otimes_{\mathcal{O}_{\mathcal{X}}} \mathrm{gr}^{1} \mathcal{O B}_{\mathrm{dR}, \log }^{+} \rightarrow \omega^{-1} \otimes_{\mathcal{O}_{\mathcal{X}}} \Omega_{\mathcal{X}}^{1}(\mathcal{C}) \otimes_{\mathcal{O}_{\mathcal{X}}} \hat{\mathcal{O}}_{\mathcal{X}} \rightarrow 0
$$

Recall that there is a Kodaira-Spencer map $\omega \rightarrow \omega^{-1} \otimes_{\mathcal{O}_{\mathcal{X}}} \Omega_{\mathcal{X}}^{1}(\mathcal{C})$ of coherent sheaves on $\mathcal{X}$ defined as the composite

$$
\omega=\mathrm{Fil}^{1} D_{\mathrm{dR}, \log } \subset D_{\mathrm{dR}, \log } \stackrel{\nabla}{\rightarrow} D_{\mathrm{dR}, \log } \otimes_{\mathcal{O}_{\mathcal{X}}} \Omega_{\mathcal{X}}^{1}(\mathcal{C}) \rightarrow \operatorname{gr}^{0} D_{\mathrm{dR}, \log } \otimes_{\mathcal{O}_{\mathcal{X}}} \Omega_{\mathcal{X}}^{1}(\mathcal{C})=\omega^{-1} \otimes_{\mathcal{O}_{\mathcal{X}}} \Omega_{\mathcal{X}}^{1}(\mathcal{C}) .
$$

It is well-known that this is an isomorphism: $\omega \stackrel{\sim}{\rightarrow} \omega^{-1} \otimes_{\mathcal{O}_{\mathcal{X}}} \Omega_{\mathcal{X}}^{1}(\mathcal{C})$. Under this isomorphism, $\omega^{-1} \otimes_{\mathcal{O}_{\mathcal{X}}}$ $\mathrm{gr}^{1} \mathcal{O} \mathbb{B}_{\mathrm{dR}, \log }^{+}$can be viewed as an element of

$$
\operatorname{Ext}_{\mathcal{X}_{\text {prokét }}}^{1}\left(\omega \otimes_{\mathcal{O}_{\mathcal{X}}} \hat{\mathcal{O}}_{\mathcal{X}}, \omega^{-1} \otimes_{\mathcal{O}_{\mathcal{X}}} \hat{\mathcal{O}}_{\mathcal{X}}(1)\right)
$$

On the other hand, recall that $\hat{V}_{\log } \otimes_{\hat{\mathbb{Z}}_{p}} \hat{\mathcal{O}}_{\mathcal{X}}$ also defines an element in this extension group by the relative Hodge-Tate filtration (4.1.3).

Theorem 4.2.2. There is an isomorphism between $\omega^{-1} \otimes_{\mathcal{O}_{\mathcal{X}}} \operatorname{gr}^{1} \mathcal{O B}_{\mathrm{dR}, \log }^{+}$and $\underline{\hat{V}}_{\log }(1) \otimes_{\hat{\mathbb{Z}}_{p}} \hat{\mathcal{O}}_{\mathcal{X}}$ as $\hat{\mathcal{O}}_{\mathcal{X}^{-}}$ modules such that as extension classes, they differ by -1 .

Proof. Consider the first graded piece of the tensor product of $\underline{\underline{V}}_{\log }$ and the Poincaré lemma sequence (4.2.1) over $\hat{\mathbb{Z}}_{p}$; that is, the tensor product of $\underline{\hat{V}}_{\log }$ with the log Faltings's extension:

$$
0 \rightarrow \underline{\hat{V}}_{\log }(1) \otimes_{\hat{\mathbb{Z}}_{p}} \hat{\mathcal{O}}_{\mathcal{X}} \rightarrow \underline{\hat{V}}_{\log } \otimes_{\hat{\mathbb{Z}}_{p}} \operatorname{gr}^{1} \mathcal{O} \mathbb{B}_{\mathrm{dR}, \log }^{+} \stackrel{\nabla}{\rightarrow} \underline{\hat{V}}_{\log } \otimes_{\hat{\mathbb{Z}}_{p}} \hat{\mathcal{O}}_{\mathcal{X}} \otimes_{\mathcal{O}_{\mathcal{X}}} \Omega_{\mathcal{X}}^{1}(\mathcal{C}) \rightarrow 0 .
$$

Then the inclusion $\operatorname{gr}^{1}\left(D_{\mathrm{dR}, \log } \otimes_{\mathcal{O}_{\mathcal{X}}} \mathcal{O} \mathbb{B}_{\mathrm{dR}, \log }^{+}\right) \subset \operatorname{gr}^{1}\left(\underline{\hat{V}}_{\log } \otimes_{\hat{\mathbb{Z}}_{p}} \mathcal{O} \mathbb{B}_{\mathrm{dR}, \log }^{+}\right)$induces

$$
0 \rightarrow \hat{V}_{\mathrm{log}}(1) \otimes_{\hat{\mathbb{Z}}_{p}} \hat{\mathcal{O}}_{\mathcal{X}} \rightarrow \operatorname{gr}^{1}\left(D_{\mathrm{dR}, \log } \otimes_{\mathcal{O}_{\mathcal{X}}} \mathcal{O B}_{\mathrm{dR}, \log }^{+}\right) \stackrel{\nabla}{\rightarrow} \operatorname{gr}^{0} D_{\mathrm{dR}, \log } \otimes_{\mathcal{O}_{\mathcal{X}}} \hat{\mathcal{O}}_{\mathcal{X}} \otimes_{\mathcal{O}_{\mathcal{X}}} \Omega_{\mathcal{X}}^{1}(\mathcal{C}),
$$

where the first inclusion follows from the sequence (4.1.2) and the image of $\nabla$ lies in $\mathrm{gr}^{0} D_{\mathrm{dR}, \log } \otimes_{\mathcal{O}_{\mathcal{X}}}$ $\hat{\mathcal{O}}_{\mathcal{X}} \otimes_{\mathcal{O}_{\mathcal{X}}} \Omega_{\mathcal{X}}^{1}(\mathcal{C})$ by Griffiths transversality. I claim that $\nabla$ is in fact surjective as it has a left inverse: the composite map

$$
\operatorname{gr}^{1}\left(D_{\mathrm{dR}, \log }\right) \otimes_{\mathcal{O}_{\mathcal{X}}} \hat{\mathcal{O}}_{\mathcal{X}} \subset \operatorname{gr}^{1}\left(D_{\mathrm{dR}, \log } \otimes_{\mathcal{O}_{\mathcal{X}}} \mathcal{O B}_{\mathrm{dR}, \log }^{+}\right) \stackrel{\nabla}{\rightarrow} \operatorname{gr}^{0} D_{\mathrm{dR}, \log } \otimes_{\mathcal{O}_{\mathcal{X}}} \hat{\mathcal{O}}_{\mathcal{X}} \otimes_{\mathcal{O}_{\mathcal{X}}} \Omega_{\mathcal{X}}^{1}(\mathcal{C})
$$

is nothing but the tensor product of Kodaira-Spencer map with $\hat{\mathcal{O}}_{\mathcal{X}}$ and, hence, an isomorphism. This implies that

$$
\hat{V}_{\log }(1) \otimes_{\hat{\mathbb{Z}}_{p}} \hat{\mathcal{O}}_{\mathcal{X}} \stackrel{\sim}{\rightarrow} \operatorname{gr}^{1}\left(D_{\mathrm{dR}, \log } \otimes_{\mathcal{O}_{\mathcal{X}}} \mathcal{O B}_{\mathrm{dR}, \log }^{+}\right) / \operatorname{gr}^{1}\left(D_{\mathrm{dR}, \log }\right) \otimes_{\mathcal{O}_{\mathcal{X}}} \hat{\mathcal{O}}_{\mathcal{X}}
$$

Note that this right-hand side can be identified with $\operatorname{gr}^{0}\left(D_{\mathrm{dR}, \log }\right) \otimes_{\mathcal{O}_{\mathcal{X}}} \operatorname{gr}^{1} \mathcal{O B}_{\mathrm{dR}, \log }^{+}=\omega^{-1} \otimes_{\mathcal{O}_{\mathcal{X}}}$ $\mathrm{gr}^{1} \mathcal{O} \mathbb{B}_{\mathrm{dR}, \log }^{+}$as there is a canonical decomposition

$$
\operatorname{gr}^{1}\left(D_{\mathrm{dR}, \log } \otimes_{\mathcal{O}_{\mathcal{X}}} \mathcal{O} \mathbb{B}_{\mathrm{dR}, \log }^{+}\right)=\operatorname{gr}^{1}\left(D_{\mathrm{dR}, \log }\right) \otimes_{\mathcal{O}_{\mathcal{X}}} \hat{\mathcal{O}}_{\mathcal{X}} \oplus \operatorname{gr}^{0}\left(D_{\mathrm{dR}, \log }\right) \otimes_{\mathcal{O}_{\mathcal{X}}} \operatorname{gr}^{1} \mathcal{O} \mathbb{B}_{\mathrm{dR}, \log }^{+}
$$


Hence, we obtain an isomorphism $\underline{\hat{V}}_{\mathrm{log}}(1) \otimes_{\hat{\mathbb{Z}}_{p}} \hat{\mathcal{O}}_{\mathcal{X}} \cong \omega^{-1} \otimes_{\mathcal{O}_{\mathcal{X}}} \mathrm{gr}^{1} \mathcal{O} \mathbb{B}_{\mathrm{dR}, \log }^{+}$as claimed in the theorem. To check their relation as extension classes, one useful observation is that the surjection $\underline{\hat{V}}_{\log }(1) \otimes_{\hat{\mathbb{Z}}_{p}} \hat{\mathcal{O}}_{\mathcal{X}} \rightarrow$ $\omega^{1} \otimes_{\mathcal{O}_{\mathcal{X}}} \hat{\mathcal{O}}_{\mathcal{X}}$ in the exact sequence (4.1.3) agrees with the composite map

$$
\underline{\hat{V}}_{\mathrm{log}}(1) \otimes_{\hat{\mathbb{Z}}_{p}} \hat{\mathcal{O}}_{\mathcal{X}} \subset \operatorname{gr}^{1}\left(D_{\mathrm{dR}, \log } \otimes_{\mathcal{O}_{\mathcal{X}}} \mathcal{O} \mathbb{B}_{\mathrm{dR}, \log }^{+}\right) \rightarrow \operatorname{gr}^{1}\left(D_{\mathrm{dR}, \log }\right) \otimes_{\mathcal{O}_{\mathcal{X}}} \hat{\mathcal{O}}_{\mathcal{X}}
$$

where the second map is the projection using the above decomposition. Hence, it differs by -1 with the composite

$$
\begin{aligned}
& \hat{V}_{\log }(1) \otimes_{\hat{\mathbb{Z}}_{p}} \hat{\mathcal{O}}_{\mathcal{X}} \rightarrow \operatorname{gr}^{1}\left(D_{\mathrm{dR}, \log } \otimes_{\mathcal{O}_{\mathcal{X}}} \mathcal{O B}_{\mathrm{dR}, \log }^{+}\right) \rightarrow \operatorname{gr}^{0}\left(D_{\mathrm{dR}, \log }\right) \otimes_{\mathcal{O}_{\mathcal{X}}} \operatorname{gr}^{1} \mathcal{O B}_{\mathrm{dR}, \log }^{+} \\
& \stackrel{\nabla}{\rightarrow} \operatorname{gr}^{0} D_{\mathrm{dR}, \log } \otimes_{\mathcal{O}_{\mathcal{X}}} \hat{\mathcal{O}}_{\mathcal{X}} \otimes_{\mathcal{O}_{\mathcal{X}}} \Omega_{\mathcal{X}}^{1}(\mathcal{C}) \stackrel{\sim}{\leftarrow} \operatorname{gr}^{1}\left(D_{\mathrm{dR}, \log }\right) \otimes_{\mathcal{O}_{\mathcal{X}}} \hat{\mathcal{O}}_{\mathcal{X}},
\end{aligned}
$$

where the second map is the other projection and the last isomorphism is given by Kodaira-Spencer map. Note that the composite of the first row is the isomorphism constructed before. This finishes the proof of theorem.

Corollary 4.2.3. Let $X, \tilde{X}$ be as in the beginning of this subsection. Moreover, we assume $\pi_{\mathrm{HT}}(\tilde{X})$ is contained in an affinoid open subset of $\mathscr{F} \ell$. Then $\tilde{X}$ is a locally analytic covering of $X$ in the sense of Definition 3.5.4.

Proof. By Proposition 3.5.3, one equivalent definition of locally analytic covering is that if we take the global sections of Faltings's extension on $\tilde{X}$ (viewed as open set in $\mathcal{X}_{\text {prokét }}$ by abuse of notation), it remains exact after taking $K_{p}$-locally analytic vectors. First consider taking the global sections of relative Hodge-Tate filtration (4.1.3) on $\tilde{X}$ :

$$
0 \rightarrow H^{0}\left(\tilde{X}, \omega^{-1} \otimes \hat{\mathcal{O}}_{\mathcal{X}}(1)\right) \rightarrow H^{0}\left(\tilde{X}, \underline{V}_{\log }(1) \otimes \hat{\mathcal{O}}_{\mathcal{X}}\right) \rightarrow H^{0}\left(\tilde{X}, \omega \otimes \hat{\mathcal{O}}_{\mathcal{X}}\right) \rightarrow 0 .
$$

This is exact as $H^{1}\left(\tilde{X}, \omega^{-1} \otimes_{\mathcal{O}_{\mathcal{X}}} \hat{\mathcal{O}}_{\mathcal{X}}(1)\right)=0$ by Theorem 5.4.3 of [DLLZ19]. Note that $\underline{\hat{V}}_{\text {log }}$ becomes a trivial local system on $\tilde{X}$; hence, the middle term is naturally isomorphic to $V(1) \otimes_{\mathbb{Q}_{p}} H^{0}\left(\tilde{X}, \hat{\mathcal{O}}_{\mathcal{X}}\right)$ where $V=\mathbb{Q}_{p}^{\oplus 2}$ is the standard representation of $K_{p} \subset \mathrm{GL}_{2}\left(\mathbb{Q}_{p}\right)$. By our assumption on $\pi_{\mathrm{HT}}(\tilde{X})$, there exists an element $\mathbf{e} \in V(1) \otimes_{\mathbb{Q}_{p}} C \subset V(1) \otimes_{\mathbb{Q}_{p}} H^{0}\left(\tilde{X}, \hat{\mathcal{O}}_{\mathcal{X}}\right)$ whose image $e \in H^{0}\left(\tilde{X}, \omega \otimes_{\mathcal{O}_{\mathcal{X}}} \hat{\mathcal{O}}_{\mathcal{X}}\right)$ is a basis of $H^{0}\left(\tilde{X}, \omega \otimes_{\mathcal{O}_{\mathcal{X}}} \hat{\mathcal{O}}_{\mathcal{X}}\right)$ as a $H^{0}\left(\tilde{X}, \hat{\mathcal{O}}_{\mathcal{X}}\right)$-module.

As the action of $K_{p}$ on $V$ is analytic (even algebraic), we conclude that the short exact sequence (4.2.2) remains exact after passing to the $K_{p}$-locally analytic vectors. The same holds when taking global sections of the tensor product of the exact sequence (4.1.3) and $\omega$, which is equivalent to multiplying the sequence (4.2.2) by $e$. Now the corollary follows by identifying this exact sequence with Faltings's extension (up to -1 ).

Now we are ready to calculate $\theta$. We will give an explicit description first and rewrite in a coordinatefree way later. We

$\circ$ fix a generator of $\mathbb{Z}_{p}(1)$ from now on; that is, an isomorphism $\mathbb{Z}_{p}(1) \cong \mathbb{Z}_{p}$.

Let $\mathbf{e}_{1}=(1,0), \mathbf{e}_{2}=(0,1)$ be the standard basis of $V=\mathbb{Q}_{p}^{\oplus 2}$ and denote their images by $e_{1}, e_{2} \in$ $H^{0}\left(\mathcal{X}_{K^{p}}, \omega_{K^{p}}\right)$ (fake-Hasse invariants) using the surjective map in the exact sequence in Theorem 4.1.6 and the chosen generator of $\mathbb{Z}_{p}(1)$. We remark that $\operatorname{Aut}\left(C / \mathbb{Q}_{p}\right)$ acts on $e_{1}, e_{2}$ via the cyclotomic character because of the Tate-twist in $V(1)$.

Let $X, \tilde{X}$ be as in the previous corollary. In particular, $\left.\omega_{K^{p}}\right|_{\tilde{X}}$ is trivial and generated by some section $e$. Hence, we may view $\frac{e_{1}}{e}, \frac{e_{2}}{e}$ as elements in $\mathcal{O}_{\mathcal{X}_{K} p}(\tilde{X})$. 
Theorem 4.2.4. The differential operator $\theta$ associated to $\left(K_{p}, \tilde{X}\right)$ in Theorem 3.1.2 is, up to a unit of $\mathcal{O}_{\mathcal{X}_{K} p}(\tilde{X})$,

$$
\frac{1}{e^{2}}\left(\begin{array}{cc}
e_{1} e_{2} & e_{2}^{2} \\
-e_{1}^{2} & -e_{1} e_{2}
\end{array}\right) \in \mathfrak{g l}_{2}\left(\mathcal{O}_{\mathcal{X}_{K} p}(\tilde{X})\right)=\mathcal{O}_{\mathcal{X}_{K} p}(\tilde{X}) \otimes_{\mathbb{Q}_{p}} \operatorname{Lie}\left(K_{p}\right)
$$

Remark 4.2.5. As mentioned in Remark 3.1.7, it is better to view $\theta$ as a log Higgs field. The computation of $\theta$ here is just computing the Higgs bundle associated to $\underline{\hat{V}}_{\log }$ under the $p$-adic Simpson correspondence, which can be deduced from $\left(D_{\mathrm{dR}, \log }, \nabla\right)$, the other side of the de Rham comparison. This was pointed out to me by Michael Harris.

Proof. We will freely use the notation and constructions introduced in Section 3. Hence, $X=$ $\operatorname{Spa}\left(A, A^{+}\right), \tilde{X}=\operatorname{Spa}\left(B, B^{+}\right)$. Fix an étale map $X \rightarrow Y$ as in Section 3.2.1. Let $X_{\infty}=\operatorname{Spa}\left(A_{\infty}, A_{\infty}^{+}\right)$ (respectively $\left.\tilde{X}_{\infty}=\operatorname{Spa}\left(B_{\infty}, B_{\infty}^{+}\right)\right)$be the pullback of the perfectoid covering $Y_{\infty}$ to $X$ (respectively $\left.\tilde{X}\right)$; cf. Section 3.2.2 (respectively 3.2.6).

To compute $\theta$, we follow Remark 3.3.7. Recall that $V$ is the standard 2-dimensional $\mathbb{Q}_{p}$-representation of $K_{p} \subset \mathrm{GL}_{2}\left(\mathbb{Q}_{p}\right)$. We need to compute $\phi_{V}: \operatorname{Lie}(\Gamma) \rightarrow B_{\infty} \otimes_{\mathbb{Q}_{p}} V$ in Proposition 3.2.13, which agrees with the natural action of $\operatorname{Lie}(\Gamma)$ when restricted to $\left(B_{\infty} \otimes_{\mathbb{Q}_{p}} V\right)^{K_{p}, \Gamma-\text { la }}$. By Theorem 4.1.6, ignoring the Tate-twist, we have

$$
0 \rightarrow B \cdot e^{-1} \rightarrow B \otimes_{\mathbb{Q}_{p}} V \rightarrow B \cdot e \rightarrow 0,
$$

where $e^{-1} \in H^{0}\left(\tilde{X}, \omega_{K^{p}}^{-1}\right)$ is dual to $e \in H^{0}\left(\tilde{X}, \omega_{K^{p}}\right)$. Take the tensor product with $B_{\infty}$ over $B$ and then take the $K_{p}$-invariants:

$$
0 \rightarrow\left(B_{\infty} \cdot e^{-1}\right)^{K_{p}} \rightarrow\left(B_{\infty} \otimes V\right)^{K_{p}} \rightarrow\left(B_{\infty} \cdot e\right)^{K_{p}} \rightarrow 0 .
$$

To compute the action of $\operatorname{Lie}(\Gamma)$ on $\left(B_{\infty} \otimes V\right)^{K_{p}, \Gamma-\mathrm{la}}$, by Theorem 4.2.2, this sequence can be identified with the global sections of Faltings's extension on $X_{\infty}$ (viewed as an open set in $\mathcal{X}_{\text {prokét }}$ ), up to -1 . Hence, by results in Section 2 of [DLLZ18] (in particular, Proposition 2.3.15 and proof of Corollary 2.4.2), the sequence (4.2.3) is $A_{\infty}$-linearly and $\Gamma$-equivariant isomorphic to the tensor product over $\mathbb{Q}_{p}$ of $A_{\infty}$ with a nontrivial self extension of trivial representation of $\Gamma$ :

$$
0 \rightarrow \mathbb{Q}_{p} \rightarrow V_{1} \rightarrow \mathbb{Q}_{p} \rightarrow 0
$$

which we essentially write down in Section 3.5.1. Clearly, Lie $(\Gamma)$ acts trivially on $\left(B_{\infty} \cdot e^{-1}\right)^{K_{p}, \Gamma-\text { la } \cong}$ $\left(A_{\infty}\right)^{\Gamma-\text { la }}$, and if we fix a generator $x$ of $\operatorname{Lie}(\Gamma)$, it maps the quotient $\left(B_{\infty} \cdot e\right)^{K_{p}, \Gamma-\text { la }}$ isomorphically to $\left(B_{\infty} \cdot e^{-1}\right)^{K_{p}, \Gamma-\mathrm{la}}$. Therefore, $\phi_{V}(x)$ is zero on $B \cdot e^{-1}$ and $\phi_{V}(x)(B \otimes V)=B \cdot e^{-1}$.

The rest is to write this as an element of $B \otimes_{\mathbb{Q}_{p}} \operatorname{Lie}\left(K_{p}\right)$ up to $B^{\times}$. It is easy to see that $\mathbf{f}=\frac{e_{2}}{e} \mathbf{e}_{1}-\frac{e_{1}}{e} \mathbf{e}_{2} \in$ $B \otimes_{\mathbb{Q}_{p}} V$ generates $B \cdot e^{-1}$. As $\left.\phi_{V}(x)\right|_{B \otimes V}$ factors through $B \cdot e$, we may assume it sends $e$ to $\mathbf{f}$. A direct computation gives the matrix in the theorem.

4.2.6. To rewrite this theorem in a coordinate-free way, it is better to work with sheaves. Consider the pushforward of $\mathcal{O}_{\mathcal{X}_{K} p}$ along $\pi_{\mathrm{HT}}: \mathcal{X}_{K^{p}} \rightarrow \mathscr{F} \ell$

$$
\mathcal{O}_{K^{p}}:=\pi_{\mathrm{HT} *} \mathcal{O}_{\mathcal{X}_{K} p}
$$

We define a subsheaf

$$
\mathcal{O}_{K^{p}}^{\mathrm{la}} \subset \mathcal{O}_{K^{p}}
$$

as follows: for any quasi-compact open set $U$ of $\mathscr{F} \ell, H^{0}\left(U, \mathcal{O}_{K^{p}}^{\text {la }}\right)$ is the subspace of $K_{p}$-locally analytic vectors in $H^{0}\left(U, \mathcal{O}_{K^{p}}\right)$, where $K_{p}$ is an open subgroup of $\mathrm{GL}_{2}\left(\mathbb{Q}_{p}\right)$ stabilising $U$. Note that such $K_{p}$ 
always exists by the compactness of $U$ and $H^{0}\left(U, \mathcal{O}_{K^{p}}^{\text {la }}\right)$ is independent of choice of $K_{p}$. Since taking locally analytic vectors is a left-exact process (see the construction in Section 2) and $\pi_{\mathrm{HT} *} \mathcal{O}_{\mathcal{X}_{K} p}$ is a sheaf, $\mathcal{O}_{K^{p}}^{\text {la }}$ indeed defines a sheaf on $\mathscr{F} \ell$.

Clearly, $\mathcal{O}_{K^{p}}^{\text {la }}$ is $\mathrm{GL}_{2}\left(\mathbb{Q}_{p}\right)$-equivariant and there is a natural action of the Lie algebra of $\mathrm{GL}_{2}\left(\mathbb{Q}_{p}\right)$ on $\mathcal{O}_{K}^{\text {la }}$. Hecke operators away from $p$ act on it naturally as we only use the action at $p$ in the construction.

We claim that the natural map $\mathcal{O}_{\mathscr{F} \ell} \rightarrow \mathcal{O}_{K^{p}}$ has image in $\mathcal{O}_{K^{p}}^{\text {la }}$. It is enough to check this for a basis of affinoid open subsets $U$ of $\mathscr{F} \ell$. Since $\mathscr{F} \ell$ is an adic space associated to a variety over $C$ of finite type, we may consider all affinoid subsets $U$ such that $\mathcal{O}_{\mathscr{F} \ell}(U)$ can be written as a continuous quotient of $C\left\langle T_{1} \cdots, T_{m}\right\rangle$ for some $m$ and the quotient topology on $\mathcal{O}_{\mathscr{F} \ell}(U)$ agrees with its natural topology. Fix such a $U$; then there exists an open subgroup $K_{p} \subset \mathrm{GL}_{2}\left(\mathbb{Q}_{p}\right)$ stabilising $U$ and acting trivially on $\mathcal{O}_{\mathscr{F} \ell}^{+}(U) / p$. From this, we see that the action of $K_{p}$ on $\mathcal{O}_{\mathscr{F} \ell}(U)$ is locally analytic (using arguments in Example 2.1.9, for example).

We follow some constructions on the flag variety in [BB83]. Denote by $\mathfrak{g}$ the tensor product of the Lie algebra of $\mathrm{GL}_{2}\left(\mathbb{Q}_{p}\right)$ with $C$; that is, $\mathfrak{g l}_{2}(C)$. For a $C$-point $x$ of the flag variety $\mathrm{Fl}$ of $\mathrm{GL}_{2} / C$, let $\mathfrak{b}_{x}, \mathfrak{n}_{x} \subset \mathfrak{g}$ be its corresponding Borel subalgebra and nilpotent subalgebra. Let

$$
\begin{aligned}
\mathfrak{g}^{0} & :=\mathcal{O}_{\mathrm{Fl}} \otimes_{C} \mathfrak{g}, \\
\mathfrak{b}^{0} & :=\left\{f \in \mathfrak{g}^{0} \mid f_{x} \in \mathfrak{b}_{x}, \text { for all } x \in \operatorname{Fl}(C)\right\}, \\
\mathfrak{n}^{0} & :=\left\{f \in \mathfrak{g}^{0} \mid f_{x} \in \mathfrak{n}_{x}, \text { for all } x \in \operatorname{Fl}(C)\right\}
\end{aligned}
$$

be $\mathrm{GL}_{2}$-equivariant vector bundles on $\mathrm{Fl}$, where $\mathrm{GL}_{2}$ acts by conjugation on $\mathfrak{g}$. By abuse of notation, we also view these as vector bundles on $\mathscr{F} \ell$, the associated adic space of Fl. Then we have a natural action of $\mathfrak{g}^{0}$ on $\mathcal{O}_{K^{p}}^{\text {la }}$ : for $f \in \mathcal{O}_{\mathscr{F} \ell}, z \in \mathfrak{g}, s \in \mathcal{O}_{K}^{\mathrm{la}}$,

$$
(f \otimes z) \cdot s=f(z \cdot s) .
$$

Theorem 4.2.7. $\mathfrak{n}^{0}$ acts trivially on $\mathcal{O}_{K^{p}}^{\text {la }}$.

Proof. Its enough to check this on open subsets as in Theorem 4.1.7, for which we may invoke Theorem 4.2.4 and our claim follows immediately. In fact, it follows from the proof of Theorem 4.2.4 that the action of $\phi_{V}$ on $V(1) \otimes_{\mathbb{Q}_{p}} \mathcal{O}_{\mathcal{X}_{K} p}$ is trivial on $\omega_{K^{p}}^{-1}(1)$ and induces an isomorphism $\omega_{K}{ }^{p} \stackrel{\sim}{\rightarrow} \omega_{K^{p}}^{-1}(1)$. Since $\pi_{\mathrm{HT}}$ is defined by the position of $\omega_{K^{p}}^{-1}(1)$ in $V(1) \otimes_{\mathbb{Q}_{p}} \mathcal{O}_{\mathcal{X}_{K} p}$, it is tautological that $\mathcal{O}_{\mathcal{X}_{K} p} \theta \subset \mathcal{O}_{\mathcal{X}_{K} p} \otimes_{C} \mathfrak{g}$ contains $\mathfrak{n}^{0}$.

Fix a rational Borel subalgebra $\mathfrak{b}:=\left\{\left(\begin{array}{ll}* & * \\ 0 & *\end{array}\right)\right\}$ and a Cartan subalgebra $\mathfrak{h}:=\left\{\left(\begin{array}{ll}* & 0 \\ 0 & *\end{array}\right)\right\}$ of $\mathfrak{g}$ and let $W$ be the Weyl group. Recall the Harish-Chandra isomorphism $Z(U(\mathfrak{g})) \cong S(\mathfrak{h})^{W}$, where $Z(U(\mathfrak{g}))$ is the centre of the universal enveloping algebra $U(\mathfrak{g})$ of $\mathfrak{g}$ and $S(\mathfrak{h})$ is the symmetric algebra of $\mathfrak{h}$ equipped with the dot action of $W: w \cdot \mu=w(\mu+\rho)-\rho, \mu \in \mathfrak{h}^{*}$. Here $\rho$ is the half sum of positive roots as usual. Let $\mathfrak{g}_{0}:=\mathfrak{s l}_{2}(C) \subset \mathfrak{g}$ and $\mathfrak{h}_{0}=\mathfrak{h} \cap \mathfrak{g}_{0}$. Then, similarly, $Z\left(U\left(\mathfrak{g}_{5}\right)\right) \cong S\left(\mathfrak{h}_{5}\right)^{W}$. We also denote by $\mathfrak{z}:=\left\{\left(\begin{array}{ll}a & 0 \\ 0 & a\end{array}\right)\right\} \subset \mathfrak{g}$ the centre of $\mathfrak{g}$.

It is clear that $\mathfrak{b}^{0} / \mathfrak{n}^{0}=\mathcal{O}_{\mathscr{H} \ell} \otimes_{C} \mathfrak{h}$ by identifying global sections of $\mathfrak{b}^{0} / \mathfrak{n}^{0}$ with $\mathfrak{h}$. So we have a a natural embedding $\mathfrak{h} \rightarrow \mathfrak{b}^{0} / \mathfrak{n}^{0}$.

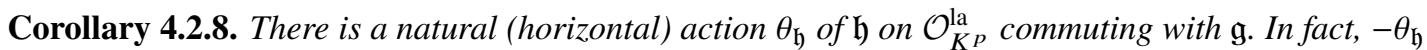
induces an action of $S\left(\mathfrak{h}_{5}\right)$ extending the one of $Z\left(U\left(\mathfrak{g}_{5}\right)\right)$ and $\left.\theta_{\mathfrak{h}}\right|_{\mathfrak{z}}$ agrees with the action of $\mathfrak{z} \subset \mathfrak{g}$.

Proof. The first part follows from the fact that $H^{0}\left(\mathscr{F} \ell, \mathfrak{b}^{0} / \mathfrak{n}^{0}\right)$ is $\mathrm{GL}_{2}$-invariant. The second part essentially follows from Harish-Chandra's theorem. Note that there is a sign here since this construction studies the $\mathfrak{n}_{x}$-coinvariants rather than invariants. See Section 2 and Section 3 of [BB83]. 
Remark 4.2.9. See Theorem 5.1.8, 5.1.11 for an interpretation of this action in terms of the classical Sen operator arising from the action of $G_{\mathbb{Q}_{p}}$.

Remark 4.2.10. In Beilinson-Bernstein's theory of localisation (see, for example, Section C of [Ber84]), $\mathcal{O}_{K^{p}}^{\text {la }}$ is a $\tilde{\mathscr{D}}$-module in this analytic setting. Similarly, fix a weight $\chi$ of $\mathfrak{h}$. We denote by $\mathcal{O}_{K^{p}}^{\mathrm{la}, \chi}$ the $\chi^{-}$ component of $\mathcal{O}_{K^{p}}^{\text {la }}$; that is, the subsheaf of sections of weight $\chi$. Then $\mathcal{O}_{K^{p}}^{\text {la, } \chi}$ is a $\tilde{\mathscr{D}}_{\chi^{-} \text {-module. Recall }}$ that $\tilde{\mathscr{D}}_{\chi}$ is a ring of twisted differential operators on the flag variety. Everything here is also $\mathrm{GL}_{2}\left(\mathbb{Q}_{p}\right)$ equivariant. Hence, we obtain a $\left(\tilde{\mathscr{D}}_{\chi}, \mathrm{GL}_{2}\left(\mathbb{Q}_{p}\right)\right)$-module on $\mathscr{F} \ell$, which is very similar to the picture in the complex analytic setting.

In a series of works (see, for example, [Ard17]), Ardakov proved a $p$-adic analogue of BeilinsonBernstein's localisation theorem. Roughly speaking, he showed that one can realise the dual of an admissible locally analytic representation of $\mathrm{GL}_{2}\left(\mathbb{Q}_{p}\right)$ as global sections of certain $\mathrm{GL}_{2}\left(\mathbb{Q}_{p}\right)$-equivariant $\mathscr{D}$-modules on $\mathscr{F l}$. It is very natural to ask whether there is some form of duality relating his construction with $\mathcal{O}_{K^{p}}^{\text {la, } \chi}$ here.

\subsection{Local structure of $\mathcal{O}_{K^{p}}^{\mathrm{la}}$}

So far, $\mathcal{O}_{K^{p}}^{\text {la }}$ is defined in an abstract way. We give an explicit description of its sections in this subsection. This is largely inspired by the calculations of Berger-Colmez in Section 4 and Section 5 of [BC16]. We will follow their arguments and combine with our differential equation $\theta=0$. To do this, one needs enough sections of $\mathcal{O}_{K^{p}}^{\text {la }}$.

4.3.1. Let $\Gamma\left(p^{n}\right)=1+p^{n} M_{2}\left(\mathbb{Z}_{p}\right)$. Recall that $\mathcal{Y}_{K^{p}} \Gamma\left(p^{n}\right)$ parametrises elliptic curves with certain level structures away from $p$ and a trivialisation of its $p^{n}$-torsion points. In particular, the Weil pairing induces a trivialisation of $\mu_{p^{n}}(C)$. This defines a map $\mathcal{Y}_{K^{p} \Gamma\left(p^{n}\right)} \rightarrow \operatorname{Isom}\left(\mathbb{Z} / p^{n}, \mu_{p^{n}}(C)\right)$, which extends to its compactification $\mathcal{X}_{K^{p}} \Gamma\left(p^{n}\right) \rightarrow \operatorname{Isom}\left(\mathbb{Z} / p^{n}, \mu_{p^{n}}(C)\right)$. Classically, this map can be obtained by looking at the connected components. Taking inverse limits, we obtain $\mathcal{X}_{K^{p}} \rightarrow \operatorname{Isom}\left(\mathbb{Z}_{p}, \mathbb{Z}_{p}(1)\right)$, where $\operatorname{Isom}\left(\mathbb{Z}_{p}, \mathbb{Z}_{p}(1)\right)$ is identified as $\operatorname{Spa}\left(\mathscr{C}\left(\operatorname{Isom}\left(\mathbb{Z}_{p}, \mathbb{Z}_{p}(1)\right), C\right), \mathscr{C}\left(\operatorname{Isom}\left(\mathbb{Z}_{p}, \mathbb{Z}_{p}(1)\right), \mathcal{O}_{C}\right)\right)$. Recall that we have chosen a basis of $\mathbb{Z}_{p}(1)$ before Theorem 4.2.4; that is, an isomorphism $\operatorname{Isom}\left(\mathbb{Z}_{p}, \mathbb{Z}_{p}(1)\right) \cong \mathbb{Z}_{p}^{\times}$. Then $\mathcal{X}_{K^{p}} \rightarrow \operatorname{Isom}\left(\mathbb{Z}_{p}, \mathbb{Z}_{p}(1)\right) \cong \mathbb{Z}_{p}^{\times}$defines a function

$$
t \in H^{0}\left(\mathcal{X}_{K^{p}}, \mathcal{O}_{\mathcal{X}_{K} p}\right)
$$

which is well-defined up to $\mathbb{Z}_{p}^{\times}$. See also (4.2) of [Eme06b] for another description. We remark that $G_{\mathbb{Q}_{p}}$ acts on $t$ via the cyclotomic character because it is essentially an element of $\mathbb{Z}_{p}(1)$. The group $\mathrm{GL}_{2}\left(\mathbb{Q}_{p}\right)$ acts on $t$ via $\varepsilon_{p}$ o det, where $\varepsilon_{p}: \mathbb{Q}_{p}^{\times} \rightarrow \mathbb{Z}_{p}^{\times}$sends $x$ to $x|x|$; hence, the action of $\mathfrak{g}=\mathfrak{g l}_{2}\left(\mathbb{Q}_{p}\right)$ on $t$ is the trace map

$$
\left(\begin{array}{ll}
a & b \\
c & d
\end{array}\right) \cdot t=(a+d) t,\left(\begin{array}{ll}
a & b \\
c & d
\end{array}\right) \in \mathfrak{g} .
$$

If we take the direct limit over all tame levels $K^{p}: \tilde{H}^{0}(C):=\lim _{\longrightarrow} H^{p} H^{0}\left(\mathcal{X}_{K^{p}}, \mathcal{O}_{\mathcal{X}_{K} p}\right)$, then $\mathrm{GL}_{2}\left(\mathbb{A}_{f}\right)$ acts naturally on $\tilde{H}^{0}(C)$ and acts on $t$ via $\varepsilon \circ$ det. See Notation in the beginning of this article for the definition of $\varepsilon$. Using this observation, it is easy to figure out how Hecke operators away from $p$ change when multiplying by a power of $t$.

For $n \geq 1$, we fix $t_{n} \in H^{0}\left(\mathcal{X}_{K^{p} \Gamma\left(p^{n}\right)}, \mathcal{O}_{\mathcal{X}_{K} p_{\Gamma\left(p^{n}\right)}}\right)$ so that $\left\|t-t_{n}\right\| \leq p^{-n}$.

4.3.2 (A consequence of $\theta=0$ ). We introduced a basis $\mathfrak{B}$ of affinoid open subsets of $\mathscr{F} \ell$ in Theorem 4.1.7. Fix $U \in \mathfrak{B}$ from now on. Then by our construction, $V_{\infty}:=\pi_{\mathrm{HT}}^{-1}(U)$ is affinoid perfectoid and is the preimage of an affinoid subset $V_{K_{p}} \subset \mathcal{X}_{K^{p} K_{p}}$. Recall that we have fake-Hasse invariants $e_{1}, e_{2} \in H^{0}\left(\mathcal{X}_{K^{p}}, \omega_{K^{p}}\right)$. Throughout this subsection, we assume

$\circ e_{1}$ generates $H^{0}\left(V_{\infty}, \omega_{K^{p}}\right)$. 
In our later computations, we will always assume one of $e_{1}, e_{2}$ generates $H^{0}\left(V_{\infty}, \omega_{K^{p}}\right)$. When $e_{2}$ generates $H^{0}\left(V_{\infty}, \omega_{K} p\right)$, one can use the action of $\left(\begin{array}{ll}0 & 1 \\ 1 & 0\end{array}\right)$ to reduce to the above case. When $e_{1}$ is a basis, we can take $e=e_{1}$ in Theorem 4.2.4. Hence, up to a unit, $\theta$ on $V_{\infty}$ is

$$
\left(\begin{array}{cc}
x & x^{2} \\
-1 & -x
\end{array}\right) \in \mathfrak{g l}_{2}\left(\mathcal{O}_{K^{p}}(U)\right)
$$

where $x:=\frac{e_{2}}{e_{1}} \in \mathcal{O}_{\mathscr{F} \mathcal{l}}(U)$, a standard coordinate on $\mathscr{F} \ell=\mathbb{P}^{1}$. In particular, we have the following important lemma.

Lemma 4.3.3. Suppose $f \in \mathcal{O}_{K^{p}}^{\mathrm{la}}(U)$ is annihilated by $\mathfrak{b}=\left\{\left(\begin{array}{l}* * \\ 0 *\end{array}\right)\right\} \subset \mathfrak{g l}_{2}\left(\mathbb{Q}_{p}\right)$. Then $f$ is annihilated by $\mathfrak{g l}_{2}\left(\mathbb{Q}_{p}\right)$; that is, $f$ is fixed by an open subgroup of $\mathrm{GL}_{2}\left(\mathbb{Q}_{p}\right)$.

Proof. By Theorem 3.1.2, $\theta(f)=0$. But $\mathfrak{b} \cdot f=0$. Hence, $\theta(f)=\left(\begin{array}{cc}0 & 0 \\ -1 & 0\end{array}\right) \cdot f=0$. Thus, $\mathfrak{g l}_{2}\left(\mathbb{Q}_{p}\right)$. $f=0$.

Remark 4.3.4. Here is a sketch of another proof without using $\theta$. By using the action of $\left(\begin{array}{ll}p & 0 \\ 0 & 1\end{array}\right)$, one may assume $V_{\infty}$ is a rational open subset of some $\epsilon$-neighbourhood of the anticanonical locus $\mathcal{X}_{\Gamma\left(p^{*}\right)}^{*}(\epsilon)_{a}$ for some $\epsilon \in(0,1 / 2)$. See Chapter III of [Sch15] for notation here. Since $f$ is annihilated by $\mathfrak{b}$, it is fixed by an open subgroup $\Gamma_{0}^{\prime}$ of the upper-triangular Borel in $\mathrm{GL}_{2}\left(\mathbb{Q}_{p}\right)$. We may assume $\Gamma_{0}^{\prime} \subset K_{p}$ and denote by $V_{\Gamma_{0}^{\prime}}$ the preimage of $V_{K_{p}}$ in $\mathcal{X}_{\Gamma_{0}^{\prime}}^{*}(\epsilon)_{a}$. Then $f$ comes from a section in $\mathcal{O}_{\mathcal{X}_{\Gamma_{0}^{\prime}}^{*}(\epsilon)_{a}}\left(V_{\Gamma_{0}^{\prime}}\right)$. The key point here is that by Corollary III.2.23 of [Sch15], there exist Tate's normalised traces from $\mathcal{O}_{\mathcal{X}_{\Gamma_{0}^{\prime}}^{*}(\epsilon)_{a}}$ to sections on finite levels. Hence, a standard argument shows that $f$ comes from some finite level by analyticity; cf. the proof of Lemma 3.2.5 or [BC16, Théorème 3.2]. Thus, $f$ is fixed by some open subgroup of $\mathrm{GL}_{2}\left(\mathbb{Q}_{p}\right)$.

4.3.5. Now we can give an explicit description of $\mathcal{O}_{K^{p}}^{\text {la }}(U)$, following Subsection 4.2, 5.2 of [BC16]. The basic idea is to find 'power series expansions along $e_{1}, e_{2}, t$ '. Note that the actions of $K_{p}$ on these elements are analytic. Our setup is as follows. Fix a compact open subgroup $G_{0}$ of $K_{p}$ equipped with an integer-valued, saturated $p$-valuation. For example, one can take $1+p^{m} M_{2}\left(\mathbb{Z}_{p}\right)$ for sufficiently large $m$. Then we have $G_{n}=G_{0}^{p^{n}}$ as in Section 2.1.1. Let $V_{G_{n}} \subset \mathcal{X}_{K^{p} G_{n}}$ be the preimage of $V_{K_{p}}$. By our choice of $U$ (cf. Theorem 4.1.7), the direct limit $\lim _{\longrightarrow} H^{0}\left(V_{G_{n}}, \mathcal{O}_{\mathcal{X}_{K}{ }^{{ }_{G}}{ }_{n}}\right) \rightarrow H^{0}\left(V_{\infty}, \mathcal{O}_{\mathcal{X}_{K} p}\right)$ has dense image. Hence, for any $n \geq 0$, we can find (cf. Section 2.1.4 and Lemma 2.1.5)

$\circ$ an integer $r(n)>r(n-1)>0$;

$\circ x_{n} \in H^{0}\left(V_{G_{r(n)}}, \mathcal{O}_{\mathcal{X}_{K} p_{G_{r(n)}}}\right)$ such that $\left\|x-x_{n}\right\|_{G_{r(n)}}=\left\|x-x_{n}\right\| \leq p^{-n}$ in $H^{0}\left(V_{\infty}, \mathcal{O}_{\mathcal{X}_{K} p}\right)$.

As in Section 4.3.1, we can find (after replacing $r(n)$ by a larger number if necessary)

$\circ t_{n} \in \mathcal{O}_{\mathcal{X}_{K^{p}} G_{r(n)}}\left(\mathcal{X}_{K^{p} G_{r(n)}}\right)$ such that $\left\|t-t_{n}\right\|_{G_{r(n)}}=\left\|t-t_{n}\right\| \leq p^{-n}$ in $H^{0}\left(\mathcal{X}_{K^{p}}, \mathcal{O}_{\mathcal{X}_{K} p}\right)$.

We can define a norm on $H^{0}\left(V_{\infty}, \omega_{K^{p}}\right)$ by identifying it with $H^{0}\left(V_{\infty}, \mathcal{O}_{\mathcal{X}_{K}}\right)$ using $e_{1}$. It is easy to see $\lim _{\longrightarrow n} H^{0}\left(V_{G_{n}}, \omega\right) \rightarrow H^{0}\left(V_{\infty}, \omega_{K} p\right)$ also has dense image. Here, by abuse of notation, $\omega$ denotes 'the $\omega$ $\overrightarrow{\text { on }} \mathcal{X}_{K^{p} K_{p}}$. Hence, we can also find (after enlarging $r(n)$ if necessary)

$\circ e_{1, n} \in H^{0}\left(V_{G_{r(n)}}, \omega\right)$ such that $\left\|e_{1}-e_{1, n}\right\|_{G_{r(n)}}=\left\|e_{1}-e_{1, n}\right\| \leq p^{-n}$ in $H^{0}\left(V_{\infty}, \omega_{K^{p}}\right)$.

Note that $e_{1, n}$ is also a basis of $H^{0}\left(V_{\infty}, \omega_{K^{p}}\right)$, if $n>0$. 
As before, we denote by $\mathfrak{b}=\left\{\left(\begin{array}{l}* * \\ 0 *\end{array}\right)\right\} \subset \mathfrak{g l}_{2}\left(\mathbb{Q}_{p}\right)$ the Lie algebra of the upper-triangular Borel subgroup and by $\mathfrak{n}$ its nilpotent subalgebra. Let

$$
u^{+}=\left(\begin{array}{ll}
0 & 1 \\
0 & 0
\end{array}\right), h=\left(\begin{array}{cc}
1 & 0 \\
0 & -1
\end{array}\right), z=\left(\begin{array}{ll}
1 & 0 \\
0 & 1
\end{array}\right)
$$

be a basis of $\mathfrak{b}$. Then $u^{+} \cdot x=u^{+} \cdot \frac{e_{2}}{e_{1}}=1$ and $h \cdot e_{1}=e_{1}$ and $z \cdot t=2 t$.

4.3.6 (Expansion along $\mathfrak{n}$ ). Fix $f \in \mathcal{O}_{K^{p}}^{\text {la }}(U)$. We are going to write $f$ as a power series. Suppose $f \in \mathcal{O}_{K^{p}}(U)^{G_{m}-\text { an }}$. By Lemma 2.1.8, there is a constant $C_{1}$ such that $\left\|u^{+} \cdot s\right\|_{G_{m}} \leq p^{C_{1}}\|s\|_{G_{m}}$, for any $s \in \mathcal{O}_{K^{p}}(U)^{G_{m} \text { an }}$. Choose $n^{\prime \prime} \geq \max \left(C_{1}+1 /(p-1)+1, m\right)$. Then

$$
\left\|\left(x-x_{n^{\prime \prime}}\right)^{l} \frac{\left(u^{+}\right)^{l} \cdot s}{l !}\right\|_{G_{r\left(n^{\prime \prime}\right)}} \leq p^{-l}\|s\|_{G_{r(m)}}
$$

for any $l \geq 0$ and $s \in \mathcal{O}_{K^{p}}(U)^{G_{m}-\text { an }}$ by a simple calculation. Hence,

$$
D_{x}(s):=\sum_{l=0}^{+\infty}(-1)^{l}\left(x-x_{n^{\prime \prime}}\right)^{l} \frac{\left(u^{+}\right)^{l} \cdot s}{l !}
$$

defines a bounded map $D_{x}: \mathcal{O}_{K^{p}}(U)^{G_{m}-\text { an }} \rightarrow \mathcal{O}_{K^{p}}(U)^{G_{r\left(n^{\prime \prime}\right)}-\text { an }}$ with norm $\leq 1$. Moreover, since $u^{+} \cdot\left(x-x_{n^{\prime \prime}}\right)=1$, one checks easily $u^{+} \circ D_{x}=0$. Let

$$
a_{i}:=D_{x}\left(\frac{\left(u^{+}\right)^{i} \cdot f}{i !}\right)=\sum_{l=0}^{+\infty}(-1)^{l}\left(x-x_{n^{\prime \prime}}\right)^{l} \frac{\left(u^{+}\right)^{l+i} \cdot f}{l ! i !} \in \mathcal{O}_{K^{p}}(U)^{G_{r\left(n^{\prime \prime}\right)}-\mathrm{an}} .
$$

Its norm $\left\|a_{i}\right\|_{G_{r\left(n^{\prime \prime}\right)}} \leq\left\|\frac{\left(u^{+}\right)^{i} \cdot f}{i !}\right\|_{G_{m}} \leq p^{\left(n^{\prime \prime}-1\right) i}\|f\|_{G_{m}}$. Thus, $\sum_{i \geq 0} a_{i}\left(x-x_{n^{\prime \prime}}\right)^{i}$ is convergent in $\mathcal{O}_{K^{p}}(U)^{G_{r\left(n^{\prime \prime}\right)}-\text { an }}$ and a direct computation shows (using $\sum_{i=0}^{l}(-1)^{i} \frac{1}{i !(l-i) !}=0$ if $l \geq 1$ ) that in fact

$$
f=\sum_{i=0}^{+\infty} a_{i}\left(x-x_{n^{\prime \prime}}\right)^{i}
$$

in $\mathcal{O}_{K^{p}}(U)^{G_{r\left(n^{\prime \prime}\right)}-\text { an }}$. Note that $u^{+} \cdot a_{i}=0$ for any $i$ by our construction.

4.3.7 (Expansion along $h$ ). Next we write $a_{i}$ as a power series. Note that $\left\|e_{1}-e_{1, l}\right\|_{G_{r(l)}}=\left\|e_{1}-e_{1, l}\right\| \leq$ $p^{-l}$. Hence, $\left\|e_{1, l}\right\|_{G_{r(l)}}=\left\|e_{1}\right\|_{G_{r(l)}}=1$. For $j \geq 1$, the series

$$
\log \left(\frac{e_{1}}{e_{1, l}}\right)=\log \left(1+\frac{e_{1}-e_{1, l}}{e_{1, l}}\right):=-\sum_{j=1}^{+\infty}(-1)^{j} \frac{1}{j}\left(\frac{e_{1}}{e_{1, l}}-1\right)^{j}
$$

converges in $\mathcal{O}_{K^{p}}(U)^{G_{r(l)}-\text { an }}$ and has norm $\left\|\log \left(\frac{e_{1}}{e_{1, l}}\right)\right\|_{G_{r(l)}} \leq p^{-l}$. Moreover, since $u^{+} \cdot e_{1}=0, h \cdot e_{1}=$ $e_{1}$, it follows that $u^{+} \cdot \log \left(\frac{e_{1}}{e_{1, l}}\right)=0, h \cdot \log \left(\frac{e_{1}}{e_{1, l}}\right)=1$. Hence, we can repeat the previous process with $u^{+}, x-x_{n^{\prime \prime}}$ replaced by $h, \log \left(\frac{e_{1}}{e_{1, n^{\prime}}}\right)$ : by Lemma 2.1 .8 , there is a constant $C_{2}$ such that $\|h \cdot s\|_{G_{r\left(n^{\prime \prime}\right)}} \leq$

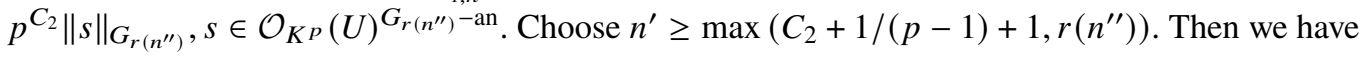

$$
a_{i}=\sum_{j=0}^{+\infty} b_{i, j}\left(\log \left(\frac{e_{1}}{e_{1, n^{\prime}}}\right)\right)^{j}
$$


in $\mathcal{O}_{K^{p}}(U)^{G_{r\left(n^{\prime}\right)}-\text { an }}$, where

$$
b_{i, j}=\sum_{l=0}^{+\infty}(-1)^{l}\left(\log \left(\frac{e_{1}}{e_{1, n^{\prime}}}\right)\right)^{l} \frac{h^{l+j} \cdot a_{i}}{l ! j !}
$$

is convergent in $\mathcal{O}_{K^{p}}(U)^{G_{r\left(n^{\prime}\right)}-\text { an }}$ and has norm $\left\|b_{i, j}\right\|_{G_{r\left(n^{\prime}\right)}} \leq p^{\left(n^{\prime}-1\right) j+\left(n^{\prime \prime}-1\right) i}\|f\|_{G_{m}}$. Also, since $\left[h, u^{+}\right]=2 u^{+}$and $u^{+} \cdot a_{i}=0$, we have

$$
u^{+} \cdot b_{i, j}=h \cdot b_{i, j}=0
$$

4.3.8 (Expansion along $z$ ). Finally, we can expand $b_{i, j}$ using $z$. This is almost the same as in Section 4.3.7. Note that $t$ is invertible with norm 1 ; hence, $t_{l}$ is also invertible. Thus, $\log \left(\frac{t}{t_{l}}\right)$ converges in $\mathcal{O}_{K^{p}}(U)^{G_{r(l)}-\text { an }}$ with norm $\leq p^{-l}$. It satisfies $u^{+} \cdot \log \left(\frac{t}{t_{l}}\right)=h \cdot \log \left(\frac{t}{t_{l}}\right)=0$ and $z \cdot \log \left(\frac{t}{t_{l}}\right)=2$. Then for any sufficiently large integer $n \geq r\left(n^{\prime}\right)$, we have

$$
b_{i, j}=\sum_{k=0}^{+\infty} c_{i, j, k}\left(\log \left(\frac{t}{t_{n}}\right)\right)^{k}
$$

converges in $\mathcal{O}_{K^{p}}(U)^{G_{r(n)} \text {-an }}$, where

$$
c_{i, j, k}=\frac{1}{2^{k}} \sum_{l=0}^{+\infty}(-1)^{l}\left(\frac{1}{2} \log \left(\frac{t}{t_{n}}\right)\right)^{l} \frac{z^{l+k} \cdot b_{i, j}}{l ! k !}
$$

and has norm

$$
\left\|c_{i, j, k}\right\|_{G_{r(n)}} \leq p^{(n-1) k+\left(n^{\prime}-1\right) j+\left(n^{\prime \prime}-1\right) i}\|f\|_{G_{m}} .
$$

Since $z$ commutes with $u^{+}, h$, it follows that

$$
u^{+} \cdot c_{i, j, k}=h \cdot c_{i, j, k}=z \cdot c_{i, j, k}=0
$$

that is, $c_{i, j, k}$ is annihilated by $\mathfrak{b}$. By Lemma 4.3.3, this implies $c_{i, j, k}$ is fixed $G_{r(n)}$. Therefore,

$$
c_{i, j, k} \in H^{0}\left(V_{G_{r(n)}}, \mathcal{O}_{\mathcal{X}_{K^{p}} G_{r(n)}}\right) .
$$

In particular, $\left\|c_{i, j, k}\right\|_{G_{r(n)}}=\left\|c_{i, j, k}\right\|$.

Theorem 4.3.9. Keep the notation in Section 4.3.5. For any $f \in \mathcal{O}_{K^{p}}^{\text {la }}(U)$ which is $G_{m^{-a n a l y t i c} \text {, there }}$ exist an integer $N=N(f)$, bounded above by some constant only depending on $m$, and unique elements $c_{i, j, k}^{(n)}(f) \in H^{0}\left(V_{G_{r(n)}}, \mathcal{O}_{\mathcal{X}_{K} p_{G_{r(n)}}}\right)$ for $i, j, k \geq 0, n \geq N$ with norm $\left\|c_{i, j, k}^{(n)}(f)\right\| \leq p^{(n-1)(i+j+k)}\|f\|_{G_{m}}$ for which

$$
f=\sum_{i, j, k \geq 0} c_{i, j, k}^{(n)}(f)\left(x-x_{n}\right)^{i}\left(\log \left(\frac{e_{1}}{e_{1, n}}\right)\right)^{j}\left(\log \left(\frac{t}{t_{n}}\right)\right)^{k}
$$

holds in $\mathcal{O}_{K^{p}}(U)^{G_{r(n)}{ }^{-a n}}$. Conversely, given $n>0$ and $\mathrm{c}_{i, j, k} \in H^{0}\left(V_{G_{r(n)}}, \mathcal{O}_{\mathcal{X}_{K^{p}} G_{r(n)}}\right), i, j, k \geq 0$ such that $\left\|\mathrm{c}_{i, j, k}\right\| \leq p^{(n-1)(i+j+k)} C^{\prime}$ holds for a uniform constant $C^{\prime}$,

$$
f^{\prime}:=\sum_{i, j, k \geq 0} \mathrm{c}_{i, j, k}\left(x-x_{n}\right)^{i}\left(\log \left(\frac{e_{1}}{e_{1, n}}\right)\right)^{j}\left(\log \left(\frac{t}{t_{n}}\right)\right)^{k}
$$

defines an element in $\mathcal{O}_{K^{p}}(U)^{G_{r(n)}-\text { an }}$. We can take $N\left(f^{\prime}\right)=n$; hence, $c_{i, j, k}^{(n)}\left(f^{\prime}\right)=\mathrm{c}_{i, j, k}$. 
Proof. We have written $f=\sum_{i, j, k \geq 0} c_{i, j, k}\left(x-x_{n^{\prime \prime}}\right)^{i}\left(\log \left(\frac{e_{1}}{e_{1, n^{\prime}}}\right)\right)^{j}\left(\log \left(\frac{t}{t_{n}}\right)\right)^{k}$ with $\left\|c_{i, j, k}\right\|_{G_{r(n)}} \leq$ $p^{(n-1) k+\left(n^{\prime}-1\right) j+\left(n^{\prime \prime}-1\right) i}\|f\|_{G_{m}}$. The rest is to change the coordinates from $x-x_{n^{\prime \prime}}$ to $x-x_{n}$ and from $\log \left(\frac{e_{1}}{e_{1, n^{\prime}}}\right)$ to $\log \left(\frac{e_{1}}{e_{1, n}}\right)$. By our construction, both $\left(x-x_{n}\right)-\left(x-x_{n^{\prime \prime}}\right), \log \left(\frac{e_{1}}{e_{1, n}}\right)-\log \left(\frac{e_{1}}{e_{1, n^{\prime}}}\right) \in$ $H^{0}\left(V_{G_{r(n)}}, \mathcal{O}_{\mathcal{X}_{K} p_{G_{r(n)}}}\right)$ and

$$
\left\|\left(x-x_{n}\right)-\left(x-x_{n^{\prime \prime}}\right)\right\| \leq p^{-n^{\prime \prime}},\left\|\log \left(\frac{e_{1}}{e_{1, n}}\right)-\log \left(\frac{e_{1}}{e_{1, n^{\prime}}}\right)\right\| \leq p^{-n^{\prime}} .
$$

Hence, if we write

$$
\sum_{i, j, k \geq 0} c_{i, j, k}\left(x-x_{n^{\prime \prime}}\right)^{i}\left(\log \left(\frac{e_{1}}{e_{1, n^{\prime}}}\right)\right)^{j}\left(\log \left(\frac{t}{t_{n}}\right)\right)^{k}=\sum_{i, j, k \geq 0} c_{i, j, k}^{(n)}(f)\left(x-x_{n}\right)^{i}\left(\log \left(\frac{e_{1}}{e_{1, n}}\right)\right)^{j}\left(\log \left(\frac{t}{t_{n}}\right)\right)^{k}
$$

for some $c_{i, j, k}^{(n)}(f) \in H^{0}\left(V_{G_{r(n)}}, \mathcal{O}_{\mathcal{X}_{K}{ }^{p_{r(n)}}}\right)$, a simple computation gives

$$
\left\|c_{i, j, k}^{(n)}(f)\right\|_{G_{r(n)}} \leq p^{(n-1) k+\left(n^{\prime}-1\right) j+\left(n^{\prime \prime}-1\right) i}\|f\|_{G_{m}} \leq p^{(n-1)(i+j+k)}\|f\|_{G_{m}}
$$

as $n \geq n^{\prime} \geq n^{\prime \prime}$. For the uniqueness and the converse part, using the bounds on $c_{i, j, k}^{(n)}(f)$, we can repeat our construction (with $n^{\prime \prime}=n^{\prime}=n$ this time) and recover $c_{i, j, k}^{(n)}(f)$ from $f$. We omit the details here.

Definition 4.3.10. Keep the notation in Section 4.3.5. For any $n>0$, we define $\mathcal{O}^{n}(U)\left\{x, e_{1}, t\right\} \subset$ $\mathcal{O}_{K^{p}}^{\text {la }}(U)$ to be the subset of $f$, which can be written as

$$
f=\sum_{i, j, k \geq 0} c_{i, j, k}\left(x-x_{n}\right)^{i}\left(\log \left(\frac{e_{1}}{e_{1, n}}\right)\right)^{j}\left(\log \left(\frac{t}{t_{n}}\right)\right)^{k}
$$

with $c_{i, j, k} \in H^{0}\left(V_{G_{r(n)}}, \mathcal{O}_{\mathcal{X}_{K}{ }^{G_{r(n)}}}\right), i, j, k \geq 0$ such that $\left\|c_{i, j, k}\right\| \leq p^{(n-1)(i+j+k)} C^{\prime}$ holds for some uniform constant $C^{\prime}$. It is a Banach algebra over $C$ with norm

$$
\|f\|_{n}:=\sup _{i, j, k \geq 0}\left\|c_{i, j, k}^{(n)}(f) p^{(n-1)(i+j+k)}\right\| .
$$

Let $\mathcal{O}^{n}(U)^{+}\left\{x, e_{1}, t\right\}$ be its open unit ball. By Theorem 4.3.9, sending $f$ to $c_{i, j, k}^{(n)}(f) p^{(n-1)(i+j+k)}$ induces an isomorphism of topological $\mathbb{Z}_{p}$-modules

$$
\mathcal{O}^{n}(U)^{+}\left\{x, e_{1}, t\right\} \cong \prod_{i, j, k \geq 0} H^{0}\left(V_{G_{r(n)}}, \mathcal{O}_{\mathcal{X}_{K}{ }^{G_{r}(n)}}\right) .
$$

Remark 4.3.11. It is clear from the proof of Theorem 4.3.9 that $\mathcal{O}^{n}(U)\left\{x, e_{1}, t\right\}$ is independent of the choice of $x_{n}, e_{1, n}, t_{n}$.

Remark 4.3.12. For any $m>0$, by Theorem 4.3.9, we can find $n$ such that there are continuous embeddings (of Banach spaces)

$$
\mathcal{O}_{K^{p}}(U)^{G_{m}-\mathrm{an}} \rightarrow \mathcal{O}^{n}(U)\left\{x, e_{1}, t\right\} \rightarrow \mathcal{O}_{K^{p}}(U)^{G_{r(n)} \text {-an }} .
$$

Hence, $\lim _{\longrightarrow} \mathcal{O}_{K^{p}}(U)^{G_{n}-\text { an }} \cong \lim _{\longrightarrow n} \mathcal{O}^{n}(U)\left\{x, e_{1}, t\right\}$ as topological spaces. It will be clear later that $\mathcal{O}^{n}(U)\left\{x, e_{1}, t\right\}$ behaves a lot better in applications.

4.3.13. Theorem 4.3.9 can be rephrased sheaf-theoretically. Recall some construction in the paragraph above Corollary 3.6.10. We denote by $\mathcal{O}_{r(n)}^{+}$the pushforward of $\mathcal{O}_{V_{G_{r(n)}}^{+}}$from $V_{G_{r(n)}}$ to $V_{K_{p}}$, by $\widetilde{\mathcal{O}}$ the 
pushforward of $\mathcal{O}_{V_{\infty}}$ from $V_{\infty}$ to $V_{K_{p}}$, by $\widetilde{\mathcal{O}}^{n} \subset \widetilde{\mathcal{O}}$ the subsheaf of $G_{n}$-analytic sections and by $\widetilde{\mathcal{O}}^{\text {la }} \subset \widetilde{\mathcal{O}}$ the subsheaf of $K_{p}$-locally analytic sections. For each $n>0$, we can define a map

$$
\phi_{n}^{+}: \prod_{(i, j, k) \in \mathbb{N}^{3}} \mathcal{O}_{r(n)}^{+} \rightarrow \widetilde{\mathcal{O}}^{\text {la }}
$$

sending $\left(a_{i, j, k}\right) \in \prod_{i, j, k \geq 0} \mathcal{O}_{r(n)}^{+}(W)$ to

$$
\sum_{i, j, k \geq 0} p^{-(n-1)(i+j+k)} a_{i, j, k}\left(x-x_{n}\right)^{i}\left(\log \left(\frac{e_{1}}{e_{1, n}}\right)\right)^{j}\left(\log \left(\frac{t}{t_{n}}\right)\right)^{k} \in \widetilde{\mathcal{O}}^{\mathrm{la}}(W),
$$

for any open set $W$ of $V_{K_{p}}$. Let $\phi_{n}:\left(\prod_{(i, j, k) \in \mathbb{N}^{3}} \mathcal{O}_{r(n)}^{+}\right) \otimes_{\mathbb{Z}_{p}} \mathbb{Q}_{p} \rightarrow \widetilde{\mathcal{O}}^{\text {la }}$ be $\phi_{n}^{+} \otimes \mathbb{Q}_{p}$. Then Theorem 4.3.9 implies that $\phi_{n}$ is an isomorphism onto its image $\operatorname{im}\left(\phi_{n}\right)$ and for any $m>0$, we have $\widetilde{\mathcal{O}}^{m} \subset$ $\operatorname{im}\left(\phi_{n}\right) \subset \widetilde{\mathcal{O}}^{r(n)}$ for some $n$. A direct consequence is the following.

Lemma 4.3.14. Let $\mathfrak{U}$ be a finite cover of $V_{K^{p}}$ by rational open subsets. We have the following assertions for Čech cohomology with respect to $\mathfrak{U}$.

(1) $\check{H}^{i}\left(\mathfrak{U}, \prod_{(i, j, k) \in \mathbb{N}^{3}} \mathcal{O}_{r(n)}^{+}\right) \otimes_{\mathbb{Z}_{p}} \mathbb{Q}_{p}=0, i \geq 1$.

(2) The direct system $\left\{\breve{H}^{i}\left(\mathfrak{U}, \widetilde{\mathcal{O}}^{n}\right)\right\}_{n}$ is essentially zero for any $i \geq 1$.

Proof. Let $C^{\bullet}\left(\mathfrak{U}, \mathcal{O}_{r(n)}^{+}\right)$be the Čech complex for $\mathcal{O}_{r(n)}^{+}$with respect to $\mathfrak{U}$. Note that if we denote by $\mathfrak{U}^{\prime}$ the pullback of $\mathfrak{U}$ to $V_{G_{r(n)}}$, then $C^{\bullet}\left(\mathfrak{U}, \mathcal{O}_{r(n)}^{+}\right)$is nothing but the Čech complex for $\mathcal{O}_{V_{G_{r(n)}}^{+}}$ with respect to $\mathfrak{U}^{\prime}$. Hence, Tate's acyclicity result implies that $H^{i}\left(C^{\bullet}\left(\mathfrak{U}, \mathcal{O}_{r(n)}^{+}\right)\right) \otimes_{\mathbb{Z}_{p}} \mathbb{Q}_{p}=0, i \geq 1$. Therefore, $H^{i}\left(C^{\bullet}\left(\mathfrak{U}, \mathcal{O}_{r(n)}^{+}\right)\right), i \geq 1$ is annihilated by some $p^{k}$ by open mapping theorem. From this, we get $H^{i}\left(C^{\bullet}\left(\mathfrak{H}, \prod_{(i, j, k) \in \mathbb{N}^{3}} \mathcal{O}_{r(n)}^{+}\right)\right) \otimes_{\mathbb{Z}_{p}} \mathbb{Q}_{p}=0, i \geq 1$, which is exactly what we want.

The second part is a direct consequence of the first one and the inclusion $\widetilde{\mathcal{O}}^{m} \subset \operatorname{im}\left(\phi_{n}\right) \subset \widetilde{\mathcal{O}}^{r(n)}$.

Since $\mathcal{O}_{K^{p}}(U)$ is a Banach space representation of $K_{p}$, we can talk about its (strongly) $\mathfrak{Q A}$-acyclicity (with respect to $K_{p}$ ). See Subsection 2.2 for more details.

Proposition 4.3.15. $\mathcal{O}_{K^{p}}(U)$ is strongly $\mathfrak{Q A}$-acyclic for any $U \in \mathfrak{B}$.

Proof. By Lemma 5.2 and its proof of [Sch13a], we can find a cover of $V_{K_{p}}$ by finitely many rational subsets $U_{1}, \cdots, U_{m}$ such that each $U_{i}$ is small in the sense of Section 3.1.1 with $S=$ \{cusp in $U_{i}$ \}. By Corollary 4.2.3, the preimage of $U_{i}$ in $V_{\infty}$ is a locally analytic covering of $U_{i}$. The proposition now follows from Corollary 3.6.10 and Lemma 4.3.14.

\subsection{Cohomology of $\mathcal{O}_{K^{p}}^{\mathrm{la}}$ and completed cohomology}

In this subsection, we compare the coherent cohomology of $\mathcal{O}_{K^{p}}^{\text {la }}\left(\right.$ on $\mathscr{F} \ell$ ) and $\mathcal{O}_{\mathcal{X}_{K^{p}}}$ (on $\mathcal{X}_{K^{p}}$ ). By Scholze's result [Sch15], the latter one is closely related to the completed cohomology of modular curves introduced earlier by Emerton [Eme06b]. The main result is that the cohomology of $\mathcal{O}_{K^{p}}^{\text {la }}$ is more or less the subspace of locally analytic vectors in completed cohomology.

4.4.1. First recall the construction of completed cohomology. See, for example, [Eme06b, CE12]. For a tame level $K^{p} \subset \mathrm{GL}_{2}\left(\mathbb{A}_{f}^{p}\right)$, let

$$
\tilde{H}^{i}\left(K^{p}, \mathbb{Z} / p^{n}\right):=\lim _{K_{p} \subset \mathrm{GL}_{2}\left(\mathbb{Q}_{p}\right)} H^{i}\left(Y_{K^{p}} K_{p}(\mathbb{C}), \mathbb{Z} / p^{n}\right) .
$$


Since $Y_{K^{p} K_{p}}(\mathbb{C})$ is affine, $\tilde{H}^{i}=0, i \geq 2$. Note that $\tilde{H}^{i}\left(K^{p}, \mathbb{Z} / p^{n}\right)$ can also be defined using the compactified modular curves; that is, the natural restriction map

$$
\underset{K_{p}}{\lim } H^{i}\left(X_{K^{p}} K_{p}(\mathbb{C}), \mathbb{Z} / p^{n}\right) \rightarrow \underset{K_{p}}{\lim } H^{i}\left(Y_{K^{p}} K_{p}(\mathbb{C}), \mathbb{Z} / p^{n}\right)
$$

is an isomorphism. This is clear when $i=0$. When $i=2$, both sides are zero. When $i=1$, the cokernel of above comes from cohomology of top degree around each cusp, which vanishes as the ramification degree of each cusp is divisible by arbitrary power of $p$.

The completed cohomology of tame level $K^{p}$ is defined as

$$
\tilde{H}^{i}\left(K^{p}, \mathbb{Z}_{p}\right):={\underset{\lim }{n}}_{n} \tilde{H}^{i}\left(K^{p}, \mathbb{Z} / p^{n}\right)
$$

It has a natural admissible continuous action of $\mathrm{GL}_{2}\left(\mathbb{Q}_{p}\right)$; that is, $\tilde{H}^{i}\left(K^{p}, \mathbb{Z}_{p}\right) / p$ is a smooth admissible representation of $\mathrm{GL}_{2}\left(\mathbb{Q}_{p}\right)$ over $\mathbb{F}_{p}$; cf. [CE12, Theorem 1.16]. As a consequence, $\tilde{H}^{i}\left(K^{p}, \mathbb{Z}_{p}\right)$ has bounded $p$-torsion; that is, $p$-power torsion classes in $\tilde{H}^{i}\left(K^{p}, \mathbb{Z}_{p}\right)$ have bounded exponent.

The following result (essentially due to Scholze) relates completed cohomology and the cohomology of $\mathcal{O}_{\mathcal{X}_{K} p}^{+}$. Here we say a map is Hecke-equivariant if it commutes with Hecke operators away from $p$.

Theorem 4.4.2. There is a natural $\mathrm{GL}_{2}\left(\mathbb{Q}_{p}\right)$ and Hecke-equivariant isomorphism of almost $\mathcal{O}_{C^{-}}$ modules

$$
\tilde{H}^{i}\left(K^{p}, \mathbb{Z} / p^{n}\right) \otimes_{\mathbb{Z}_{p} / p^{n}} \mathcal{O}_{C} / p^{n} \cong H^{i}\left(\mathcal{X}_{K^{p}}, \mathcal{O}_{\mathcal{X}_{K} p}^{+} / p^{n}\right),
$$

where the right-hand side is computed using the analytic topology of $\mathcal{X}_{K^{p}}$.

Proof. Basically the same proof of Theorem IV.2.1 of [Sch15] works here: first we may identify $H^{i}\left(X_{K^{p} K_{p}}(\mathbb{C}), \mathbb{Z} / p^{n}\right)$ with $H_{\text {ét }}^{i}\left(\mathcal{X}_{K^{p} K_{p}}, \mathbb{Z} / p^{n}\right)$ by the comparison theorem; then, using the primitive comparison theorem (Theorem 1.3 of [Sch13a]) and taking the direct limit over all $K_{p}$, we obtain the desired almost isomorphism.

Corollary 4.4.3. There is a natural $\mathrm{GL}_{2}\left(\mathbb{Q}_{p}\right)$ and Hecke-equivariant isomorphism of almost $\mathcal{O}_{C^{-}}$ modules

$$
\tilde{H}^{i}\left(K^{p}, \mathbb{Z}_{p}\right) \widehat{\otimes}_{\mathbb{Z}_{p}} \mathcal{O}_{C} \cong H^{i}\left(\mathcal{X}_{K^{p}}, \mathcal{O}_{\mathcal{X}^{p}}^{+}\right)
$$

Proof. Since the higher cohomology of $\mathcal{O}_{\mathcal{X}_{K} p}^{+}$almost vanishes on any affinoid perfectoid open subset (Theorem 1.8.(iv) of [Sch12]), we can compute $H^{i}\left(\mathcal{X}_{K^{p}}, \mathcal{O}_{\mathcal{X}_{K} p}^{+}\right)$and $H^{i}\left(\mathcal{X}_{K^{p}}, \mathcal{O}_{\mathcal{X}_{K} p}^{+} / p^{n}\right)$ by Čech cohomology. Take a finite affinoid perfectoid cover of $\mathcal{X}_{K^{p}}$ and let $M^{\bullet}$ be the Čech complex for $\mathcal{O}_{\mathcal{X}_{K}}^{+}$

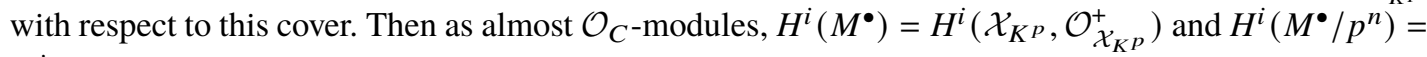
$H^{i}\left(\mathcal{X}_{K^{p}}, \mathcal{O}_{\mathcal{X}_{K} p}^{+} / p^{n}\right)$. In view of the previous theorem, the corollary above is reduced to the following lemma.

Lemma 4.4.4. Let $M^{\bullet}$ be a bounded above chain complex of p-adically complete, p-torsion-free $\mathbb{Z}_{p^{-}}$ modules. Assume that $\lim _{n} H^{i}\left(M^{\bullet} / p^{n}\right)$ has bounded $p$-torsion for any $i$. Then we have natural isomorphisms

$$
H^{i}\left(M^{\bullet}\right) \stackrel{\sim}{\rightarrow} \lim _{n} H^{i}\left(M^{\bullet}\right) / p^{n} \stackrel{\sim}{\rightarrow} \lim _{n} H^{i}\left(M^{\bullet} / p^{n}\right)
$$


Proof. Since $M^{\bullet}$ is $p$-adically complete and $p$-torsion free, we know that $H^{i}\left(M^{\bullet}\right)$ is derived $p$-adically complete. See [Sta20, Tag 091N] for more details. In particular,

$$
\operatorname{Hom}_{\mathbb{Z}_{p}}\left(\mathbb{Q}_{p}, H^{i}\left(M^{\bullet}\right)\right)=0 .
$$

For the reader's convenience, we recall the argument here. Suppose $\operatorname{Hom}_{\mathbb{Z}_{p}}\left(\mathbb{Q}_{p}, H^{i}\left(M^{\bullet}\right)\right) \neq 0$. We can find $x_{n} \in H^{i}\left(M^{\bullet}\right), n=0,1,2, \cdots$ satisfying $p x_{n}=x_{n-1}$ and $x_{0} \neq 0$. Let $\tilde{x}_{n} \in M^{i}$ be a lift of $x_{n}$. Then there exist $y_{n} \in M^{i-1}$ such that $d y_{n}=\tilde{x}_{n-1}-p \tilde{x}_{n}$. Define $y=y_{1}+p y_{2}+p^{2} y_{3}+\cdots \in M^{i-1}$. One checks easily $d y=\tilde{x}_{0}$. Hence, $x_{0}=0$, a contradiction.

Thus, $\operatorname{Hom}_{\mathbb{Z}_{p}}\left(\mathbb{Q}_{p} / \mathbb{Z}_{p}, H^{i}\left(M^{\bullet}\right)\right)=0$. Now by the universal coefficient theorem, we have

$$
0 \rightarrow H^{i}\left(M^{\bullet}\right) / p^{n} \rightarrow H^{i}\left(M^{\bullet} / p^{n}\right) \rightarrow H^{i+1}\left(M^{\bullet}\right)\left[p^{n}\right] \rightarrow 0
$$

When $n$ varies, the transition map $H^{i+1}\left(M^{\bullet}\right)\left[p^{n+1}\right] \rightarrow H^{i+1}\left(M^{\bullet}\right)\left[p^{n}\right]$ is multiplication by $p$. Hence, $\lim _{n} H^{i+1}\left(M^{\bullet}\right)\left[p^{n}\right]=\operatorname{Hom}_{\mathbb{Z}_{p}}\left(\mathbb{Q}_{p} / \mathbb{Z}_{p}, H^{i}\left(M^{\bullet}\right)\right)=0$. We get ${\underset{\leftarrow}{\longleftarrow}}_{n} H^{i}\left(M^{\bullet}\right) / p^{n} \stackrel{\sim}{\rightarrow}{\underset{\lim }{\longleftarrow}}_{n} H^{i}\left(M^{\bullet} / p^{n}\right)$ $\overleftarrow{\text { by }}^{n}$ passing to the limit over $n$ of the above exact sequence.

It remains to show that $H^{i}\left(M^{\bullet}\right) \rightarrow \lim _{\longleftarrow_{n}} H^{i}\left(M^{\bullet}\right) / p^{n}$ is an isomorphism. This is clearly surjective as $H^{i}\left(M^{\bullet}\right)$ is a quotient of $\operatorname{ker}\left(M^{i} \stackrel{d}{\rightarrow} M^{i+1}\right)$, which is $p$-adically complete. Let $K$ be the kernel of this map. By our assumption, all of the torsion in $\lim _{n} H^{i}\left(M^{\bullet}\right) / p^{n}$ can be annihilated by $p^{k}$ for some $k$. For any $x \in K$, we can find $x^{\prime} \in H^{i}\left(M^{\bullet}\right)$ satisfying $p^{k+1} x^{\prime}=x$. Then $x^{\prime}$ maps to a torsion element in $\lim _{n} H^{i}\left(M^{\bullet}\right) / p^{n}$. Hence, $y=p^{k} x^{\prime} \in K$ and $p y=x$. Therefore, $p K=K$, which implies $K=0$ as $\operatorname{Hom}_{\mathbb{Z}_{p}}\left(\mathbb{Q}_{p}, H^{i}\left(M^{\bullet}\right)\right)=0$.

Remark 4.4.5. In fact, it is well-known that $\tilde{H}^{i}\left(K^{p}, \mathbb{Z}_{p}\right)$ is $p$-torsion free because the $p$-adic étale cohomology of curves has no torsion. Hence, the proof of Corollary 4.4.3 can be greatly simplified in this case. We decide to present this complicated proof here because it works in more general settings.

We write $\tilde{H}^{i}\left(K^{p}, \mathcal{O}_{C}\right)=\tilde{H}^{i}\left(K^{p}, \mathbb{Z}_{p}\right) \widehat{\otimes}_{\mathbb{Z}_{p}} \mathcal{O}_{C}$ and $\tilde{H}^{i}\left(K^{p}, C\right)=\tilde{H}^{i}\left(K^{p}, \mathbb{Z}_{p}\right) \widehat{\otimes}_{\mathbb{Z}_{p}} C$. Then

$$
\tilde{H}^{i}\left(K^{p}, C\right) \cong H^{i}\left(\mathcal{X}_{K^{p}}, \mathcal{O}_{\mathcal{X}_{K} p}\right)
$$

is a $\mathbb{Q}_{p}$-Banach representation of $\mathrm{GL}_{2}\left(\mathbb{Q}_{p}\right)$. Our main result here identifies its subspace of $\mathrm{GL}_{2}\left(\mathbb{Q}_{p}\right)$ locally analytic vectors.

Theorem 4.4.6. For any $i \geq 0$, there are natural $\mathrm{GL}_{2}\left(\mathbb{Q}_{p}\right)$ and Hecke-equivariant isomorphisms

○ $\tilde{H}^{i}\left(K^{p}, C\right) \cong H^{i}\left(\mathscr{F} \ell, \mathcal{O}_{K^{p}}\right)$,

○ $\tilde{H}^{i}\left(K^{p}, C\right)^{\mathrm{la}} \cong H^{i}\left(\mathscr{F} \ell, \mathcal{O}_{K^{p}}^{\mathrm{la}}\right)$.

Proof. First note that all higher direct images $R^{j} \pi_{\mathrm{HT} *} \mathcal{O}_{\mathcal{X}_{K} p}=0, j>0$. One can check this on a basis $\mathfrak{B}$ of open subsets of $\mathscr{F} \ell$ in Theorem 4.1.7 and invoke the acyclicity result on affinoid perfectoid spaces; cf. Theorem 1.8.(iv) of [Sch12]. Hence,

$$
H^{i}\left(\mathcal{X}_{K^{p}}, \mathcal{O}_{\mathcal{X}_{K} p}\right)=H^{i}\left(\mathscr{F} \ell, \pi_{\mathrm{HT} *} \mathcal{O}_{\mathcal{X}_{K} p}\right)=H^{i}\left(\mathscr{F} \ell, \mathcal{O}_{K} p\right)
$$

This proves the first isomorphism in the theorem and shows that $H^{i}\left(\mathscr{F} \ell, \mathcal{O}_{K^{p}}\right)$ can be computed by the Cech complex of a finite cover of $\mathscr{F} \ell$ of open subsets in $\mathfrak{B}$. We claim the same is true for $\mathcal{O}_{K^{p}}^{\text {la }}$; that is,

$$
H^{j}\left(U, \mathcal{O}_{K^{p}}^{\text {la }}\right)=0
$$

for any $U \in \mathfrak{B}$ and $j>0$. Therefore, $H^{i}\left(\mathscr{F} \ell, \mathcal{O}_{K^{p}}^{\text {la }}\right)$ can also be computed using Čech cohomology. Recall that $\mathfrak{B}$ is stable under finite intersections. By Corollaire 4, p. 176 of [Gro57], it suffices to show the Čech cohomology $\check{H}^{j}\left(U, \mathcal{O}_{K^{p}}^{\text {la }}\right)=0$ for any $U \in \mathfrak{B}$ and $j>0$. This can be proved in exactly 
the same way as in the first paragraph of the proof of Corollary 3.6.10 using the acyclicity result in Proposition 4.3.15.

Now let $\mathfrak{U} \subset \mathfrak{B}$ be a finite cover of $\mathscr{F} \ell$ and $C^{\bullet}\left(\mathfrak{H}, \mathcal{O}_{K^{p}}\right), C^{\bullet}\left(\mathfrak{H}, \mathcal{O}_{K^{p}}^{\text {la }}\right)$ be the Čech complexes for $\mathcal{O}_{K^{p}}, \mathcal{O}_{K^{p}}^{\text {la }}$ using this cover. Then $C^{\bullet}\left(\mathfrak{H}, \mathcal{O}_{K^{p}}\right)$ is a strict complex because $H^{i}\left(C^{\bullet}\left(\mathfrak{H}, \mathcal{O}_{K^{p}}^{+}\right)\right) \cong$ $H^{i}\left(\mathcal{X}_{K^{p}}, \mathcal{O}_{\mathcal{X}_{K} p}^{+}\right) \cong \tilde{H}^{i}\left(K^{p}, \mathcal{O}_{C}\right)$ (as almost $\mathcal{O}_{C}$-modules) has bounded $p$-power torsion, where $\mathcal{O}_{K^{p}}^{+}=$ $\pi_{\mathrm{HT} *} \mathcal{O}_{\mathcal{X}_{K} p}^{+}$. Moreover, each $C^{i}\left(\mathfrak{H}, \mathcal{O}_{K^{p}}\right)$ is $\mathfrak{Q} \mathfrak{A}$-acyclic by Proposition 4.3 .15 and $H^{i}\left(C^{\bullet}\left(\mathfrak{H}, \mathcal{O}_{K^{p}}\right)\right)=$ $\tilde{H}^{i}\left(K^{p}, C\right)$ is $\mathfrak{Q} \mathfrak{A}$-acyclic because $\tilde{H}^{i}\left(K^{p}, \mathbb{Z}_{p}\right)$ is an admissible representation of $\mathrm{GL}_{2}\left(\mathbb{Q}_{p}\right)$ and we can apply the result of Schneider-Teitelbaum; cf. Corollary 2.2.4. Hence, the theorem follows from the second part of Lemma 2.2.2 as $\left(C^{\bullet}\left(\mathfrak{U}, \mathcal{O}_{K^{p}}\right)\right)^{\text {la }}=C^{\bullet}\left(\mathfrak{H}, \mathcal{O}_{K^{p}}^{\text {la }}\right)$.

\section{5. $\mu$-isotypic part of completed cohomology}

The goal of this section is to determine the $\mu$-isotypic part of $\tilde{H}^{i}\left(K^{p}, C\right)^{\text {la }}$. We will give a complete answer for integral weights as described in the Introduction. Roughly speaking, the answer is a mixture of coherent cohomology groups of modular curves at finite level and overconvergent modular forms. Also, we will give a $p$-adic Hodge-theoretic interpretation of the horizontal action $\theta_{\mathfrak{h}}$.

From now on, we assume $C=\mathbb{C}_{p}$ is the completion of $\overline{\mathbb{Q}}_{p}$. Then $G_{\mathbb{Q}_{p}}$ acts continuously on $C, \tilde{H}^{i}\left(K^{p}, \mathbb{Z}_{p}\right), \tilde{H}^{i}\left(K^{p}, C\right)$ and commutes with the action of $\mathrm{GL}_{2}\left(\mathbb{Q}_{p}\right)$ and Hecke operators away from $p$.

\subsection{A p-adic Hodge-theoretic interpretation of $\theta_{\mathfrak{h}}$}

5.1.1. We would like to write down the action $\theta_{\mathfrak{h}}$ introduced in Corollary 4.2 .8 on $\mathcal{O}_{K^{p}}^{\text {la }}$ using the explicit description in Theorem 4.3.9. So keep the notation in Subsection 4.3. In particular, $e_{1}$ generates $H^{0}\left(V, \omega_{K^{p}}\right)$. Then for any $f \in \mathcal{O}_{K^{p}}^{\text {la }}(U)$, we can write

$$
f=\sum_{i, j, k \geq 0} c_{i, j, k}^{(n)}(f)\left(x-x_{n}\right)^{i}\left(\log \left(\frac{e_{1}}{e_{1, n}}\right)\right)^{j}\left(\log \left(\frac{t}{t_{n}}\right)\right)^{k}
$$

for sufficiently large $n$ (as in Theorem 4.3.9). By our construction in Corollary 4.2.8, a direct computation shows that $\theta_{\mathfrak{h}}\left(\left(\begin{array}{ll}a & 0 \\ 0 & d\end{array}\right)\right)$ acts on $\mathcal{O}_{K^{p}}^{\text {la }}(U)$ as $\left(\begin{array}{cc}d & (d-a) x \\ 0 & a\end{array}\right) \in \mathcal{O}_{\mathscr{F} \ell}(U) \otimes_{C} \mathfrak{g}$. (To see this, recall that $\left(\begin{array}{cc}x & x^{2} \\ -1 & -x\end{array}\right)$ is a generator of $H^{0}\left(U, \mathfrak{n}^{0}\right)$; cf. Section 4.3.2. One computes directly that $\left[\left(\begin{array}{cc}d & (d-a) x \\ 0 & a\end{array}\right),\left(\begin{array}{cc}x & x^{2} \\ -1 & -x\end{array}\right)\right]=$ $(a-d)\left(\begin{array}{cc}x & x^{2} \\ -1 & -x\end{array}\right)$, and this $(a-d)$ agrees with $\left.\left[\left(\begin{array}{ll}a & 0 \\ 0 & d\end{array}\right),\left(\begin{array}{ll}0 & 1 \\ 0 & 0\end{array}\right)\right]=(a-d)\left(\begin{array}{ll}0 & 1 \\ 0 & 0\end{array}\right).\right)$ Hence,

$$
c_{i, j, k}^{(n)}\left(\theta_{\mathfrak{h}}\left(\left(\begin{array}{ll}
a & 0 \\
0 & d
\end{array}\right)\right) \cdot f\right)=d(j+1) c_{i, j+1, k}^{(n)}(f)+(a+d)(k+1) c_{i, j, k+1}^{(n)}(f) .
$$

Let $\chi$ be a weight of $\mathfrak{h}$; that is, a $C$-linear map $\chi: \mathfrak{h} \rightarrow C$. We can write $\chi\left(\left(\begin{array}{ll}a & 0 \\ 0 & d\end{array}\right)\right)=a n_{1}+d n_{2}$ for some $n_{1}, n_{2} \in C$. Fix $N$ sufficiently large so that

$$
\begin{aligned}
\left(\frac{t}{t_{N}}\right)^{n_{1}} & :=\sum_{l \geq 0}\left(\begin{array}{c}
n_{1} \\
l
\end{array}\right)\left(\frac{t}{t_{N}}-1\right)^{l}, \\
\left(\frac{e_{1}}{e_{1, N}}\right)^{n_{2}-n_{1}} & :=\sum_{l \geq 0}\left(\begin{array}{c}
n_{2}-n_{1} \\
l
\end{array}\right)\left(\frac{e_{1}}{e_{1, N}}-1\right)^{l}
\end{aligned}
$$


converge in $\mathcal{O}_{K^{p}}^{G_{r(N)}-\text { an }}$. One checks easily that $\theta_{\mathfrak{h}}\left(\left(\begin{array}{ll}a & 0 \\ 0 & d\end{array}\right)\right)$ acts as $\chi$ on $\left(\frac{t}{t_{N}}\right)^{n_{1}}\left(\frac{e_{1}}{e_{1, N}}\right)^{n_{2}-n_{1}}$. Denote by $\mathcal{O}_{K^{p}}^{\text {la, } \chi} \subset \mathcal{O}_{K^{p}}^{\text {la }}$ the subsheaf of sections of weight $\chi$.

Lemma 5.1.2. For any weight $\chi$ and $U \in \mathfrak{B}$,

(1) $H^{i}\left(\mathfrak{h}, \mathcal{O}_{K^{p}}^{\mathrm{la}}(U) \otimes \chi\right)=0, i \geq 1$.

(2) Suppose $e_{1}$ is a generator on $V=\pi_{\mathrm{HT}}^{-1}(U)$; then any $f \in \mathcal{O}_{K^{p}}^{\mathrm{la}, \chi}(U)$ can be written as

$$
f=\left(\frac{t}{t_{N}}\right)^{n_{1}}\left(\frac{e_{1}}{e_{1, N}}\right)^{n_{2}-n_{1}} \sum_{i \geq 0} c_{i}^{(n)}(f)\left(x-x_{n}\right)^{i}
$$

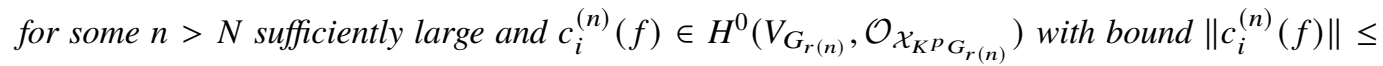
$C^{\prime} p^{(n-1) i}$ for a uniform $C^{\prime}$.

Proof. Using the action of $\mathrm{GL}_{2}\left(\mathbb{Q}_{p}\right)$, we can reduce to the case considered above; that is, that $e_{1}$ generates $H^{0}\left(V, \omega_{K^{p}}\right)$. Note that $\left(\frac{t}{t_{N}}\right)^{n_{1}}\left(\frac{e_{1}}{e_{1, N}}\right)^{n_{2}-n_{1}}$ is invertible. Hence, multiplication by it induces an $\mathfrak{h}$-equivariant isomorphism $\mathcal{O}_{K^{p}}^{\text {la }}(U) \otimes \chi \stackrel{\sim}{\rightarrow} \mathcal{O}_{K^{p}}^{\text {la }}(U)$. Therefore, it is enough to prove the case $\chi=0$. The second part is clear in view of the explicit formula for $c_{i, j, k}^{(n)}\left(\theta_{\mathfrak{h}}\left(\left(\begin{array}{ll}a & 0 \\ 0 & d\end{array}\right)\right) \cdot f\right)$ above. To see the first part, write $\mathfrak{a}=\left\{\left(\begin{array}{ll}* & 0 \\ 0 & 0\end{array}\right)\right\} \subset \mathfrak{h}$. We claim

(1) $H^{1}\left(\mathfrak{a}, \mathcal{O}_{K^{p}}^{\mathrm{la}}(U)\right)=0$;

(2) $H^{0}\left(\mathfrak{a}, \mathcal{O}_{K^{p}}^{\text {la }}(U)\right) \subset \mathcal{O}_{K^{p}}^{\text {la }}(U)$ is the subset of $f$ such that $c_{i, j, k}^{(n)}(f)=0, k \geq 1$.

Again, the second claim is clear by our explicit formula. For the first claim, suppose $f \in \mathcal{O}_{K^{p}}^{\text {la }}(U)$ has an expansion as in Theorem 4.3.9. For any $i, j, k \geq 0$, let $c_{i, j, k+1}^{\prime}=\frac{1}{k+1} c_{i, j, k}^{(n)}(f)$. Then $\left\|c_{i, j, k}^{\prime}\right\| \leq$ $C^{\prime \prime} p^{(n-0.5)(i+j+k)}$ for some uniform $C^{\prime \prime}$. Hence,

$$
f^{\prime}=\sum_{i, j \geq 0, k \geq 1} c_{i, j, k}^{\prime}\left(x-x_{n}\right)^{i}\left(\log \left(\frac{e_{1}}{e_{1, n}}\right)\right)^{j}\left(\log \left(\frac{t}{t_{n}}\right)\right)^{k}
$$

converges in $\mathcal{O}_{K^{p}}^{G_{r(n)}-\text { an }}$. One checks easily $\theta_{\mathfrak{h}}\left(\left(\begin{array}{ll}1 & 0 \\ 0 & 0\end{array}\right)\right) \cdot f^{\prime}=f$. This proves the vanishing of $H^{1}\left(\mathfrak{a}, \mathcal{O}_{K^{p}}^{\text {la }}(U)\right)$. The same argument also gives $H^{1}\left(\mathfrak{h} / \mathfrak{a}, H^{0}\left(\mathfrak{a}, \mathcal{O}_{K^{p}}^{\text {la }}(U)\right)\right)=0$. By the HochschildSerre spectral sequence, we deduce our claim in the lemma.

We denote by $H^{i}\left(\mathscr{F} \ell, \mathcal{O}_{K^{p}}^{\text {la }}\right)^{\chi}$ the subspace of $H^{i}\left(\mathscr{F} \ell, \mathcal{O}_{K^{p}}^{\text {la }}\right)$ where $\theta_{\mathfrak{h}}$ acts by $\chi$. Then $H^{0}\left(\mathscr{F} \ell, \mathcal{O}_{K^{p}}^{\mathrm{la}, \chi}\right)=H^{0}\left(\mathscr{F} \ell, \mathcal{O}_{K^{p}}^{\mathrm{la}}\right)^{\chi}$.

\section{Corollary 5.1.3.}

(1) $\theta_{\mathfrak{h}}(h) \cdot f=0, \theta_{\mathfrak{h}}(z) \cdot f=z \cdot f, f \in H^{0}\left(\mathscr{F} \ell, \mathcal{O}_{K^{p}}^{\text {la }}\right)$. In particular, $H^{0}\left(\mathscr{F} \ell, \mathcal{O}_{K^{p}}^{\text {la, } \chi}\right)=0$ if $\chi(h) \neq 0$.

(2) If $\chi(h) \neq 0$, then $H^{1}\left(\mathscr{F} \ell, \mathcal{O}_{K^{p}}^{\text {la }, \chi}\right)=H^{1}\left(\mathscr{F} \ell, \mathcal{O}_{K^{p}}^{\text {la }}\right)^{\chi}$. If $\chi(h)=0$, there is a $\mathfrak{g}$-equivariant exact sequence

$$
0 \rightarrow \underset{K_{p} \subset \underset{\mathrm{GL}_{2}}{\overrightarrow{\lim }}\left(\mathbb{Q}_{p}\right)}{ } H^{0}\left(\mathcal{X}_{K^{p} K_{p}}, \mathcal{O}_{\mathcal{X}_{K}{ }^{K_{p}}}\right) \cdot\left(\frac{t}{t_{N}}\right)^{n_{1}} \rightarrow H^{1}\left(\mathscr{F} \ell, \mathcal{O}_{K^{p}}^{\mathrm{la}, \chi}\right) \rightarrow H^{1}\left(\mathscr{F} \ell, \mathcal{O}_{K^{p}}^{\mathrm{la}}\right)^{\chi} \rightarrow 0,
$$

where $n_{1}=\chi\left(\left(\begin{array}{ll}1 & 0 \\ 0 & 0\end{array}\right)\right)$ and $t_{N} \in H^{0}\left(\mathcal{X}_{K^{p} K_{p}}, \mathcal{O}_{\mathcal{X}_{K^{p} K_{p}}}\right)$ sufficiently close to t for some $K_{p}$. 
Proof. The action of $\mathrm{GL}_{2}\left(\mathbb{Q}_{p}\right)$ on $\tilde{H}^{0}\left(K^{p}, C\right)^{\text {la }}$ factors through the determinant map; hence, for any global section $f \in H^{0}\left(\mathscr{F} \ell, \mathcal{O}_{K}^{\text {la }}\right) \cong \tilde{H}^{0}\left(K^{p}, C\right)^{\text {la }}$, we have $\theta_{\mathfrak{h}}(h) \cdot f=0$ and $\theta_{\mathfrak{h}}(z) \cdot f=z \cdot f$ by the explicit expression of $\theta_{\mathfrak{h}}$ in Section 5.1.1. This also shows that the horizontal action $\theta_{\mathfrak{h}}$ of $\mathfrak{h}$ agrees with the constant action of $\mathfrak{h} \subset \mathfrak{g}$ on $\tilde{H}^{0}\left(K^{p}, C\right)^{\mathrm{la}}$.

For the second part, it follows from the first part of Lemma 5.1.2 that there is a spectral sequence

$$
E_{2}^{p q}=\operatorname{Ext}_{C[\mathfrak{b}]}^{p}\left(\chi, H^{q}\left(\mathscr{F} \ell, \mathcal{O}_{K^{p}}^{\mathrm{la}}\right)\right) \Rightarrow H^{p+q}\left(\mathscr{F} \ell, \mathcal{O}_{K^{p}}^{\mathrm{la}, \chi}\right)
$$

The exact sequence of low degrees reads ${ }^{2}$

$$
0 \rightarrow \operatorname{Ext}_{C[\mathfrak{b}]}^{1}\left(\chi, H^{0}\left(\mathscr{F} \ell, \mathcal{O}_{K^{p}}^{\mathrm{la}}\right)\right) \rightarrow H^{1}\left(\mathscr{F} \ell, \mathcal{O}_{K^{p}}^{\mathrm{la}, \chi}\right) \rightarrow H^{1}\left(\mathscr{F} \ell, \mathcal{O}_{K^{p}}^{\mathrm{la}}\right)^{\chi} \rightarrow \operatorname{Ext}_{C[\mathfrak{b}]}^{2}\left(\chi, H^{0}\left(\mathscr{F} \ell, \mathcal{O}_{K^{p}}^{\mathrm{la}}\right)\right)
$$

When $\chi(h) \neq 0$, both $\operatorname{Ext}^{1}$ and $\operatorname{Ext}^{2}$ vanish because $\theta_{\mathfrak{h}}(h)$ acts via zero on $H^{0}\left(\mathscr{F} \ell, \mathcal{O}_{K^{p}}^{\text {la }}\right)$. Now assume $\chi(h)=0$. After multiplying by $\left(\frac{t}{t_{N}}\right)^{-n_{1}} \in H^{0}\left(\mathscr{F} \ell, \mathcal{O}_{K}^{\text {la }}\right)^{-\chi}$, we may assume $\chi=0$. It suffices to show

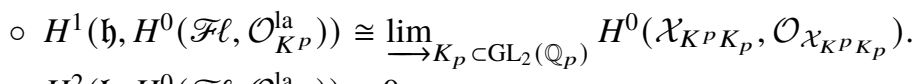

○ $H^{2}\left(\mathfrak{h}, H^{0}\left(\mathscr{F} \ell, \mathcal{O}_{K}^{\mathrm{la}}\right)\right)=0$.

Both claims follow from the Hochschild-Serre spectral sequence and

○ $H^{1}\left(\mathfrak{a}, \tilde{H}^{0}\left(K^{p}, C\right)^{\mathrm{la}}\right)=0$;

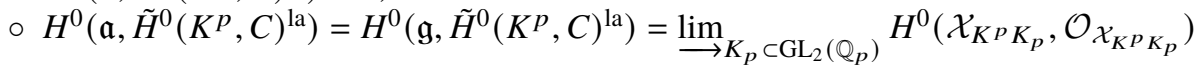

by the explicit description of $\tilde{H}^{0}\left(K^{p}, C\right)$ in [Eme06b, (4.2)].

5.1.4. It is interesting to investigate the $p$-adic Hodge-theoretic meaning of $\theta_{\mathfrak{h}}$. First, we generalise the classical notion of the Sen operator.

Definition 5.1.5. Suppose $W$ is a $C$-Banach space equipped with a semi-linear continuous action of an open subgroup of $G_{\mathbb{Q}_{p}}$, say $G_{K}$. We say a continuous $C$-linear endomorphism $\theta_{\operatorname{Sen}} \in \operatorname{End}_{C}(W)$ is a $\operatorname{Sen}$

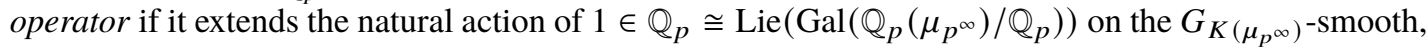
$G_{K}$-locally analytic vectors of $W$ (viewed as a $\mathbb{Q}_{p}$-Banach space).

If $W=\lim _{\longrightarrow} W_{n}$ is an increasing union of $C$-Banach spaces $W_{n}$ equipped with a semi-linear continuous action of an open subgroup of $G_{\mathbb{Q}_{p}}$, then we say $\theta \in \operatorname{End}_{C}(W)$ is a Sen operator if $\theta$ preserves each $W_{n}$ and acts as a Sen operator on it. We also say $W$ has pure Hodge-Tate-Sen weight $k \in C$ if multiplication by $-k$ is a Sen operator on $W$.

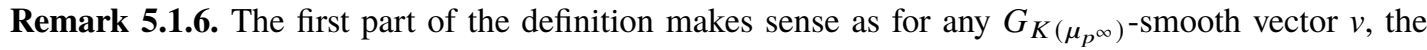
action of $G_{K}$ on $v$ factors through a finite-dimensional $p$-adic Lie group which has an open subgroup naturally isomorphic to an open subgroup of $\operatorname{Gal}\left(\mathbb{Q}_{p}\left(\mu_{p^{\infty}}\right) / \mathbb{Q}_{p}\right)$. Also, it is clear that this definition is independent of the choice of $K$.

Remark 5.1.7. If $W$ is a finite-dimensional $C$-vector space, then in [Sen81] Sen proves that $\theta_{\text {Sen }}$ exists and is unique. However, for a general $W$, to what extent $\theta_{\text {Sen }}$ exists uniquely is not known to the author.

In our case, we will take $W=\mathcal{O}_{K^{p}}(U)^{\mathrm{la}}$ and $\tilde{H}^{i}\left(K^{p}, C\right)^{\mathrm{la}}$. Note that $V_{K_{p}}$ can be defined over a finite extension $K$ of $\mathbb{Q}_{p}$ so $\mathcal{O}_{K^{p}}(U)^{\text {la }}$ has a natural action of $G_{K}$.

Theorem 5.1.8. $\theta_{\mathfrak{h}}\left(\begin{array}{ll}0 & 0 \\ 0 & 1\end{array}\right)$ is the unique Sen operator on $\mathcal{O}_{K^{p}}(U)^{\mathrm{la}}$.

\footnotetext{
${ }^{2}$ In fact, one can avoid the machinery of spectral sequences in this simple case. It was shown in the proof of Theorem 4.4.6 that $H^{i}\left(\mathscr{F} \ell, \mathcal{O}_{K}^{\text {la }}\right)$ can be computed by the Čech cohomology. Hence, we can use the cover $\left\{U_{1}, U_{2}\right\}$ of $\mathscr{F} \ell$ introduced in Section 5.1.12. Therefore, $\mathcal{O}_{K}^{\text {la }} p\left(U_{1}\right) \oplus \mathcal{O}_{K}^{\text {la }}\left(U_{2}\right) \rightarrow \mathcal{O}_{K}^{\text {la }} p\left(U_{1} \cap U_{2}\right)$ computes $H^{i}\left(\mathscr{F} \ell, \mathcal{O}_{K}^{\text {la }}\right)$. Now this exact sequence comes from applying $\operatorname{Ext}_{C[\mathfrak{b}]}^{\bullet}(\chi, \cdot)$ to this Čech complex.
} 
Remark 5.1.9. Roughly speaking, this result relates an operator in $p$-adic Hodge theory on (the infinite level) modular curves with some group-theoretic operator $\left(\theta_{\mathfrak{h}}\right)$. This is very classical in the study of the cohomology of locally symmetric spaces using complex Hodge theory. See, for example, Chapter II, Section 4 of [BW00].

Remark 5.1.10. This result and Theorem 5.1 .11 are all obtained by explicit calculations. It should be possible to avoid these calculations by further decompleting $\mathcal{O}_{K^{p}}(U)^{\text {la }}$ with respect to the action of $G_{K}$ (i.e., usual Sen theory). I plan to come back to this in a future work.

Proof. By Theorem 4.3.9, for each $m$, we have continuous embeddings

$$
\mathcal{O}_{K^{p}}(U)^{G_{m}-\mathrm{an}} \rightarrow \mathcal{O}^{n}(U)\left\{x, e_{1}, t\right\} \rightarrow \mathcal{O}_{K^{p}}(U)^{G_{r(n)} \text { an }}
$$

for some $n$. See Definition 4.3.10. Since elements defined over a finite extension of $K$ are dense in $H^{0}\left(V_{G_{r(n)}}, \mathcal{O}_{\mathcal{X}_{K} p_{G_{r(n)}}}\right)$, we may assume $x_{n}, e_{1, n}, t_{n}$ defined over $K$ after enlarging $K$ if necessary. Hence, $G_{K}$ preserves $\mathcal{O}^{n}(U)\left\{x, e_{1}, t\right\}$ and it is enough to show that the action of $\left.\theta_{\mathfrak{h}}\left(\begin{array}{ll}0 & 0 \\ 0 & 1\end{array}\right)\right)$ is a Sen operator on $\mathcal{O}^{n}(U)\left\{x, e_{1}, t\right\}$ and is the unique one.

For simplicity, we write $M$ for $\mathcal{O}^{n}(U)\left\{x, e_{1}, t\right\}$, and for any finite extension $K^{\prime}$ of $K$, we denote by $M_{K^{\prime}} \subset M$ the subspace of $f$ with all $c_{i, j, k}^{(n)}(f)$ defined over $K^{\prime}$. It is clear that

$$
M \cong M_{K^{\prime}} \widehat{\otimes}_{K^{\prime}} C .
$$

One useful fact is

○ $G_{\mathbb{Q}_{p}}$ acts trivially on $x$ and acts via cyclotomic character on $e_{1}, t$.

From this, one can check that $M_{K^{\prime}}$ is $G_{K^{\prime}\left(\mu_{p^{\infty}}\right)}$-fixed and $G_{K^{\prime}}$ acts analytically on it. Conversely, any such element $f$ of $M$ is contained in $M_{K^{\prime}}$ because $c_{i, j, k}^{(n)}(f)$ can be computed from $f$ using the action of $\mathfrak{g}$ as in Subsection 4.3; hence, is $G_{K^{\prime}}$-analytic and an argument using Tate's normalised trace implies that it is in fact fixed by $G_{K^{\prime}}$. Now a direct computation (using results in Section 5.1.1) shows that $\theta_{\mathfrak{h}}\left(\left(\begin{array}{ll}0 & 0 \\ 0 & 1\end{array}\right)\right)$ agrees with the natural action of $1 \in \mathbb{Q}_{p} \cong \operatorname{Lie}\left(\operatorname{Gal}\left(\mathbb{Q}_{p}\left(\mu_{p^{\infty}}\right) / \mathbb{Q}_{p}\right)\right)$ on $M_{K^{\prime}}$. The uniqueness follows from $M \cong M_{K^{\prime}} \widehat{\otimes}_{K^{\prime}} C$.

Theorem 5.1.11. $\left.\theta_{\mathfrak{h}}\left(\begin{array}{ll}0 & 0 \\ 0 & 1\end{array}\right)\right)$ is the unique Sen operator on $H^{i}\left(\mathscr{F} \ell, \mathcal{O}_{K^{p}}^{\mathrm{la}}\right)=\tilde{H}^{i}\left(K^{p}, C\right)^{\mathrm{la}}$ for any $i$.

5.1.12. The case $i=0$ follows from Theorem 5.1.8. So it suffices to prove the case $i=1$. We introduce some notation first.

We have shown in the proof of Theorem 4.4.6 that $H^{i}\left(\mathscr{F} \ell, \mathcal{O}_{K^{p}}^{\text {la }}\right)$ can be computed by Čech cohomology using a finite cover of $\mathscr{F} \ell$ in $\mathfrak{B}$. In particular, we can use the cover $\left\{U_{1}, U_{2}\right\}$, where $U_{1}$ (respectively $U_{2}$ ) is the subset $|x| \leq 1$ (respectively $|x| \geq 1$ ). See Theorem 4.1.7. Denote by $U_{12}=U_{1} \cap U_{2}$. Then

$$
\mathcal{O}_{K^{p}}^{\mathrm{la}}\left(U_{1}\right) \oplus \mathcal{O}_{K^{p}}^{\mathrm{la}}\left(U_{2}\right) \rightarrow \mathcal{O}_{K^{p}}^{\mathrm{la}}\left(U_{12}\right)
$$

computes $H^{i}\left(\mathscr{F} \ell, \mathcal{O}_{K^{p}}^{\text {la }}\right)$. Denote by $V_{\text {? }}=\pi_{\mathrm{HT}}^{-1}\left(U_{?}\right)$ with $?=1,2,12$. Fix an open subgroup $G_{0}=$ $1+p^{l} M_{2}\left(\mathbb{Z}_{p}\right)$ for some sufficiently large $l \geq 2$ so that $V_{\text {? }}$ is the preimage of some affinoid subset $V_{?, G_{0}}$ of $\mathcal{X}_{K^{p} G_{0}}$. As before, we write $G_{n}=G_{0}^{p^{n}}$. Since $e_{1}$ is a basis on $U_{1}$, we can find $x_{n}, e_{1, n}, t_{n}$ as in Section 4.3.5 and define $\mathcal{O}^{n}\left(U_{1}\right)\left\{x, e_{1}, t\right\}$ as in Definition 4.3.10. Note that by restricting $x_{n}, e_{1, n}, t_{n}$ on $U_{12}$, we can define $\mathcal{O}^{n}\left(U_{12}\right)\left\{x, e_{1}, t\right\}$ similarly with unit ball $\mathcal{O}^{n}\left(U_{12}\right)^{+}\left\{x, e_{1}, t\right\}$. Now $e_{1}$ is not a basis on $U_{2}$. So we work with $\frac{1}{x}, e_{2}, t$ instead of $x, e_{1}, t$. Write $y=\frac{1}{x}=\frac{e_{1}}{e_{2}}$. Since the action of $w=\left(\begin{array}{ll}0 & 1 \\ 1 & 0\end{array}\right)$ 
interchanges $U_{1}, U_{2}$, we obtain $y_{n}:=w^{*} x_{n}, e_{2, n}:=w^{*} e_{1, n}$ on $U_{2}$. Using $y_{n}, e_{2, n}, t_{n}$, we can define $\mathcal{O}^{n}\left(U_{2}\right)\left\{y, e_{2}, t\right\} \subset \mathcal{O}_{K^{p}}^{\text {la }}\left(U_{2}\right)$ on $U_{2}$.

Lemma 5.1.13. For any $n>2$, the restriction from $U_{2}$ to $U_{12}$ induces a map

$$
\mathcal{O}^{n}\left(U_{2}\right)\left\{y, e_{2}, t\right\} \rightarrow \mathcal{O}^{n}\left(U_{12}\right)\left\{x, e_{1}, t\right\}
$$

preserving norms of $y-y_{n}$ and $\log \left(\frac{e_{2}}{e_{2, n}}\right)$. See Definition 4.3.10 for the definition of norm $\|\cdot\|_{n}$.

Proof. On $U_{12}$, we have

$$
y-y_{n}=\frac{1}{x}-y_{n}=\frac{1}{x_{n}} \cdot \frac{1}{1+\frac{x-x_{n}}{x_{n}}}-y_{n}=\left(\frac{1}{x_{n}}-y_{n}\right)-\sum_{i \geq 1} \frac{1}{\left(-x_{n}\right)^{i+1}}\left(x-x_{n}\right)^{i} .
$$

Since $\left\|x_{n}\right\|=\|x\|=1$ and $\frac{1}{x_{n}}-y_{n}=\left(\frac{1}{x_{n}}-\frac{1}{x}\right)+\left(y-y_{n}\right)$ has norm $\leq p^{-n}$ on $U_{12}$; hence,

$$
p^{-(n-1)}\left(y-y_{n}\right) \in-\frac{1}{x_{n}^{2}} p^{-(n-1)}\left(x-x_{n}\right)+p \mathcal{O}^{n}\left(U_{12}\right)^{+}\left\{x, e_{1}, t\right\} .
$$

The claim for $\log \left(\frac{e_{2}}{e_{2, n}}\right)$ can be proved in a similar way.

Proof of Theorem 5.1.11. Fix an integer $m>2$. Recall that $\tilde{H}^{1}\left(K^{p}, C\right)=\operatorname{coker}\left(\mathcal{O}_{K^{p}}\left(U_{1}\right) \oplus\right.$ $\left.\mathcal{O}_{K^{p}}\left(U_{2}\right) \rightarrow \mathcal{O}_{K^{p}}\left(U_{12}\right)\right)$. By Proposition 4.3.15 and Corollary 2.2.4, all of the terms in the following exact sequence

$$
0 \rightarrow \tilde{H}^{0}\left(K^{p}, C\right) \rightarrow \mathcal{O}_{K^{p}}\left(U_{1}\right) \oplus \mathcal{O}_{K^{p}}\left(U_{2}\right) \rightarrow \mathcal{O}_{K^{p}}\left(U_{12}\right)
$$

are strongly $\mathfrak{Q} \mathfrak{A}$-acyclic with respect to the action of $G_{0}$. From this, it is easy to see that there exists an integer $m^{\prime} \geq m$ such that $\tilde{H}^{1}\left(K^{p}, C\right)^{G_{m} \text {-an }}$ is contained in the image of $\mathcal{O}_{K^{p}}\left(U_{12}\right)^{G_{m^{\prime}} \text {-an }}$. Then by Theorem 4.3.9, we can find $n \geq m^{\prime}$ so that $\mathcal{O}_{K^{p}}\left(U_{12}\right)^{G_{m^{\prime}} \text {-an }} \subset \mathcal{O}^{n}\left(U_{12}\right)\left\{x, e_{1}, t\right\} \subset \mathcal{O}_{K^{p}}\left(U_{12}\right)^{G_{r(n)} \text {-an }}$. As a consequence, the inclusion $\tilde{H}^{1}\left(K^{p}, C\right)^{G_{m} \text {-an }} \subset \tilde{H}^{1}\left(K^{p}, C\right)^{G_{r(n)} \text {-an }}$ factors through the largest separated quotient $M^{n}$ of

$$
\operatorname{coker}\left(\mathcal{O}^{n}\left(U_{1}\right)\left\{x, e_{1}, t\right\} \oplus \mathcal{O}^{n}\left(U_{2}\right)\left\{y, e_{2}, t\right\} \rightarrow \mathcal{O}^{n}\left(U_{12}\right)\left\{x, e_{1}, t\right\}\right)
$$

(using the quotient topology on the cokernel). It suffices to show that $\left.\theta_{\mathfrak{h}}\left(\begin{array}{ll}0 & 0 \\ 0 & 1\end{array}\right)\right)$ acts as the unique Sen operator on $M^{n}$.

Now let $K$ be a finite extension of $\mathbb{Q}_{p}$ so that $V_{1, G_{0}}, x_{n}, e_{1, n}, t_{n}$ are all defined over $K$. Denote by $M_{1} \subset \mathcal{O}^{n}\left(U_{1}\right)\left\{x, e_{1}, t\right\}$ the subspace of $G_{K\left(\mu_{p^{\infty}}\right)}$-fixed, $G_{K}$-analytic vectors. This is a $K$-Banach algebra with norm $\|\cdot\|_{n}$, and we denote its unit ball by $M_{1}^{o}$. Similarly, we can define $M_{2} \subset \mathcal{O}^{n}\left(U_{2}\right)\left\{y, e_{2}, t\right\}$ and $M_{12} \subset \mathcal{O}^{n}\left(U_{12}\right)\left\{x, e_{1}, t\right\}$ and their unit balls $M_{2}^{o}, M_{12}^{o}$. Then as in the proof of Theorem 5.1.8, we have $M_{1}^{o} \widehat{\otimes}_{\mathcal{O}_{K}} C \cong \mathcal{O}^{n}\left(U_{1}\right)\left\{x, e_{1}, t\right\}$, and similar results hold for $M_{12}$ and $M_{2}$. The previous lemma implies that $\mathcal{O}_{K^{p}}^{\text {la }}\left(U_{1}\right) \oplus \mathcal{O}_{K^{p}}^{\text {la }}\left(U_{2}\right) \rightarrow \mathcal{O}_{K^{p}}^{\text {la }}\left(U_{12}\right)$ has a subcomplex $M_{1}^{o} \oplus M_{2}^{o} \rightarrow M_{12}^{o}$. Moreover, let $M^{o}$ be the quotient of $\operatorname{coker}\left(M_{1}^{o} \oplus M_{2}^{o} \rightarrow M_{12}^{o}\right)$ by its torsion subgroup. Then

$$
M^{n}=M^{o} \widehat{\otimes}_{\mathcal{O}_{K}} C
$$

and $M=M^{o} \otimes_{\mathbb{Z}_{p}} \mathbb{Q}_{p}$ is the subspace of $G_{K\left(\mu_{p^{\infty}}\right)}$-fixed $G_{K}$-analytic vectors in $M^{n}$. As in the proof of Theorem 5.1.8, we know that $\theta_{\mathfrak{h}}\left(\left(\begin{array}{ll}0 & 0 \\ 0 & 1\end{array}\right)\right)$ agrees with $1 \in \operatorname{Lie}\left(\operatorname{Gal}\left(\mathbb{Q}_{p}\left(\mu_{p^{\infty}}\right) / \mathbb{Q}_{p}\right)\right)$ on $M_{12}$ and hence also agrees on its quotient $M$. This verifies our definition of Sen operator. 
Remark 5.1.14. It can be proved by explicit calculations that $\operatorname{coker}\left(M_{1}^{o} \oplus M_{2}^{o} \rightarrow M_{12}^{o}\right)$ is already torsion-free, so taking the largest separated quotient is in fact unnecessary.

Remark 5.1.15. It is clear from the proof that there is a close relation between two analytic aspects of the completed cohomology: one comes from the group action of $\mathrm{GL}_{2}\left(\mathbb{Q}_{p}\right)$, and one comes from the Galois action of $G_{\mathbb{Q}_{p}}$. Also, Theorem 5.1.11 implies that the two infinitesimal characters are closely related. Both are actually deep theorems in the $p$-adic local Langlands for $\mathrm{GL}_{2}\left(\mathbb{Q}_{p}\right)$. See Théorème V.3 of [CD14] and Théorème 1.2 of [Dos12].

Remark 5.1.16. It is natural to ask whether we can show the existence of the Sen operator on $\tilde{H}^{i}\left(K^{p}, C\right)^{\text {la }}$ in a more direct way. The answer is affirmative. We sketch a construction here based on the Tate-Sen formalism of Berger-Colmez [BC08]. Let $G=G_{n}$ for some $n$. The key point here is that

$\circ$ the action of $G_{\mathbb{Q}_{p}}$ on $\tilde{H}^{i}\left(K^{p}, \mathbb{Q}_{p}\right)^{G-a n, o} / p$ is trivial on an open subgroup; that is, the image of $G_{\mathbb{Q}_{p}} \rightarrow$ End $\left(\left(\tilde{H}^{i}\left(K^{p}, \mathbb{Q}_{p}\right)^{G-a n}\right)^{o} / p\right)$ is finite, where $\tilde{H}^{i}\left(K^{p}, \mathbb{Q}_{p}\right)^{G-a n, o}$ denotes the unit open ball of $\tilde{H}^{i}\left(K^{p}, \mathbb{Q}_{p}\right)^{G-\text { an }}$.

The argument is almost the same as the proof of [Pan20, Theorem 6.1]. Let $W^{o}$ be the unit open ball of $\tilde{H}^{i}\left(K^{p}, \mathbb{Q}_{p}\right)$. Then

$$
\begin{aligned}
\tilde{H}^{i}\left(K^{p}, \mathbb{Q}_{p}\right)^{G-\mathrm{an}, o} / p & =\left(W^{o} \widehat{\otimes}_{\mathbb{Z}_{p}} \mathscr{C}^{\mathrm{an}}\left(G_{n}, \mathbb{Q}_{p}\right)^{o}\right)^{G_{n}} / p \\
& \subseteq\left(W^{o} \otimes_{\mathbb{Z}_{p}} \mathscr{C}^{\mathrm{an}}\left(G_{n}, \mathbb{Q}_{p}\right)^{o} / p\right)^{G_{n}} \\
& \subseteq\left(W^{o} / p W^{o} \otimes_{\mathbb{F}_{p}} \mathscr{C}^{\mathrm{an}}\left(G_{n}, \mathbb{Q}_{p}\right)^{o} / p\right)^{G_{n+1}} \\
& =\left(W^{o} / p W^{o}\right)^{G_{n+1}} \otimes_{\mathbb{F}_{p}} \mathscr{C}^{\mathrm{an}}\left(G_{n}, \mathbb{Q}_{p}\right)^{o} / p,
\end{aligned}
$$

where the last equality follows from Lemma 2.1.2. All maps are $G_{\mathbb{Q}_{p}}$-equivariant. Note that $\left(W^{o} / p W^{o}\right)^{G_{n+1}}$ is a finite-dimensional $\mathbb{F}_{p}$-vector space by the admissibility of the completed cohomology. Hence, the action of $G_{\mathbb{Q}_{p}}$ on $\tilde{H}^{i}\left(K^{p}, \mathbb{Q}_{p}\right)^{G-\text { an }, o} / p$ necessarily factors through a finite quotient of $G_{\mathbb{Q}_{p}}$.

Now we can apply Proposition 3.3.1 of [BC08] (with $G_{0}=G_{\mathbb{Q}_{p}}, H_{0}=G_{\mathbb{Q}_{p}\left(\mu_{p^{\infty}}\right)}$ ) to obtain the Sen operator on $\tilde{H}^{i}\left(K^{p}, \mathbb{Q}_{p}\right)^{G-a n} \widehat{\otimes}_{\mathbb{Q}_{p}} C$ except that $\tilde{H}^{i}\left(K^{p}, \mathbb{Q}_{p}\right)^{G-a n}$ is not finite-dimensional. To get around this, one can argue in a similar way to Section 3.3.1 by finding a dense subspace of $\tilde{H}^{i}\left(K^{p}, \mathbb{Q}_{p}\right)^{G \text {-an }}$ which can be written as a union of finite-dimensional $G_{\mathbb{Q}_{p}}$-invariant subspaces. For example, one can take the subspace of $\mathrm{GL}_{2}\left(\mathbb{Z}_{p}\right)$-algebraic vectors. Indeed, the density is clear when $i=0$. When $i=1$, the proof of Lemma 6.3 .8 implies that $\mathrm{GL}_{2}\left(\mathbb{Z}_{p}\right)$-algebraic vectors are dense in $\tilde{H}^{i}\left(K^{p}, \mathbb{Q}_{p}\right)$ and hence also dense in $\tilde{H}^{i}\left(K^{p}, \mathbb{Q}_{p}\right)^{G \text {-an }}$. Therefore, one gets the desired unique Sen operator on $\tilde{H}^{i}\left(K^{p}, \mathbb{Q}_{p}\right)^{G-\text { an }} \widehat{\otimes}_{\mathbb{Q}_{p}} C$. This defines the Sen operator on

$$
\underset{n}{\lim }\left(\tilde{H}^{i}\left(K^{p}, \mathbb{Q}_{p}\right)^{G_{n}-\mathrm{an}} \widehat{\otimes}_{\mathbb{Q}_{p}} C\right) \cong \tilde{H}^{i}\left(K^{p}, C\right)^{\mathrm{la}} .
$$

To see this isomorphism as LB-spaces, we claim that $\lim _{\longrightarrow n}\left(W^{G_{n}-\mathrm{an}} \widehat{\otimes}_{\mathbb{Q}_{p}} C\right) \cong\left(W \widehat{\otimes}_{\mathbb{Q}_{p}} C\right)^{\text {la }}$ for any admissible $\mathbb{Q}_{p}$-Banach representation $W$ of $G_{0}$. This is clear for $W=\mathscr{C}\left(G_{0}, \mathbb{Q}_{p}\right)$ because we even have $\left(W \widehat{\otimes}_{\mathbb{Q}_{p}} C\right)^{G_{n} \text {-an }} \cong W^{G_{n}-\text { an }} \widehat{\otimes}_{\mathbb{Q}_{p}} C$ in this case. The general case can be proved by embedding $W$ into $\mathscr{C}\left(G_{0}, \mathbb{Q}_{p}\right)^{\oplus d}$ for some $d$ and applying the acyclicity result Corollary 2.2.4. We omit the details here.

\section{2. n-cohomology (I)}

5.2.1. We start to compute the $\mathfrak{n}$-cohomology of $H^{1}\left(\mathscr{F} \ell, \mathcal{O}_{K^{p}}^{\text {la, }}\right.$ ). Since $\mathfrak{n}$ is 1 -dimensional, $H^{0}(\mathfrak{n}, \bullet)$ (respectively $\left.H^{1}(\mathfrak{n}, \bullet)\right)$ are the $\mathfrak{n}$-invariants (respectively $\mathfrak{n}$-coinvariants). Denote by $\mathcal{O}_{K^{p}}^{\text {la, }, \mathfrak{n}}$ (respec- 
tively $\left.\left(\mathcal{O}_{K^{p}}^{\mathrm{la}, \chi}\right)_{\mathfrak{n}}\right)$ the $\mathfrak{n}$-invariants (respectively $\mathfrak{n}$-coinvariants) of $\mathcal{O}_{K^{p}}^{\mathrm{la}, \chi}$. For the purpose of introduction, we assume $\chi(h) \neq 0$. Then $H^{0}\left(\mathscr{F} \ell, \mathcal{O}_{K^{p}}^{\text {la }, \chi}\right)=0$ and we have $B$-equivariant maps ${ }^{3}$

$$
\begin{gathered}
0 \rightarrow H^{1}\left(\mathscr{F} \ell, \mathcal{O}_{K^{p}}^{\mathrm{la}, \chi, \mathfrak{n}}\right) \rightarrow H^{1}\left(\mathscr{F} \ell, \mathcal{O}_{K^{p}}^{\mathrm{la}, \chi}\right)^{\mathfrak{n}} \rightarrow H^{0}\left(\mathscr{F} \ell,\left(\mathcal{O}_{K^{p}}^{\mathrm{la}, \chi}\right)_{\mathfrak{n}}\right) \otimes_{C} \mathfrak{n}^{*} \rightarrow 0, \\
H^{1}\left(\mathscr{F} \ell, \mathcal{O}_{K^{p}}^{\mathrm{la}, \chi}\right)_{\mathfrak{n}} \cong H^{1}\left(\mathscr{F} \ell,\left(\mathcal{O}_{K^{p}}^{\mathrm{la}, \chi}\right)_{\mathfrak{n}}\right) .
\end{gathered}
$$

Here $\mathfrak{n}^{*}=\operatorname{Hom}_{C}(\mathfrak{n}, C)$. We will compute $\mathcal{O}_{K^{p}}^{\mathrm{la}, \chi, \mathfrak{n}},\left(\mathcal{O}_{K^{p}}^{\mathrm{la}, \chi}\right)_{\mathfrak{n}}$ in this subsection.

5.2.2. For a weight $\chi$, we write $\chi\left(\left(\begin{array}{ll}a & 0 \\ 0 & d\end{array}\right)\right)=n_{1} a+n_{2} d$ for some $n_{1}, n_{2} \in C$ and sometimes identify $\chi$ with an ordered pair $\left(n_{1}, n_{2}\right) \in C^{2}$.

First we compute $\left(\mathcal{O}_{K^{p}}^{\text {la }, \chi}\right)_{\mathfrak{n}}$. It turns out that for generic $\chi$, this is essentially the space of overconvergent modular forms. We need some notation here. Let $\infty \in \mathscr{F} \ell$ be the point where $e_{1}$ vanishes. We can consider the fibre of $\mathcal{O}_{K^{p}}^{\mathrm{la}, \chi}$ at $\infty$ (as a sheaf of $\mathcal{O}_{\mathscr{F} \ell}$-modules); that is, $\mathcal{O}_{K^{p}}^{\mathrm{la}, \mathcal{Z}} / \mathrm{m}_{\infty} \mathcal{O}_{K^{p}}^{\mathrm{la}, \mathcal{X}}$. Here $\mathrm{m}_{\infty}$ denotes the ideal sheaf defined by $\infty$.

Definition 5.2.3. For a weight $\chi$, we define

$$
M_{\chi}^{\dagger}\left(K^{p}\right):=H^{0}\left(\mathscr{F} \ell, \mathcal{O}_{K}^{\mathrm{la}, \chi} / \mathrm{m}_{\infty} \mathcal{O}_{K^{p}}^{\mathrm{la}, \chi}\right)
$$

the fibre of $\mathcal{O}_{K^{p}}^{\mathrm{la}, \chi}$ at $\infty$, and call it the space of overconvergent modular forms of weight $\chi$ of tame level $K^{p}$. There are natural actions of $G_{\mathbb{Q}_{p}}$, the Borel subgroup $B$ and Hecke operators away from $p$ on this space.

5.2.4. To justify its name, we can compare this definition with other existing definitions of overconvergent modular forms in the literature [Kat73, CM98, Pi113, AIS14, CHJ17]. We will only focus on integral weights to illustrate the main difference here.

Let $k$ be an integer. We first introduce overconvergent modular forms with full level at $p$. Let $\Gamma\left(p^{n}\right)=1+p^{n} M_{2}\left(\mathbb{Z}_{p}\right)$. We define the canonical locus $\mathcal{X}_{K^{p} \Gamma\left(p^{n}\right), c} \subset \mathcal{X}_{K^{p}} \Gamma\left(p^{n}\right)$ as follows: using the integral model and the index of Igusa components in Theorem 13.7.6 of [KM85], $\mathcal{X}_{K^{p} \Gamma\left(p^{n}\right), c}$ is the tubular neighbourhood of the nonsingular points (= non-supersingular points) of irreducible components of indices $\left(\mathbb{Z} / p^{n}\right)^{2} \rightarrow \mathbb{Z} / p^{n},(1,0) \mapsto 0$. Equivalently, on the ordinary locus of these irreducible components, the canonical subgroup (of level $n$ ) corresponds to $(*, 0) \subset\left(\mathbb{Z} / p^{n}\right)^{2}$ under the level structure. Sections of $\omega^{k}$ on any strict neighbourhood of $\mathcal{X}_{K^{p}} \Gamma\left(p^{n}\right), c$ are called overconvergent modular forms of weight $k$ of level $K^{p} \Gamma\left(p^{n}\right)$, which we denote by $M_{k}^{\dagger}\left(K^{p} \Gamma\left(p^{n}\right)\right)$. Here a strict neighbourhood of $\mathcal{X}_{K^{p} \Gamma\left(p^{n}\right), c}$ means an open set containing the closure $\overline{\mathcal{X}}_{K^{p}} \Gamma\left(p^{n}\right), c$. Clearly, $M_{k}^{\dagger}\left(K^{p} \Gamma\left(p^{n}\right)\right)$ forms a direct system when $n$ varies.

Definition 5.2.5. Let $k \in \mathbb{Z}$. We define the space of overconvergent modular forms of weight $k$ of tame level $K^{p}$ as

$$
M_{k}^{\dagger}\left(K^{p}\right):=\underset{n}{\lim } M_{k}^{\dagger}\left(K^{p} \Gamma\left(p^{n}\right)\right)
$$

The Galois group $G_{\mathbb{Q}_{p}}$, Borel subgroup $B$ and Hecke operators away from $p$ act naturally on it.

Recall that classical overconvergent modular forms are defined as follows. Let $\Gamma_{1}\left(p^{n}\right)=\left\{\left(\begin{array}{ll}a & b \\ c & d\end{array}\right) \in\right.$ $\left.\mathrm{GL}_{2}\left(\mathbb{Z}_{p}\right) \mid a-1, d-1, c \in p^{n} \mathbb{Z}_{p}\right\}$. Then we have the canonical locus $\mathcal{X}_{K^{p} \Gamma_{1}\left(p^{n}\right), c} \subset \mathcal{X}_{K^{p} \Gamma_{1}\left(p^{n}\right)}$, which

\footnotetext{
${ }^{3}$ Again, these follow from some standard spectral sequences, but one can avoid them here. Using the cover $\left\{U_{1}, U_{2}\right\}$, we have the exact sequence $0 \rightarrow \mathcal{O}_{K^{p}}^{\mathrm{la}, \chi}\left(U_{1}\right) \oplus \mathcal{O}_{K^{p}}^{\mathrm{la}, \chi}\left(U_{2}\right) \rightarrow \mathcal{O}_{K^{p}}^{\mathrm{la}, \chi}\left(U_{12}\right) \rightarrow H^{1}\left(\mathscr{F} \ell, \mathcal{O}_{K^{p}}^{\mathrm{la}, \chi}\right) \rightarrow 0$. Our claim follows on applying $H^{i}(\mathrm{n}, \cdot)$ to this exact sequence.
} 
is defined as the image of $\mathcal{X}_{K^{p} \Gamma_{\left(p^{n}\right), c}}$ under the natural map $\mathcal{X}_{K^{p} \Gamma\left(p^{n}\right)} \rightarrow \mathcal{X}_{K^{p} \Gamma_{1}\left(p^{n}\right)}$. Using KatzMazur's integral model, this can also be defined as tubular neighbourhood of non-supersingular points of irreducible components whose ordinary points classify ordinary elliptic curves with level structure at $p$ given by the canonical subgroup (of level $n$ ). We define $M_{k}^{\dagger}\left(K^{p} \Gamma_{1}\left(p^{n}\right)\right.$ ) similarly as sections defined

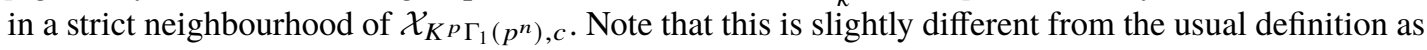
$\mathcal{X}_{K^{p} \Gamma_{1}\left(p^{n}\right)}$ is not connected.

We can obtain $M_{k}^{\dagger}\left(K^{p}\right)$ from $\lim _{\longrightarrow} M_{k}^{\dagger}\left(K^{p} \Gamma_{1}\left(p^{n}\right)\right)$ by inverting the action of $\left(\begin{array}{cc}p^{-1} & 0 \\ 0 & 1\end{array}\right)$. We can also recover $\lim _{\longrightarrow} M_{k}^{\dagger}\left(K^{p} \Gamma_{1}\left(p^{n}\right)\right)$ from $M_{k}^{\dagger}\left(K^{p}\right)$ by taking invariants of $N_{0}=\left(\begin{array}{cc}1 & \mathbb{Z}_{p} \\ 0 & 1\end{array}\right)$ :

$$
\underset{n}{\lim } M_{k}^{\dagger}\left(K^{p} \Gamma_{1}\left(p^{n}\right)\right)=M_{k}^{\dagger}\left(K^{p}\right)^{N_{0}}
$$

Proposition 5.2.6. Suppose $\chi\left(\left(\begin{array}{ll}a & 0 \\ 0 & d\end{array}\right)\right)=n_{1} a+n_{2} d$ with $n_{1}, n_{2} \in \mathbb{Z}$. Let $k=\chi(h)=n_{1}-n_{2}$. There is $a$ canonical isomorphism induced by multiplication by $e_{2}^{-k} t^{n_{1}}$ :

$$
\phi_{\chi}: M_{k}^{\dagger}\left(K^{p}\right) \stackrel{\sim}{\rightarrow} M_{\chi}^{\dagger}\left(K^{p}\right)
$$

satisfying

$$
\left(g_{1}, g_{2}\right) \cdot \phi_{\chi}(f)=\phi_{\chi}\left(\left(g_{1}, g_{2}\right) \cdot f\right) d^{-k} \varepsilon_{p}(a d)^{n_{1}} \varepsilon_{p}\left(g_{2}\right)^{n_{1}-k},
$$

$f \in M_{k}^{\dagger}\left(K^{p}\right), g_{1}=\left(\begin{array}{ll}a & b \\ 0 & d\end{array}\right) \in B, g_{2} \in G_{\mathbb{Q}_{p}}$. Recall that $\varepsilon_{p}: G_{\mathbb{Q}_{p}} \rightarrow \mathbb{Z}_{p}^{\times}$is the p-adic cyclotomic character and regarded as a character $\mathbb{Q}_{p}^{\times} \rightarrow \mathbb{Z}_{p}^{\times}$sending $x$ to $x|x|$ via local class field theory.

Remark 5.2.7. For a $B \times G_{\mathbb{Q}_{p}}$-representation $W$ and integers $i, j, k$, we write $W \cdot e_{1}^{i} e_{2}^{j} t^{k}$ to denote the twist of $W$ by the character sending $\left(\left(\begin{array}{ll}a & b \\ 0 & d\end{array}\right), g\right) \in B \times G_{\mathbb{Q}_{p}}$ to $a^{i} d^{j} \varepsilon_{p}(a d)^{k} \varepsilon_{p}(g)^{i+j+k}$. Therefore, we can rewrite the isomorphism in Proposition 5.2.6 as

$$
M_{k}^{\dagger}\left(K^{p}\right) \cdot e_{2}^{-k} t^{n_{1}} \cong M_{\chi}^{\dagger}\left(K^{p}\right)
$$

Proof. For any $U \in \mathfrak{B}$, a neighbourhood of $\infty$ not containing the zero of $x$, since $e_{1}$ is not a basis now, as in Section 5.1.12, we can use $e_{2}$ instead. More precisely, let $y=\frac{1}{x}=\frac{e_{1}}{e_{2}}$. We can find $y_{n}, e_{2, n}, t_{n}$ as in Section 4.3.5 and define $\mathcal{O}^{n}(U)\left\{y, e_{2}, t\right\}$. Denote by $\mathcal{O}^{n}(U)\{y\} \subset \mathcal{O}^{n}(U)\left\{y, e_{2}, t\right\}$ the subset on which $\theta_{\mathfrak{h}}$ acts by zero. Then $\mathcal{O}_{K^{p}}^{\text {la, } \chi}(U)=\lim _{\longrightarrow} \mathcal{O}^{n}(U)\{y\} \cdot t^{n_{1}}\left(\frac{e_{2}}{e_{2, n}}\right)^{n_{2}-n_{1}}$. Equivalently, as in Lemma 5.1.2, any element in $\mathcal{O}^{n}(U)\{y\} \cdot t^{n_{1}}\left(\frac{e_{2}}{e_{2, n}}\right)^{n_{2}-n_{1}}$ can be written as

$$
t^{n_{1}}\left(\frac{e_{2}}{e_{2, n}}\right)^{n_{2}-n_{1}} \sum_{i \geq 0} c_{i}\left(y-y_{n}\right)^{i},
$$

where $c_{i} \in H^{0}\left(V_{G_{r(n)}}, \mathcal{O}_{\mathcal{X}_{K} p_{G_{r(n)}}}\right)$ with bound $\left\|c_{i}\right\| \leq C^{\prime} p^{(n-1) i}$ for a uniform $C^{\prime}$. There is no need to put $t_{n}^{n_{1}}$ here because $n_{1}$ is an integer.

We can take $G_{r(n)}$ to be some $\Gamma\left(p^{m}\right)$. We claim

- $V_{G_{r(n)}}$ is a strict neighbourhood of $\mathcal{X}_{K^{p} G_{r(n)}, c}$.

Since both are affinoid subsets, it is enough to check that $V_{G_{r(n)}}$ contains the closure of the non-cusp classical points of $\mathcal{X}_{K^{p} G_{r(n)}, c}$. We check this relation for their preimages in $\mathcal{X}_{K^{p}}$. Note that $U$ is a 
$G_{r(n)}$-invariant open neighbourhood of $\infty$. In particular, $U$ contains $G_{r(n)} \cdot \infty$ and $V_{\infty}$ contains the closed set $\pi_{\mathrm{HT}}^{-1}\left(G_{r(n)} \cdot \infty\right)$. Our claim follows from the following lemma.

Lemma 5.2.8. The preimages of non-cusp points of $\mathcal{X}_{K^{p}} \Gamma_{\left(p^{m}\right), c}$ in $\mathcal{X}_{K^{p}}$ map to $\Gamma\left(p^{m}\right) \cdot \infty$ under the Hodge-Tate period map for any $m \geq 1$.

Proof. If $m=1$, this follows from Lemma III.3.14 of [Sch15] by noting our canonical locus $\mathcal{X}_{K^{p} \Gamma(p), c}$ does not intersect the anticanonical locus in the reference. The general case $m \geq 2$ can be reduced to this case by using the action of $\left(\begin{array}{cc}p^{m-1} & 0 \\ 0 & 1\end{array}\right)$.

Now fix such a $U$. Note that $\infty$ is defined by $y=0$. We need to construct an isomorphism

$$
\mathcal{O}_{K^{p}}^{\mathrm{la}, \chi}(U) /(y) \stackrel{\sim}{\rightarrow} \underset{n}{\lim } M_{k}^{\dagger}\left(K^{p} \Gamma\left(p^{n}\right)\right) .
$$

Since $\mathcal{O}^{n}(U)\{y\} \cong \mathcal{O}_{V_{G_{r(n)}}^{+}}\left(V_{G_{r(n)}}\right)\left[\left[p^{-(n-1)}\left(y-y_{n}\right)\right]\right] \otimes_{\mathbb{Z}_{p}} \mathbb{Q}_{p}$, there is a natural $\mathcal{O}_{V_{G_{r(n)}}}\left(V_{G_{r(n)}}\right)$ algebra homomorphism

$$
\varphi_{n}: \mathcal{O}^{n}(U)\{y\} /(y) \rightarrow \mathcal{O}_{V_{G_{r(n)}}}\left(V_{G_{r(n)}^{\prime \prime}}^{\prime \prime}\right)
$$

sending $p^{-(n-1)}\left(y-y_{n}\right)$ to $-p^{-(n-1)} y_{n} \in \mathcal{O}_{V_{G_{r(n)}}}\left(V_{G_{r(n)}^{\prime \prime}}^{\prime \prime}\right)$, where $V_{G_{r(n)}^{\prime \prime}}^{\prime \prime} \subset V_{G_{r(n)}}$ is the rational subset defined by $\left|p^{-n} y_{n}\right| \leq 1$. The same argument as above shows that $V_{G_{r(n)}^{\prime \prime}}^{\prime \prime}$ is a strict neighbourhood of $\mathcal{X}_{K^{p} G_{r(n)}, c}$. Let $V_{G_{r(n)}}^{\prime} \subset V_{G_{r(n)}}$ defined by $\left|p^{-(n-1)} y_{n}\right| \leq 1$. We claim that

$\circ$ the image of $\varphi_{n}$ contains analytic functions convergent on $V_{G_{r(n)}}^{\prime}$.

Indeed, since $\mathcal{O}_{V_{G_{r}(n)}}\left(V_{G_{r(n)}}^{\prime}\right) \cong \mathcal{O}_{V_{G_{r(n)}}}\left(V_{G_{r(n)}}\right)\left\langle p^{-(n-1)} y_{n}\right\rangle$, this claim is clear in view of the definition of $\varphi_{n}$.

Hence, we have a map

$$
\mathcal{O}^{n}(U)\{y\} \cdot t^{n_{1}}\left(\frac{e_{2}}{e_{2, n}}\right)^{n_{2}-n_{1}} /(y) \rightarrow \omega^{k}\left(V_{G_{r(n)}^{\prime \prime}}^{\prime \prime}\right)
$$

by sending $f t^{n_{1}}\left(\frac{e_{2}}{e_{2, n}}\right)^{n_{2}-n_{1}}$ to $\varphi_{n}(f) e_{2, n}^{n_{1}-n_{2}}$; that is, multiplication by $t^{-n_{1}} e_{2}^{n_{1}-n_{2}}$. Clearly, this map is compatible when $n$ varies and induces a map $\mathcal{O}_{K^{p}}^{\mathrm{la}, \chi}(U) /(y) \rightarrow \underset{\lim _{\longrightarrow}}{\longrightarrow} M_{k}^{\dagger}\left(K^{p} \Gamma\left(p^{n}\right)\right)$.

To prove this is an isomorphism, it suffices to show that for any $n$ and strict neighbourhood $W$ of $\mathcal{X}_{K^{p} \Gamma\left(p^{n}\right), c}$, we can find $m \geq n$ such that the preimage of $W$ in $\mathcal{X}_{K^{p} G_{r(m)}}$ contains $V_{G_{r(m)}}^{\prime}$. Let $\tilde{W}$ be the preimage of $W$ in $\mathcal{X}_{K^{p}}$. Then it is an open neighbourhood of $\pi_{\mathrm{HT}}^{-1}(\infty)=\left\{x \in \mathcal{X}_{K^{p}}|| y(x) \mid=0\right\}$. Therefore, $\left\{x \in \mathcal{X}_{K^{p}}|| p^{-m} y(x) \mid \geq 1\right\}, m=0,1, \cdots$ and $\tilde{W}$ form an open cover of $V_{\infty}$. Note that $V_{\infty}$ is quasi-compact because it is affinoid; hence, $\left\{z \in V_{\infty}|| p^{-(m-1)} y(z) \mid \leq 1\right\} \subset \tilde{W}$ for some $m$. Using $\left\|y-y_{m}\right\| \leq p^{-m}$ on $V_{\infty}$, it is easy to see that this $m$ works here.

The claim for the $B$-action follows from the construction and the fact that

$$
\left(\begin{array}{ll}
a & b \\
0 & d
\end{array}\right) \cdot e_{2}=b e_{1}+d e_{2}
$$

whose reduction modulo $\mathfrak{m}_{\infty}$ is $d e_{2}$ and that $\left(\begin{array}{ll}a & b \\ 0 & d\end{array}\right) \cdot t=a d|a d|$. The claim for $G_{\mathbb{Q}_{p}}$-action is clear as $G_{\mathbb{Q}_{p}}$ acts via the cyclotomic character on $e_{2}, t$.

We record the following result obtained in this proof. 
Lemma 5.2.9. Given an open subset $U$ of $\mathscr{F} \ell$ containing $\infty$, there exists a strict neighbourhood of $\mathcal{X}_{K^{p} \Gamma\left(p^{n}\right), c}$ for some $n$ whose preimage in $\mathcal{X}_{K^{p}}$ is contained in $\pi_{\mathrm{HT}}^{-1}(U)$. Conversely, for any $n>0$ and any strict neighbourhood $W$ of $\mathcal{X}_{K^{p}} \Gamma\left(p^{n}\right), c$, there exists an open subset of $\mathscr{F} \ell$ containing $\infty$ whose preimage in $\mathcal{X}_{K^{p}}$ is contained in the preimage of $W$.

Proof. For the first claim, we may assume $U \in \mathfrak{B}$. Then this was proved around Lemma 5.2.8. The second claim follows from the argument in the second to last paragraph of the proof of Theorem 5.2.6.

We are going to compare $M_{\chi}^{\dagger}\left(K^{p}\right)$ with the $\mathfrak{n}$-coinvariants $\left(\mathcal{O}_{K^{p}}^{\mathrm{la}, \chi}\right)_{\mathfrak{n}}$ of $\mathcal{O}_{K^{p}}^{\mathrm{la}, \chi}$. Note that there is a natural action of $\mathfrak{h}$ on $\left(\mathcal{O}_{K^{p}}^{\text {la }, \chi}\right)_{\mathfrak{n}}$ induced from the action of $\mathfrak{b}$. We will always use this constant $\mathfrak{h}$-action from now on, unless otherwise specified (to distinguish with the horizontal action $\left.\theta_{\mathfrak{h}}\right)$. Since $M_{\mathcal{\chi}}^{\dagger}\left(K^{p}\right)$ is the fibre of $\mathcal{O}_{K^{p}}^{\mathrm{la}, \chi}$ at $\infty$, the action of $\mathfrak{b}$ on it factors through $\mathfrak{h}$. It is easy to see that

$\circ \mathfrak{h}$ acts on $M_{\chi}^{\dagger}\left(K^{p}\right)$ via $\chi$.

We denote by $i_{\infty}$ the natural embedding $\infty \hookrightarrow \mathscr{F} \ell$.

Proposition 5.2.10. Let $\chi=\left(n_{1}, n_{2}\right)$ be a weight.

(1) $\left(\mathcal{O}_{K^{p}}^{\mathrm{la}, \chi}\right)_{\mathfrak{n}}$ is a skyscraper sheaf supported at $\infty$ and hence has no $H^{1}$.

(2) $\mathfrak{n}\left(\mathcal{O}_{K^{p}}^{\mathrm{la}, \chi}\right) \subset \mathfrak{m}_{\infty} \mathcal{O}_{K^{p}}^{\mathrm{la}, \chi}$. Thus, we get a natural exact sequence

$$
0 \rightarrow\left(i_{\infty}\right)_{*} N^{\chi} \rightarrow\left(\mathcal{O}_{K^{p}}^{\mathrm{la}, \chi}\right)_{\mathfrak{n}} \rightarrow\left(i_{\infty}\right)_{*} M_{\chi}^{\dagger}\left(K^{p}\right) \rightarrow 0
$$

for some $N^{\chi}$. Moreover, $\mathfrak{h}$ acts on $N^{\chi}$ via $\left(n_{2}+1, n_{1}-1\right)$. In particular, this exact sequence splits naturally if $\chi(h) \neq 1$; that is, $\left.\chi\right|_{\mathfrak{h}_{0}} \neq\left.\rho\right|_{\mathfrak{h}_{0}}$. Recall that $\rho$ denotes the half-sum of positive roots.

(3) $\left(\mathcal{O}_{K}^{\mathrm{la}, \chi}\right)_{\mathfrak{n}}=\left(i_{\infty}\right)_{*} M_{\chi}^{\dagger}\left(K^{p}\right)$; that is, $N^{\chi}=0$ if $\chi(h) \neq 0,-1,-2, \cdots$ and is p-adically non-Liouville (see Remark 5.2.11 for a definition).

(4) $\left(\mathcal{O}_{K^{p}}^{\mathrm{la}, \chi}\right)_{\mathfrak{n}} \cong\left(i_{\infty}\right)_{*} M_{\chi}^{\dagger}\left(K^{p}\right) \oplus\left(i_{\infty}\right)_{*} M_{\chi}^{\dagger}\left(K^{p}\right) \cdot\left(e_{1} / e_{2}\right)^{1-\chi(h)}$ if $\chi(h) \in\{0,-1,-2, \cdots\}$.

Remark 5.2.11. Recall that according to Clark [Cla66], we say $\alpha \in C$ is $p$-adically non-Liouville if

$$
\lim \inf _{n \rightarrow \infty}|\alpha+n|^{1 / n}>0
$$

that is, it cannot be well approximated by rational integers. It appears naturally in the study of $p$-adic differential equations. Suppose $\alpha \notin \mathbb{Z}$ and consider the inhomogeneous differential equation around $x=0$,

$$
\left(x \frac{d}{d x}+\alpha\right) y=\frac{1}{1-x} .
$$

It has a unique formal power series solution $\sum_{n \geq 0} \frac{1}{n+\alpha} x^{n}$, whose convergence is equivalent with $\alpha$ being non-Liouville. In fact, it will be clear to the reader that a variant of this differential equation will show up in our case.

Proof. Let $U \in \mathfrak{B}$. We first show $\left(\mathcal{O}_{K^{p}}^{\text {la }, \chi}(U)\right)_{\mathfrak{n}}=0$ if $e_{1}$ is a basis on $U$. Clearly, this implies that $\left(\mathcal{O}_{K^{p}}^{\text {la, } \chi}\right)_{\mathfrak{n}}$ is supported at $\infty$. On such a $U$, using the notation in Lemma 5.1.2, any element in $f \in \mathcal{O}_{K^{p}}^{\mathrm{la}, \chi}(U)$ can be written as

$$
f=\left(\frac{t}{t_{N}}\right)^{n_{1}}\left(\frac{e_{1}}{e_{1, N}}\right)^{n_{2}-n_{1}} \sum_{i \geq 0} c_{i}^{(n)}(f)\left(x-x_{n}\right)^{i} .
$$

Since $u^{+} \in \mathfrak{n}$ acts trivially on $e_{1}, t$ and acts as the usual derivation on $\left(x-x_{n}\right)$, an argument similar to the proof of Lemma 5.1.2 shows that $\mathfrak{n}$ is a surjection on $\mathcal{O}_{K^{p}}^{\mathrm{la}, \chi}(U)$. 
Now let $U \in \mathfrak{B}$ containing $\infty$ and on which $e_{2}$ is a basis. As in the proof of Proposition 5.2.6, any $f \in \mathcal{O}_{K^{p}}^{\mathrm{la}, \chi}(U)$ can be written as

$$
f=\left(\frac{t}{t_{N}}\right)^{n_{1}}\left(\frac{e_{2}}{e_{2, N}}\right)^{n_{2}-n_{1}} \sum_{i \geq 0} c_{i}\left(y-y_{n}\right)^{i}
$$

for some $n$ and $c_{i}$. Using $u^{+} \cdot e_{2}=e_{1}=y e_{2}$ and $u^{+} \cdot y=-y^{2}$, we get

$$
\begin{aligned}
u^{+} \cdot f & =y\left(\frac{t}{t_{N}}\right)^{n_{1}}\left(\frac{e_{2}}{e_{2, N}}\right)^{n_{2}-n_{1}}\left(\sum_{i \geq 0}\left(n_{2}-n_{1}\right) c_{i}\left(y-y_{n}\right)^{i}-y \sum_{i \geq 1} i c_{i}\left(y-y_{n}\right)^{i-1}\right) \\
& =y\left(\frac{t}{t_{N}}\right)^{n_{1}}\left(\frac{e_{2}}{e_{2, N}}\right)^{n_{2}-n_{1}} \sum_{i \geq 0}\left(\left(n_{2}-n_{1}-i\right) c_{i}-(i+1) c_{i+1} y_{n}\right)\left(y-y_{n}\right)^{i} \in y \mathcal{O}_{K^{p}}^{\mathrm{la}, \chi}(U) .
\end{aligned}
$$

Hence, $\mathfrak{n}\left(\mathcal{O}_{K^{p}}^{\mathrm{la}, \chi}\right) \subset \mathfrak{m}_{\infty} \mathcal{O}_{K^{p}}^{\mathrm{la}, \chi}$ and we can define $N^{\chi}$ as in the lemma. Moreover, one can compute

$$
\begin{aligned}
\left(h-\left(n_{2}-n_{1}+2\right)\right) \cdot(y f) & =y\left(\frac{t}{t_{N}}\right)^{n_{1}}\left(\frac{e_{2}}{e_{2, N}}\right)^{n_{2}-n_{1}}\left(h+2\left(n_{1}-n_{2}\right)\right) \cdot\left(\sum_{i \geq 0} c_{i}\left(y-y_{n}\right)^{i}\right) \\
& =-2 u^{+} \cdot f .
\end{aligned}
$$

Also, it is easy to check that $z$ acts on everything via $\chi(z)=n_{1}+n_{2}$. Hence, we conclude that $\mathfrak{h}$ acts via $\left(n_{2}+1, n_{1}-1\right)$ on $N^{\chi}$.

The last two claims require a bit more work. We denote by $U_{n} \subset U$ the rational subset defined by $\left|p^{-n} y\right| \leq 1$. Then $U_{n} \in \mathfrak{B}$ and contains $\infty$. Note that $\pi_{\mathrm{HT}}^{-1}\left(U_{n}\right)$ is also the preimage of $V_{G_{r(n)}^{\prime \prime}}^{\prime \prime}=\{x \in$ $\left.V_{G_{r(n)}},\left|y_{n}(x) p^{-n}\right| \leq 1\right\}$ because $\left\|y-y_{n}\right\| \leq p^{-n}$. For $n$ sufficiently large so that both $\left(\frac{t}{t_{n}}\right)^{n_{1}},\left(\frac{e_{2}}{e_{2, n}}\right)^{n_{2}-n_{1}}$ converge, we denote by $C_{n} \subset \mathcal{O}_{K^{p}}^{\text {la }}\left(U_{n}\right)$ the subset of elements of the form

$$
\left(\frac{t}{t_{n}}\right)^{n_{1}}\left(\frac{e_{2}}{e_{2, n}}\right)^{n_{2}-n_{1}} \sum_{i \geq 0} c_{i} y^{i}
$$

where $c_{i} \in H^{0}\left(V_{G_{r(n)}^{\prime \prime}}^{\prime \prime}, \mathcal{O}_{\mathcal{X}_{K} p_{G_{r(n)}}}\right)$ with bound $\left\|c_{i}\right\| \leq C^{\prime} p^{(n-1) i}$ for a uniform $C^{\prime}$. Note that as $\|y\| \leq p^{-n}$ in $H^{0}\left(\pi_{\mathrm{HT}}^{-1}\left(U_{n}\right), \mathcal{O}_{\mathcal{X}_{K} p}\right)$, we can choose $y_{n}=0$ here. As before, $p^{(n-1) i} c_{i}$ defines an isomorphism $C_{n} \cong\left(\prod_{i \geq 0} H^{0}\left(V_{G_{r(n)}^{\prime \prime}}^{\prime \prime}, \mathcal{O}_{\mathcal{X}_{K}{ }_{G_{r}(n)}}\right)\right) \otimes_{\mathbb{Z}_{p}} \mathbb{Q}_{p}$ and $C_{n}$ forms a direct system when $n$ varies. It is easy to see that

$$
\left.\left(\mathcal{O}_{K^{p}}^{\mathrm{la}, \chi}\right)_{\mathfrak{n}}=\left(i_{\infty}\right)_{*} \underset{n}{(\lim }\left(C_{n}\right)_{\mathfrak{n}}\right)
$$

Assume that $n_{1}-n_{2}=\chi(h) \neq 0,-1,-2, \cdots$ and is $p$-adically non-Liouville. For any $f \in C_{n}$ and $n$ sufficiently large, we need to show that $y f \in u^{+}\left(C_{m}\right)$ for some $m \geq n$. Write

$$
f=\left(\frac{t}{t_{n}}\right)^{n_{1}}\left(\frac{e_{2}}{e_{2, n}}\right)^{n_{2}-n_{1}} \sum_{i \geq 0} c_{i} y^{i}
$$

for some $c_{i} \in H^{0}\left(V_{G_{r(n)}}^{\prime \prime}, \mathcal{O}_{\mathcal{X}_{K}{ }^{G_{r}(n)}}\right)$ and $\left\|c_{i}\right\| \leq C^{\prime} p^{(n-1) i}$ for some $C^{\prime}$. By our assumption, we can find $m \geq n$ such that

$$
\left|n_{1}-n_{2}+i\right| \geq p^{-i(m-n)}
$$


for $i$ sufficiently large. Then $\left\|\frac{c_{i}}{-n_{1}+n_{2}-i}\right\| \leq C^{\prime} p^{(m-1) i}$ for $i$ large enough and

$$
\left(\frac{t}{t_{n}}\right)^{n_{1}}\left(\frac{e_{2}}{e_{2, n}}\right)^{n_{2}-n_{1}} \sum_{i \geq 0} \frac{c_{i}}{-n_{1}+n_{2}-i} y^{i}
$$

defines an element $g \in C_{m}$. A simple computation gives that $u^{+} \cdot g=y f$. Hence, $N^{\chi}=0$ and $\left(\mathcal{O}_{K^{p}}^{\text {la, }}\right)_{\mathfrak{n}}=\left(i_{\infty}\right)_{*} M_{\chi}^{\dagger}\left(K^{p}\right)$ in this case.

Now assume $n_{1}-n_{2}=\chi(h) \in\{0,-1,-2, \cdots\}$. Recall that for $f \in C_{n}$ as above, we have

$$
u^{+} \cdot f=\left(\frac{t}{t_{n}}\right)^{n_{1}}\left(\frac{e_{2}}{e_{2, n}}\right)^{n_{2}-n_{1}} \sum_{i \geq 0}\left(n_{2}-n_{1}-i\right) c_{i} y^{i+1} .
$$

Hence, the $y^{n_{2}-n_{1}+1}$ term in the summation always has zero coefficient. Conversely, if $f=$ $\left(\frac{t}{t_{n}}\right)^{n_{1}}\left(\frac{e_{2}}{e_{2, n}}\right)^{n_{2}-n_{1}} \sum_{i \geq 0} c_{i} y^{i} \in C_{n}$ with $c_{n_{2}-n_{1}}=0$, we have $\left|n_{2}-n_{1}-i\right| \geq p^{-i}$ for $i$ sufficiently large and $\left(\frac{t}{t_{n}}\right)^{n_{1}}\left(\frac{e_{2}}{e_{2, n}}\right)^{n_{2}-n_{1}} \sum_{0 \leq i \neq n_{2}-n_{1}} \frac{c_{i}}{n_{2}-n_{1}-i} y^{i}$ converges to an element $g \in C_{n+1}$. Again, it is easy to check that $y f=u^{+} \cdot g$. Thus, we can define an isomorphism

$$
M_{\chi}^{\dagger}\left(U^{p}\right) \stackrel{\sim}{\rightarrow} N^{\chi}
$$

by sending $f$ to $\tilde{f} y^{n_{2}-n_{1}+1}$, where $\tilde{f} \in \mathcal{O}_{K^{p}}^{\mathrm{la}, \chi}(U)$ is a lifting of $f \in \mathcal{O}_{K^{p}}^{\mathrm{la}, \chi}(U) /(y)$. Since $y=e_{1} / e_{2}$, this isomorphism becomes $B$-equivariant if we twist the left-hand side by $\left(e_{1} / e_{2}\right)^{1-n_{1}+n_{2}}$.

Next we compute $\mathcal{O}_{K^{p}}^{\mathrm{la}, \chi, n}$, the $\mathfrak{n}$-invariants of $\mathcal{O}_{K^{p}}^{\text {la, } \chi}$.

\section{Proposition 5.2.12.}

(1) Suppose $U$ does not contain $\infty$; that is, $e_{1}$ is a basis on $U$. Fix $N$ large enough so that $\left(\frac{t}{t_{N}}\right)^{n_{1}},\left(\frac{e_{1}}{e_{1, N}}\right)^{n_{2}-n_{1}}$ converge. Then any $f \in \mathcal{O}_{K^{p}}^{\text {la, } \chi, \mathfrak{n}}(U)$ can be written as

$$
f=\left(\frac{t}{t_{N}}\right)^{n_{1}}\left(\frac{e_{1}}{e_{1, N}}\right)^{n_{2}-n_{1}} c
$$

for some $n \geq N$ sufficiently large and $c \in H^{0}\left(V_{G_{r(n)}}, \mathcal{O}_{\mathcal{X}_{K^{p}} G_{r(n)}}\right)$.

(2) Suppose $e_{2}$ is a basis on $U \in \mathfrak{B}$ and $\chi(h)=n_{1}-n_{2} \in\{0,-1,-2, \cdots\}$. Fix $N$ large enough so that $\left(\frac{t}{t_{N}}\right)^{n_{1}}$ converges. Then any $f \in \mathcal{O}_{K^{p}}^{\mathrm{la}, \chi, \mathfrak{n}}(U)$ can be written as

$$
f=\left(\frac{t}{t_{N}}\right)^{n_{1}}\left(\frac{e_{2}}{e_{2, N}}\right)^{n_{2}-n_{1}} c y^{n_{2}-n_{1}}=\left(\frac{t}{t_{N}}\right)^{n_{1}}\left(\frac{e_{1}}{e_{2, N}}\right)^{n_{2}-n_{1}} c
$$

for some $n \geq N$ and $c \in H^{0}\left(V_{G_{r(n)}}, \mathcal{O}_{\mathcal{X}_{K} p_{G_{r(n)}}}\right)$.

(3) $\mathfrak{h}$ acts on $\mathcal{O}_{K^{p}}^{\mathrm{la}, \chi, \mathfrak{n}}(U)$ via $\left(n_{2}, n_{1}\right)$ for any open subset $U \subset \mathscr{F} \ell$.

(4) Suppose $\chi(h) \neq 0,-1,-2, \cdots$. Then

$$
\underset{U \ni \infty}{\lim } \mathcal{O}_{K^{p}}^{\mathrm{la}, \chi, \mathrm{n}}(U)=0
$$

that is, the stalk of $\mathcal{O}_{K^{p}}^{\mathrm{la}, \chi, \mathrm{n}}$ at $\infty$ is zero.

Remark 5.2.13. The overconvergent modular forms of weight $\chi$ introduced in [Pi113, AIS14, CHJ17] are essentially the stalk of $\mathcal{O}_{K^{p}}^{\text {la, }, \text { nt }}$ at a $\mathbb{Q}_{p}$-rational point of $\mathscr{F} \ell \backslash\{\infty\}$. For example, the $\infty$ in $[\mathrm{CHJ} 17$, Theorem 1.1] corresponds to the locus where $e_{2}=0$; that is, $x=0$ in our setup. 
Proof. For the first part, as in Lemma 5.1.2, any $f \in \mathcal{O}_{K^{p}}^{\text {la, } \chi}(U)$ can be written as

$$
f=\left(\frac{t}{t_{N}}\right)^{n_{1}}\left(\frac{e_{1}}{e_{1, N}}\right)^{n_{2}-n_{1}} \sum_{i \geq 0} c_{i}^{(n)}(f)\left(x-x_{n}\right)^{i}
$$

for some $n$ and $c_{i}^{(n)}(f)$ are unique for such a $n$. Since $u^{+}$acts as the usual derivation on $\left(x-x_{n}\right)$, we see that $u^{+} \cdot f=0$ if and only if $c_{i}^{(n)}(f)=0, i>0$. This proves the first part.

Suppose $\infty \in U$ and $e_{2}$ is a basis on $U$. As in the proof of Proposition 5.2.10, for any $f \in$ $\mathcal{O}_{K^{p}}^{\text {la, } \chi}(U)$, we can find $n$ so that $\left.f\right|_{U_{n}}$ can be written as $f=\left(\frac{t}{t_{N}}\right)^{n_{1}}\left(\frac{e_{2}}{e_{2, N}}\right)^{n_{2}-n_{1}} \sum_{i \geq 0} c_{i} y^{i}$ for some $c_{i} \in H^{0}\left(V_{G_{r(n)}^{\prime \prime}}^{\prime \prime}, \mathcal{O}_{\mathcal{X}_{K^{p}} G_{r(n)}}\right)$. Then

$$
u^{+} \cdot f=\left(\frac{t}{t_{N}}\right)^{n_{1}}\left(\frac{e_{2}}{e_{2, N}}\right)^{n_{2}-n_{1}} \sum_{i \geq 0}\left(n_{2}-n_{1}-i\right) c_{i} y^{i+1} .
$$

Hence, that $u^{+} \cdot f=0$ is equivalent with

$$
\left(n_{2}-n_{1}-i\right) c_{i}=0, i \geq 0 .
$$

This implies all $c_{i}=0$; that is, $\left.f\right|_{U_{n}}=0$ if $\chi(h)=n_{1}-n_{2} \neq 0,-1,-2, \cdots$. When $\chi(h) \in$ $\{0,-1,-2, \cdots\}$, we see that all $c_{i}=0$ except $i=n_{2}-n_{1}$ and

$$
\left.y\right|_{U_{n}}=\left(\frac{t}{t_{N}}\right)^{n_{1}}\left(\frac{e_{2}}{e_{2, N}}\right)^{n_{2}-n_{1}} c_{i} y^{n_{2}-n_{1}}=\left(\frac{t}{t_{N}}\right)^{n_{1}}\left(\frac{e_{1}}{e_{2, N}}\right)^{n_{2}-n_{1}} c_{i} .
$$

We have shown that $f$ on $U^{n}:=\left\{z \in U|| y(z) p^{-n} \mid \geq 1\right\}$ has the form $\left(\frac{t}{t_{N}}\right)^{n_{1}}\left(\frac{e_{1}}{e_{2, N}}\right)^{n_{2}-n_{1}} c_{i}^{\prime}$ for some

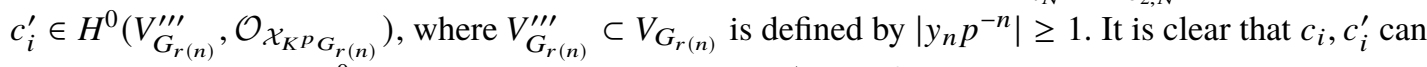
glue to a section $c \in H^{0}\left(V_{G_{r(n)}}, \mathcal{O}_{\mathcal{X}_{K} p_{G_{r(n)}}}\right)$ and $f=\left(\frac{t}{t_{N}}\right)^{n_{1}}\left(\frac{e_{1}}{e_{2, N}}\right)^{n_{2}-n_{1}} c$. This proves the second and fourth parts of the proposition.

The third part follows directly from the first two parts.

Remark 5.2.14. One can reinterpret these computations from the point of view of spectral theory. Consider the stalk of $\mathcal{O}_{K^{p}}^{\mathrm{la}, \chi}$ at $\infty$ :

$$
A^{\chi}:=\underset{U \ni \infty}{\lim } \mathcal{O}_{K^{p}}^{\mathrm{la}, \chi}(U)
$$

where the direct limit runs through all open subsets $U$ containing $\infty$. We equip $A^{\chi}$ with the direct limit topology. Note that if we fix $U \in \mathfrak{B}$ containing $\infty$ and $\left(\frac{t}{t_{n}}\right)^{n_{1}}\left(\frac{e_{2}}{e_{2, n}}\right)^{-\chi(h)} \in \mathcal{O}_{K^{p}}^{\text {la, } \chi}(U)$, then multiplication by $\left(\frac{t}{t_{n}}\right)^{-n_{1}}\left(\frac{e_{2}}{e_{2, n}}\right)^{\chi(h)}$ induces an isomorphism $A^{\chi} \stackrel{\sim}{\rightarrow} A^{0}$. Here 0 denotes the weight 0 . Clearly, this is not $\mathfrak{n}$-equivariant and a simple computation shows that the action of $u^{+}$on $A^{\chi}$ becomes

$$
u^{+}-\chi(h) y \text { on } A^{0}
$$

Now Proposition 5.2.10 and Proposition 5.2.12 can be rephrased as follows:

(1) $u^{+} / y$ is a well-defined operator on $A^{0}$.

(2) (outside of spectrum) $u^{+} / y-\chi(h)$ is invertible if $\chi(h) \neq 0,-1,-2, \cdots$ and is $p$-adically nonLiouville.

(3) (continuous spectrum) $u^{+} / y-\chi(h)$ is injective and has dense image if $\chi(h)$ is $p$-adically Liouville.

(4) (point spectrum) $u^{+} / y-\chi(h)$ has kernel the stalk of $\mathcal{O}_{K^{p}}^{\text {la, },}, \mathfrak{n}$ at $\infty$ if $-\chi(h) \in \mathbb{N}$,

summarising the results we have obtained so far. 
Theorem 5.2.15. Let $\chi=\left(n_{1}, n_{2}\right)$ be a weight. Then

(1) $\left(H^{1}\left(\mathscr{F} \ell, \mathcal{O}_{K}^{\mathrm{la}}\right)^{\chi}\right)_{\mathfrak{n}}=H^{1}\left(\mathscr{F} \ell, \mathcal{O}_{K^{p}}^{\mathrm{la}, \chi}\right)_{\mathfrak{n}}=0$ if $\chi(h) \neq 0$.

(2) Suppose $\chi(h) \neq 0,1$. There is a natural Hecke-equivariant weight decomposition of $C[B]$-modules

$$
H^{1}\left(\mathscr{F} \ell, \mathcal{O}_{K^{p}}^{\mathrm{la}}\right)^{\chi, \mathfrak{n}}=H^{1}\left(\mathscr{F} \ell, \mathcal{O}_{K^{p}}^{\mathrm{la}, \chi}\right)^{\mathfrak{n}}=M_{\chi}^{\dagger}\left(K^{p}\right) \otimes_{C} \mathfrak{n}^{*} \oplus W_{2},
$$

where $W_{2}$ has weight $\left(n_{2}, n_{1}\right)$ and $M_{\chi}^{\dagger}\left(K^{p}\right) \otimes_{C} \mathfrak{n}^{*}$ has weight $\left(n_{1}-1, n_{2}+1\right)$.

Proof. Assume $\chi(h) \neq 0$. Then by our discussion in the beginning of this subsection Equation (5.2.2), $H^{1}\left(\mathscr{F} \ell, \mathcal{O}_{K^{p}}^{\mathrm{la}, \chi}\right)_{\mathfrak{n}} \cong H^{1}\left(\mathscr{F} \ell,\left(\mathcal{O}_{K^{p}}^{\mathrm{la}, \chi}\right)_{\mathfrak{n}}\right)$, which is zero, as pointed out in Proposition 5.2.10.

Suppose $\chi(h) \neq 1$, then $-\chi(h) \neq w \cdot(-\chi)(h)$. It follows from Corollary 4.2.8 that $(h+\chi(h))(h-w$. $(-\chi)(h))=0$ on $H^{1}\left(\mathscr{F} \ell, \mathcal{O}_{K^{p}}^{\text {la, },}\right)^{\mathfrak{n}}$. Therefore, we have a natural weight decomposition $H^{1}\left(\mathscr{F} \ell, \mathcal{O}_{K^{p}}^{\text {la, } \chi}\right)^{\mathfrak{n}}=$ $W_{1} \oplus W_{2}$ such that $\mathfrak{h}$ acts on $W_{1}$ (respectively $\left.W_{2}\right)$ via $\left(n_{1}-1, n_{2}+1\right)$ (respectively $\left.\left(n_{2}, n_{1}\right)\right)$. Since $H^{1}\left(\mathscr{F} \ell, \mathcal{O}_{K^{p}}^{\text {la, }, \text { n }}\right)$ has no weight- $\left(n_{1}-1, n_{2}+1\right)$ part by Proposition 5.2.12 and the weight- $\left(n_{1}-1, n_{2}+1\right)$ part of $H^{0}\left(\mathscr{F} \ell,\left(\mathcal{O}_{K^{p}}^{\text {la, } \chi}\right)_{\mathfrak{n}}\right) \otimes_{C} \mathfrak{n}^{*}$ is $M_{\chi}^{\dagger}\left(K^{p}\right) \otimes_{C} \mathfrak{n}^{*}$ by Proposition 5.2.10, our claim for $W_{1}$ now follows from the short exact sequence (5.2.1).

The case $\chi(h)=0$ or 1 will be treated in the next subsection.

\section{3. n-cohomology (II)}

5.3.1. In this subsection, we completely determine the $\mathfrak{n}$-cohomology of $H^{1}\left(\mathscr{F} \ell, \mathcal{O}_{K^{p}}^{\text {la, } \chi}\right)$ for integral weight; that is, $\chi(h) \in \mathbb{Z}$. Write $k=\chi(h)$. We will distinguish 4 cases:

(1) $k \geq 2$;

(2) $k=1$;

(3) $k=0$;

(4) $k \leq-1$.

One key step is to understand $H^{1}\left(\mathscr{F} \ell, \mathcal{O}_{K^{p}}^{\text {la, }, \text {,n }}\right)$. To do this, we need to introduce some auxiliary sheaves. Recall that $\omega_{K^{p}}$ is defined as the pullback of $\omega$ (as a coherent sheaf) from some finite level to the infinite level. For an integer $k$, we write $\omega_{K^{p}}^{k}:=\omega_{K^{p}}^{\otimes k}$.

Definition 5.3.2. We define $\omega_{K^{p}}^{k \text {,sm }} \subset \pi_{\mathrm{HT} *} \omega_{K^{p}}^{k}$ as the subsheaf of $\mathrm{GL}_{2}\left(\mathbb{Q}_{p}\right)$-smooth sections. More precisely, for any quasi-compact open subset $U \subset \mathscr{F} \ell$, we can find an open subgroup $K_{p}$ of $\mathrm{GL}_{2}\left(\mathbb{Q}_{p}\right)$ stabilising $U$. Then $\omega_{K^{p}}^{k, \mathrm{sm}}(U) \subset \omega_{K^{p}}^{k}\left(\pi_{\mathrm{HT}}^{-1}(U)\right)$ is the subspace of $K_{p^{-}}$-smooth vectors; that is, vectors fixed by some open subgroup of $K_{p}$. It is easy to see that this definition is independent of the choice of $K_{p}$.

Remark 5.3.3. For $U \in \mathfrak{B}$, using the notation in Theorem 4.1.7, we have

$$
\omega_{K^{p}}^{k, \mathrm{sm}}(U)=\underset{K_{p}}{\lim } \omega^{k}\left(V_{K_{p}}\right) .
$$

Hence, compared with Proposition 5.2.12, it is clear that

$$
\omega_{K^{p}}^{0, \mathrm{sm}}=\mathcal{O}_{K^{p}}^{\mathrm{la}, 0, \mathfrak{n}}
$$

From the point of view of classical Riemann-Hilbert correspondence, it seems better to think of $\omega_{K^{p}}^{k \text {,sm }}$ as 'local system on the analytic site of $\mathscr{F} \ell$ '.

Remark 5.3.4. An equivalent definition is $\omega_{K^{p}}^{k, \mathrm{sm}}=\pi_{\mathrm{HT} *}\left(\lim _{K_{p} \subset \mathrm{GL}_{2}\left(\mathbb{Q}_{p}\right)}\left(\pi_{K_{p}}\right)^{-1} \omega^{k}\right)$. Here $\pi_{K_{p}}$ : $\mathcal{X}_{K^{p}} \rightarrow \mathcal{X}_{K^{p} K_{p}}$ denotes the natural projection and $\pi_{K_{p}}^{-1}$ denotes pullback as sheaf of abelian groups. In fact, the cohomology of $\omega_{K^{p}}^{k \text {,sm }}$ is closely related to the cohomology of $\omega^{k}$. 
Lemma 5.3.5. There is a natural isomorphism

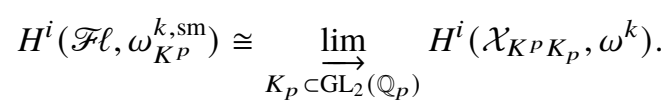

Proof. The rough idea is that the Hodge-Tate period map $\pi_{\mathrm{HT}}$ behaves as a finite map. Let $\mathfrak{U}=$ $\left\{U_{1}, \cdots, U_{r}\right\}$ be a subset of $\mathfrak{B}$ (not necessarily a cover of $\mathscr{F} \ell$ ); then for sufficiently small $K_{p}$ and any $i=1, \cdots, r$, we know that $\pi_{\mathrm{HT}}^{-1}\left(U_{i}\right)$ is the preimage of an affinoid open subset $V_{i, K_{p}} \subset \mathcal{X}_{K^{p}} K_{p}$. Denote

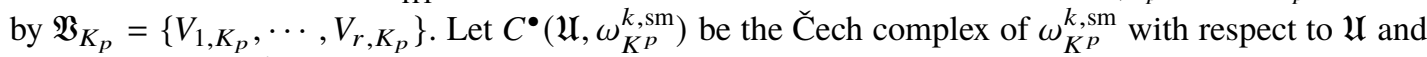
define $C^{\bullet}\left(\mathfrak{B}_{K_{p}}, \omega^{k}\right)$ in a similar way. Clearly,

$$
C^{\bullet}\left(\mathfrak{U}, \omega_{K^{p}}^{k, \mathrm{sm}}\right)=\underset{K_{p}}{\lim } C^{\bullet}\left(\mathfrak{B}_{K_{p}}, \omega^{k}\right) .
$$

In particular, if $\mathfrak{U}$ is an open cover of some $U \in \mathfrak{B}$, we conclude from the usual Tate acyclicity result that $H^{i}\left(C^{\bullet}\left(\mathfrak{H}, \omega_{K^{p}}^{k, \mathrm{sm}}\right)\right)=0, i \geq 1$. Hence, by Corollaire 4, p. 176 of [Gro57], we have $H^{i}\left(U, \omega_{K^{p}}^{k, \mathrm{sm}}\right)=0, i \geq 1$ and $H^{i}\left(\mathscr{F} \ell, \omega_{K^{p}}^{k, s m}\right)$ can be computed by Čech complex. Now taking $\mathfrak{U}$ as a cover of $\mathscr{F} \ell$, we get our claim.

Corollary 5.3.6. $H^{1}\left(\mathscr{F} \ell, \omega_{K^{p}}^{k, s m}\right)=0$ if $k \geq 2$.

Proof. By our previous lemma, it suffices to prove $H^{1}\left(\mathcal{X}_{K^{p} K_{p}}, \omega^{k}\right)=0$ when $k \geq 2$. This follows from the positivity of $\omega$ and the Kodaira-Spencer isomorphism $\omega^{2} \cong \Omega_{\mathcal{X}_{K^{p} K_{p}}}^{1}(\mathcal{C})$, where $\mathcal{C}$ denotes the cusps in $\mathcal{X}_{K^{p} K_{p}}$ and is nonempty.

The following lemma implies that the stalk of $\omega_{K^{p}}^{k, \text { sm }}$ at $\infty$ is the space of overconvergent forms of weight $k$ introduced in Definition 5.2.5.

Lemma 5.3.7. $\lim _{U \ni \infty} \omega_{K^{p}}^{k, \mathrm{sm}}(U)=M_{k}^{\dagger}\left(K^{p}\right)$.

Proof. This follows from Lemma 5.2.9 directly.

Definition 5.3.8. We denote by

$$
M_{k}\left(K^{p}\right):=H^{0}\left(\mathscr{F} \ell, \omega_{K^{p}}^{k, \mathrm{sm}}\right)=\underset{K_{p} \subset \overrightarrow{\mathrm{GL}}_{2}\left(\mathbb{Q}_{p}\right)}{\lim _{1}} H^{0}\left(\mathcal{X}_{K^{p}} K_{p}, \omega^{k}\right)
$$

the space of classical weight $k$ modular forms of tame level $K^{p}$.

5.3.9 (nonintegral powers of $t$ ). Recall that in Section 4.3.1, we defined $t$ as the coordinate function on $\operatorname{Isom}\left(\mathbb{Z}_{p}, \mathbb{Z}_{p}(1)\right) \cong \mathbb{Z}_{p}^{\times}$by choosing a basis of $\mathbb{Z}_{p}(1)$. For any $n_{1} \in C$, we can find a continuous character $\mathbb{Z}_{p}^{\times} \rightarrow C^{\times}$whose derivative sends $1 \in \mathbb{Q}_{p}=\operatorname{Lie}\left(\mathbb{Z}_{p}^{\times}\right)$to $n_{1} \in C=\operatorname{Lie}\left(C^{\times}\right)$. When $n_{1}$ is an integer, we can simply take $t^{n_{1}}$. For noninteger $n_{1}$, we fix one choice from now on and denote it by $t^{n_{1}}$ and view it as an invertible element of $H^{0}\left(\mathcal{X}_{K} p, \mathcal{O}_{\mathcal{X}_{K} p}\right)^{\text {la }}$ by abuse of notation. It follows from the discussion in Section 4.3.1 that $\mathrm{GL}_{2}\left(\mathbb{A}_{f}\right)$ acts on $t^{n_{1}}$ via $t^{n_{1}} \circ \varepsilon \circ \operatorname{det}$ and $G_{\mathbb{Q}_{p}}$ acts on it via $t^{n_{1}} \circ \varepsilon_{p}$. Hence, we can allow nonintegral power of $t$ in Remark 5.2.7.

Let $\chi=\left(n_{1}, n_{2}\right)$. Then multiplication by $t^{-n_{1}}$ induces an isomorphism $\mathcal{O}_{K^{p}}^{\text {la, } \chi} \stackrel{\sim}{\rightarrow} \mathcal{O}_{K^{p}}^{\text {la, } \chi^{\prime}}$, where $\chi^{\prime}=\left(0, n_{2}-n_{1}\right)$. It is easy to see how the actions of $\mathrm{GL}_{2}\left(\mathbb{Q}_{p}\right) \times G_{\mathbb{Q}_{p}}$ and Hecke operators away from $p$ change accordingly. From this, we can reduce computations to the case $n_{1}=0$.

5.3.10 $(k \geq 2)$. Now we are ready to treat the case $n_{1}-n_{2}=k \geq 2$. By Proposition 5.2.10, we have $\left(\mathcal{O}_{K^{p}}^{\text {la }, \chi}\right)_{\mathfrak{n}} \cong\left(i_{\infty}\right)_{*} M_{\chi}^{\dagger}\left(K^{p}\right)$ in this case and it suffices to determine $H^{1}\left(\mathscr{F} \ell, \mathcal{O}_{K^{p}}^{\text {la }, \chi}, \mathfrak{n}\right)$. Note that multiplication by $t^{-n_{1}} e_{1}^{k}$ induces an injection

$$
\mathcal{O}_{K^{p}}^{\mathrm{la}, \chi, \mathrm{n}} \rightarrow \omega_{K^{p}}^{k, \mathrm{sm}}
$$


More precisely, since the stalk of $\mathcal{O}_{K^{p}}^{\mathrm{la}, \boldsymbol{n}, \mathfrak{n}}$ at $\infty$ is zero, it is enough to define this map outside of $\infty$. So let $U \in \mathfrak{B}$ be an open subset not containing $\infty$. It follows from the first part of Proposition 5.2.12 that this map is nothing but sending $f=t^{n_{1}}\left(\frac{e_{1}}{e_{1, N}}\right)^{n_{2}-n_{1}} c \in \mathcal{O}_{K^{p}}^{\text {la, }, \text {,n }}(U)$ to $e_{1, N}^{k} c \in \omega_{K^{p}}^{k \text {,sm }}(U)$. This is, in fact, an isomorphism $\mathcal{O}_{K^{p}}^{\mathrm{la}, \chi}, \mathfrak{n}(U) \stackrel{\sim}{\rightarrow} \omega_{K^{p}}^{k, \mathrm{sm}}(U)$. Hence, the quotient of $\mathcal{O}_{K^{p}}^{\mathrm{la}, \chi, \mathfrak{n}} \rightarrow \omega_{K^{p}}^{k \text {,sm }}$ is simply the stalk of $\omega_{K^{p}}^{k, \mathrm{sm}}$ at $\infty$; that is,

$$
0 \rightarrow \mathcal{O}_{K^{p}}^{\mathrm{la}, \chi, \mathrm{n}} \stackrel{\times t^{-n_{1}} e_{1}^{k}}{\longrightarrow} \omega_{K^{p}}^{k, \mathrm{sm}} \rightarrow\left(i_{\infty}\right)_{*} M_{k}^{\dagger}\left(K^{p}\right) \rightarrow 0 .
$$

Take the cohomology of this exact sequence. It follows from Corollary 5.3.6 and Lemma 5.3.5 that

$$
0 \rightarrow M_{k}\left(K^{p}\right) \rightarrow M_{k}^{\dagger}\left(K^{p}\right) \rightarrow H^{1}\left(\mathscr{F} \ell, \mathcal{O}_{K^{p}}^{\mathrm{la}, \chi, \mathfrak{n}}\right) \rightarrow 0 .
$$

Hence, $H^{1}\left(\mathscr{F} \ell, \mathcal{O}_{K^{p}}^{\text {la, }, \text { nt }}\right) \cong M_{k}^{\dagger}\left(K^{p}\right) / M_{k}\left(K^{p}\right)$; that is, the quotient of overconvergent modular forms of weight $k$ by classical forms.

Theorem 5.3.11. Suppose $\chi(h)=k \in\{2,3, \cdots\}$. There is a Hecke and $B \times G_{\mathbb{Q}_{p}}$-equivariant weight decomposition (into weight $\left(n_{1}-1, n_{2}+1\right)$ and $\left(n_{2}, n_{1}\right)$ components):

$$
H^{1}\left(\mathscr{F} \ell, \mathcal{O}_{K^{p}}^{\mathrm{la}}\right)^{\chi, \mathfrak{n}}=H^{1}\left(\mathscr{F} \ell, \mathcal{O}_{K^{p}}^{\mathrm{la}, \mathcal{n}}\right)^{\mathfrak{n}}=M_{k}^{\dagger}\left(K^{p}\right) \cdot e_{1}^{-1} e_{2}^{-k+1} t^{n_{1}} \oplus\left(M_{k}^{\dagger}\left(K^{p}\right) / M_{k}\left(K^{p}\right)\right) \cdot e_{1}^{-k} t^{n_{1}} .
$$

Proof. We can multiply $t^{-n_{1}}$ to reduce to the case $n_{1}=0$. Assume $n_{1}=0$. Note that $\mathfrak{n}^{*}=e_{1}^{-1} e_{2}$ as $B$-representations. Hence, by Proposition 5.2.6, the weight $(-1,-k+1)$-part is isomorphic to $M_{k}^{\dagger}\left(K^{p}\right)$. $e_{1}^{-1} e_{2}^{-k+1}$. Since the isomorphism $H^{1}\left(\mathscr{F} \ell, \mathcal{O}_{K^{p}}^{\text {la, }, \mathfrak{n}}\right) \cong M_{k}^{\dagger}\left(K^{p}\right) / M_{k}\left(K^{p}\right)$ is essentially induced by $\times e_{1}^{k}$, we can twist by $e_{1}^{-k}$ to make it $B \times G_{\mathbb{Q}_{p}}$-equivariant.

5.3.12 $(k \leq-1)$. Another relatively simple case is when $\chi(h)=k \leq-1$. In this case, Proposition 5.2.12 implies that multiplication by $t^{-n_{1}} e_{1}^{k}$ induces an isomorphism

$$
\mathcal{O}_{K^{p}}^{\mathrm{la}, \chi, \mathfrak{n}} \stackrel{\sim}{\rightarrow} \omega_{K^{p}}^{k, \mathrm{sm}}
$$

Therefore, by Lemma 5.3.5, $H^{1}\left(\mathscr{F} \ell, \mathcal{O}_{K^{p}}^{\mathrm{la}, \chi, n}\right) \cong \lim _{K_{p}} H^{1}\left(\mathcal{X}_{K^{p} K_{p}}, \omega^{k}\right)$. Combining this with Proposition 5.2.10, we get the following.

Theorem 5.3.13. Suppose $\chi(h)=k \in\{-1,-2, \cdots\}$. There is a weight decomposition (into weight $\left(n_{1}-1, n_{2}+1\right)$ and $\left(n_{2}, n_{1}\right)$ components):

$$
H^{1}\left(\mathscr{F} \ell, \mathcal{O}_{K^{p}}^{\mathrm{la}, \chi}\right)^{\mathfrak{n}}=M_{k}^{\dagger}\left(K^{p}\right) \cdot e_{1}^{-1} e_{2}^{-k+1} t^{n_{1}} \oplus N_{k, 1} \cdot e_{1}^{-k} t^{n_{1}},
$$

where $N_{k, 1}$ sits inside the exact sequence

$$
0 \rightarrow \underset{K_{p}}{\lim _{\longrightarrow}} H^{1}\left(\mathcal{X}_{K^{p}} K_{p}, \omega^{k}\right) \rightarrow N_{k, 1} \rightarrow M_{k}^{\dagger}\left(K^{p}\right) \rightarrow 0 .
$$

All of the maps are Hecke and $B \times G_{\mathbb{Q}_{p}}$-equivariant.

5.3.14 $(k=0)$. Now we consider the case $\chi(h)=0$. As before, we can multiply by $t^{-n_{1}}$ and assume $n_{1}=0$; that is, $\chi=0$. First we determine $H^{1}\left(\mathscr{F} \ell, \mathcal{O}_{K^{p}}^{\mathrm{la}, 0}\right)^{\mathfrak{n}}$. Let $\mathcal{F}$ be $\mathfrak{n}\left(\mathcal{O}_{K^{p}}^{\mathrm{la}, 0}\right) \subset \mathcal{O}_{K^{p}}^{\mathrm{la}, 0}$. Hence, the composite

$$
H^{1}\left(\mathscr{F} \ell, \mathcal{O}_{K^{p}}^{\mathrm{la}, 0} \stackrel{u^{+}}{\longrightarrow} H^{1}(\mathscr{F} \ell, \mathcal{F}) \otimes \mathfrak{n}^{*} \rightarrow H^{1}\left(\mathscr{F} \ell, \mathcal{O}_{K^{p}}^{\mathrm{la}, 0}\right) \otimes \mathfrak{n}^{*}\right.
$$

is the endomorphism $u^{+}$on $H^{1}\left(\mathscr{F} \ell, \mathcal{O}_{K^{p}}^{\text {la, } 0}\right)$ and it suffices to determine the kernels of both maps. Consider

$$
0 \rightarrow \mathcal{O}_{K^{p}}^{\mathrm{la}, 0, \mathfrak{n}} \rightarrow \mathcal{O}_{K^{p}}^{\mathrm{la}, 0} \stackrel{u^{+}}{\rightarrow} \mathcal{F} \otimes \mathfrak{n}^{*} \rightarrow 0
$$


By Proposition 5.2.10, we know that $\mathcal{F} \subset \mathrm{m}_{\infty} \mathcal{O}_{K^{p}}^{\text {la, }}$. Hence, $H^{0}(\mathscr{F} \ell, \mathcal{F})=0$ because the global sections

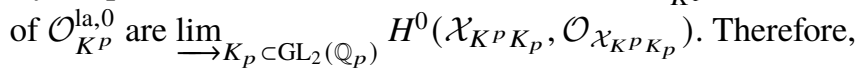

$$
H^{1}\left(\mathscr{F} \ell, \mathcal{O}_{K^{p}}^{\mathrm{la}, 0, \mathfrak{n}}\right)=\operatorname{ker}\left(H^{1}\left(\mathscr{F} \ell, \mathcal{O}_{K^{p}}^{\mathrm{la}, 0}\right) \stackrel{u^{+}}{\rightarrow} H^{1}(\mathscr{F} \ell, \mathcal{F}) \otimes \mathfrak{n}^{*}\right)
$$

and $H^{1}\left(\mathscr{F} \ell, \mathcal{O}_{K^{p}}^{\text {la, } 0}\right) \stackrel{u^{+}}{\longrightarrow} H^{1}(\mathscr{F} \ell, \mathcal{F}) \otimes \mathfrak{n}^{*}$ is surjective because $\mathscr{F} \ell$ is 1-dimensional. Note that $\mathcal{O}_{K^{p}}^{\text {la, } 0, \mathfrak{n}}=$ $\omega_{K^{p}}^{0, \text { sm }}$. Hence, its $H^{1} \underline{\lim }_{K_{p}} H^{1}\left(\mathcal{X}_{K^{p} K_{p}}, \mathcal{O}_{\mathcal{X}_{K^{p} K_{p}}}\right)$. On the other hand, consider

$$
0 \rightarrow \mathcal{F} \rightarrow \mathcal{O}_{K^{p}}^{\mathrm{la}, 0} \rightarrow\left(\mathcal{O}_{K^{p}}^{\mathrm{la}, 0}\right)_{\mathfrak{n}} \rightarrow 0 .
$$

By Proposition 5.2.10, $H^{0}\left(\mathscr{F} \ell,\left(\mathcal{O}_{K^{p}}^{\mathrm{la}, 0}\right)_{\mathfrak{n}}\right) \cong M_{0}^{\dagger}\left(K^{p}\right) \oplus M_{0}^{\dagger}\left(K^{p}\right) \otimes_{C} \mathfrak{n}$. It is easy to see that $H^{0}\left(\mathscr{F} \ell, \mathcal{O}_{K^{p}}^{\text {la, } 0}\right)=M_{0}\left(K^{p}\right)$ maps to the first factor. Hence,

$$
\operatorname{ker}\left(H^{1}(\mathscr{F} \ell, \mathcal{F}) \rightarrow H^{1}\left(\mathscr{F} \ell, \mathcal{O}_{K^{p}}^{\mathrm{la}, 0}\right)\right) \cong M_{0}^{\dagger}\left(K^{p}\right) / M_{0}\left(K^{p}\right) \oplus M_{0}^{\dagger}\left(K^{p}\right) \otimes_{C} \mathfrak{n} .
$$

Also, since $\left(\mathcal{O}_{K^{p}}^{\text {la, } 0}\right)_{\mathfrak{n}}$ has no $H^{1}$, we have $H^{1}(\mathscr{F} \ell, \mathcal{F}) \rightarrow H^{1}\left(\mathscr{F} \ell, \mathcal{O}_{K^{p}}^{\text {la, } 0}\right)$ is surjective. Summarising our discussions, we have the following result.

Proposition 5.3.15. Suppose $\chi=0$. Then

(1) $H^{1}\left(\mathscr{F} \ell, \mathcal{O}_{K^{p}}^{\mathrm{la}, 0}\right)_{\mathfrak{n}}=0$;

(2) there is a Hecke and $B \times G_{\mathbb{Q}_{p}}$-equivariant exact sequence:

$$
0 \rightarrow \underset{K_{p}}{\lim _{\longrightarrow}} H^{1}\left(\mathcal{X}_{K^{p} K_{p}}, \mathcal{O}_{\mathcal{X}_{K^{p} K_{p}}}\right) \rightarrow H^{1}\left(\mathscr{F} \ell, \mathcal{O}_{K^{p}}^{\mathrm{la}, 0}\right)^{\mathfrak{n}} \rightarrow \mathfrak{n}^{*} \otimes M_{0}^{\dagger}\left(K^{p}\right) / M_{0}\left(K^{p}\right) \oplus M_{0}^{\dagger}\left(K^{p}\right) \rightarrow 0 .
$$

By Corollary 5.1.3, there is an exact sequence

$$
0 \rightarrow M_{0}\left(K^{p}\right) \rightarrow H^{1}\left(\mathscr{F} \ell, \mathcal{O}_{K^{p}}^{\text {la, } 0}\right) \rightarrow H^{1}\left(\mathscr{F} \ell, \mathcal{O}_{K^{p}}^{\text {la }}\right)^{0} \rightarrow 0
$$

Note that $M_{0}\left(K^{p}\right)$ has trivial $\mathfrak{n}$-action. It is crucial to know its image in $H^{1}\left(\mathscr{F} \ell, \mathcal{O}_{K^{p}}^{\text {la, } 0}\right)^{\mathfrak{n}}$. A direct computation shows that the composite of

$$
M_{0}\left(K^{p}\right) \rightarrow H^{1}\left(\mathscr{F} \ell, \mathcal{O}_{K^{p}}^{\mathrm{la}, 0}\right)^{\mathfrak{n}} \rightarrow \mathfrak{n}^{*} \otimes M_{0}^{\dagger}\left(K^{p}\right) / M_{0}\left(K^{p}\right) \oplus M_{0}^{\dagger}\left(K^{p}\right)
$$

is the natural inclusion $M_{0}\left(K^{p}\right) \rightarrow M_{0}^{\dagger}\left(K^{p}\right)$ (up to a sign). Here the second map comes from the previous proposition. From this, taking the $\mathfrak{n}$-cohomology of the sequence (5.3.1), we obtain the following description in the case $\chi(h)=0$.

Theorem 5.3.16. Suppose $\chi=\left(n_{1}, n_{1}\right)$; that is, $k=0$. Then

(1) $\left(H^{1}\left(\mathscr{F} \ell, \mathcal{O}_{K}^{\mathrm{la}}\right)^{\chi}\right)_{\mathfrak{n}}=0$;

(2) there is a weight decomposition (into weight $\left(n_{1}-1, n_{1}+1\right)$ and $\left(n_{1}, n_{1}\right)$ components):

$$
H^{1}\left(\mathscr{F} \ell, \mathcal{O}_{K^{p}}^{\mathrm{la}}\right)^{\chi, \mathfrak{n}}=N_{2, w} \cdot e_{1}^{-1} e_{2} t^{n_{1}} \oplus N_{0,1} \cdot t^{n_{1}},
$$

where $N_{2, w}$ and $N_{0,1}$ sit inside exact sequences

$$
\begin{gathered}
0 \rightarrow M_{0}^{\dagger}\left(K^{p}\right) / M_{0}\left(K^{p}\right) \rightarrow N_{2, w} \rightarrow M_{0}\left(K^{p}\right) \rightarrow 0, \\
0 \rightarrow \underset{K_{p}}{\lim } H^{1}\left(\mathcal{X}_{K^{p} K_{p}}, \mathcal{O}_{\mathcal{X}_{K^{p} K_{p}}}\right) \rightarrow N_{0,1} \rightarrow M_{0}^{\dagger}\left(K^{p}\right) / M_{0}\left(K^{p}\right) \rightarrow 0 .
\end{gathered}
$$

All of the maps are Hecke and $B \times G_{\mathbb{Q}_{p}}$-equivariant. 
5.3.17 $(k=1)$. Finally, we treat the case of singular weight; that is, $\chi(h)=1$. Again we may assume $n_{1}=0$ by a twist. Note that by Proposition 5.2.10, we have $\left(\mathcal{O}_{K^{p}}^{\text {la }, \chi}\right)_{\mathfrak{n}}=\left(i_{\infty}\right)_{*} M_{\chi}^{\dagger}\left(K^{p}\right)$. Similar to the case $k \geq 2$ in 5.3.10, there is an exact sequence

$$
0 \rightarrow \mathcal{O}_{K^{p}}^{\mathrm{la}, \chi, \mathfrak{n}} \stackrel{\times e_{1}}{\longrightarrow} \omega_{K^{p}}^{1, \mathrm{sm}} \rightarrow\left(i_{\infty}\right)_{*} M_{1}^{\dagger}\left(K^{p}\right) \rightarrow 0
$$

induced by multiplication by $e_{1}$. The difference here is that $\omega_{K^{p}}^{1, \mathrm{sm}}$ have both nontrivial $H^{0}$ and $H^{1}$. More precisely, taking the cohomology of this exact sequence, we get

$$
0 \rightarrow M_{1}\left(K^{p}\right) \rightarrow M_{1}^{\dagger}\left(K^{p}\right) \rightarrow H^{1}\left(\mathscr{F} \ell, \mathcal{O}_{K^{p}}^{\mathrm{la}, \chi, \mathfrak{n}}\right) \rightarrow \underset{K_{p}}{\lim _{\longrightarrow}} H^{1}\left(\mathcal{X}_{K^{p}} K_{p}, \omega^{1}\right) \rightarrow 0 .
$$

Then by our discussion in Section 5.2.1, we have the following result.

Theorem 5.3.18. Suppose $\chi=\left(n_{1}, n_{1}-1\right)$; that is, $\chi(h)=1$. All of the maps below are Hecke and $B \times G_{\mathbb{Q}_{p}}$-equivariant.

(1) There is a short exact sequence

$$
0 \rightarrow N_{1} \cdot e_{1}^{-1} t^{n_{1}} \rightarrow H^{1}\left(\mathscr{F} \ell, \mathcal{O}_{K}^{\text {la }}\right)^{\chi, \mathfrak{n}} \rightarrow M_{1}^{\dagger}\left(K^{p}\right) \cdot e_{1}^{-1} t^{n_{1}} \rightarrow 0
$$

where $N_{1}$ sits inside the exact sequence

$$
0 \rightarrow M_{1}^{\dagger}\left(K^{p}\right) / M_{1}\left(K^{p}\right) \rightarrow N_{1} \rightarrow \underset{K_{p}}{\lim _{\longrightarrow}} H^{1}\left(\mathcal{X}_{K^{p} K_{p}}, \omega^{1}\right) \rightarrow 0 .
$$

(2) Under (5.3.2), the weight- $\left(n_{1}-1, n_{1}\right)$ part $H^{1}\left(\mathscr{F} \ell, \mathcal{O}_{K^{p}}^{\mathrm{la}}\right)_{\left(n_{1}-1, n_{1}\right)}^{\chi, \mathfrak{n}}$ of $H^{1}\left(\mathscr{F} \ell, \mathcal{O}_{K^{p}}^{\mathrm{la}}\right)^{\chi, \mathfrak{n}}$ is the pullback of $M_{1}\left(K^{p}\right) \cdot e_{1}^{-1} t^{n_{1}} \subset M_{1}^{\dagger}\left(K^{p}\right) \cdot e_{1}^{-1} t^{n_{1}}$; that is, it sits inside the exact sequence

$$
0 \rightarrow N_{1} \cdot e_{1}^{-1} t^{n_{1}} \rightarrow H^{1}\left(\mathscr{F} \ell, \mathcal{O}_{K}^{\mathrm{la}}\right)_{\left(n_{1}-1, n_{1}\right)}^{\chi, \mathrm{n}} \rightarrow M_{1}\left(K^{p}\right) \cdot e_{1}^{-1} t^{n_{1}} \rightarrow 0 .
$$

Proof. Only the second part requires extra explanation. To see this, we tensor the sequence (5.3.2) with $e_{1} t^{-n_{1}}$ and take $\mathfrak{h}_{0}$-cohomology. Recall that $\mathfrak{h}_{0} \subset \mathfrak{h}$ is the subalgebra of elements with trace zero. Again, we may assume $n_{1}=0$. We need to understand the kernel of the connecting homomorphism

$$
\delta_{1}: M_{1}^{\dagger}\left(K^{p}\right) \rightarrow\left(N_{1}\right)_{\mathfrak{h}_{0}} \cong N_{1} .
$$

Let $f \in M_{1}^{\dagger}\left(K^{p}\right)$. Since $H^{1}\left(\mathscr{F} \ell, \mathcal{O}_{K^{p}}^{\text {la }}\right)$ can be computed by Čech complex, we can take a cover $\left\{U_{0}^{\prime}, U_{1}^{\prime}\right\} \subset \mathfrak{B}$ of $\mathscr{F} \ell$ such that only $U_{1}^{\prime}$ contains $\infty$ and the pullback of $f$ to $\mathcal{X}_{K^{p}}$ is defined on $\pi_{\mathrm{HT}}^{-1}\left(U_{1}^{\prime}\right)$. On $U_{01}^{\prime}:=U_{0}^{\prime} \cap U_{1}^{\prime}$, we can find $g \in \mathcal{O}_{K^{p}}^{\mathrm{la}, \chi}\left(U_{01}^{\prime}\right)$ such that $u^{+} \cdot g=f e_{2}^{-1}$. Then $-g$ can be viewed as a 1-cocycle of the Čech complex of $\mathcal{O}_{K^{p}}^{\text {la, } \chi}$ with respect to the cover $\left\{U_{0}^{\prime}, U_{1}^{\prime}\right\}$, and it maps to $f \otimes 1 \in M_{1}^{\dagger}\left(K^{p}\right) \otimes e_{1}^{-1}=M_{1}^{\dagger}\left(K^{p}\right) \cdot e_{1}^{-1}$ in the sequence (5.3.2). (Here we view $e_{1}=C$ as a character of $B$.) Using the notation in Lemma 5.1.2, we can take $g$ of the form

$$
g=\frac{f}{e_{1}} \log \left(\frac{x}{x_{n}}\right) .
$$

If we view $g \otimes 1$ as an element of $\mathcal{O}_{K^{p}}^{\text {la, } \chi}\left(U_{01}^{\prime}\right) \otimes e_{1}$, then

$$
h \cdot(g \otimes 1)=h \cdot g \otimes 1+g \otimes 1=\left(-g \otimes 1-\frac{2 f}{e_{1}} \otimes 1\right)+g \otimes 1=-\frac{2 f}{e_{1}} \otimes 1 .
$$

In view of the discussion in Section 5.3.17, this means $\delta_{1}(f)=-2 f \in M_{1}^{\dagger}\left(K^{p}\right) / M_{1}\left(K^{p}\right) \subset N_{1}$. Hence, $\delta_{1}(f)=0$ if and only if $f \in M_{1}\left(K^{p}\right)$. From this, we easily deduce our claim in the Theorem. 


\section{4. $\mu$-isotypic part}

5.4.1. Let $\mu \in \mathfrak{h}^{*}$ be a weight, viewed as a character of $\mathfrak{b}$. The goal of this subsection is to determine the $\mu$-isotypic part of $\tilde{H}^{1}\left(K^{p}, C\right)^{\text {la }} \cong H^{1}\left(\mathscr{F} \ell, \mathcal{O}_{K^{p}}^{\text {la }}\right)$, which we denote by

$$
\tilde{H}^{1}\left(K^{p}, C\right)_{\mu}^{\mathrm{la}} \cong H^{1}\left(\mathscr{F} \ell, \mathcal{O}_{K}^{\mathrm{la}}\right)_{\mu} .
$$

Write $\mu=\left(k_{1}, k_{2}\right)$. Then by Corollary 4.2.8 and Harish-Chandra's theory, ${ }^{4}$ we have

$$
H^{1}\left(\mathscr{F} \ell, \mathcal{O}_{K^{p}}^{\text {la }}\right)_{\mu}=H^{1}\left(\mathscr{F} \ell, \mathcal{O}_{K^{p}}^{\text {la }}\right)_{\mu}^{\left(k_{2}, k_{1}\right)} \oplus H^{1}\left(\mathscr{F} \ell, \mathcal{O}_{K^{p}}^{\text {la }}\right)_{\mu}^{\left(k_{1}+1, k_{2}-1\right)}
$$

if $\left(k_{2}, k_{1}\right) \neq\left(k_{1}+1, k_{2}-1\right)$; that is, $\mu(h) \neq-1$. (This is opposite to the singular weight in the previous section as there is a sign in Corollary 4.2.8.) Since the right-hand side is computed in Theorems 5.2.15, 5.3.11, 5.3.13, 5.3.16 (which is complete in the integral weight case), we obtain the following theorem by writing

$$
M_{\mu, 1}:=H^{1}\left(\mathscr{F} \ell, \mathcal{O}_{K^{p}}^{\mathrm{la}}\right)_{\mu}^{\left(k_{2}, k_{1}\right)}, M_{\mu, w}:=H^{1}\left(\mathscr{F} \ell, \mathcal{O}_{K^{p}}^{\mathrm{la}}\right)_{\mu}^{\left(k_{1}+1, k_{2}-1\right)} .
$$

Note that by Theorem 5.1.11, the horizontal action $\theta_{\mathfrak{h}}$ of $\mathfrak{h}$ essentially agrees with the Sen operator. Hence, $M_{\mu, 1}$ has pure Hodge-Tate-Sen weight $-k_{1}$ and $M_{\mu, w}$ has pure Hodge-Tate-Sen weight $1-k_{2}$ in the sense of Definition 5.1.5. Our convention is that cyclotomic character has Hodge-Tate weight -1 .

Theorem 5.4.2. Suppose $\mu=\left(k_{1}, k_{2}\right) \in \mathfrak{h}^{*}$ and $k:=-\mu(h) \neq 1$. There is a natural (Hodge)decomposition

$$
\tilde{H}^{1}\left(K^{p}, C\right)_{\mu}^{\mathrm{la}}=M_{\mu, 1} \oplus M_{\mu, w}
$$

into Hodge-Tate-Sen weight $-k_{1}$ and $1-k_{2}$ components. We have $M_{\mu, w}=M_{\mu+2 \rho}^{\dagger}\left(K^{p}\right) \otimes_{C} \mathfrak{n}^{*}$ unless $k=2$. Moreover, if $k \in \mathbb{Z}$ and

$$
\begin{gathered}
M_{\mu, 1}=N_{k, 1} \cdot e_{1}^{-k} t^{k_{2}}, \\
M_{\mu, w}=N_{k, w} \cdot e_{1}^{-1} e_{2}^{k-1} t^{k_{1}+1},
\end{gathered}
$$

we have the following description of $N_{k, 1}, N_{k, w}$. All of the isomorphisms and maps below are Hecke and $B \times G_{\mathbb{Q}_{p}}$-equivariant.

(1) $N_{k, w} \cong M_{2-k}^{\dagger}\left(K^{p}\right)$ unless $k \neq 2$. When $k=2$, we have

$$
0 \rightarrow M_{0}^{\dagger}\left(K^{p}\right) / M_{0}\left(K^{p}\right) \rightarrow N_{2, w} \rightarrow M_{0}\left(K^{p}\right) \rightarrow 0 .
$$

(2) If $k \leq-1$, then there is an exact sequence

$$
0 \rightarrow \underset{K_{p}}{\lim _{\longrightarrow}} H^{1}\left(\mathcal{X}_{K^{p} K_{p}}, \omega^{k}\right) \rightarrow N_{k, 1} \rightarrow M_{k}^{\dagger}\left(K^{p}\right) \rightarrow 0 .
$$

(3) If $k=0$, then there is an exact sequence

$$
0 \rightarrow \underset{K_{p}}{\lim _{\longrightarrow}} H^{1}\left(\mathcal{X}_{K^{p} K_{p}}, \mathcal{O}_{\mathcal{X}_{K^{p} K_{p}}}\right) \rightarrow N_{0,1} \rightarrow M_{0}^{\dagger}\left(K^{p}\right) / M_{0}\left(K^{p}\right) \rightarrow 0 .
$$

(4) If $k \geq 2$, then $N_{k, 1} \cong M_{k}^{\dagger}\left(K^{p}\right) / M_{k}\left(K^{p}\right)$.

5.4.3. Now assume $\mu(h)=-1$. Write $\mu=\left(k_{1}, k_{1}+1\right)$. In this case, it follows from Corollary 4.2 .8 that

$$
H^{1}\left(\mathscr{F} \ell, \mathcal{O}_{K^{p}}^{\mathrm{la}}\right)_{\mu}=H^{1}\left(\mathscr{F} \ell, \mathcal{O}_{K^{p}}^{\mathrm{la},\left(k_{1}+1, k_{1}\right)^{2}}\right)_{\mu}
$$

\footnotetext{
${ }^{4}$ Note $w \cdot \mu=\left(k_{2}-1, k_{1}+1\right)$. Hence, we are looking for $(a, b) \in C$ such that $a+b=k_{1}+k_{2}$ and $-(a-b)=k_{1}-k_{2}$ or $\left(k_{2}-1\right)-\left(k_{1}+1\right)$.
} 
by considering the action of the centre of $U(\mathfrak{g})$. Here $\mathcal{O}_{K^{p}}^{\mathrm{la},\left(k_{1}+1, k_{1}\right)^{2}} \subset \mathcal{O}_{K^{p}}^{\mathrm{la}}$ is the subsheaf of sections annihilated by $\left(\theta_{\mathfrak{h}}(h)-1\right)^{2}$ and $\theta_{\mathfrak{h}}(z)-\mu(z)=z-\left(2 k_{1}+1\right)$. We compute the $\mathfrak{n}$-invariants of $H^{1}\left(\mathscr{F} \ell, \mathcal{O}_{K^{p}}^{\text {la, }\left(k_{1}+1, k_{1}\right)^{2}}\right)$ first and then determine the $\mu$-component. Note that $\theta_{\mathfrak{h}}(h)-1$ induces an exact sequence

$$
0 \rightarrow \mathcal{O}_{K^{p}}^{\mathrm{la},\left(k_{1}+1, k_{1}\right)} \rightarrow \mathcal{O}_{K^{p}}^{\mathrm{la},\left(k_{1}+1, k_{1}\right)^{2}} \stackrel{\theta_{\mathfrak{l}}(h)-1}{\longrightarrow} \mathcal{O}_{K^{p}}^{\mathrm{la},\left(k_{1}+1, k_{1}\right)} \rightarrow 0,
$$

where the third map is surjective by the first part of Lemma 5.1.2. For simplicity, we write $\mathcal{F}_{1}=$ $\mathcal{O}_{K^{p}}^{\text {la, }\left(k_{1}+1, k_{1}\right)}$ and $\mathcal{F}_{2}=\mathcal{O}_{K^{p}}^{\text {la, }\left(k_{1}+1, k_{1}\right)^{2}}$. Then $\mathcal{F}_{2}$ has no $H^{0}$ because $\mathcal{F}_{1}$ has no global section (Corollary 5.1.3). Hence,

$$
0 \rightarrow H^{1}\left(\mathscr{F} \ell,\left(\mathcal{F}_{2}\right)^{\mathfrak{n}}\right) \rightarrow H^{1}\left(\mathscr{F} \ell, \mathcal{F}_{2}\right)^{\mathfrak{n}} \rightarrow H^{0}\left(\mathscr{F} \ell,\left(\mathcal{F}_{2}\right)_{\mathfrak{n}}\right) \otimes_{C} \mathfrak{n}^{*} \rightarrow 0
$$

By Propositions 5.2.10 and 5.2.12, we know that the supports of $\left(\mathcal{F}_{1}\right)^{\mathfrak{n}}$ and $\left(\mathcal{F}_{1}\right)_{\mathfrak{n}}$ do not intersect. Therefore, $\theta_{\mathfrak{h}}(h)-1$ induces exact sequences

$$
\begin{gathered}
0 \rightarrow\left(\mathcal{F}_{1}\right)^{\mathfrak{n}} \rightarrow\left(\mathcal{F}_{2}\right)^{\mathfrak{n}} \stackrel{\theta_{\mathfrak{h}}(h)-1}{\longrightarrow}\left(\mathcal{F}_{1}\right)^{\mathfrak{n}} \rightarrow 0 ; \\
0 \rightarrow\left(\mathcal{F}_{1}\right)_{\mathfrak{n}} \rightarrow\left(\mathcal{F}_{2}\right)_{\mathfrak{n}} \stackrel{\theta_{\mathfrak{h}}(h)-1}{\longrightarrow}\left(\mathcal{F}_{1}\right)_{\mathfrak{n}} \rightarrow 0 .
\end{gathered}
$$

Taking $H^{1}$ of the first sequence, we obtain

$$
0 \rightarrow H^{1}\left(\mathscr{F} \ell,\left(\mathcal{F}_{1}\right)^{\mathfrak{n}}\right) \rightarrow H^{1}\left(\mathscr{F} \ell,\left(\mathcal{F}_{2}\right)^{\mathfrak{n}}\right) \stackrel{\theta_{\mathfrak{l}}(h)-1}{\longrightarrow} H^{1}\left(\mathscr{F} \ell,\left(\mathcal{F}_{1}\right)^{\mathfrak{n}}\right) \rightarrow 0
$$

as $\left(\mathcal{F}_{1}\right)^{\mathfrak{n}} \subset \mathcal{F}_{1}$ has no $H^{0}$. Note that $h$ acts as -1 on $H^{1}\left(\mathscr{F} \ell,\left(\mathcal{F}_{1}\right)^{\mathfrak{n}}\right)$ by Proposition 5.2.12.

Lemma 5.4.4. $\theta_{\mathfrak{h}}(h)-1=-(h+1)$ on $\left(\mathcal{F}_{2}\right)^{\mathfrak{n}}$. In particular, the kernel of $h+1$ on $H^{1}\left(\mathscr{F} \ell,\left(\mathcal{F}_{2}\right)^{\mathfrak{n}}\right)$ is $H^{1}\left(\mathscr{F} \ell,\left(\mathcal{F}_{1}\right)^{\mathfrak{n}}\right)$ and $h+1$ induces an isomorphism

$$
h+1: H^{1}\left(\mathscr{F} \ell,\left(\mathcal{F}_{2}\right)^{\mathfrak{n}}\right) / H^{1}\left(\mathscr{F} \ell,\left(\mathcal{F}_{1}\right)^{\mathfrak{n}}\right) \stackrel{\sim}{\rightarrow} H^{1}\left(\mathscr{F} \ell,\left(\mathcal{F}_{1}\right)^{\mathfrak{n}}\right) .
$$

Proof. This is proved by explicit computation. We only need to check $U \in \mathfrak{B}$ not containing $\infty$ since the stalk of $\left(\mathcal{F}_{1}\right)^{\mathfrak{n}}$, hence also $\left(\mathcal{F}_{2}\right)^{\mathfrak{n}}$, at $\infty$ is zero. Using the notation in Section 4.3.7, we have

$$
\left(\mathcal{F}_{2}\right)^{\mathfrak{n}}(U)=\left(\mathcal{F}_{1}\right)^{\mathfrak{n}}(U)+\left(\mathcal{F}_{1}\right)^{\mathfrak{n}}(U) \cdot \log \left(\frac{e_{1}}{e_{1, N}}\right)
$$

for some $e_{1, N}$. Since $\theta_{\mathfrak{h}}(h)$ acts as $\left(\begin{array}{cc}-1 & -2 x \\ 0 & 1\end{array}\right)$ on $U$ (cf. 5.1.1), for $f \in\left(\mathcal{F}_{1}\right)^{\mathfrak{n}}(U)$,

$$
\left(\theta_{\mathfrak{h}}(h)-1\right) \cdot\left(f \log \left(\frac{e_{1}}{e_{1, N}}\right)\right)=\log \left(\frac{e_{1}}{e_{1, N}}\right)\left(\theta_{\mathfrak{h}}(h)-1\right) \cdot f+f \theta_{\mathfrak{h}}(h) \cdot \log \left(\frac{e_{1}}{e_{1, N}}\right)=-f .
$$

On the other hand, by Proposition 5.2.12, we know that $(h+1) \cdot f=0$. Therefore,

$$
(h+1) \cdot\left(f \log \left(\frac{e_{1}}{e_{1, N}}\right)\right)=f h \cdot \log \left(\frac{e_{1}}{e_{1, N}}\right)=f .
$$

Hence, $\theta_{\mathfrak{h}}(h)-1=-(h+1)$ on $\left(\mathcal{F}_{2}\right)^{\mathfrak{n}}(U)$.

5.4.5. Similarly, we consider

$$
0 \rightarrow H^{0}\left(\mathscr{F} \ell,\left(\mathcal{F}_{1}\right)_{\mathfrak{n}}\right) \rightarrow H^{0}\left(\mathscr{F} \ell,\left(\mathcal{F}_{2}\right)_{\mathfrak{n}}\right) \stackrel{\theta_{\mathfrak{l}}(h)-1}{\longrightarrow} H^{0}\left(\mathscr{F} \ell,\left(\mathcal{F}_{1}\right)_{\mathfrak{n}}\right) \rightarrow 0
$$


Recall that $\left(\mathcal{F}_{1}\right)_{\mathfrak{n}} \cong\left(i_{\infty}\right)_{*} M_{\left(k_{1}+1, k_{1}\right)}^{\dagger}\left(K^{p}\right)$ in this case, and we may identify

$$
\left(\mathcal{F}_{2}\right)_{\mathfrak{n}}=\left(i_{\infty}\right)_{*}\left(M_{\left(k_{1}+1, k_{1}\right)}^{\dagger}\left(K^{p}\right) \oplus M_{\left(k_{1}+1, k_{1}\right)}^{\dagger}\left(K^{p}\right) \cdot \log \left(\frac{e_{2}}{e_{2, N}}\right)\right)
$$

for some $e_{2, N}$. Then a similar computation shows that $\theta_{\mathfrak{h}}(h)-1=h-1$ on $\left(\mathcal{F}_{2}\right)_{\mathfrak{n}}$. Hence, the kernel of $h+1$ on $H^{0}\left(\mathscr{F} \ell,\left(\mathcal{F}_{2}\right)_{\mathfrak{n}}\right) \otimes_{C} \mathfrak{n}^{*}$ is $H^{0}\left(\mathscr{F} \ell,\left(\mathcal{F}_{1}\right)_{\mathfrak{n}}\right) \otimes_{C} \mathfrak{n}^{*} \subset H^{0}\left(\mathscr{F} \ell,\left(\mathcal{F}_{2}\right)_{\mathfrak{n}}\right) \otimes_{C} \mathfrak{n}^{*}$.

Now to determine the kernel of $h+1$ on $H^{1}\left(\mathscr{F} \ell, \mathcal{F}_{2}\right)^{\mathfrak{n}}$, we claim that the surjection

$$
H^{1}\left(\mathscr{F} \ell, \mathcal{F}_{2}\right)^{\mathfrak{n}} \rightarrow H^{0}\left(\mathscr{F} \ell,\left(\mathcal{F}_{2}\right)_{\mathfrak{n}}\right) \otimes_{C} \mathfrak{n}^{*}
$$

remains surjective when passing to the kernel of $h+1$; that is, there is an exact sequence

$$
0 \rightarrow H^{1}\left(\mathscr{F} \ell,\left(\mathcal{F}_{2}\right)^{\mathfrak{n}}\right)_{\mu} \rightarrow H^{1}\left(\mathscr{F} \ell, \mathcal{F}_{2}\right)_{\mu}^{\mathfrak{n}} \rightarrow\left(H^{0}\left(\mathscr{F} \ell,\left(\mathcal{F}_{2}\right)_{\mathfrak{n}}\right) \otimes_{C} \mathfrak{n}^{*}\right)_{\mu} \rightarrow 0
$$

In fact, this is really a formal consequence of the previous lemma. Consider the following commutative diagram:

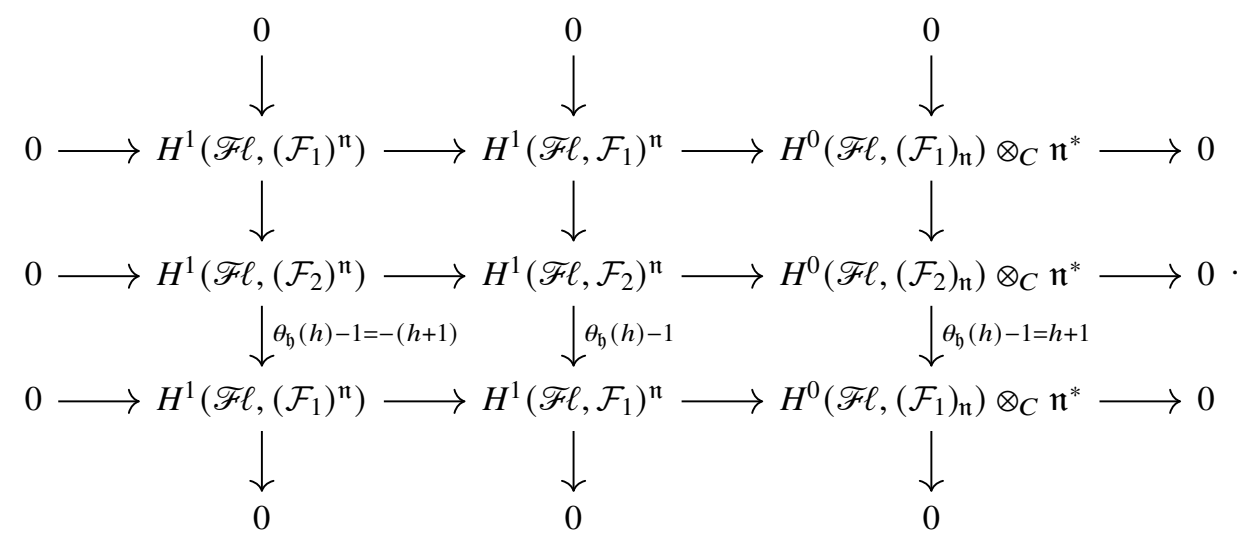

Let $f \in H^{0}\left(\mathscr{F} \ell,\left(\mathcal{F}_{1}\right)_{\mathfrak{n}}\right) \otimes_{C} \mathfrak{n}^{*} \subset H^{0}\left(\mathscr{F} \ell,\left(\mathcal{F}_{2}\right)_{\mathfrak{n}}\right) \otimes_{C} \mathfrak{n}^{*}$. We can find a preimage $\tilde{f} \in H^{1}\left(\mathscr{F} \ell, \mathcal{F}_{1}\right)^{\mathfrak{n}}$. Then $(h+1) \cdot \tilde{f} \in H^{1}\left(\mathscr{F} \ell,\left(\mathcal{F}_{1}\right)^{\mathfrak{n}}\right)$. By Lemma 5.4.4, there exists $\tilde{g} \in H^{1}\left(\mathscr{F} \ell,\left(\mathcal{F}_{2}\right)^{\mathfrak{n}}\right)$ such that $(h+1) \cdot \tilde{g}=(h+1) \cdot \tilde{f}$. Now $g:=\tilde{f}-\tilde{g}$ defines an element in $H^{1}\left(\mathscr{F} \ell, \mathcal{F}_{2}\right)^{\mathfrak{n}}$ lifting $f$ and annihilated by $h+1$.

Since $H^{1}\left(\mathscr{F} \ell, \mathcal{F}_{1}\right)^{\mathfrak{n}}$ is computed in Section 5.3.17, we obtain the following result. The last part is a restatement of Theorem 5.3.18.

Theorem 5.4.6. Suppose $\mu=\left(k_{1}, k_{1}+1\right)$; that is, $\mu(h)=-1$. There exists an exact sequence

$$
0 \rightarrow N_{1} \cdot e_{1}^{-1} t^{k_{1}+1} \rightarrow \tilde{H}^{1}\left(K^{p}, C\right)_{\mu}^{\mathrm{la}} \rightarrow M_{1}^{\dagger}\left(K^{p}\right) \cdot e_{1}^{-1} t^{k_{1}+1} \rightarrow 0
$$

where $N_{1}$ sits inside the exact sequence

$$
0 \rightarrow M_{1}^{\dagger}\left(K^{p}\right) / M_{1}\left(K^{p}\right) \rightarrow N_{1} \rightarrow \underset{K_{p}}{\lim } H^{1}\left(\mathcal{X}_{K^{p}} K_{p}, \omega^{1}\right) \rightarrow 0 .
$$

Moreover, $\tilde{H}^{1}\left(K^{p}, C\right)_{\mu}^{\mathrm{la},\left(k_{1}+1, k_{1}\right)} \subset \tilde{H}^{1}\left(K^{p}, C\right)_{\mu}^{\mathrm{la}}$ is identified with the pullback of $M_{1}\left(K^{p}\right) \cdot e_{1}^{-1} t^{k_{1}+1} \subset$ $M_{1}^{\dagger}\left(K^{p}\right) \cdot e_{1}^{-1} t^{k_{1}+1}$; that is, there is an exact sequence

$$
0 \rightarrow N_{1} \cdot e_{1}^{-1} t^{k_{1}+1} \rightarrow \tilde{H}^{1}\left(K^{p}, C\right)_{\mu}^{\mathrm{la},\left(k_{1}+1, k_{1}\right)} \rightarrow M_{1}\left(K^{p}\right) \cdot e_{1}^{-1} t^{k_{1}+1} \rightarrow 0 .
$$

All of the maps are Hecke and $B \times G_{\mathbb{Q}_{p}}$-equivariant. 


\section{Applications}

In this section, we present several applications of the main results of previous section to the study of overconvergent modular forms and the Fontaine-Mazur conjecture in the irregular case. One main ingredient is Emerton's local-global compatibility result, which allows us to study completed cohomology using the ( $p$-adic) representation theory of $\mathrm{GL}_{2}\left(\mathbb{Q}_{p}\right)$.

\subsection{Hecke algebra}

6.1.1. First we recall the definition of the (big) Hecke algebra associated to completed cohomology. Let $K^{p}=\prod_{l \neq p} K_{l} \subset \mathrm{GL}_{2}\left(\mathbb{A}_{f}^{p}\right)$ be a tame level and $S$ a finite set of rational primes containing $p$ and all places $l$ for which $K_{l}$ is not maximal. As usual, we denote the double coset action of

$$
\left[K_{l}\left(\begin{array}{ll}
l & 0 \\
0 & 1
\end{array}\right) K_{l}\right]
$$

by $T_{l}$ and the double coset action of

$$
\left[K_{l}\left(\begin{array}{ll}
l & 0 \\
0 & l
\end{array}\right) K_{l}\right]
$$

by $S_{l}$. For any open compact subgroup $K_{p}$ of $\mathrm{GL}_{2}\left(\mathbb{Q}_{p}\right)$, we define

$$
\mathbb{T}\left(K^{p} K_{p}\right) \subset \operatorname{End}_{\mathbb{Z}_{p}}\left(H_{\text {ét }}^{1}\left(\mathcal{Y}_{K^{p} K_{p}}, \mathbb{Z}_{p}\right)\right)
$$

as the $\mathbb{Z}_{p}$-subalgebra generated by Hecke operators $T_{l}, S_{l}^{ \pm 1}$ at places $l \notin S$. As the notation suggests, this does not depend on the choice of $S$ because of the existence of continuous 2-dimensional determinants of $G_{\mathbb{Q}, S}$ over $\mathbb{T}\left(K^{p} K_{p}\right)$ and Chebatorev's density theorem. Now we define the Hecke algebra of tame level $K^{p}$ as

$$
\mathbb{T}\left(K^{p}\right):=\lim _{K_{p} \subset \mathrm{GL}_{2}\left(\mathbb{Q}_{p}\right)} \mathbb{T}\left(K^{p} K_{p}\right)
$$

This is a complete semi-local $\mathbb{Z}_{p}$-algebra and $\mathbb{T}\left(K^{p}\right) / \mathrm{m}$ is a finite field for any maximal ideal $\mathrm{m}$. It acts faithfully on $\tilde{H}^{1}\left(K^{p}, \mathbb{Z}_{p}\right)$ and commutes with the action of $\mathrm{GL}_{2}\left(\mathbb{Q}_{p}\right) \times G_{\mathbb{Q}_{p}}$. Moreover, there is a continuous 2-dimensional determinant $D_{S}$ of $G_{\mathbb{Q}, S}$ valued in $\mathbb{T}\left(K^{p}\right)$ in the sense of Chenevier [Che14] satisfying the following property ${ }^{5}$ : for any $l \notin S$, the characteristic polynomial of $D_{S}\left(\mathrm{Frob}_{l}\right)$ is

$$
X^{2}-l^{-1} T_{l} X+l^{-1} S_{l}
$$

Note that its twist by inverse of the cyclotomic character is also the Eichler-Shimura congruence relation ([Del71, Théorème 4.9]); that is,

$$
\operatorname{Frob}_{l}^{2}-T_{l} \text { Frob }_{l}+l S_{l}=0
$$

on $\tilde{H}^{1}\left(K^{p}, \mathbb{Z}_{p}\right)$. This can be checked first on finite levels and then by passing to the limit over $K_{p}$. Note that $T_{l}, S_{l}^{ \pm 1}, l \notin S$ generate $\mathbb{T}\left(K^{p}\right)$ topologically.

Let $\lambda: \mathbb{T}\left(K^{p}\right) \rightarrow \overline{\mathbb{Q}}_{p}$ be a $\mathbb{Z}_{p}$-algebra homomorphism. We can associate an odd semi-simple Galois representation (unique up to conjugation)

$$
\rho_{\lambda}: G_{\mathbb{Q}, S} \rightarrow \mathrm{GL}_{2}\left(\overline{\mathbb{Q}}_{p}\right)
$$

\footnotetext{
${ }^{5}$ The existence of $D_{S}$ implies that $\mathbb{T}\left(K^{p}\right)$ is Noetherian. Note that $T_{l}, S_{l}^{ \pm 1}, l \notin S$ generate $\mathbb{T}\left(K^{p}\right)$ topologically; hence, $\mathbb{T}\left(K^{p}\right)$ receives a surjective map from a finite product of some universal deformation rings of 2-dimensional determinants of $G_{\mathbb{Q}, S}$, which are Noetherian by the work of Chenevier.
} 
whose determinant is $\lambda \circ D_{S}$. Here odd means $\operatorname{det}\left(\rho_{\lambda}(c)\right)=-1$ for any complex conjugation $c \in G_{\mathbb{Q}, S}$. Let $\tilde{H}^{1}\left(K^{p}, \overline{\mathbb{Q}}_{p}\right)=\tilde{H}^{1}\left(K^{p}, \mathbb{Q}_{p}\right) \otimes \overline{\mathbb{Q}}_{p}$ and let $\mathfrak{p}_{\lambda}:=\operatorname{ker}\left(\lambda \otimes \overline{\mathbb{Q}}_{p}\right) \in \operatorname{Spec} \mathbb{T}\left(K^{p}\right) \otimes_{\mathbb{Q}_{p}} \overline{\mathbb{Q}}_{p}$. If $\rho_{\lambda}$ is irreducible, by the Eichler-Shimura relation, we have

$$
\operatorname{Hom}_{G_{\mathbb{Q}}}\left(\rho_{\lambda}(-1), \tilde{H}^{1}\left(K^{p}, \overline{\mathbb{Q}}_{p}\right)\right)=\operatorname{Hom}_{G_{\mathbb{Q}}}\left(\rho_{\lambda}(-1), \tilde{H}^{1}\left(K^{p}, \overline{\mathbb{Q}}_{p}\right)\left[\mathfrak{p}_{\lambda}\right]\right),
$$

where $\rho_{\underline{\lambda}}(-1)=\rho_{\lambda} \otimes \varepsilon^{-1}$ is the twist of $\rho_{\lambda}$ by inverse of the $p$-adic cyclotomic character and $\tilde{H}^{1}\left(K^{p}, \overline{\mathbb{Q}}_{p}\right)\left[\mathfrak{p}_{\lambda}\right]$ denotes the $\lambda$-isotypic subspace. By the main result of [BLR91], $\tilde{H}^{1}\left(K^{p}, \overline{\mathbb{Q}}_{p}\right)\left[\mathfrak{p}_{\lambda}\right]$ is $\rho_{\lambda}(-1)$-isotypic in the sense that

$$
\tilde{H}^{1}\left(K^{p}, \overline{\mathbb{Q}}_{p}\right)\left[\mathfrak{p}_{\lambda}\right]=\rho_{\lambda}(-1) \otimes_{\overline{\mathbb{Q}}_{p}} \operatorname{Hom}_{G_{\mathbb{Q}}}\left(\rho_{\lambda}(-1), \tilde{H}^{1}\left(K^{p}, \overline{\mathbb{Q}}_{p}\right)\right) .
$$

We remark that the centre $\mathbb{A}_{f}^{\times}$of $\mathrm{GL}_{2}\left(\mathbb{A}_{f}\right)$ acts via $\operatorname{det}\left(\rho_{\lambda}\right) \varepsilon^{-1}$ on $\tilde{H}^{1}\left(K^{p}, \overline{\mathbb{Q}}_{p}\right)\left[\mathfrak{p}_{\lambda}\right]$ via global class field theory.

Definition 6.1.2. Let

$$
\rho: G_{\mathbb{Q}} \rightarrow \mathrm{GL}_{2}\left(\overline{\mathbb{Q}}_{p}\right)
$$

be a continuous 2-dimensional $p$-adic Galois representation. We say $\rho$ is

$\circ$ pro-modular if there exists a tame level $K^{p}$ and $\lambda: \mathbb{T}\left(K^{p}\right) \rightarrow \overline{\mathbb{Q}}_{p}$ such that $\rho \cong \rho_{\lambda}$;

○ pro-cohomological if $\operatorname{Hom}_{G_{\mathbb{Q}}}\left(\rho(-1), \tilde{H}^{1}\left(K^{p}, \overline{\mathbb{Q}}_{p}\right)\right) \neq 0$ for some tame level $K^{p}$.

Clearly, $\rho$ is pro-modular if it is pro-cohomological and irreducible by our previous discussion. Conversely, we have the following result.

Lemma 6.1.3. Let $\rho=\rho_{\lambda}$ be an irreducible pro-modular representation for some $K^{p}$ and $\lambda$. The following statements are equivalent:

(1) $\rho$ is pro-cohomological.

(2) $\tilde{H}^{1}\left(K^{p}, \overline{\mathbb{Q}}_{p}\right)\left[\mathfrak{p}_{\lambda}\right] \neq 0$.

(3) $\tilde{H}^{1}\left(K^{p}, C\right)\left[\mathfrak{p}_{\lambda}\right] \neq 0$.

Proof. The first two are equivalent by the Eichler-Shimura relation. The equivalence between the last two is a consequence of the following lemma.

Lemma 6.1.4. Let $\rho=\rho_{\lambda}$ be a pro-modular representation for some $K^{p}$ and $\lambda$. Then

$$
\tilde{H}^{1}\left(K^{p}, \overline{\mathbb{Q}}_{p}\right)\left[\mathfrak{p}_{\lambda}\right] \widehat{\otimes}_{\overline{\mathbb{Q}}_{p}} C \cong \tilde{H}^{1}\left(K^{p}, C\right)\left[\mathfrak{p}_{\lambda}\right],
$$

where $\tilde{H}^{1}\left(K^{p}, \overline{\mathbb{Q}}_{p}\right)\left[\mathfrak{p}_{\lambda}\right]$ is endowed with a norm with unit ball $\left(\tilde{H}^{1}\left(K^{p}, \mathbb{Z}_{p}\right) \otimes \overline{\mathbb{Z}}_{p}\right)\left[\mathfrak{p}_{\lambda}\right]$.

Proof. Choose generators $g_{1}, \cdots, g_{s}$ of $\mathfrak{p}_{\lambda}$ as a $\mathbb{T}\left(K^{p}\right) \otimes \overline{\mathbb{Q}}_{p}$-module. We have an exact sequence

$$
0 \rightarrow \tilde{H}^{1}\left(K^{p}, \overline{\mathbb{Q}}_{p}\right)\left[\mathfrak{p}_{\lambda}\right] \rightarrow \tilde{H}^{1}\left(K^{p}, \overline{\mathbb{Q}}_{p}\right) \stackrel{\left(g_{1}, \cdots, g_{s}\right)}{\longrightarrow} \bigoplus_{i=1}^{s} \tilde{H}^{1}\left(K^{p}, \overline{\mathbb{Q}}_{p}\right)
$$

which is continuous with respect to the $p$-adic topology; that is, defined by the unit open ball $\tilde{H}^{1}\left(K^{p}, \mathbb{Z}_{p}\right) \otimes \overline{\mathbb{Z}}_{p}$. Note that the second map is strict. This sequence remains exact after taking $p$-adic completion, which is exactly what we want.

We conclude this subsection by the following result on infinitesimal characters.

Proposition 6.1.5. Suppose $\rho=\rho_{\lambda}$ is pro-cohomological and irreducible and has Hodge-Tate-Sen weights $(a, b)$. Then as a representation of $\mathrm{GL}_{2}\left(\mathbb{Q}_{p}\right)$, the locally analytic vectors $\tilde{H}^{1}\left(K^{p}, C\right)^{\mathrm{la}}\left[\mathfrak{p}_{\lambda}\right] \neq 0$ and have infinitesimal character $\{(-a-1,-b),(-b-1,-a)\} \subset \mathfrak{h}^{*}$. 
Proof. Note that $\rho$ can be defined over a finite extension $E$ of $\mathbb{Q}_{p}$. Hence, $\tilde{H}^{1}\left(K^{p}, \overline{\mathbb{Q}}_{p}\right)\left[\mathfrak{p}_{\lambda}\right] \cap$ $\tilde{H}^{1}\left(K^{p}, \mathbb{Q}_{p}\right) \otimes E \neq 0$ and is an admissible representation of $\mathrm{GL}_{2}\left(\mathbb{Q}_{p}\right)$. It has nonzero locally analytic vectors by the main result of [ST03]. Since det $\rho$ has Hodge-Tate-Sen weight $a+b$, the centre $C z$ of $\mathfrak{g}$ acts on $\tilde{H}^{1}\left(K^{p}, C\right)^{\text {la }}\left[\mathfrak{p}_{\lambda}\right]$ via $z \mapsto-(a+b)-1$. Now the claim for infinitesimal character follows directly from Corollary 4.2.8 and Theorem 5.1.11.

Remark 6.1.6. A higher dimensional generalisation of this result was obtained by DospinescuPaškūnas-Schraen in [DPS20, Theorem 1.4].

\subsection{A classicality result for overconvergent weight 1 forms}

6.2.1. Suppose $\rho=\rho_{\lambda}$ has equal Hodge-Tate-Sen weights 0,0 . (It is easy to reduce to this case by twisting by a character if weights are $a, a$.) There are two possibilities for $\rho$ : either $\left.\rho\right|_{G_{Q_{p}}}$ is Hodge-Tate, or not. In other words, $\left(\rho \otimes_{\mathbb{Q}_{p}} C\right)^{G_{Q_{p}}}$ is a $\overline{\mathbb{Q}}_{p}$-vector space of dimension 2 or 1 . Recall that by DeligneSerre [DS74], the 2-dimensional Galois representation associated to a classical weight 1 eigenform is Hodge-Tate at $p$. The main result of this subsection gives a converse.

Recall that there is a natural action of $B$ on $M_{1}^{\dagger}\left(K^{p}\right)$. Let $N_{0} \subset B$ be $\left(\begin{array}{cc}1 & \mathbb{Z}_{p} \\ 0 & 1\end{array}\right)$. Then we have the usual operator

$$
U_{p}:=\sum_{i=0}^{p-1}\left(\begin{array}{ll}
p & i \\
0 & 1
\end{array}\right)
$$

acting on $M_{1}^{\dagger}\left(K^{p}\right)^{N_{0}}$. Note that Theorem 5.4.2 implies that Hecke algebra $\mathbb{T}\left(K^{p}\right)$ also acts on the space of overconvergent modular forms.

Theorem 6.2.2. Suppose $\rho=\rho_{\lambda}$ is pro-modular for some tame level $K^{p}$. If $M_{1}^{\dagger}\left(K^{p}\right)^{N_{0}}\left[\mathfrak{p}_{\lambda}\right]$ has a nonzero $U_{p}$-eigenvector and $\left.\rho\right|_{G_{Q_{p}}}$ is Hodge-Tate of weights 0,0 , then $\rho$ comes from a classical weight 1 eigenform; that is, $M_{1}\left(K^{p}\right)\left[\mathfrak{p}_{\lambda}\right] \neq 0$.

Remark 6.2.3. Theorem 6.2.2 implies that an overconvergent weight 1 modular form of finite slope is classical if its associated Galois representation is Hodge-Tate. In particular, this gives a different proof of the main result of Buzzard-Taylor [BT99] in the ordinary case.

Note that we do not assume the eigenvalue of $U_{p}$ is nonzero. In fact, using Colmez's Kirillov model, we will see that the kernel of $U_{p}$ is always nonzero if we know the local-global compatibility at $p$ (in the sense of Emerton).

6.2.4. The rest of this subsection is devoted to the proof of Theorem 6.2.2. As mentioned in the Introduction, we will need Hecke operators at bad places in the proof. After making a right translation by some element in $\mathrm{GL}_{2}\left(\mathbb{A}_{f}^{p}\right)$ and shrinking the level, we can find a finite set of rational primes $S$ containing $p$ and an integer $m>0$ and assume the following: $K^{p}=\prod_{l \neq p} K_{l} \subset \prod_{l \neq p} \mathrm{GL}_{2}\left(\mathbb{Z}_{l}\right)$, where $K_{l}=\mathrm{GL}_{2}\left(\mathbb{Z}_{l}\right)$ for $l \notin S$, and

$$
K_{l}=\Gamma_{1}\left(l^{m}\right)=\left\{\left(\begin{array}{ll}
a & b \\
c & d
\end{array}\right), a-1, c, d-1 \in l^{m} \mathbb{Z}_{l}\right\}, l \in S \backslash\{p\} .
$$

Moreover, $M_{1}^{\dagger}\left(K^{p} \Gamma_{1}\left(p^{m}\right)\right)\left[\mathfrak{p}_{\lambda}\right]$ has a nonzero eigenvector of $U_{p}$. See Section 5.2.4 for the notation here. Let $K_{p}=\Gamma_{1}\left(p^{n}\right)$ for some $n \geq m$ and $K=K^{p} K_{p}$. Let $A_{S}=\prod_{l \in S} \mathbb{Z}_{l}^{\times} \subset \prod_{l \in S} \mathrm{GL}_{2}\left(\mathbb{Z}_{l}\right)$ be the subgroup of the form $\left(\begin{array}{ll}* & 0 \\ 0 & 1\end{array}\right)$. Then $A_{S}$ is in the normaliser of $\prod_{l \in S} K_{l}$; hence, $A_{S}$ acts on $\mathcal{X}_{K}$ by right translation, which induces an action of $A_{S}$ on $M_{1}^{\dagger}(K)$. Clearly, this action factors through a finite group 
and we can find a (finite-order) character $\psi: A_{S} \rightarrow \overline{\mathbb{Q}}_{p}^{\times}$such that the $\psi$-isotypic component

$$
M_{1}^{\dagger}(K)^{\psi}\left[\mathfrak{p}_{\lambda}\right]
$$

contains a nonzero eigenvector of $U_{p}$. For $l \in S$, we denote by $U_{l}$ the abstract double coset action of

$$
\left[K_{l}\left(\begin{array}{ll}
l & 0 \\
0 & 1
\end{array}\right) K_{l}\right] \text {. }
$$

These operators act naturally on $M_{1}^{\dagger}(K)$. If, moreover, $l \neq p$, then $U_{l}$ acts naturally on $\tilde{H}^{1}\left(K^{p}, C\right)$. We denote by

$$
\tilde{\mathbb{T}}\left(K^{p}\right) \subset \operatorname{End}\left(\tilde{H}^{1}\left(K^{p}, C\right)\right)
$$

the $\mathbb{T}\left(K^{p}\right)$-subalgebra generated by $U_{l}, l \in S \backslash\{p\}$.

Lemma 6.2.5. $\tilde{\mathbb{T}}\left(K^{p}\right)$ is a finite $\mathbb{T}\left(K^{p}\right)$-module; that is, each $U_{l}$ is integral over $\mathbb{T}\left(K^{p}\right), l \in S \backslash\{p\}$.

Proof. Fix $l \in S \backslash\{p\}$. Since $\lim _{\longrightarrow} K_{p}^{\prime} H_{\text {êt }}^{1}\left(\mathcal{X}_{K^{p} K_{p}^{\prime}}, \mathbb{Q}_{p}\right)$ is dense in $\tilde{H}^{1}\left(K^{p}, \mathbb{Q}_{p}\right)$ (Theorem 2.2.16 (iv) of [Eme06b]), it suffices to find a monic polynomial $P_{l}(X) \in \mathbb{T}\left(K^{p}\right)[X]$ such that $P_{l}\left(U_{l}\right)$ acts as zero on $H_{\text {ét }}^{1}\left(\mathcal{X}_{K^{p} K_{p}^{\prime}}, \mathbb{Q}_{p}\right)$ for any open subgroup $K_{p}^{\prime}$ of $\mathrm{GL}_{2}\left(\mathbb{Q}_{p}\right)$. Fix a lift of geometric Frobenius Frob $\in_{l} G_{\mathbb{Q}_{l}}$ whose image in $G_{\mathbb{Q}_{l}}^{\text {ab }}$ corresponds to $l \in \mathbb{Q}_{l}^{\times}$via the local Artin map. Recall that there is a determinant $D_{S}$ of $G_{\mathbb{Q}, S}$ valued in $\mathbb{T}\left(K^{p}\right)$. We denote by $Q_{l}(X) \in \mathbb{T}\left(K^{p}\right)[X]$ the characteristic polynomial of $l \mathrm{Frob}_{l} \in \mathbb{T}\left(K^{p}\right)\left[G_{\mathbb{Q}, S}\right]$. We claim that

$$
P_{l}(X):=X^{m+1} Q_{l}(X)
$$

works. To see this, since $H_{\text {et }}^{1}\left(\mathcal{X}_{K^{p}} K_{p}^{\prime}, \overline{\mathbb{Q}}_{p}\right)$ can be decomposed as a direct sum of $\left(\pi_{f}\right)^{K^{p} K_{p}^{\prime}}$ for some cuspidal automorphic representations $\pi=\pi_{f} \otimes \pi_{\infty}$ on $\mathrm{GL}_{2}(\mathbb{A})$, it is enough to show $P_{l}\left(U_{l}\right)=0$ on each nonzero $\left(\pi_{f}\right)^{K^{p}} K_{p}^{\prime}$. By the theory of newforms, $\left(\pi_{l}\right)^{K_{l}}$ has dimension at most $m+1$ and $U_{l}$ is not nilpotent only when $\pi_{l}$ is special or a principal series. Moreover, $U_{l}$ acts semi-simply on generalised eigenspaces associated to nonzero eigenvalues. See, for example, Corollary 2.2 of [Hid89]. The localglobal compatibility then implies that a nonzero eigenvalue of $U_{l}$ must be a root of $Q_{l}(X)$. Hence, $P_{l}\left(U_{l}\right)=0$ on $\left(\pi_{f}\right)^{K^{p} K_{p}^{\prime}}$.

Now we consider the action of $\tilde{\mathbb{T}}\left(K^{p}\right)\left[U_{p}\right]$ on $M_{1}^{\dagger}(K)^{\psi}$.

Lemma 6.2.6. $\lambda: \mathbb{T}\left(K^{p}\right) \rightarrow \overline{\mathbb{Q}}_{p}$ can be extended to $\lambda^{\prime}: \tilde{\mathbb{T}}\left(K^{p}\right)\left[U_{p}\right] \rightarrow C$ such that

$$
M_{1}^{\dagger}\left(K^{p} \Gamma_{1}\left(p^{n}\right)\right)^{\psi}\left[\mathfrak{p}_{\lambda^{\prime}}\right] \neq 0,
$$

where $\mathfrak{p}_{\lambda^{\prime}}$ denotes the kernel of $\lambda^{\prime}$. Moreover, for any such $\lambda^{\prime}$, we have

$$
\operatorname{dim}_{C} M_{1}^{\dagger}\left(K^{p} \Gamma_{1}\left(p^{n}\right)\right)^{\psi}\left[\mathfrak{p}_{\lambda^{\prime}}\right]=1 .
$$

In particular, we obtain the following after taking direct limit over all n:

$$
\operatorname{dim}_{C} \underset{n}{\lim _{n}} M_{1}^{\dagger}\left(K^{p} \Gamma_{1}\left(p^{n}\right)\right)^{\psi}\left[\mathfrak{p}_{\lambda^{\prime}}\right]=\operatorname{dim}_{C} M_{1}^{\dagger}\left(K^{p}\right)^{\psi, N_{0}}\left[\mathfrak{p}_{\lambda^{\prime}}\right]=1
$$

Proof. Let $\alpha \in C$ be an eigenvalue of $U_{p}$ on $M_{1}^{\dagger}(K)^{\psi}\left[\mathfrak{p}_{\lambda}\right]$. Then $\tilde{\mathbb{T}}\left(K^{p}\right) /\left(\mathfrak{p}_{\lambda}\right)$ acts on the $\alpha$-eigenspace of $M_{1}^{\dagger}(K)^{\psi}\left[\mathfrak{p}_{\lambda}\right]$. Since $\tilde{\mathbb{T}}\left(K^{p}\right) /\left(\mathfrak{p}_{\lambda}\right)$ is Artinian by our previous lemma, we can find $\lambda^{\prime}: \tilde{\mathbb{T}}\left(K^{p}\right)\left[U_{p}\right] \rightarrow C$ which sends $U_{p}$ to $\alpha$ and extends $\lambda$ and $M_{1}^{\dagger}(K)^{\psi}\left[\mathfrak{p}_{\lambda^{\prime}}\right] \neq 0$. To see the multiplicity 1 claim, we remark that $A_{S}$ acts transitively on all connected components of $\mathcal{X}_{K}$. Hence, any element of $M_{1}^{\dagger}(K)^{\psi}$ 
is determined by its value on one component. Since elements in $M_{1}^{\dagger}(K)^{\psi}\left[\mathfrak{p}_{\lambda^{\prime}}\right]$ are eigenvectors of $T_{l}, S_{l}, l \notin S$ and $U_{l}, l \in S$, we conclude from usual $q$-expansion principle that this space has dimension 1.

Proof of Theorem 6.2.2. If $\rho$ is reducible, then it is a sum of two finite-order characters. The theory of Eisenstein series shows that it comes from a classical modular form. Hence, we may assume $\rho$ is irreducible.

Assume $\rho$ is not classical; that is, $M^{1}\left(K^{p}\right)\left[\mathfrak{p}_{\lambda}\right]=0$. Since $M^{1}\left(K^{p}\right)=\lim _{\longrightarrow} K_{p}^{\prime} H^{0}\left(\mathcal{X}_{K^{p}} K_{p}^{\prime}, \omega\right)$ is a direct sum of $\left(\pi_{f}\right)^{K^{p}}$ for some automorphic representations $\pi$ of $\mathrm{GL}_{2}(\mathbb{A})$, the localisation

$$
M^{1}\left(K^{p}\right)_{\mathfrak{p}_{\lambda}}=0
$$

Similarly, by Serre duality and the Kodaira-Spencer isomorphism, we also have

$$
\left.\underset{K_{p}^{\prime}}{\left(\lim ^{\prime}\right.} H^{1}\left(\mathcal{X}_{K^{p} K_{p}^{\prime}}, \omega^{1}\right)\right)_{\mathfrak{p}_{\lambda}}=0 .
$$

Hence, by the second part of Theorem 5.4.6, $\left(\tilde{H}^{1}\left(K^{p}, C\right)_{(0,1)}^{\mathrm{la},(1,0)}\right)_{\mathfrak{p}_{\lambda \cdot t}}=M_{1}^{\dagger}\left(K^{p}\right)_{\mathfrak{p}_{\lambda}} \cdot e_{1}^{-1} t$ and therefore

$$
\tilde{H}^{1}\left(K^{p}, C\right)_{(0,1)}^{\mathrm{la},(1,0)}\left[\mathfrak{p}_{\lambda \cdot t}\right]=M_{1}^{\dagger}\left(K^{p}\right)\left[\mathfrak{p}_{\lambda}\right] \cdot e_{1}^{-1} t,
$$

where $\lambda \cdot t: \mathbb{T}\left(K^{p}\right) \rightarrow \overline{\mathbb{Q}}_{p}$ denotes 'the twist of $\lambda$ by $t^{\prime}$; that is, sends $T_{l}$ to $\lambda\left(T_{l}\right) l^{-1}$. It can be checked that $\rho_{\lambda \cdot t}(-1)=\rho_{\lambda}$. Hence, $\tilde{H}^{1}\left(K^{p}, \overline{\mathbb{Q}}_{p}\right)\left[\mathfrak{p}_{\lambda \cdot t}\right]$ is $\rho$-isotypic. On the other hand, by our assumption that $\rho$ is Hodge-Tate and Theorem 5.1.11, we have

$$
\tilde{H}^{1}\left(K^{p}, C\right)_{(0,1)}^{\mathrm{la}}\left[\mathfrak{p}_{\lambda \cdot t}\right]=\tilde{H}^{1}\left(K^{p}, C\right)_{(0,1)}^{\mathrm{la},(1,0)}\left[\mathfrak{p}_{\lambda \cdot t}\right] .
$$

If we write $W=\operatorname{Hom}_{G_{\mathbb{Q}}}\left(\rho, \tilde{H}^{1}\left(K^{p}, \overline{\mathbb{Q}}_{p}\right)\right)$, then by Lemma 6.1.4 and our discussion in Section 6.1.1,

$$
\tilde{H}^{1}\left(K^{p}, C\right)_{(0,1)}^{\mathrm{la}}\left[\mathfrak{p}_{\lambda \cdot t}\right]=\rho \otimes_{\overline{\mathbb{Q}}_{p}}\left(W \widehat{\otimes}_{\overline{\mathbb{Q}}_{p}} C\right)_{(0,1)}^{\mathrm{la}},
$$

where $W$ is endowed a norm with unit ball $\operatorname{Hom}_{G_{\mathbb{Q}}}\left(\rho^{o}, \tilde{H}^{1}\left(K^{p}, \mathbb{Z}_{p}\right) \otimes \overline{\mathbb{Z}}_{p}\right)$ and $\rho^{o} \subset \rho$ is any $G_{\mathbb{Q}^{-s t a b l e}}$ lattice. Note that $A_{S} \times \tilde{\mathbb{T}}\left(K^{p}\right)$ acts naturally on $W$ and $U_{p}$ acts on its $N_{0}$-fixed vectors. Fix $\lambda^{\prime}$ as in Lemma 6.2.6 and consider its 'twist by $e_{1}^{-1} t$ '

$$
\lambda^{\prime \prime}: \tilde{T}\left(K^{p}\right)\left[U^{p}\right] \rightarrow C
$$

which sends $T_{l}$ to $\lambda^{\prime}\left(T_{l}\right) l^{-1}$ for $l \notin S$ and sends $U_{l}$ to $\lambda^{\prime}\left(U_{l}\right) l^{-1}$ for $l \in S$. Then we have

$$
M_{1}^{\dagger}\left(K^{p}\right)^{\psi, N_{0}}\left[\mathfrak{p}_{\lambda^{\prime}}\right] \cdot e_{1}^{-1} t=\tilde{H}^{1}\left(K^{p}, C\right)_{(0,1)}^{\mathrm{la}, \psi, N_{0}}\left[\mathfrak{p}_{\lambda^{\prime \prime}}\right]=\rho \otimes_{\overline{\mathbb{Q}}_{p}}\left(W \widehat{\otimes}_{\overline{\mathbb{Q}}_{p}} C\right)_{(0,1)}^{\mathrm{la}, \psi, N_{0}}\left[\mathfrak{p}_{\lambda^{\prime \prime}}\right] .
$$

The left-hand side has dimension 1 over $C$ by Lemma 6.2.6. However, the last term in the equality has dimension at least 2 because $\rho$ is 2 -dimensional! Thus, we get a contradiction. This proves that $\rho$ has to be classical.

\subsection{Local-global compatibility}

6.3.1. In order to better understand $\tilde{H}^{1}\left(K^{p}, \overline{\mathbb{Q}}_{p}\right)\left[\mathfrak{p}_{\lambda}\right]$ for a pro-modular $\lambda$, we need Emerton's localglobal compatibility conjecture, which gives a description of $\tilde{H}^{1}\left(K^{p}, \overline{\mathbb{Q}}_{p}\right)\left[\mathfrak{p}_{\lambda}\right]$ in terms of $p$-adic local Langlands for $\mathrm{GL}_{2}\left(\mathbb{Q}_{p}\right)$ as established by Breuil, Colmez, Berger, Kisin, Paškūnas. In [Eme11, Eme06a], Emerton formulated and proved his conjecture assuming the residual representation is irreducible and 
generic. Building upon the work of Paškūnas, we gave a proof (which is complete when $p \geq 5$ ) in [Pan19] in the case of definite quaternion algebras. We will mostly follow the argument in Section 3 of [Pan19] and also Section 5 of [Pas18]. One key step is to prove the density of algebraic vectors when we are localising at an Eisenstein maximal ideal. This part might be of some independent interest.

6.3.2. We begin by recalling work of Paškūnas on representations of $\mathrm{GL}_{2}\left(\mathbb{Q}_{p}\right)$. See [Paš13] and also the introduction of [Paš16]. Fix $E$ a finite extension of $\mathbb{Q}_{p}$ with ring of integers $\mathcal{O}$, residue field $\mathbb{F}$, and fix a uniformiser $\varpi$. Write $G=\mathrm{GL}_{2}\left(\mathbb{Q}_{p}\right)$ and let $\zeta: \mathbb{Q}_{p}^{\times} \rightarrow \mathcal{O}^{\times}$be a continuous character. Following Paškūnas, we denote by $\operatorname{Mod}_{G, \zeta}^{1}(\mathcal{O d m})$ the category of smooth, locally admissible $G$-representations on $\mathcal{O}$-torsion modules with central character $\zeta$.

Let $\operatorname{Irr}_{G}^{\mathrm{adm}}$ be the set of $G$-irreducible representations in $\operatorname{Mod}_{G, \zeta}^{\mathrm{ladm}}(\mathcal{O})$. There is a natural equivalence relation $\sim$ on $\operatorname{Irr}_{G}^{\mathrm{adm}}$ defined as follows: $\pi \sim \tau$ if $\pi=\tau$ or there exists a sequence $\pi_{0}=\pi, \cdots, \pi_{n}=\tau \in$ $\operatorname{Irr}_{G}^{\text {adm }}$ such that $\operatorname{Ext}^{1}\left(\pi_{i}, \pi_{i+1}\right) \neq 0$ or $\operatorname{Ext}^{1}\left(\pi_{i+1}, \pi_{i}\right) \neq 0$. An equivalence class $\mathfrak{B} \in \operatorname{Irr}_{G}^{\text {adm }} / \sim$ is called a block of $\operatorname{Mod}_{G, \zeta}^{1 \mathrm{adm}}(\mathcal{O})$. There is a natural decomposition

$$
\operatorname{Mod}_{G, \zeta}^{\operatorname{ladm}}(\mathcal{O}) \cong \prod_{\mathfrak{B} \in \operatorname{Irr}_{G}^{\mathrm{adm}} / \sim} \operatorname{Mod}_{G, \zeta}^{\mathrm{ladm}}(\mathcal{O})[\mathfrak{B}]
$$

where $\operatorname{Mod}_{G, \zeta}^{1 \mathrm{adm}}(\mathcal{O})[\mathfrak{B}]$ denotes the full subcategory of representations with irreducible constituents in $\mathfrak{B}$. A complete list of blocks containing an absolutely irreducible representation can be found on the beginning of p. 3 of [Paš16].

The semi-simple mod $p$ correspondence gives a bijection between isomorphism classes of 2dimensional absolutely semi-simple $\mathbb{F}$-representations $\bar{\rho}_{\mathfrak{B}}$ of $G_{\mathbb{Q}_{p}}$ and blocks containing an absolutely irreducible representation. It should be mentioned that $\operatorname{det} \bar{\rho}_{\mathfrak{B}} \equiv \zeta \varepsilon_{p} \bmod \varpi$. Let $R_{\bar{\rho}_{\mathfrak{B}}}^{\mathrm{ps}, \zeta \varepsilon_{p}}$ be the deformation ring parametrising all of the 2-dimensional continuous determinants (in the sense of [Che14]) of $G_{\mathbb{Q}_{p}}$ lifting $\left(\operatorname{tr} \bar{\rho}_{\mathfrak{B}}\right.$, det $\left.\bar{\rho}_{\mathfrak{B}}\right)$ with (usual) determinant $\zeta \varepsilon_{p}$. Here is a summary of results of Paškūnas and Paškūnas-Tung that we will use later.

Theorem 6.3.3. Suppose $\mathfrak{B}$ contains an absolutely irreducible representation. Let $Z_{\mathfrak{B}}$ be the Bernstein centre of $\operatorname{Mod}_{G, \zeta}^{1 \mathrm{adm}}(\mathcal{O})[\mathfrak{B}]$.

(1) There is a finite $\mathcal{O}$-algebra homomorphism

$$
R_{\bar{\rho}_{\mathfrak{B}}}^{\mathrm{ps} \zeta \varepsilon_{p}} \rightarrow Z_{\mathfrak{B}}
$$

compatible with Colmez's functor (see [PT21, Theorem 1.2] for the precise statement). Hence, $\operatorname{Mod}_{G, \zeta}^{1 \operatorname{adm}}(\mathcal{O})[\mathfrak{B}]$ admits a $R_{\bar{\rho}_{\mathfrak{B}}}^{\mathrm{ps}, \zeta \varepsilon_{p}}$-module structure from this map.

(2) There is a faithful $R_{\bar{\rho}_{\mathfrak{B}}}^{\mathrm{ps}, \zeta \varepsilon_{p}}$-linear contravariant exact functor $\mathrm{m}$ from $\operatorname{Mod}_{G, \zeta}^{\mathrm{ladm}}(\mathcal{O})[\mathfrak{B}]$ to the category of $R_{\bar{\rho}_{\mathfrak{B}}}^{\mathrm{ps}, \zeta \varepsilon_{p}}$-modules sending admissible representations to finitely generated $R_{\bar{\rho}_{\mathfrak{B}}}^{\mathrm{ps} \zeta \varepsilon_{p}}$. modules.

Proof. Both claims follow from [PT21, Theorem 1.2, 1.3]. In fact, Paškūnas and Tung show that

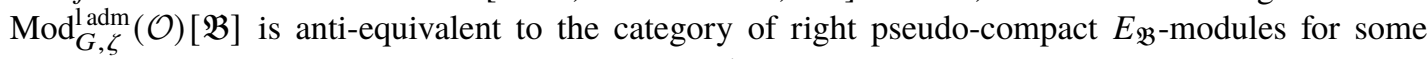
pseudo-compact ring $E_{\mathfrak{B}}$ and construct a map $R_{\bar{\rho}_{\mathfrak{B}}}^{\mathrm{ps}, \zeta \varepsilon_{p}} \rightarrow Z_{\mathfrak{B}} \subseteq E_{\mathfrak{B}}$ which makes $E_{\mathfrak{B}}$ into a finitely generated $R_{\bar{\rho}_{\mathfrak{B}}}^{\mathrm{ps}, \zeta \varepsilon_{p}}$-module. The functor $\mathrm{m}$ is defined by passing the action of $E_{\mathfrak{B}}$ to $R_{\bar{\rho}_{\mathfrak{B}}}^{\mathrm{ps}, \zeta \varepsilon_{p}}$.

6.3.4. Next we turn to the global side. Fix a tame level $K^{p}$ from now on. Let $m$ be a maximal ideal of $\mathbb{T}\left(K^{p}\right) \otimes_{\mathbb{Z}_{p}} \mathcal{O}$. We may assume the residue field of $m$ is $\mathbb{F}$ by enlarging $E$. Recall that there is a 2-dimensional determinant of $G_{\mathbb{Q}}$ valued in $\mathbb{T}\left(K^{p}\right)$ and we extend it to a determinant valued in $\mathbb{T}\left(K^{p}\right) \otimes_{\mathbb{Z}_{p}} \mathcal{O}$. Denote by $D_{p}$ its restriction to $G_{\mathbb{Q}_{p}}$ and by $\bar{D}_{p}$ its reduction modulo $\mathrm{m}$. Again enlarging $E$ if necessary, we may assume that $\bar{D}_{p}$ arises from a 2-dimensional semi-simple representation 
$\bar{\rho}_{\mathfrak{m}, p}: G_{\mathbb{Q}_{p}} \rightarrow \mathrm{GL}_{2}(\mathbb{F})$. Fix a continuous character $\zeta^{\prime}: \mathbb{A}_{f}^{\times} / \operatorname{det}\left(K^{p}\right) \mathbb{Q}_{>0}^{\times} \rightarrow \mathcal{O}^{\times}$such that $\zeta=\left.\zeta^{\prime}\right|_{\mathbb{Q}_{p}^{\times}}:$ $\mathbb{Q}_{p}^{\times} \rightarrow \mathcal{O}^{\times}$is congruent to $\left(\operatorname{det} \bar{\rho}_{\mathfrak{m}, p}\right) \omega^{-1}$ modulo $\varpi$ via local class field theory. For $?=E, \mathcal{O}, E / \mathcal{O}$, let $\tilde{H}^{1}\left(K^{p}, ?\right)_{\zeta^{\prime}, \mathfrak{m}}$ be the subspace of $\left(\tilde{H}^{1}\left(K^{p}, \mathbb{Z}_{p}\right) \otimes_{\mathbb{Z}_{p}} ?\right)_{\mathfrak{m}}$ on which the centre $\mathbb{A}_{f}^{\times}$acts via $\zeta^{\prime}$ and let $\mathbb{T}$ be the image of $\left(\mathbb{T}\left(K^{p}\right) \otimes \mathcal{O}\right)_{\mathfrak{m}}$ inside $\operatorname{End}\left(\tilde{H}^{1}\left(K^{p}, E / \mathcal{O}\right)_{\zeta^{\prime}, \mathfrak{m}}\right)$. Then $D_{p}$ induces a natural map

$$
R_{\bar{\rho}_{\mathrm{m}, p}}^{\mathrm{ps}, \zeta \varepsilon_{p}} \rightarrow \mathbb{T}
$$

Hence, the faithful Hecke action of $\mathbb{T}$ induces an action of $R_{\bar{\rho}_{\mathfrak{m}, p}}^{\mathrm{ps} \zeta \varepsilon_{p}}$ on $\tilde{H}^{1}\left(K^{p}, E / \mathcal{O}\right)_{\zeta^{\prime}, \mathrm{m}}$ by our discussion. We denote this action by $\tau^{p}$.

On the other hand, $\bar{\rho}_{\mathfrak{m}, p}$ determines a block $\mathfrak{B}_{\mathfrak{m}}$ of $\operatorname{Mod}_{G, \zeta}^{\mathrm{ladm}}(\mathcal{O})$. Now we can state the main result of this subsection.

Theorem 6.3.5. Let $\mathfrak{m}$ be a maximal ideal of $\mathbb{T}\left(K^{p}\right) \otimes_{\mathbb{Z}_{p}} \mathcal{O}$.

(1) $\tilde{H}^{1}\left(K^{p}, E / \mathcal{O}\right)_{\zeta^{\prime}, \mathfrak{m}} \in \operatorname{Mod}_{G, \zeta}^{\mathrm{ladm}}(\mathcal{O})\left[\mathfrak{B}_{\mathfrak{m}}\right]$. Hence, by Theorem 6.3.3, there is a natural action $\tau_{p}$ of $R_{\bar{\rho}_{\mathfrak{B}_{\mathfrak{m}}}}^{\mathrm{ps}, \zeta \varepsilon_{p}}=R_{\bar{\rho}_{\mathfrak{m}, p}}^{\mathrm{ps}, \zeta \varepsilon_{p}}$ on $\tilde{H}^{1}\left(K^{p}, E / \mathcal{O}\right)_{\zeta^{\prime}, \mathrm{m}}$.

(2) Two actions $\tau^{p}$ and $\tau_{p}$ of $R_{\bar{\rho}_{\mathrm{m}, p}}^{\mathrm{ps} \zeta \varepsilon_{p}}$ are the same.

Before giving a proof, we show that this result implies a (weak) form of local-global compatibility which will be enough for our applications. Recall that by [CDP14, Theorem 1.1], there is a bijection between the isomorphism classes of the following two sets:

- 2-dimensional absolutely irreducible representation of $G_{\mathbb{Q}_{p}}$ over $E$;

○ nonordinary admissible unitary $E$-Banach representations of $\mathrm{GL}_{2}\left(\mathbb{Q}_{p}\right)$.

Here, a Banach representation is nonordinary if it is not a subquotient of a parabolic induction of a unitary character. This bijection is compatible with taking finite extensions of $E$. Hence, we can extend this bijection $\overline{\mathbb{Q}}_{p}$-linearly to a map (not bijection) from the isomorphism classes of 2-dimensional irreducible representation of $G_{\mathbb{Q}_{p}}$ over $\overline{\mathbb{Q}}_{p}$ to the isomorphism classes of $\overline{\mathbb{Q}}_{p}$-representations of $\mathrm{GL}_{2}\left(\mathbb{Q}_{p}\right)$. We denote this map by $\rho \mapsto \Pi(\rho)$.

Corollary 6.3.6. Suppose $\rho=\rho_{\lambda}$ is pro-modular and irreducible for some tame level $K^{p}$. Then $\tilde{H}^{1}\left(K^{p}, \overline{\mathbb{Q}}_{p}\right)\left[\mathfrak{p}_{\lambda}\right] \neq 0$; that is, $\rho$ is pro-cohomological. Moreover, we have the following description of $\tilde{H}^{1}\left(K^{p}, \overline{\mathbb{Q}}_{p}\right)\left[\mathfrak{p}_{\lambda}\right]$ as a representation of $\mathrm{GL}_{2}\left(\mathbb{Q}_{p}\right)$ :

○ If $\left.\rho\right|_{G_{\mathbb{Q}_{p}}}$ is irreducible, then $\tilde{H}^{1}\left(K^{p}, \overline{\mathbb{Q}}_{p}\right)\left[\mathfrak{p}_{\lambda}\right] \cong \Pi\left(\left.\rho\right|_{G_{Q_{p}}}\right)^{\oplus d}$ for some d.

○ If $\left.\rho\right|_{G_{\mathbb{Q} p}}$ is reducible, then $\tilde{H}^{1}\left(K^{p}, \overline{\mathbb{Q}}_{p}\right)\left[\mathfrak{p}_{\lambda}\right]$ has an ordinary subrepresentation.

Remark 6.3.7. For later application (Theorem 6.4.7), all we need is that $\tilde{H}^{1}\left(K^{p}, \overline{\mathbb{Q}}_{p}\right)\left[\mathfrak{p}_{\lambda}\right]$ is nonzero and contains an irreducible admissible representation.

Proof. We denote by $\mathrm{m}_{0}$ the $R_{\bar{\rho}_{\mathrm{m}, p}}^{\mathrm{ps}, \zeta \varepsilon}$-module obtained by applying the faithful functor $\mathrm{m}$ in Theorem 6.3.3 to $\tilde{H}^{1}\left(K^{p}, E / \mathcal{O}\right)_{\zeta^{\prime}, \mathrm{m}}$. Then the admissibility of $\tilde{H}^{1}\left(K^{p}, E / \mathcal{O}\right)_{\zeta^{\prime}, \mathrm{m}}$ implies that $\mathrm{m}_{0}$ is a finitely generated $R_{\bar{\rho}_{\mathfrak{m}, p}}^{\mathrm{ps} \zeta \varepsilon}$-module. Since the action $\tau_{p}$ agrees with $\tau^{p}$ which factors through $\mathbb{T}$ by Theorem 6.3.5, we conclude that $\mathrm{m}_{0}$ is a faithful finitely generated $\mathbb{T}$-module. In particular, $\mathrm{m}_{0} \otimes_{\mathbb{T}} k(\mathfrak{p}) \neq 0$ for any $\mathfrak{p} \in \operatorname{Spec} \mathbb{T}$, where $k(\mathfrak{p})$ denotes the residue field of $\mathfrak{p}$. From this, we easily deduce $\tilde{H}^{1}\left(K^{p}, \overline{\mathbb{Q}}_{p}\right)\left[\mathfrak{p}_{\lambda}\right] \neq 0$ in the corollary. Using $\tau^{p}=\tau_{p}$, our description of $\tilde{H}^{1}\left(K^{p}, \overline{\mathbb{Q}}_{p}\right)\left[\mathfrak{p}_{\lambda}\right]$ follows from Paškūnas's work on Banach representations [Paš13, Theorem 1.10, 1.11], [Paš16, Corollary 2.24].

Proof of Theorem 6.3.5. We can replace $K^{p}$ by an open subgroup and assume $K^{p} \mathrm{GL}_{2}\left(\mathbb{Z}_{p}\right)$ is sufficiently small. After twisting a character of $\mathbb{A}_{f}^{\times} / \mathbb{Q}_{>0}^{\times}$, we may assume $\zeta(x)=x^{k}, x \in \mathbb{Z}_{p}^{\times}$for some integer $k$. We denote by $\tilde{H}^{1}\left(K^{p}, \mathbb{Q}_{p}\right)_{k} \subset \tilde{H}^{1}\left(K^{p}, \mathbb{Q}_{p}\right)$ the subspace on which the centre $\mathbb{Z}_{p}^{\times} \subset \mathrm{GL}_{2}\left(\mathbb{Q}_{p}\right)$ acts via $k$ th 
power. It has a norm induced from $\tilde{H}^{1}\left(K^{p}, \mathbb{Z}_{p}\right)$. Then after enlarging $E$ if necessary, there is a natural decomposition

$$
\tilde{H}^{1}\left(K^{p}, \mathbb{Q}_{p}\right)_{k} \otimes_{\mathbb{Q}_{p}} E=\bigoplus_{\left(\mathfrak{m}^{\prime}, \zeta^{\prime \prime}\right)} \tilde{H}^{1}\left(K^{p}, E\right)_{\zeta^{\prime \prime}, \mathfrak{m}^{\prime}},
$$

where $\mathfrak{m}^{\prime}$ runs through all maximal ideals of $\mathbb{T}\left(K^{p}\right) \otimes \mathcal{O}$ and $\zeta^{\prime \prime}: \mathbb{A}_{f}^{\times} / \operatorname{det}\left(K^{p}\right) \mathbb{Q}_{>0}^{\times}$runs through all continuous characters satisfying $\zeta^{\prime \prime}(x)=x^{k}, x \in \mathbb{Z}_{p}^{\times}$. In particular, $\left(\mathrm{m}, \zeta^{\prime}\right)$ appears inside this decomposition. Note that there are only finitely many $\left(\mathfrak{m}^{\prime}, \zeta^{\prime \prime}\right)$.

Lemma 6.3.8. The $\mathrm{GL}_{2}\left(\mathbb{Z}_{p}\right)$-algebraic vectors in $\tilde{H}^{1}\left(K^{p}, \mathbb{Q}_{p}\right)_{k}$ are dense in $\tilde{H}^{1}\left(K^{p}, \mathbb{Q}_{p}\right)_{k}$.

Lemma 6.3.9. The Hecke action of $\mathbb{T}\left(K^{p}\right)$ on the $\mathrm{GL}_{2}\left(\mathbb{Z}_{p}\right)$-algebraic vectors in $\tilde{H}^{1}\left(K^{p}, \mathbb{Q}_{p}\right)$ is semisimple.

We will give proofs of both lemmas at the end. Assuming these results, we deduce from the natural decomposition above that the $\mathrm{GL}_{2}\left(\mathbb{Z}_{p}\right)$-algebraic vectors in $\tilde{H}^{1}\left(K^{p}, E\right)_{\zeta^{\prime}, \mathrm{m}}$ are dense. Now one can argue in exactly the same way as proof of Proposition 5.5 of [Pas 18]. We only give a sketch here. It should be mentioned that these algebraic vectors correspond to cohomology of certain standard local systems

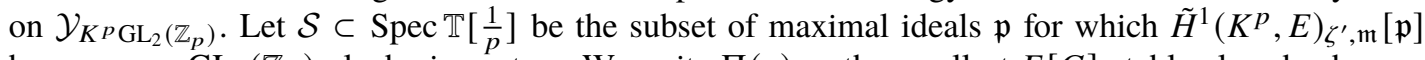
has nonzero $\mathrm{GL}_{2}\left(\mathbb{Z}_{p}\right)$-algebraic vectors. We write $\Pi(\mathfrak{p})$ as the smallest $E[G]$-stable closed subspace containing the $\mathrm{GL}_{2}\left(\mathbb{Z}_{p}\right)$-algebraic vectors in $\tilde{H}^{1}\left(K^{p}, E\right)_{\zeta^{\prime}, \mathfrak{m}}[\mathfrak{p}]$. Let $\Pi(\mathfrak{p})^{o} \subset \Pi(\mathfrak{p})$ be its open unit ball. By our density result and the semi-simplicity of Hecke action on the $\mathrm{GL}_{2}\left(\mathbb{Z}_{p}\right)$-algebraic vectors in $\tilde{H}^{1}\left(K^{p}, E\right)_{\zeta^{\prime}, \mathfrak{m}}$, it suffices to prove that for any $\mathfrak{p} \in \mathcal{S}$,

$$
\Pi(\mathfrak{p}) / \Pi(\mathfrak{p})^{o} \in \operatorname{Mod}_{G, \zeta}^{\operatorname{ladm}}(\mathcal{O})\left[\mathfrak{B}_{\mathfrak{m}}\right]
$$

and both actions $\tau^{p}, \tau_{p}$ are the same on it. Both claims are really formal consequences of classical localglobal compatibility and uniqueness of the universal unitary completion in this case ([BB10, Corollarie 5.3.4], [BE10, Proposition 2.2.1]) and the compatibility between $p$-adic and classical local Langlands correspondence [CDP14, Theorem 1.3]. This finishes the proof of Theorem 6.3.5.

Proof of Lemma 6.3.8. As pointed out by a referee, the following argument was sketched by Matthew Emerton in [Eme11, Remark 5.4.2].

We begin the proof by recalling a complex computing $\tilde{H}^{i}\left(K^{p}, \mathbb{Q}_{p}\right)$. Let $\tilde{H}_{c}^{i}\left(K^{p}, \mathbb{Z}_{p}\right)$ be the completed cohomology with compact support, which is defined by

$$
\tilde{H}_{c}^{i}\left(K^{p}, \mathbb{Z}_{p}\right):={\underset{\lim }{\longleftarrow}}_{\overrightarrow{K_{p}}} \lim _{c} H_{c}^{i}\left(Y_{K^{p} K_{p}}(\mathbb{C}), \mathbb{Z} / p^{n}\right)
$$

Since $Y_{K^{p} K_{p}}$ is a noncompact Riemann surface, it is easy to see that $\tilde{H}_{c}^{i}\left(K^{p}, \mathbb{Z}_{p}\right)=0, i \neq 1$. There is a spectral sequence (coming from the Poincaré duality) relating $\tilde{H}^{\bullet}$ and $\tilde{H}_{c}^{1}$ (Subsection 1.3 of [CE12]) as follows. Let $\Lambda$ be the completed group $\operatorname{ring} \mathbb{Z}_{p}\left[\left[\mathrm{GL}_{2}\left(\mathbb{Z}_{p}\right)\right]\right]$. Then $\tilde{H}_{c}^{1}\left(K^{p}, \mathbb{Z}_{p}\right)^{d}:=$ $\operatorname{Hom}_{\mathbb{Z}_{p}}\left(\tilde{H}_{c}^{1}\left(K^{p}, \mathbb{Z}_{p}\right), \mathbb{Z}_{p}\right)$ admits a resolution by finite free $\Lambda$-modules:

$$
\cdots \rightarrow \Lambda^{\oplus d_{-1}} \rightarrow \Lambda^{\oplus d_{0}} \rightarrow \Lambda^{\oplus d_{1}} \rightarrow \tilde{H}_{c}^{1}\left(K^{p}, \mathbb{Z}_{p}\right)^{d} \rightarrow 0 .
$$

Taking $\operatorname{Hom}_{\Lambda}(\bullet, \Lambda)$ of this resolution, where $\Lambda$ is viewed as a left module of itself, we obtain a complex of right $\Lambda$-modules (using the right $\Lambda$-module structure of $\Lambda$ )

$$
\cdots \leftarrow \Lambda^{\oplus d_{-1}} \leftarrow \Lambda^{\oplus d_{0}} \leftarrow \Lambda^{\oplus d_{1}} .
$$


We can view this sequence as a complex of left $\Lambda$-modules by applying the main involution $g \mapsto g^{-1}$ of $\Lambda$. Now taking $\operatorname{Hom}_{\mathbb{Z}_{p}}^{\text {cont }}\left(\bullet, \mathbb{Q}_{p}\right)$, we get a $\mathrm{GL}_{2}\left(\mathbb{Z}_{p}\right)$-equivariant complex

$$
C^{\bullet}=\cdots \rightarrow \mathscr{C}\left(\mathrm{GL}_{2}\left(\mathbb{Z}_{p}\right), \mathbb{Q}_{p}\right)^{\oplus d_{-1}} \rightarrow \mathscr{C}\left(\mathrm{GL}_{2}\left(\mathbb{Z}_{p}\right), \mathbb{Q}_{p}\right)^{\oplus d_{0}} \rightarrow \mathscr{C}\left(\mathrm{GL}_{2}\left(\mathbb{Z}_{p}\right), \mathbb{Q}_{p}\right)^{\oplus d_{1}}
$$

where $\mathscr{C}\left(\mathrm{GL}_{2}\left(\mathbb{Z}_{p}\right), \mathbb{Q}_{p}\right)$ denotes the space of $\mathbb{Q}_{p}$-valued continuous functions on $\mathrm{GL}_{2}\left(\mathbb{Z}_{p}\right)$. The result in Subsection 1.3 of [CE12] says that this complex computes $\tilde{H}^{i}\left(K^{p}, \mathbb{Q}_{p}\right)$ as a $\mathrm{GL}_{2}\left(\mathbb{Z}_{p}\right)$-representation. For a $\mathbb{Z}_{p}\left[\mathrm{GL}_{2}\left(\mathbb{Z}_{p}\right)\right]$-module $M$, we denote by $M_{k}$ the subspace on which the centre $\mathbb{Z}_{p}^{\times}$acts via $k$ th power. We claim that

$$
C_{k}^{\bullet}=\cdots \rightarrow \mathscr{C}\left(\mathrm{GL}_{2}\left(\mathbb{Z}_{p}\right), \mathbb{Q}_{p}\right)_{k}^{\oplus d_{-1}} \rightarrow \mathscr{C}\left(\mathrm{GL}_{2}\left(\mathbb{Z}_{p}\right), \mathbb{Q}_{p}\right)_{k}^{\oplus d_{0}} \rightarrow \mathscr{C}\left(\mathrm{GL}_{2}\left(\mathbb{Z}_{p}\right), \mathbb{Q}_{p}\right)_{k}^{\oplus d_{1}}
$$

computes $\tilde{H}^{i}\left(K^{p}, \mathbb{Q}_{p}\right)_{k}$. Assuming this, $\tilde{H}^{1}\left(K^{p}, \mathbb{Q}_{p}\right)_{k}$ is a $\mathrm{GL}_{2}\left(\mathbb{Z}_{p}\right)$-equivariant quotient of $\mathscr{C}\left(\mathrm{GL}_{2}\left(\mathbb{Z}_{p}\right), \mathbb{Q}_{p}\right)_{k}^{\oplus d_{1}}$. Hence, we get the density statement in Lemma 6.3.8 because $\mathrm{GL}_{2}\left(\mathbb{Z}_{p}\right)$-algebraic vectors are dense in $\mathscr{C}\left(\mathrm{GL}_{2}\left(\mathbb{Z}_{p}\right), \mathbb{Q}_{p}\right)_{k}$ ([Pan19, Proposition 3.2.9]).

To see that $C_{k}^{\bullet}$ computes $\tilde{H}^{i}\left(K^{p}, \mathbb{Q}_{p}\right)_{k}$, it is enough to show that

$$
H_{\text {cont }}^{j}\left(\mathbb{Z}_{p}^{\times}, \mathscr{C}\left(\mathrm{GL}_{2}\left(\mathbb{Z}_{p}\right), \mathbb{Q}_{p}\right) \otimes z^{-k}\right)=H_{\text {cont }}^{j}\left(\mathbb{Z}_{p}^{\times}, \tilde{H}^{0}\left(K^{p}, \mathbb{Q}_{p}\right) \otimes z^{-k}\right)=0, j \geq 1,
$$

where $z^{-k}=\mathbb{Q}_{p}$ with $\mathbb{Z}_{p}^{\times}$acting via $(-k)$ th power. Since everything is over a characteristic zero field, it suffices to prove this after replacing $\mathbb{Z}_{p}^{\times}$by an open subgroup. Let $H=1+p^{2} \mathbb{Z}_{p} \subset \mathbb{Z}_{p}^{\times}$. Using the description of $\tilde{H}^{0}\left(K^{p}, \mathbb{Q}_{p}\right)$ in [Eme06b, 4.2], it is easy to see that as Banach representations of $H$, both $\mathscr{C}\left(\mathrm{GL}_{2}\left(\mathbb{Z}_{p}\right), \mathbb{Q}_{p}\right)$ and $\tilde{H}^{0}\left(K^{p}, \mathbb{Q}_{p}\right)$ are of the form $\mathscr{C}\left(H \times R, \mathbb{Q}_{p}\right)$ for some profinite set $R$ with trivial $H$-action. Hence, their tensor products with $z^{-k}$ have no higher cohomology.

Proof of Lemma 6.3.9. By Emerton's description [Eme06b, 4.3.4] of the $\mathrm{GL}_{2}\left(\mathbb{Z}_{p}\right)$-algebraic vectors in $\tilde{H}^{1}\left(K^{p}, \mathbb{Q}_{p}\right)$, it suffices to show that the Hecke action on $H^{1}\left(Y_{K^{p} \mathrm{GL}_{2}\left(\mathbb{Z}_{p}\right)}(\mathbb{C}), \mathcal{V}_{\mathcal{W}}\right)$ is semi-simple for any irreducible algebraic representation $W$ of $\mathrm{GL}_{2}$ over $\mathbb{Q}_{p}$, where $\mathcal{V}_{\mathcal{W}}$ denotes the local system corresponding to $W$. This result is presumably well-known for experts. We sketch a proof here. We may change the coefficient field of the local system to $\mathbb{C}$. Then using the Shimura isomorphism, it suffices to prove the semi-simplicity of the Hecke action on the space of classical modular forms. This is clear for cusp forms because $T_{l}, l \notin S$ are normal operators with respect to the Petersson inner product. The theory of Eisenstein series then provides an eigenbasis for the orthogonal complement of cusp forms. See, for example, Weisinger's thesis [Wei77, Chap. 1].

Here is a more conceptual argument suggested to me by Matthew Emerton using polarisations. For simplicity, we assume $W$ is the trivial local system. But the argument can be generalised for any $W$. Let $Y=Y_{K^{p} \mathrm{GL}_{2}\left(\mathbb{Z}_{p}\right)}(\mathbb{C})$ and $X=X_{K^{p} \mathrm{GL}_{2}\left(\mathbb{Z}_{p}\right)}(\mathbb{C})$. By the Poincaré duality, it is enough to consider $H_{c}^{1}(Y, \mathbb{C})$. There is a natural exact sequence

$$
\mathbb{C}^{\oplus|\mathcal{C}|} \rightarrow H_{c}^{1}(Y, \mathbb{C}) \rightarrow H^{1}(X, \mathbb{C}) \rightarrow 0 .
$$

(Recall that $\mathcal{C}$ denotes the set of cusps in $X$.) The Hecke operators $T_{l}, l \notin S$ are normal on $H^{1}(X, \mathbb{C})$ (respectively $\mathbb{C}^{\oplus|\mathcal{C}|}$ ) with respect to the Riemann form (respectively standard Hermitian form on $\mathbb{C}^{\oplus|\mathcal{C}|}$ ). Hence, the Hecke actions of $\mathbb{T}\left(K^{p}\right)$ on both spaces are semi-simple. Now our claim follows from the Manin-Drinfeld theorem as it implies that $H_{c}^{1}(Y, \mathbb{C}) \rightarrow H^{1}(X, \mathbb{C})$ has a natural splitting; cf. [Elk90].

\subsection{Colmez's Kirillov model}

6.4.1. Let $\rho=\rho_{\lambda}$ be as in Corollary 6.3.6. Suppose $L$ is a finite extension of $\mathbb{Q}_{p}$ containing $\lambda\left(\mathbb{T}\left(K^{p}\right)\right)$. Then Corollary 6.3.6 implies that $\tilde{H}^{1}\left(K^{p}, L\right)\left[\mathfrak{p}_{\lambda}\right]=\tilde{H}^{1}\left(K^{p}, \overline{\mathbb{Q}}_{p}\right)\left[\mathfrak{p}_{\lambda}\right] \cap \tilde{H}^{1}\left(K^{p}, L\right)$ contains an irreducible admissible Banach representation $\Pi$ of $\mathrm{GL}_{2}\left(\mathbb{Q}_{p}\right)$ over $L$. To apply our previous results 
(Theorem 5.4.2, Theorem 6.2.2), it is crucial to know whether its locally analytic vectors $\Pi^{\mathrm{la}}$ admit nonzero $n$-invariants and whether its $N_{0}$-invariants have a $U_{p}$-eigenvector. Thanks to the beautiful work of Colmez, both questions have affirmative answers by his Kirillov model [Col10, Chap. VI]. We first give a brief review of his work. A nice summary can be found in Subsections 7.3 and 7.4 of [DLB17]. I would like to thank Matthew Emerton for sharing his unpublished notes [EPW] with Robert Pollack and Tom Weston on this subject.

6.4.2. Let $E$ be a finite extension of $\mathbb{Q}_{p}$ and $V$ a 2-dimensional representation of $G_{\mathbb{Q}_{p}}$ over $E$. By Fontaine's work [Fon04], we can study $V \otimes_{\mathbb{Q}_{p}} B_{\mathrm{dR}}^{+}$by Sen's method. As a result, we obtain a free $E[[t]] \otimes_{\mathbb{Q}_{p}} \mathbb{Q}_{p}\left(\mu_{p^{\infty}}\right)$-module $D_{\text {dif }}^{+}(V)$ of rank 2 equipped with a semi-linear action of $\Gamma=\operatorname{Gal}\left(\mathbb{Q}_{p}\left(\mu_{p^{\infty}}\right) / \mathbb{Q}_{p}\right)$. Let $D_{\text {dif }}(V):=D_{\text {dif }}^{+}(V)\left[\frac{1}{t}\right]$ and

$$
D_{\text {dif }}^{-}(V):=D_{\text {dif }}(V) / D_{\text {dif }}^{+}(V),
$$

a torsion $\mathbb{Q}_{p}\left(\mu_{p^{\infty}}\right)[t]$-module. We denote by $\mathscr{C}_{c}\left(\mathbb{Q}_{p}^{\times}, D_{\text {dif }}^{-}(V)\right)^{\Gamma}$ the space of functions $\phi: \mathbb{Q}_{p}^{\times} \rightarrow$ $D_{\text {dif }}^{-}(V)$ with compact support satisfying

$$
\phi(a x)=\sigma_{a}(\phi(x)), a \in \mathbb{Z}_{p}^{\times}, x \in \mathbb{Q}_{p}^{\times},
$$

where $\sigma_{a} \in \Gamma$ is inverse image of $a$ under $\varepsilon_{p}: \Gamma \stackrel{\sim}{\rightarrow} \mathbb{Z}_{p}^{\times}$. In particular, $\phi$ is completely determined by $\left\{\phi\left(p^{n}\right)\right\}_{n \in \mathbb{Z}}$. Let $P=\left(\begin{array}{cc}\mathbb{Q}_{p}^{\times} & \mathbb{Q}_{p} \\ 0 & 1\end{array}\right)$, the mirabolic subgroup of $\mathrm{GL}_{2}\left(\mathbb{Q}_{p}\right)$. One can define an action of $P$ on $\mathscr{C}_{c}\left(\mathbb{Q}_{p}^{\times}, D_{\text {dif }}^{-}(V)\right)^{\Gamma}$ by the formula

$$
\left(\left(\begin{array}{ll}
a & b \\
0 & 1
\end{array}\right) \phi\right)(x)=\varepsilon^{\prime}(b x) e^{t b x} \phi(a x),
$$

where $\varepsilon^{\prime}: \mathbb{Q}_{p} \rightarrow \mu_{p^{\infty}}$ is an additive character with kernel $\mathbb{Z}_{p}$ and $e^{t b x}$ is understood as $\sum_{n \geq 0} \frac{(b x)^{n}}{n !} t^{n}$. (One can view $\mathbb{Q}_{p} \rightarrow\left(\mathbb{Q}_{p}[[t]] \otimes_{\mathbb{Q}_{p}} \mathbb{Q}_{p}\left(\mu_{p^{\infty}}\right)\right)^{\times}, x \mapsto \varepsilon^{\prime}(x) e^{t x}$ as an additive character.) This is Colmez's Kirillov model.

Now we assume

○ $V$ is absolutely irreducible.

We denote by $\Pi(V)_{E}$ the irreducible unitary $E$-Banach representation of $\mathrm{GL}_{2}\left(\mathbb{Q}_{p}\right)$ associated to $V$ via the $p$-adic local Langlands correspondence ([CDP14, Theorem 1.1]) and by $\Pi(V)_{E}^{\text {la }}$ its locally analytic vectors.

Theorem 6.4.3. There is a P-equivariant embedding $\mathscr{C}_{c}\left(\mathbb{Q}_{p}^{\times}, D_{\mathrm{dif}}^{-}(V)\right)^{\Gamma} \rightarrow \Pi(V)_{E}^{\mathrm{la}}$.

Proof. See Proposition 7.6 of [DLB17].

The following corollary and remark were first pointed out to me by Matthew Emerton.

Corollary 6.4.4. $\Pi(V)_{E}^{\mathrm{la}, N_{0}} \neq 0$ and has a nonzero vector annihilated by $U_{p}$.

Proof. This is a standard formal consequence of the Kirillov model. More precisely, let $v \in D_{\text {dif }}^{-}(V)$ be a nonzero element annihilated by $t$. Consider $\phi \in \mathscr{C}_{c}\left(\mathbb{Q}_{p}^{\times}, D_{\text {dif }}^{-}(V)\right)^{\Gamma}$ defined by $\phi(1)=v$ and $\phi(x)=0, x \notin \mathbb{Z}_{p}^{\times}$. Clearly, $\phi$ is fixed by $\left(\begin{array}{ll}1 & 1 \\ 0 & 1\end{array}\right)$. Moreover,

$$
\left(U_{p} \cdot \phi\right)(x)=\sum_{i=0}^{p-1}\left(\left(\begin{array}{ll}
p & i \\
0 & 1
\end{array}\right) \phi\right)(x)=\sum_{i=0}^{p-1} \varepsilon^{\prime}(i x) \phi(p x) \text {. }
$$


If $p x \notin \mathbb{Z}_{p}^{\times}$, this is zero for trivial reason. If $x=\frac{1}{p}$, this is essentially the sum over all $p$ th roots of unity, which is again zero. Hence, $\phi$ is annihilated by $U_{p}$.

Remark 6.4.5. It is easy to see that all the vectors in $\mathscr{C}_{c}\left(\mathbb{Q}_{p}^{\times}, D_{\text {dif }}^{-}(V)\right)^{\Gamma, N_{0}}$ annihilated by $U_{p}$ (which we denote by $\left.\mathscr{C}_{c}\left(\mathbb{Q}_{p}^{\times}, D_{\text {dif }}^{-}(V)\right)^{\Gamma, N_{0}, U_{p}=0}\right)$ are of this form; that is,

$$
\mathscr{C}_{c}\left(\mathbb{Q}_{p}^{\times}, D_{\mathrm{dif}}^{-}(V)\right)^{\Gamma, N_{0}, U_{p}=0}=D_{\mathrm{dif}}^{+}(V) \cdot \frac{1}{t} / D_{\mathrm{dif}}^{+}(V)=D_{\mathrm{Sen}}(V(-1)) .
$$

Here $D_{\text {Sen }}(V(-1))$ is a free $E \otimes_{\mathbb{Q}_{p}} \mathbb{Q}_{p}\left(\mu_{p^{\infty}}\right)$-module of rank 2 equipped with a semi-linear action of $\Gamma$ associated to $V(-1)$ in Sen's theory.

In Colmez's work, it is also possible to describe the image of $\mathscr{C}_{c}\left(\mathbb{Q}_{p}^{\times}, D_{\text {dif }}^{-}(V)\right)^{\Gamma}$ inside $\Pi(V)_{E}^{\text {la }}$. In fact, one can show that this image contains $\left(\Pi(V)_{E}^{\text {la }}\right)^{N_{0}, U_{p}=0}$. Hence,

$$
\Pi(V)_{E}^{\mathrm{la}, N_{0}, U_{p}=0} \cong D_{\mathrm{Sen}}(V(-1)),
$$

which identifies the action of $\left(\begin{array}{cc}\mathbb{Z}_{p}^{\times} & 0 \\ 0 & 1\end{array}\right)$ on the left-hand side with the action of $\Gamma$ on the right-hand side. Therefore, let $a, b$ be the Hodge-Tate-Sen weights of $V$. Since $\Pi(V)$ has central character $\operatorname{det}(V) \varepsilon_{p}^{-1}$ ([CDP14, Corollary 1.2]), we conclude that both

$$
\left(\Pi(V)_{E}^{\mathrm{la}}\right)_{(-a-1,-b)},\left(\Pi(V)_{E}^{\mathrm{la}}\right)_{(-b-1,-a)}
$$

are nonzero. Moreover, let $P_{0}=\left(\begin{array}{cc}\mathbb{Z}_{p}^{\times} & \mathbb{Z}_{p} \\ 0 & 1\end{array}\right)$. We have

$$
\operatorname{dim}_{E} \Pi(V)_{E}^{\mathrm{la}, P_{0}, U_{p}=0}=\operatorname{dim}_{E}\left(V(-1) \otimes_{\mathbb{Q}_{p}} C\right)^{G_{Q_{p}}},
$$

which gives a representation-theoretic way to determine whether $V(-1)$ is Hodge-Tate of weight 0,0 or not. We will use this to give another proof of Corollary 6.4.9.

Corollary 6.4.6. Suppose $\Pi$ is an irreducible admissible unitary Banach representation of $\mathrm{GL}_{2}\left(\mathbb{Q}_{p}\right)$ over some finite extension of $\mathbb{Q}_{p}$. Then $\Pi^{\mathrm{la}, N_{0}} \neq 0$ and has a nonzero $U_{p}$-eigenvector.

Proof. We may enlarge the field of coefficients $E$ and assume that $\Pi$ is absolutely irreducible by [DS13, Theorem 1.1]. If $\Pi$ is nonordinary, then this follows Corollary 6.4.4 as $\Pi=\Pi(V)_{E}$ for some absolutely irreducible Galois representation $V$ by [CDP14, Theorem 1.1]. If $\Pi$ is ordinary - that is, a subquotient of a parabolic induction of a unitary character - then up to twist by a character $\eta \circ$ det, the representation $\Pi$ is either trivial representation 1, unitary Steinberg representation $\widehat{S t}$ or $\Pi$ is a unitary parabolic induction of a unitary character. Recall that $\widehat{S t}=\left(\operatorname{Ind}_{B} \mathrm{GL}_{2}\left(\mathbb{Q}_{p}\right) \mathbf{1}\right) / \mathbf{1}$, the universal unitary completion of the usual Steinberg representation. In all of these cases, it is easy to check that $\Pi^{\mathrm{la}, N_{0}} \neq 0$ and has a nonzero $U_{p}$-eigenvector.

We obtain the following classicality result by combining Corollary 6.4.6, Corollary 6.3.6 and Theorem 6.2.2.

Theorem 6.4.7. Suppose $\rho=\rho_{\lambda}$ is pro-modular and irreducible. Assume

$\left.\circ \rho\right|_{G_{Q_{p}}}$ is Hodge-Tate of weight 0,0 .

Then $\rho$ is classical; that is, $M_{1}\left(K^{p}\right)\left[\mathfrak{p}_{\lambda}\right] \neq 0$ for some tame level $K^{p}$.

Proof. Suppose $\rho$ is pro-modular for some tame level $K^{p}$. We have

$$
\tilde{H}^{1}\left(K^{p}, \overline{\mathbb{Q}}_{p}\right)\left[\mathfrak{p}_{\lambda \cdot t}\right]=\tilde{H}^{1}\left(K^{p}, \overline{\mathbb{Q}}_{p}\right)\left[\mathfrak{p}_{\lambda}\right] \cdot t \neq 0
$$


by Corollary 6.3.6. Recall that $t \in \tilde{H}^{0}\left(K^{p}, \mathbb{Z}_{p}\right)$ is an invertible function, so $\tilde{H}^{1}\left(K^{p}, \overline{\mathbb{Q}}_{p}\right)\left[\mathfrak{p}_{\lambda}\right] \cdot t$ is understood as a subspace of $\tilde{H}^{1}\left(K^{p}, \overline{\mathbb{Q}}_{p}\right)$. Here $\lambda \cdot t$ was defined in proof of Theorem 6.2.2. Corollary 6.4.6 then implies that $\tilde{H}^{1}\left(K^{p}, C\right)^{\text {la }}\left[\mathfrak{p}_{\lambda \cdot t}\right]^{N_{0}, U_{p}=\alpha} \neq 0$ for some $\alpha \in \overline{\mathbb{Q}}_{p}$. By our result on the infinitesimal character (Proposition 6.1.5), $(h+1)^{2}=0$ on this space. Hence, the weight- $(0,1)$ subspace

$$
\tilde{H}^{1}\left(K^{p}, C\right)_{(0,1)}^{\mathrm{la}}\left[\mathfrak{p}_{\lambda \cdot t}\right]^{N_{0}, U_{p}=\alpha} \neq 0 .
$$

Now assume $\rho$ is not classical. As in the proof of Theorem 6.2.2, we have

$$
\tilde{H}^{1}\left(K^{p}, C\right)_{(0,1)}^{\mathrm{la}}\left[\mathfrak{p}_{\lambda \cdot t}\right]=M_{1}^{\dagger}\left(K^{p}\right)\left[\mathfrak{p}_{\lambda}\right] \cdot e_{1}^{-1} t
$$

However, this means that $M_{1}^{\dagger}\left(K^{p}\right)\left[\mathfrak{p}_{\lambda}\right]^{N_{0}, U_{p}=p \alpha} \neq 0$, which contradicts Theorem 6.2.2. Hence, $\rho$ has to be classical.

We quote the following result ([Eme11, Theorem 1.2.3], which is based on previous work of Böckle, Diamond-Flach-Guo, Khare-Wintenberger, Kisin) on promodularity of a Galois representation. For simplicity, we exclude the case $\left.\bar{\rho}\right|_{G_{Q_{p}}} \cong \eta \otimes\left(\begin{array}{cc}\omega & * \\ 0 & 1\end{array}\right)$, a nonsplit extension. As a corollary, we give a new proof of the Fontaine-Mazur conjecture in the irregular case under conditions below.

Theorem 6.4.8. Let $p>2$ be a prime number and $\rho: G_{\mathbb{Q}} \rightarrow \mathrm{GL}_{2}\left(\overline{\mathbb{Q}}_{p}\right)$ be a continuous 2-dimensional irreducible odd representation which is unramified outside of finitely many primes. Assume

(1) $\left.\bar{\rho}\right|_{G_{\mathbb{Q}\left(\mu_{p}\right)}}$ is irreducible;

(2) $\left(\left.\bar{\rho}\right|_{G_{Q_{p}}}\right)^{s s}$ is either irreducible or of the form $\eta_{1} \oplus \eta_{2}$ for some characters $\eta_{1}, \eta_{2}$ satisfying $\eta_{1} / \eta_{2} \neq$ $1, \omega^{ \pm 1}$.

Then $\rho$ is pro-modular.

Corollary 6.4.9. Let $\rho$ be as in Theorem 6.4.8. If $\rho$ is Hodge-Tate of weight 0,0 , then $\rho$ comes from a cuspidal eigenform of weight 1 .

Proof. A combination of the previous two results.

Remark 6.4.10. The assumptions in this result could possibly be removed in the following way. Let $\rho$ be a continuous 2-dimensional irreducible odd representation $\rho: G_{\mathbb{Q}, S} \rightarrow \mathrm{GL}_{2}\left(\overline{\mathbb{Q}}_{p}\right)$ which is unramified outside of finitely many primes. In [Pan19], it is proved that $\left.\rho\right|_{G_{F}}$ is irreducible and pro-modular for a solvable totally real field extension $F / \mathbb{Q}$ in which $p$ completely splits, under the assumption

$\circ p>2$;

○ If $p=3$, then $\left(\left.\bar{\rho}\right|_{G_{\mathbb{Q}_{p}}}\right)^{s s}$ is not of the form $\eta \oplus \eta \omega$.

Hence, one can prove Corollary 6.4.9 under these assumptions, if Theorem 6.4.7 can be extended to the case of Hilbert modular variety over a totally real field $F$ in which $p$ completely splits. It seems (at least to me) that it is reasonable to expect such a generalisation.

We can also determine the possible systems of Hecke eigenvalues appearing in spaces of overconvergent modular forms.

Theorem 6.4.11. Suppose $\rho=\rho_{\lambda}$ is irreducible and pro-modular for some tame level $K^{p}$. Let $a, b$ be the Hodge-Tate-Sen weights of $\left.\rho\right|_{G_{Q_{p}}}$.

(1) If $M_{\left(n_{1}, n_{2}\right)}^{\dagger}\left(K^{p}\right)\left[\mathfrak{p}_{\lambda}\right] \neq 0$, then $\left(n_{1}, n_{2}\right)=(-a,-b-1)$ or $(-b,-a-1)$. In particular, if $M_{k}^{\dagger}\left(K^{p}\right)\left[\mathfrak{p}_{\lambda}\right] \neq 0$ for some $k \in \mathbb{Z}$, then $\{a, b\}=\{0, k-1\}$.

(2) Conversely, if $\left.\rho\right|_{G_{Q_{p}}}$ is irreducible, then $M_{\left(n_{1}, n_{2}\right)}^{\dagger}\left(K^{p}\right)\left[\mathfrak{p}_{\lambda}\right] \neq 0$ for $\left(n_{1}, n_{2}\right)=(-a,-b-1)$ and $(-b,-a-1)$. 
Proof. To see the first part, if $M_{\left(n_{1}, n_{2}\right)}^{\dagger}\left(K^{p}\right)\left[\mathfrak{p}_{\lambda}\right] \neq 0$ and $n_{1} \neq n_{2}+1$, then by Theorem 5.4.2, we have $\tilde{H}^{1}\left(K^{p}, C\right)_{\left(n_{1}-1, n_{2}+1\right)}^{\mathrm{la}}\left[\mathfrak{p}_{\lambda}\right] \neq 0$. Hence, Proposition 6.1.5 implies that $\rho_{\lambda}$ has Hodge-Tate-Sen weights $-n_{1},-1-n_{2}$. The case $n_{1}=n_{2}+1$ can be proved in a similar way. We omit the details here.

For the second part, if $a=b=0$, the proof of Theorem 6.4.7 shows that $M_{(0,-1)}^{\dagger}\left(K^{p}\right)\left[\mathfrak{p}_{\lambda}\right] \neq 0$. For the general case $a=b$, one can twist by some $t^{a}$ as in Section 5.3.9 to reduce to the case $a=0$.

Now assume $a \neq b$. By Theorem 6.3.6 and Remark 6.4.5, both

$$
\tilde{H}^{1}\left(K^{p}, C\right)_{(-a-1,-b)}^{\mathrm{la}}\left[\mathfrak{p}_{\lambda}\right] \text { and } \tilde{H}^{1}\left(K^{p}, C\right)_{(-b-1,-a)}^{\mathrm{la}}\left[\mathfrak{p}_{\lambda}\right]
$$

are nonzero. By symmetry, it is enough to consider $\tilde{H}^{1}\left(K^{p}, C\right)_{(-a-1,-b)}^{\mathrm{la}}\left[\mathfrak{p}_{\lambda}\right]$. Theorem 5.4.2 provides a natural decomposition of this space according to the Hodge-Tate-Sen weights. Since $\tilde{H}^{1}\left(K^{p}, \overline{\mathbb{Q}}_{p}\right)\left[\mathfrak{p}_{\lambda}\right]$ is $\rho_{\lambda}(-1)$-isotypic, the Hodge-Tate-Sen weight- $(b+1)$ component of $\tilde{H}^{1}\left(K^{p}, C\right)_{(-a-1,-b)}^{\mathrm{la}}\left[\mathfrak{p}_{\lambda}\right]$ is nonzero. Hence, $M_{(-a,-b-1)}^{\dagger}\left(K^{p}\right)\left[\mathfrak{p}_{\lambda}\right] \neq 0$ if $-a \neq-b-1$ by Theorem 5.4.2 (i.e., $k \neq 2$ ). If $-a=-b-1$, then we claim we still have $M_{(-a-1,-b), w}\left[\mathfrak{p}_{\lambda}\right] \cong M_{(-a,-b-1)}^{\dagger}\left(K^{p}\right)\left[\mathfrak{p}_{\lambda}\right]$ (as $\mathbb{T}\left(K^{p}\right)$-modules). This is because the difference between $M_{(-a-1,-b), w}$ and $M_{(-a,-b-1)}^{\dagger}\left(K^{p}\right)$ is essentially $M_{0}\left(K^{p}\right) \cdot t^{-a}$, which can be decomposed as

$$
\bigoplus_{\mathfrak{q} \in \operatorname{Max}\left(\mathbb{T}\left(K^{p}\right)\left[\frac{1}{p}\right]\right)} M_{0}\left(K^{p}\right) \cdot t^{-a}[\mathfrak{q}]
$$

as a Hecke-module, and $M_{0}\left(K^{p}\right) \cdot t^{-a}\left[\mathfrak{p}_{\lambda}\right]=0$ since $M_{0}\left(K^{p}\right) \cdot t^{-a}\left[\mathfrak{p}_{\lambda^{\prime}}\right] \neq 0$ only when $\rho_{\lambda^{\prime}}$ is reducible. This proves the second part.

Remark 6.4.12. We sketch another proof of Theorem 6.4.7 in the nonordinary case (i.e., $\left.\rho\right|_{G_{Q_{p}}}$ is irreducible) without using our work on the Sen operator (Theorem 5.1.11). Suppose $\rho$ is not classical. Then as in the proof of Theorem 6.2.2, we have the following exact sequence by Theorem 5.4.6:

$$
0 \rightarrow M_{1}^{\dagger}\left(K^{p}\right)\left[\mathfrak{p}_{\lambda}\right] \cdot e_{1}^{-1} t \rightarrow \tilde{H}^{1}\left(K^{p}, C\right)_{(0,1)}^{\mathrm{la}}\left[\mathfrak{p}_{\lambda \cdot t}\right] \rightarrow M_{1}^{\dagger}\left(K^{p}\right)\left[\mathfrak{p}_{\lambda}\right] \cdot e_{1}^{-1} t .
$$

For simplicity, let us assume $K^{p}=\prod_{l \neq p} \mathrm{GL}_{2}\left(\mathbb{Z}_{l}\right)$. In general, we can use Hecke operators at ramified places as in Lemma 6.2.6. Now the key point is that $\tilde{H}^{1}\left(K^{p}, C\right)\left[\mathfrak{p}_{\lambda \cdot t}\right]$ contains a copy of $\rho \otimes \Pi(\rho(1))$. Hence, by Remark 6.4.5 (with $V(-1)=\rho$ here),

$$
\tilde{H}^{1}\left(K^{p}, C\right)_{(0,1)}^{\mathrm{la}}\left[\mathfrak{p}_{\lambda}\right]^{P_{0}, U_{p}=0}=\tilde{H}^{1}\left(K^{p}, C\right)^{\mathrm{la}}\left[\mathfrak{p}_{\lambda}\right]^{P_{0}, U_{p}=0}
$$

has dimension (over $C$ ) at least $\left(\operatorname{dim}_{\overline{\mathbb{Q}}_{p}} \rho\right) \times\left(\operatorname{dim}_{\overline{\mathbb{Q}}_{p}}(\rho \otimes C)^{G_{Q_{p}}}\right)=4$. But this contradicts the exact sequence above as $\operatorname{dim}_{C}\left(M_{1}^{\dagger}\left(K^{p}\right)\left[\mathfrak{p}_{\lambda}\right] \cdot e_{1}^{-1} t\right)^{P_{0}, U_{p}=0}=1$ by $q$-expansion principle and taking $P_{0^{-}}$ invariants and kernel of $U_{p}$ are left-exact. Hence, $\rho$ has to be classical.

\footnotetext{
Acknowledgement. I thank Matthew Emerton for useful discussions and suggesting the possibility of applying Colmez's Kirillov model to the problem of modularity of weight 1 forms. I thank Richard Taylor for a key conversation on possible structure in Theorem 1.0.2. I also thank Tsao-Hsien Chen, Pierre Colmez, Weibo Fu, Kai-Wen Lan, Ruochuan Liu, Jun Su and Xinwen Zhu for answering my questions and helpful discussions. Further, I thank Michael Harris and Kai-Wen Lan for several comments and corrections on an earlier version of this article. Finally, I am very grateful to the anonymous referees for their detailed comments on this article. I am supported by a Simons Junior Faculty Fellows award from the Simons Foundation.
}

Financial Support. I am supported by a Simons Junior Faculty Fellows award from the Simons Foundation. 


\section{References}

[AGT16] A. Abbes, M. Gros and T. Tsuji, The p-Adic Simpson Correspondence, Annals of Mathematics Studies, Vol. 193, (Princeton University Press, Princeton, NJ, 2016). MR 3444777.

[AIS14] F. Andreatta, A. Iovita and G. Stevens, 'Overconvergent modular sheaves and modular forms for GL $2 / \mathrm{F}$ ', Israel J. Math. 201(1) (2014), 299-359. MR 3265287.

[Ard17] K. Ardakov, 'Equivariant $\mathcal{D}$-modules on rigid analytic spaces', Astérisque (2017), to appear.

[Beř84] A. Beřlinson, 'Localization of representations of reductive Lie algebras', in Proceedings of the International Congress of Mathematicians, Vol. 1, 2 (Warsaw, 1983) (PWN, Warsaw, 1984), 699-710. MR 804725.

[BB83] A. Berlinson and J. Bernstein, 'A generalization of Casselman's submodule theorem', in Representation Theory of Reductive Groups (Park City, Utah, 1982), Progr. Math., Vol. 40 (Birkhäuser Boston, Boston, 1983), 35-52. MR 733805.

[BB10] L. Berger and C. Breuil, 'Sur quelques représentations potentiellement cristallines de $\mathrm{GL}_{2}\left(\mathbf{Q}_{\mathrm{p}}\right)$ ', Astérisque 330 (2010), 155-211. MR 2642406.

[BC08] L. Berger and P. Colmez, 'Familles de représentations de de Rham et monodromie p-adique', Astérisque 319 (2008), 303-337. MR 2493221.

[BC16] L. Berger and P. Colmez, 'Théorie de Sen et vecteurs localement analytiques', Ann. Sci. Éc. Norm. Supér. (4) 49(4) (2016), 947-970. MR 3552018.

[BW00] A. Borel and N. Wallach, Continuous Cohomology, Discrete Subgroups, and Representations of Reductive Groups, second ed., Mathematical Surveys and Monographs, Vol. 67 (American Mathematical Society, Providence, RI, 2000). MR 1721403.

[BLR91] N. Boston, H. W. Lenstra, Jr. and K. A. Ribet, Quotients of group rings arising from two-dimensional representations', 'C. R. Acad. Sci. Paris Sér. I Math. 312(4) (1991), 323-328. MR 1094193.

[Bou60] N. Bourbaki, Éléments de mathématique. XXVI. Groupes et algèbres de Lie. Chapitre 1: Algèbres de Lie, Actualités Sci. Ind. No. 1285 (Hermann, Paris, 1960). MR 0132805.

[BP21] G. Boxer and V. Pilloni, Higher Coleman theory, Preprint, 2021, URL: https://perso.ens-lyon.fr/ vincent.pilloni/HigherColeman.pdf.

[BE10] C. Breuil and M. Emerton, 'Représentations p-adiques ordinaires de $\mathrm{GL}_{2}\left(\mathbf{Q}_{\mathrm{p}}\right)$ et compatibilité local-global', Astérisque 331 (2010), 255-315. MR 2667890.

[BT99] K. Buzzard and R. Taylor, 'Companion forms and weight one forms', Ann. Math. (2) 149(3) (1999), 905-919. MR 1709306.

[CE12] F. Calegari and M. Emerton, 'Completed cohomology—a survey', in Non-Abelian Fundamental Groups and Iwasawa Theory, London Math. Soc. Lecture Note Ser., Vol. 393 (Cambridge Univ. Press, Cambridge, 2012), $239-257$. MR 2905536.

[CG18] F. Calegari and D. Geraghty, 'Modularity lifting beyond the Taylor-Wiles method', Invent. Math. 211(1) (2018), 297-433. MR 3742760.

[Cam21] J. E. Rodríguez Camargo, Eichler-Shimura maps for the modular curve, Preprint, 2021, URL: https://arxiv.org/abs/ 2102.13099.

[CS17] A. Caraiani and P. Scholze, 'On the generic part of the cohomology of compact unitary Shimura varieties', Ann. Math. (2) 186(3) (2017), 649-766. MR 3702677.

[Che14] G. Chenevier, 'The $p$-adic analytic space of pseudocharacters of a profinite group and pseudorepresentations over arbitrary rings', in Automorphic Forms and Galois Representations. Vol. 1, London Math. Soc. Lecture Note Ser., vol. 414 (Cambridge Univ. Press, Cambridge, 2014), 221-285. MR 3444227.

[CHJ17] P. Chojecki, D. Hansen and C. Johansson, 'Overconvergent modular forms and perfectoid Shimura curves', Doc. Math. 22 (2017), 191-262. MR 3609197.

[Cla66] D. N. Clark, 'A note on the $p$-adic convergence of solutions of linear differential equations', Proc. Amer. Math. Soc. 17 (1966), 262-269. MR 186895.

[CM98] R. Coleman and B. Mazur, 'The eigencurve', in Galois Representations in Arithmetic Algebraic Geometry (Durham, 1996), London Math. Soc. Lecture Note Ser., Vol. 254 (Cambridge Univ. Press, Cambridge, 1998), 1-113. MR 1696469.

[Col10] P. Colmez, 'Représentations de $G L_{2}\left(\boldsymbol{Q}_{p}\right)$ et ( $\phi, \Gamma$ )-modules', Astérisque 330 (2010), 281-509. MR 2642409.

[CD14] P. Colmez and G. Dospinescu, 'Complétés universels de représentations de $\mathrm{GL}_{2}\left(\mathbb{Q}_{p}\right)$ ', Algebra Number Theory 8(6) (2014), 1447-1519. MR 3267142.

[CDP14] P. Colmez, G. Dospinescu and V. Paškūnas, 'The $p$-adic local Langlands correspondence for $G L_{2}\left(\mathbb{Q}_{p}\right)$ ', Camb. J. Math. 2(1) (2014), 1-47. MR 3272011.

[De171] P. Deligne, 'Formes modulaires et représentations l-adiques', in Séminaire Bourbaki. Vol. 1968/69: Exposés 347-363, Lecture Notes in Math., Vol. 175 (Springer, Berlin, 1971), 139-172. MR 3077124.

[DS74] P. Deligne and J.-P. Serre, 'Formes modulaires de poids 1', Ann. Sci. École Norm. Sup. (4) 7 (1974), 507-530. MR 379379.

[DLLZ18] H. Diao, K.-W. Lan, R. Liu and X. Zhu, Logarithmic Riemann-Hilbert Correspondences for Rigid Varieties, Preprint, 2018, URL: https://www-users.cse.umn.edu/ kwlan/articles/log-RH.pdf. 
[DLLZ19] H. Diao, K.-W. Lan, R. Liu and X. Zhu, Logarithmic Adic Spaces: Some Foundational Results, 2019, Preprint, URL: https://www-users.cse.umn.edu/ kwlan/articles/log-adic.pdf.

[Dos12] G. Dospinescu, 'Actions infinitésimales dans la correspondance de Langlands locale p-adique', Math. Ann. 354(2) (2012), 627-657. MR 2965255.

[DLB17] G. Dospinescu and A.-C. Le Bras, 'Revêtements du demi-plan de Drinfeld et correspondance de Langlands $p$-adique', Ann. Math. (2) 186(2) (2017), 321-411. MR 3702670.

[DPS20] G. Dospinescu, V. Paškūnas and B. Schraen, Infinitesimal Characters in Arithmetic Families, Preprint, 2020, URL: https://arxiv.org/abs/2012.01041.

[DS13] G. Dospinescu and B. Schraen, 'Endomorphism algebras of admissible $p$-adic representations of $p$-adic Lie groups', Represent. Theory 17 (2013), 237-246. MR 3053464.

[Elk90] R. Elkik, 'Solutions d'équations à coefficients dans un anneau hensélien', Ann. Sci. École Norm. Sup. (4) 6 (1973), 553-603, MR 345966.

[Eme06a] M. Emerton, 'A local-global compatibility conjecture in the $p$-adic Langlands programme for $G L_{2 / Q}$ ', Pure Appl. Math. Q. 2(2) (2006), 279-393. Special Issue: In honor of John H. Coates. Part 2. MR 2251474.

[Eme06b] M. Emerton, 'On the interpolation of systems of eigenvalues attached to automorphic Hecke eigenforms', Invent. Math. 164(1) (2006), 1-84. MR 2207783.

[Eme11] M. Emerton, 'Local-global compatibility in the p-adic Langlands programme for $G L_{2} / Q$ ', Preprint, 2011, URL: http://www.math.uchicago.edu/ emerton/pdffiles/lg.pdf.

[EPW] M. Emerton, R. Pollack and T. Weston, 'Explicit reciprocity laws and Iwasawa theory for modular forms', in preparation.

[Fal87] G. Faltings, 'Hodge-Tate structures and modular forms', Math. Ann. 278(1-4) (1987), 133-149. MR 909221.

[Fal05] G. Faltings, 'A p-adic Simpson correspondence', Adv. Math. 198(2) (2005), 847-862. MR 2183394.

[Fon04] J.-M. Fontaine, 'Arithmétique des représentations galoisiennes p-adiques', Astérisque 295 (2004), xi, 1-115. MR 2104360.

[Gou94] F. Q. Gouvêa, 'Continuity properties of $p$-adic modular forms', in Elliptic Curves and Related Topics, CRM Proc. Lecture Notes, Vol. 4 (Amer. Math. Soc., Providence, RI, 1994), 85-99. MR 1260956.

[Gro57] A. Grothendieck, 'Sur quelques points d'algèbre homologique', Tohoku Math. J. 9(2) (1957), 119-221. MR 102537.

[Han16] D. Hansen, Quotients of Adic Spaces by Finite Groups, Math. Res. Letters (2016), to appear.

[Hid89] H. Hida, Nearly ordinary Hecke algebras and Galois representations of several variables, in Algebraic Analysis, Geometry, and Number Theory (Baltimore, MD, 1988) (Johns Hopkins Univ. Press, Baltimore, MD, 1989), $115-134$. MR 1463699.

[How21] S. Howe, 'Overconvergent modular forms are highest-weight vectors in the Hodge-Tate weight zero part of completed cohomology', Forum Math. Sigma 9 (2021), e17.

[Hub96] R. Huber, Étale Cohomology of Rigid Analytic Varieties and Adic Spaces, Aspects of Mathematics, Vol. E30 (Friedr. Vieweg \& Sohn, Braunschweig, 1996). MR 1734903.

[KS94] M. Kashiwara and W. Schmid, 'Quasi-equivariant $D$-modules, equivariant derived category, and representations of reductive Lie groups', in Lie Theory and Geometry, Progr. Math., Vol. 123 (Birkhäuser Boston, Boston, 1994), 457488. MR 1327544.

[Kat73] N. M.Katz, 'p-Adic properties of modular schemes and modular forms', in Modular Functions of One Variable, Vol. III (Proc. Internat. Summer School, Univ. Antwerp, Antwerp, 1972), Lecture Notes in Mathematics, Vol. 350 (Springer-Verlag, Berlin, New York, 1973), 69-190. MR 0447119.

[KM85] N. M. Katz and B. Mazur, Arithmetic Moduli of Elliptic Curves, Annals of Mathematics Studies, Vol. 108 (Princeton University Press, Princeton, NJ, 1985). MR 772569.

[LZ17] R. Liu and X. Zhu, 'Rigidity and a Riemann-Hilbert correspondence for $p$-adic local systems', Invent. Math. 207(1) (2017), 291-343. MR 3592758.

[Mum77] D. Mumford, 'Hirzebruch's proportionality theorem in the noncompact case', Invent. Math. 42 (1977), 239-272. MR 471627.

[Pan19] L. Pan, The Fontaine-Mazur Conjecture in the Residually Reducible Case, J. Amer. Math. Soc. (2019), to appear.

[Pan20] L. Pan, A Note on Overconvergence of Hecke Action, Preprint, 2020, arxiv:2012.11845.

[Paš13] V. Paškūnas, 'The image of Colmez's Montreal functor', Publ. Math. Inst. Hautes Études Sci. 118 (2013), 1-191. MR 3150248.

[Paš16] V. Paškūnas, 'On 2-dimensional 2-adic Galois representations of local and global fields', Algebra Number Theory 10(6) (2016), 1301-1358. MR 3544298.

[Pas18] V. Paskunas, 'On some consequences of a theorem of J. Ludwig', Preprint, 2018, arxiv:1804.07567.

[PT21] V. Paškūnas and S.-N. Tung, 'Finiteness properties of the category of mod prepresentations of $G L_{2}\left(\mathbb{Q}_{p}\right)$ ', Forum Math. Sigma. 9 (2021), Paper No. e80, 39. MR 4350140.

[Pil13] V. Pilloni, 'Overconvergent modular forms', Ann. Inst. Fourier (Grenoble) 63(1) (2013), 219-239. MR 3097946.

[PS16] V. Pilloni and B. Stroh, 'Surconvergence, ramification et modularité', Astérisque 382 (2016), 195-266. MR 3581178.

[Sch11] P. Schneider, p -Adic Lie Groups, Grundlehren der Mathematischen Wissenschaften [Fundamental Principles of Mathematical Sciences], Vol. 344 (Springer, Heidelberg, 2011). MR 2810332. 
[ST02] P. Schneider and J. Teitelbaum, 'Locally analytic distributions and $p$-adic representation theory, with applications to GL2', J. Amer. Math. Soc. 15(2) (2002), 443-468. MR 1887640.

[ST03] P. Schneider and J. Teitelbaum, 'Algebras of $p$-adic distributions and admissible representations', Invent. Math. 153(1) (2003), 145-196. MR 1990669.

[Sch12] P. Scholze, 'Perfectoid spaces', Publ. Math. Inst. Hautes Études Sci. 116 (2012), 245-313. MR 3090258.

[Sch13b] P. Scholze, 'Perfectoid spaces: a survey', in Current Developments in Mathematics 2012 (Int. Press, Somerville, MA, 2013), 193-227. MR 3204346.

[Sch13a] P. Scholze, 'p-Adic Hodge theory for rigid-analytic varieties', Forum Math. Pi 1 (2013), e1, 77. MR 3090230.

[Sch15] P. Scholze, 'On torsion in the cohomology of locally symmetric varieties', Ann. Math. (2) 182(3) (2015), 945-1066. MR 3418533.

[Sch16] P. Scholze, ' $p$-Adic Hodge theory for rigid-analytic varieties—corrigendum' [MR3090230], Forum Math. Pi 4 (2016), e6, 4. MR 3535697.

[Sen81] S. Sen, 'Continuous cohomology and p-adic Galois representations', Invent. Math. 62(1) (1980/81), 89-116. MR 595584.

[Sta20] The Stacks Project authors, The stacks project, https://stacks.math.columbia.edu, 2020.

[Wei77] J. Weisinger, Some Results on Classical Eisenstein Series and Modular Forms over Function Fields (ProQuest LLC, Ann Arbor, MI, 1977). MR 2940742. 